\title{
Numerical methods for nonlocal and fractional models
}

\author{
Marta D'Elia ${ }^{1}$, Qiang Du ${ }^{2}$, Christian Glusa ${ }^{1}$, \\ Max Gunzburger ${ }^{3}$, Xiaochuan Tian ${ }^{4}$ and Zhi Zhou ${ }^{5}$ \\ 1 Center for Computing Research, \\ Sandia National Laboratories, \\ Albuquerque, New Mexico 87185, USA \\ E-mail: mdelia@sandia.gov, caglusa@sandia.gov \\ https://sites.google.com/site/martadeliawebsite \\ 2 Department of Applied Physics and Applied Mathematics \\ and Data Science Institute, \\ Columbia University, \\ New York, NY 10027, USA \\ E-mail: qd2125@columbia.edu \\ http://www.columbia.edu/ qd2125 \\ ${ }^{3}$ Department of Scientific Computing, \\ Florida State University, \\ Tallahassee, Florida 32306, USA \\ E-mail: mgunzburger@fsu.edu \\ https://www.sc.fsu.edu/gunzburg \\ 4 Department of Mathematics, \\ University of Texas, \\ Austin, Texas 78751, USA \\ E-mail: xtian@math.utexas.edu \\ https://web.ma.utexas.edu/users/xtian \\ Department of Applied Mathematics, \\ The Hong Kong Polytechnic University, \\ Kowloon, Hong Kong, China \\ E-mail: zhizhou@polyu.edu.hk \\ https://sites.google.com/site/zhizhou0125
}


Partial differential equations (PDEs) are used with huge success to model phenomena across all scientific and engineering disciplines. However, across an equally wide swath, there exist situations in which PDEs fail to adequately model observed phenomena, or are not the best available model for that purpose. On the other hand, in many situations, nonlocal models that account for interaction occurring at a distance have been shown to more faithfully and effectively model observed phenomena that involve possible singularities and other anomalies. In this article we consider a generic nonlocal model, beginning with a short review of its definition, the properties of its solution, its mathematical analysis and of specific concrete examples. We then provide extensive discussions about numerical methods, including finite element, finite difference and spectral methods, for determining approximate solutions of the nonlocal models considered. In that discussion, we pay particular attention to a special class of nonlocal models that are the most widely studied in the literature, namely those involving fractional derivatives. The article ends with brief considerations of several modelling and algorithmic extensions, which serve to show the wide applicability of nonlocal modelling.

\section{CONTENTS}

PART 1: Nonlocal diffusion models, including fractional models

1 General models for nonlocal diffusion

2 Weak formulations of nonlocal models

3 Fractional diffusion models

PART 2: Numerical methods for nonlocal and fractional models

4 Introductory remarks

5 Finite element methods for nonlocal models

6 Finite element methods for the integral fractional Laplacian

7 Finite element methods for the spectral fractional Laplacian

8 Spectral-Galerkin methods for nonlocal diffusion

9 Spectral-Galerkin methods for fractional diffusion

10 Finite difference methods for the strong form of nonlocal diffusion

11 Numerical methods for the strong form of fractional diffusion

12 Conditioning and fast solvers 
PART 3: Selected extensions

13 Weakly coercive, indefinite and non-self-adjoint problems

14 Nonlocal convection-diffusion problems $\quad 77$

15 Time-dependent nonlocal problems $\quad 82$

16 Inverse problems $\quad 85$

17 Variational inequalities and obstacle problems $\quad 93$

18 Reduced-order modelling

19 A turbulent flow application

20 Peridynamics models for solid mechanics

21 Image denoising

References

\section{PART ONE}

\section{Nonlocal diffusion models, including fractional models}

In order to make the article as self-contained as possible, in this part we introduce the model equations and discuss solutions of those equations used in the development and analysis of the numerical methods described in Part 2. We focus on a general class of nonlocal models that are characterized by interactions at a distance via integral equation formulations as opposed to partial differential equations. For the same applications, the nonlocal models considered provide different representations of physics ${ }^{1}$ compared to partial differential equation models. Fractional derivative models are an example of the general class we consider. Because there exists a large mathematical and computational literature devoted to fractional derivative models, that class is given special attention throughout the article. However, also highlighted are the opportunities afforded by the more general nonlocal models we consider that are not available through the use of fractional models.

We use the following notational conventions throughout. Exceptions should not cause confusion. Please note that the adherence to these conventions becomes less strict as one moves from Part 1 to Part 2 to Part 3.

1 Throughout, for the sake of economy of exposition, we refer to physics as the setting for the generic models we consider. Of course, those models also arise in all other physical science settings, as well as in engineering and the biological and social sciences. 
Roman letters

Greek letters

plain font

boldface font

upper-case boldface font calligraphy font functions depending on a single point, e.g. $u(\boldsymbol{x}), \boldsymbol{v}(\boldsymbol{x}), \boldsymbol{D}(\boldsymbol{x})$

functions depending on two points, e.g. $\eta(\boldsymbol{x}, \boldsymbol{y}), \boldsymbol{\nu}(\boldsymbol{x}, \boldsymbol{y}), \boldsymbol{\Theta}(\boldsymbol{x}, \boldsymbol{y})$ scalar-valued functions, e.g. $u(\boldsymbol{x}), \eta(\boldsymbol{x}, \boldsymbol{y})$ vector-valued functions, e.g. $\boldsymbol{u}(\boldsymbol{x}), \boldsymbol{\nu}(\boldsymbol{x}, \boldsymbol{y})$ tensor-valued functions, e.g. $\boldsymbol{D}(\boldsymbol{x}), \boldsymbol{\Theta}(\boldsymbol{x}, \boldsymbol{y})$ operators, functionals, bilinear forms, e.g. $\mathcal{A}, \mathcal{D}, \mathcal{L}$

In choosing these notational conventions, our goal is to be as consistent as possible or practical throughout the article. Unfortunately, because of the different notations adopted in the literature, the notations used in the article may differ from those used in some of the cited books and papers.

\section{General models for nonlocal diffusion}

We consider an integral equation model that is a nonlocal analogue of the classical Poisson problem

$$
\begin{cases}-\mathcal{L}_{0} u:=-\nabla \cdot(D \nabla u)=f(\boldsymbol{x}) & \text { for all } \boldsymbol{x} \in \Omega \\ \mathcal{B} u=g(\boldsymbol{x}) & \text { for all } \boldsymbol{x} \in \partial \Omega\end{cases}
$$

where $\Omega \in \mathbb{R}^{d}$ denotes a bounded, open domain having boundary $\partial \Omega, f(\boldsymbol{x})$ and $g(\boldsymbol{x})$ denote given functions defined on $\Omega$ and $\partial \Omega$, respectively, and $\boldsymbol{D}(\boldsymbol{x})$ denotes a given symmetric, positive definite $d \times d$ matrix. For the boundary conditions operator $\mathcal{B}$, we have the choices

$$
\mathcal{B} u= \begin{cases}u & \Leftarrow \text { Dirichlet boundary condition, } \\ (\boldsymbol{D} \nabla u) \cdot \boldsymbol{n} & \Leftarrow \text { Neumann boundary condition, } \\ (\boldsymbol{D} \nabla u) \cdot \boldsymbol{n}+r(\boldsymbol{x}) u & \Leftarrow \text { Robin boundary condition, }\end{cases}
$$

where $r(\boldsymbol{x})$ denotes a given function, as well as mixed boundary conditions, for example Dirichlet and Neumann boundary conditions applied on disjoint, covering parts of the boundary. Problem (1.1) is a model for steady-state diffusion, for example.

As will be immediately clear, the nonlocal model we consider is not an integral or boundary integral reformulation of problem (1.1) based on, for example, the use of Green's functions, but rather it models different physics from (1.1). 


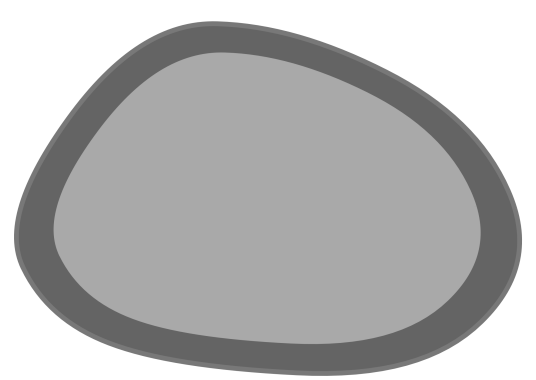

(a)

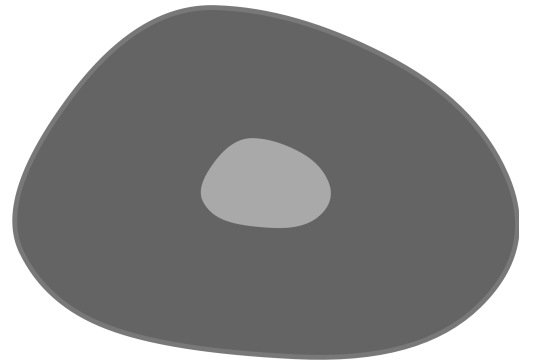

(b)

Figure 1.1. $\Omega$ is light grey whereas $\Omega_{\mathcal{I}_{\delta}}$ is dark grey and has thickness $\delta$. (a) $\delta$ smaller than the diameter of $\Omega$, (b) $\delta$ larger than the diameter of $\Omega$.

Given the bounded, open domain $\Omega \in \mathbb{R}^{d}$ and given a constant $\delta>0$, we define the interaction domain corresponding to $\Omega$ as

$$
\Omega_{\mathcal{I}_{\delta}}:=\left\{\boldsymbol{y} \in \mathbb{R}^{d} \backslash \Omega \text { such that } \boldsymbol{y} \in B_{\delta}(\boldsymbol{x}) \text { for some } \boldsymbol{x} \in \Omega\right\},
$$

where $B_{\delta}(\boldsymbol{x})$ denotes the ball of radius $\delta$ centred at $\boldsymbol{x}$. We refer to $\delta$ as the interaction radius or horizon. The nomenclature 'interaction' used here is appropriate because $\Omega_{\mathcal{I}_{\delta}}$ contains all points in the complement domain $\mathbb{R}^{d} \backslash \Omega$ that are within a distance $\delta$ of some point in $\Omega$. Note that the four cases

$$
0<\delta \ll \operatorname{diam} \Omega, \quad \operatorname{diam} \Omega \ll \delta<\infty, \quad \delta \approx \operatorname{diam} \Omega, \quad \delta=\infty
$$

are all of interest. An illustration of the first two cases is given in Figure 1.1. For the fourth case we have that the interaction domain $\Omega_{\mathcal{I}_{\infty}}=\mathbb{R}^{d} \backslash \Omega$. Also, the case of $\Omega=\mathbb{R}^{d}$ is of considerable interest.

For $\delta>0$, we consider the nonlocal problem for a scalar-valued function $u(\boldsymbol{x})$ defined on $\Omega \cup \Omega_{\mathcal{I}_{\delta}}$, given by

$$
\begin{cases}-\mathcal{L}_{\delta} u=f(\boldsymbol{x}) & \text { for all } \boldsymbol{x} \in \Omega \\ \mathcal{V} u=g(\boldsymbol{x}) & \text { for all } \boldsymbol{x} \in \Omega_{\mathcal{I}_{\delta}} .\end{cases}
$$

Note that the constraint $\mathcal{V} u=g$ is applied on the domain $\Omega_{\mathcal{I}_{\delta}}$ having nonzero volume in $\mathbb{R}^{d}$, in contrast to the constraint $\mathcal{B} u=g$ in (1.1), which is applied on the boundary surface $\partial \Omega$. For this reason we refer to $\mathcal{V} u=g$ as being a volume constraint and to (1.5) as a being a volume-constrained problem (Du, Gunzburger, Lehoucq and Zhou 2012a).

In (1.5), we have that $f(\boldsymbol{x})$ and $g(\boldsymbol{x})$ denote given scalar-valued functions defined on $\Omega$ and $\Omega_{\mathcal{I}_{\delta}}$, respectively, and

$$
\mathcal{L}_{\delta} u(\boldsymbol{x}):=2 \int_{\Omega \cup \Omega_{\mathcal{I}_{\delta}}}(u(\boldsymbol{y})-u(\boldsymbol{x})) \gamma_{\delta}(\boldsymbol{x}, \boldsymbol{y}) \mathrm{d} \boldsymbol{y} \quad \text { for all } \boldsymbol{x} \in \Omega,
$$


where $\gamma_{\delta}(\boldsymbol{x}, \boldsymbol{y})$ is a symmetric function, that is,

$$
\gamma_{\delta}(\boldsymbol{x}, \boldsymbol{y})=\gamma_{\delta}(\boldsymbol{y}, \boldsymbol{x})
$$

and, for any $\boldsymbol{x}$,

$$
\operatorname{supp}\left(\gamma_{\delta}(\boldsymbol{x}, \boldsymbol{y})\right)=B_{\delta}(\boldsymbol{x})
$$

that is, $\gamma_{\delta}(\boldsymbol{x}, \boldsymbol{y})=0$ whenever $|\boldsymbol{y}-\boldsymbol{x}|>\delta$. We note that in some of the specific cases we consider below, the integral in (1.6) has to be viewed in the principal value sense. For notational simplicity, we omit 'p.v.' in front of the integral sign even in such cases. The operator $\mathcal{L}_{\delta}$ is a nonlocal analogue of the partial differential equation (PDE) operator $\nabla \cdot(D \nabla u)$. That connection is made explicit in Section 1.1. The nonlocal model (1.5) is a nonlocal analogue of the local PDE diffusion model (1.1) model.

Analogous to (1.2), we have the choice of volume constraints

$$
\mathcal{V} u= \begin{cases}u & \Leftarrow \text { Dirichlet volume constraint } \\ \mathcal{N}_{\delta} u & \Leftarrow \text { Neumann volume constraint } \\ \mathcal{N}_{\delta} u+r(\boldsymbol{x}) u & \Leftarrow \text { Robin volume constraint }\end{cases}
$$

as well as mixed types of volume constraints, where the linear operator $\mathcal{N}_{\delta}$ can be defined in several ways, for example,

$$
\mathcal{N}_{\delta} u:=-2 \int_{\Omega \cup \Omega_{\mathcal{I}_{\delta}}}(u(\boldsymbol{y})-u(\boldsymbol{x})) \gamma_{\delta}(\boldsymbol{x}, \boldsymbol{y}) \mathrm{d} \boldsymbol{y} \quad \text { for all } \boldsymbol{x} \in \Omega_{\mathcal{I}_{\delta}} .
$$

It is obvious that the definitions of the operators $\mathcal{L}_{\delta}$ and $\mathcal{N}_{\delta}$ involve exactly the same integrand but have different domains of definition, i.e. $\Omega$ for the former and $\Omega_{\mathcal{I}_{\delta}}$ for the latter. So, clearly, they can be combined into a single operator defined over $\Omega \cup \Omega_{\mathcal{I}_{\delta}}$. However, it is convenient to keep using the two operators.

Because points $\boldsymbol{x}$ only interact with points $\boldsymbol{y} \in B_{\delta}(\boldsymbol{x}),(1.6)$ and (1.10) can be equivalently written as

$$
\mathcal{L}_{\delta} u:=2 \int_{B_{\delta}(\boldsymbol{x})}(u(\boldsymbol{y})-u(\boldsymbol{x})) \gamma_{\delta}(\boldsymbol{x}, \boldsymbol{y}) \mathrm{d} \boldsymbol{y} \quad \text { for all } \boldsymbol{x} \in \Omega
$$

and

$$
\mathcal{N}_{\delta} u:=-2 \int_{\left(\Omega \cup \Omega_{\mathcal{I}_{\delta}}\right) \cap B_{\delta}(\boldsymbol{x})}(u(\boldsymbol{y})-u(\boldsymbol{x})) \gamma_{\delta}(\boldsymbol{x}, \boldsymbol{y}) \mathrm{d} \boldsymbol{y} \quad \text { for all } \boldsymbol{x} \in \Omega_{\mathcal{I}_{\delta}},
$$

respectively, where we have used the facts that due to the definition of the interaction domain $\Omega_{\mathcal{I}_{\delta}},\left(\Omega \cup \Omega_{\mathcal{I}_{\delta}}\right) \cap B_{\delta}(\boldsymbol{x})=B_{\delta}(\boldsymbol{x})$ for $\boldsymbol{x} \in \Omega$ but $\left(\Omega \cup \Omega_{\mathcal{I}_{\delta}}\right) \cap B_{\delta}(\boldsymbol{x}) \subset B_{\delta}(\boldsymbol{x})$ for $\boldsymbol{x} \in \Omega_{\mathcal{I}_{\delta}}$. 


\subsection{A brief review of a nonlocal vector calculus}

To make some sense of how the model (1.5) arises and why we refer to it as a nonlocal analogue of the model (1.1), we need to first introduce some elements of a nonlocal vector calculus. Note that in this article we also use elements of the fractional calculus. However, because there are many excellent references about the fractional calculus and its applications (see e.g. Baleanu, Diethelm, Scalas and Trujillo 2016, Mainardi 1997, Meerschaert and Sikorskii 2012), we do not discuss them here.

The classical vector calculus provides a set of tools that are in ubiquitous use for the modelling, analysis, discretization and numerical analysis of PDE models, an obvious example being the use of Green's first identity to transform the strong formulation of a PDE into a weak formulation. The foundations of that calculus are the familiar divergence, gradient and curl differential operators, upon which an edifice is built that includes vector identities (e.g. div curl $\boldsymbol{v}=0$ ), integral theorems (e.g. Gauss's theorem) and much, much more.

A nonlocal vector calculus has been developed (Gunzburger and Lehoucq 2010, Du et al. 2012a, Du, Gunzburger, Lehoucq and Zhou 2013a, Alali, Gunzburger and Liu 2015, Mengesha and Du 2016, Du 2019, D'Elia et al. 2019 a) to deal with nonlocal models such as (1.5) in much the same way as the classical vector calculus is used to deal with PDE models such as (1.1). Here, mostly following Du et al. (2013a), we provide a brief introduction to the nonlocal vector calculus, including notions that are used in the rest of the article. We remark that certain elements of the nonlocal vector calculus have previously appeared in Coulhon and Grigoryan (1998), Zhou and Schölkopf (2005), Gilboa and Osher (2008), Lézoray, Ta and Elmoataz (2010), Lou, Zhang, Osher and Bertozzi (2010) and Jiang, Lim, Yao and Ye (2011), for example. However, the discussions in those papers, compared to that in Du et al. $(2013 a)$, are limited in scope and in application and provide only a partial development of a nonlocal vector calculus that mimics the classical vector calculus.

The foundation of the nonlocal vector calculus is integral operators that mimic the three differential operators upon which the classical vector calculus is built.

Given the vector-valued functions $\boldsymbol{\nu}_{\delta}(\boldsymbol{x}, \boldsymbol{y}):\left(\Omega \cup \Omega_{\mathcal{I}_{\delta}}\right) \times\left(\Omega \cup \Omega_{\mathcal{I}_{\delta}}\right) \rightarrow \mathbb{R}^{d}$ and $\boldsymbol{\alpha}_{\delta}(\boldsymbol{x}, \boldsymbol{y}):\left(\Omega \cup \Omega_{\mathcal{I}_{\delta}}\right) \times\left(\Omega \cup \Omega_{\mathcal{I}_{\delta}}\right) \rightarrow \mathbb{R}^{d}$, the action of the nonlocal $d i$ vergence operator $\mathcal{D}_{\delta}: \Omega \cup \Omega_{\mathcal{I}_{\delta}} \rightarrow \mathbb{R}$ on $\boldsymbol{\nu}(\boldsymbol{x}, \boldsymbol{y})$ is defined as

$$
\left(\mathcal{D}_{\delta} \boldsymbol{\nu}\right)(\boldsymbol{x}):=\int_{\Omega \cup \Omega_{\mathcal{I}_{\delta}}}(\boldsymbol{\nu}(\boldsymbol{y}, \boldsymbol{x})+\boldsymbol{\nu}(\boldsymbol{x}, \boldsymbol{y})) \cdot \boldsymbol{\alpha}_{\delta}(\boldsymbol{x}, \boldsymbol{y}) \mathcal{X}_{B_{\delta}(\boldsymbol{x})}(\boldsymbol{y}) \mathrm{d} \boldsymbol{y}
$$

for all $\boldsymbol{x} \in \Omega \cup \Omega_{\mathcal{I}_{\delta}}$, where $\boldsymbol{\alpha}_{\delta}(\boldsymbol{x}, \boldsymbol{y})$ denotes an antisymmetric function, that is, $\boldsymbol{\alpha}_{\delta}(\boldsymbol{y}, \boldsymbol{x})=-\boldsymbol{\alpha}_{\delta}(\boldsymbol{x}, \boldsymbol{y})$ for all $\boldsymbol{x}, \boldsymbol{y} \in \Omega \cup \Omega_{\mathcal{I}_{\delta}}$, and $\mathcal{X}_{(\cdot)}(\cdot)$ denotes 
the indicator function. Note that unlike its differential counterpart $\nabla \cdot$, the nonlocal operator $\mathcal{D}_{\delta}$ is not uniquely defined, that is, not only do we have an unspecified parameter $\delta>0$ but also we have said nothing about $\boldsymbol{\alpha}_{\delta}(\boldsymbol{x}, \boldsymbol{y})$ other than it is an antisymmetric function. The choices one makes for $\delta$ and especially $\boldsymbol{\alpha}_{\delta}(\boldsymbol{x}, \boldsymbol{y})$ can result in operators having very different properties. Thus $\delta$ and $\boldsymbol{\alpha}_{\delta}(\boldsymbol{x}, \boldsymbol{y})$ are modelling choices dictated by the specific application one considers. We have already mentioned, in (1.4), the wide choices of $\delta$ that are of interest; several choices for $\boldsymbol{\alpha}_{\delta}(\boldsymbol{x}, \boldsymbol{y})$ are considered in Section 2.2.1.

Simple manipulations show that, under suitable regularity assumptions that we do not dwell on here,

$$
\int_{\Omega \cup \Omega_{\mathcal{I}_{\delta}}} v \mathcal{D}_{\delta} \boldsymbol{\nu} \mathrm{d} \boldsymbol{x}=\int_{\Omega \cup \Omega_{\mathcal{I}_{\delta}}} \int_{\Omega \cup \Omega_{\mathcal{I}_{\delta}}} \boldsymbol{\nu} \cdot \mathcal{D}_{\delta}^{*} v \mathrm{~d} \boldsymbol{y} \mathrm{d} \boldsymbol{x}
$$

where

$$
\left(\mathcal{D}_{\delta}^{*} u\right)(\boldsymbol{x}, \boldsymbol{y}):=-(u(\boldsymbol{y})-u(\boldsymbol{x})) \boldsymbol{\alpha}_{\delta}(\boldsymbol{x}, \boldsymbol{y}) \mathcal{X}_{B_{\delta}(\boldsymbol{x})}(\boldsymbol{y})
$$

for all $\boldsymbol{x}, \boldsymbol{y} \in \Omega \cup \Omega_{\mathcal{I}_{\delta}}$. From (1.14), it is natural to refer to operator $\mathcal{D}_{\delta}^{*}$ as being the adjoint operator corresponding to $\mathcal{D}_{\delta}$. With $\mathcal{D}_{\delta}^{*}$ being the adjoint of $\mathcal{D}_{\delta}$, one may formally refer to $-\mathcal{D}_{\delta}^{*}$ as being a nonlocal gradient operator.

Let $\boldsymbol{\Theta}_{\delta}(\boldsymbol{x}, \boldsymbol{y}):\left(\Omega \cup \Omega_{\mathcal{I}_{\delta}}\right) \times\left(\Omega \cup \Omega_{\mathcal{I}_{\delta}}\right) \rightarrow \mathbb{R}^{d \times d}$ denote a tensor-valued function that is symmetric in the function sense, i.e. $\boldsymbol{\Theta}_{\delta}(\boldsymbol{x}, \boldsymbol{y})=\boldsymbol{\Theta}_{\delta}(\boldsymbol{y}, \boldsymbol{x})$, and symmetric and non-negative definite in the matrix sense. Then, from the definitions of the nonlocal divergence and gradient operators, we have that

$$
\begin{aligned}
- & \mathcal{D}_{\delta}\left(\boldsymbol{\Theta}_{\delta} \mathcal{D}_{\delta}^{*} u\right) \\
& =2 \int_{\Omega \cup \Omega_{\mathcal{I}_{\delta}}}(u(\boldsymbol{y})-u(\boldsymbol{x})) \boldsymbol{\alpha}_{\delta}(\boldsymbol{x}, \boldsymbol{y}) \cdot\left(\boldsymbol{\Theta}_{\delta}(\boldsymbol{x}, \boldsymbol{y}) \boldsymbol{\alpha}_{\delta}(\boldsymbol{x}, \boldsymbol{y})\right) \mathcal{X}_{B_{\delta}(\boldsymbol{x})}(\boldsymbol{y}) \mathrm{d} \boldsymbol{y} .
\end{aligned}
$$

For all $\boldsymbol{x}, \boldsymbol{y} \in \Omega \cup \Omega_{\mathcal{I}_{\delta}}$, we define the kernel $\gamma_{\delta}(\boldsymbol{x}, \boldsymbol{y})$ as

$$
\gamma_{\delta}(\boldsymbol{x}, \boldsymbol{y}):=\boldsymbol{\alpha}_{\delta}(\boldsymbol{x}, \boldsymbol{y}) \cdot\left(\boldsymbol{\Theta}_{\delta}(\boldsymbol{x}, \boldsymbol{y}) \boldsymbol{\alpha}_{\delta}(\boldsymbol{x}, \boldsymbol{y})\right) \mathcal{X}_{B_{\delta}(\boldsymbol{x})}(\boldsymbol{y}) .
$$

Note that $\gamma_{\delta}(\boldsymbol{x}, \boldsymbol{y})$ defined in this way satisfies the symmetry condition (1.7) and the support condition (1.8), where the former follows from the fact that $\mathcal{X}_{B_{\delta}(\boldsymbol{x})}(\boldsymbol{y})$ is itself a symmetric function, that is, if $\boldsymbol{y} \in B_{\delta}(\boldsymbol{x})$ then necessarily $\boldsymbol{x} \in B_{\delta}(\boldsymbol{y})$.

We then have that (1.16) can be expressed as

$$
\begin{aligned}
\mathcal{D}_{\delta}\left(\boldsymbol{\Theta}_{\delta} \mathcal{D}_{\delta}^{*} u\right) & =-2 \int_{\Omega \cup \Omega_{\mathcal{I}_{\delta}}}(u(\boldsymbol{y})-u(\boldsymbol{x})) \gamma_{\delta}(\boldsymbol{x}, \boldsymbol{y}) \mathrm{d} \boldsymbol{y} \\
& =-2 \int_{B_{\delta}(\boldsymbol{x})}(u(\boldsymbol{y})-u(\boldsymbol{x})) \gamma_{\delta}(\boldsymbol{x}, \boldsymbol{y}) \mathrm{d} \boldsymbol{y},
\end{aligned}
$$


where $\gamma_{\delta}(\boldsymbol{x}, \boldsymbol{y})$ is given by (1.17) and $\mathcal{D}_{\delta}$ and $\mathcal{D}_{\delta}^{*}$ are given by (1.13) and (1.15), respectively.

Of course, we recognize that

$$
\mathcal{D}_{\delta}\left(\Theta_{\delta} \mathcal{D}_{\delta}^{*} u\right)=-\mathcal{L}_{\delta} u,
$$

where $\mathcal{L}_{\delta} u$ is defined in (1.6) or (1.11). The fact that the operator $\mathcal{L}_{\delta}$ can be written as a composition of nonlocal divergence and gradient operators justifies referring to (1.5) as a (variable coefficient) nonlocal Poisson problem, that is, $\mathcal{L}_{\delta} u$ may be viewed as a nonlocal analogue of $\nabla \cdot(\boldsymbol{D} \nabla u)$ for suitable choices of $\boldsymbol{\alpha}_{\delta}(\boldsymbol{x}, \boldsymbol{y})$, and, if $\boldsymbol{\Theta}_{\delta}$ is the identity tensor, $\mathcal{L}_{\delta} u$ is indeed a nonlocal analogue of the $\Delta u$.

Remark 1.1 (variable coefficients in nonlocal model). An advantage accruing from the nonlocal calculus is that, through compositions such as (1.16) or (1.18), it provides a natural means for generalizing nonlocal constant coefficient operators such as the fractional Laplace operator to variable coefficient settings in a manner completely analogous to how one defines variable coefficient operators in the PDE case. As an example, in Section 3.4, we describe one way in which this can be done for fractional diffusion models.

The final bit of the nonlocal vector calculus that we need is a (generalized) nonlocal Green's first identity. In the context of this section, that identity is given by (Du et al. 2013a)

$$
\int_{\Omega \cup \Omega_{\mathcal{I}_{\delta}}} v \mathcal{D}_{\delta}\left(\boldsymbol{\Theta}_{\delta} \mathcal{D}^{*} u\right) \mathrm{d} \boldsymbol{y}=\int_{\Omega \cup \Omega_{\mathcal{I}_{\delta}}} \int_{\Omega \cup \Omega_{\mathcal{I}_{\delta}}} \mathcal{D}_{\delta}^{*} v \cdot\left(\boldsymbol{\Theta}_{\delta} \mathcal{D}_{\delta}^{*}\right) u \mathrm{~d} \boldsymbol{y} \mathrm{d} \boldsymbol{x} .
$$

We have

$$
\mathcal{D}_{\delta}^{*} v \cdot\left(\boldsymbol{\Theta}_{\delta} \mathcal{D}_{\delta}^{*}\right) u=(v(\boldsymbol{y})-v(\boldsymbol{x}))(u(\boldsymbol{y})-u(\boldsymbol{x})) \gamma_{\delta}(\boldsymbol{x}, \boldsymbol{y}),
$$

so substituting $(1.18),(1.10)$ and $(1.21)$ into (1.20) results in the equivalent form of the nonlocal Green's first identity given by

$$
\begin{aligned}
& \int_{\Omega \cup \Omega_{\mathcal{I}_{\delta}}} \int_{\Omega \cup \Omega_{\mathcal{I}_{\delta}}}(v(\boldsymbol{y})-v(\boldsymbol{x}))(u(\boldsymbol{y})-u(\boldsymbol{x})) \gamma_{\delta}(\boldsymbol{x}, \boldsymbol{y}) \mathrm{d} \boldsymbol{y} \mathrm{d} \boldsymbol{x} \\
& =-\int_{\Omega} v(\boldsymbol{x}) \mathcal{L}_{\delta} u(\boldsymbol{x}) \mathrm{d} \boldsymbol{x}+\int_{\Omega_{\mathcal{I}_{\delta}}} v(\boldsymbol{x}) \mathcal{N}_{\delta} u(\boldsymbol{x}) \mathrm{d} \boldsymbol{x} .
\end{aligned}
$$

We recognize (1.22) as a nonlocal analogue of the (generalized) local Green's first identity

$$
\int_{\Omega} \nabla v \cdot(\boldsymbol{D} \nabla u) \mathrm{d} \boldsymbol{x}=-\int_{\Omega} v \nabla \cdot(\boldsymbol{D} \nabla u) \mathrm{d} \boldsymbol{x}+\int_{\partial \Omega} v \boldsymbol{n} \cdot(\boldsymbol{D} \nabla u) \mathrm{d} \boldsymbol{x} .
$$

Derivations in a more rigorous functional analytic setting of nonlocal integral 
identities similar to (1.22) and the connection to their local analogues can be found in Mengesha and Du (2016) and Du (2019).

Remark 1.2 (roles played in the kernel by operator definitions and constitutive functions). It is important to differentiate between the roles of the functions $\boldsymbol{\alpha}_{\delta}(\boldsymbol{x}, \boldsymbol{y})$ and $\boldsymbol{\Theta}_{\delta}(\boldsymbol{x}, \boldsymbol{y})$ in the nonlocal models we are considering. It is clear that $\boldsymbol{\alpha}_{\delta}(\boldsymbol{x}, \boldsymbol{y})$ serves to define operators (i.e. $\mathcal{D}_{\delta}, \mathcal{D}_{\delta}^{*}$ and $\mathcal{N}_{\delta}$ given by $(1.13),(1.15)$ and (1.10), respectively), irrespective of how those operators are used. On the other hand, $\boldsymbol{\Theta}_{\delta}(\boldsymbol{x}, \boldsymbol{y})$ serves as a constitutive function. Thus both $\boldsymbol{\alpha}_{\delta}(\boldsymbol{x}, \boldsymbol{y})$ and $\boldsymbol{\Theta}_{\delta}(\boldsymbol{x}, \boldsymbol{y})$ are modelling choices. Both influence the properties of solutions of nonlocal models such as their regularity. Of course, the situation is much the same in the local PDE case, that is, the operators $\nabla \cdot$ and $\nabla$ are defined irrespective of how they are used and $\boldsymbol{D}(\boldsymbol{x})$ denotes a constitutive tensor.

Remark 1.3 (the choices that define a nonlocal diffusion model). Recapitulating, to define a specific nonlocal diffusion model, one must make three modelling choices:

- the horizon $\delta$ that defines the extent of nonlocal interactions,

- the antisymmetric function $\boldsymbol{\alpha}_{\delta}(\boldsymbol{x}, \boldsymbol{y})$ that defines the nonlocal divergence operator $\mathcal{D}_{\delta}$ and nonlocal gradient operator $-\mathcal{D}_{\delta}^{*}$,

- the constitutive tensor $\boldsymbol{\Theta}_{\delta}(\boldsymbol{x}, \boldsymbol{y})$ that defines the properties of the media.

These three choices are all that enter into the definition (1.17) of the kernel $\gamma_{\delta}(\boldsymbol{x}, \boldsymbol{y})$, so specifying them uniquely defines the operator $\mathcal{L}_{\delta}$.

Remark 1.4 (additional operators of the nonlocal vector calculus). The composition of the nonlocal divergence operator $\mathcal{D}_{\delta}$ and its adjoint operator $\mathcal{D}_{\delta}^{*}$ are the only nonlocal operators needed to define the nonlocal operator $\mathcal{L}_{\delta}$ that operates on scalar-valued functions. However, other aspects of the nonlocal vector calculus, such as nonlocal vector identities and nonlocal operators acting on vector-valued functions, make use of additional nonlocal operators.

Thus, in addition to $\mathcal{D}_{\delta}$ and $\mathcal{D}_{\delta}^{*}$, we have the nonlocal gradient operator $\mathcal{G}_{\delta}$ defined by, for a scalar-valued function $\nu(\boldsymbol{x}, \boldsymbol{y})$,

$$
\left(\mathcal{G}_{\delta} \nu\right)(\boldsymbol{x}):=\int_{\Omega \cup \Omega_{\mathcal{I}_{\delta}}}(\nu(\boldsymbol{y}, \boldsymbol{x})+\nu(\boldsymbol{x}, \boldsymbol{y})) \boldsymbol{\alpha}_{\delta}(\boldsymbol{x}, \boldsymbol{y}) \mathcal{X}_{B_{\delta}(\boldsymbol{x})}(\boldsymbol{y}) \mathrm{d} \boldsymbol{y}
$$

for all $\boldsymbol{x} \in \Omega \cup \Omega_{\mathcal{I}_{\delta}}$ and its adjoint operator $\mathcal{G}_{\delta}^{*}$ given by, for a vector-valued function $\boldsymbol{u}(\boldsymbol{x})$,

$$
\left(\mathcal{G}_{\delta}^{*} \boldsymbol{u}\right)(\boldsymbol{x}, \boldsymbol{y})=-(\boldsymbol{u}(\boldsymbol{y})-\boldsymbol{u}(\boldsymbol{x})) \cdot \boldsymbol{\alpha}_{\delta}(\boldsymbol{x}, \boldsymbol{y}) \mathcal{X}_{B_{\delta}(\boldsymbol{x})}(\boldsymbol{y})
$$

for all $\boldsymbol{x}, \boldsymbol{y} \in \Omega \cup \Omega_{\mathcal{I}_{\delta}}$. Similarly, we have the nonlocal curl operator $\mathcal{C}_{\delta}$ 
defined by, for a vector-valued function $\boldsymbol{\nu}(\boldsymbol{x}, \boldsymbol{y})$,

$$
\left(\mathcal{C}_{\delta} \boldsymbol{\nu}\right)(\boldsymbol{x}):=\int_{\mathbb{R}^{d}}(\boldsymbol{\nu}(\boldsymbol{y}, \boldsymbol{x})+\boldsymbol{\nu}(\boldsymbol{x}, \boldsymbol{y})) \times \boldsymbol{\alpha}_{\delta}(\boldsymbol{x}, \boldsymbol{y}) \mathcal{X}_{B_{\delta}(\boldsymbol{x})}(\boldsymbol{y}) \mathrm{d} \boldsymbol{y}
$$

for all $\boldsymbol{x} \in \Omega \cup \Omega_{\mathcal{I}_{\delta}}$ and its adjoint operator $\mathcal{C}_{\delta}^{*}$ defined by, for a vector-valued function $\boldsymbol{u}(\boldsymbol{x})$,

$$
\left(\mathcal{C}_{\delta}^{*} \boldsymbol{u}\right)(\boldsymbol{x}, \boldsymbol{y})=-\boldsymbol{\alpha}_{\delta}(\boldsymbol{x}, \boldsymbol{y}) \times(\boldsymbol{u}(\boldsymbol{y})-\boldsymbol{u}(\boldsymbol{x})) \mathcal{X}_{B_{\delta}(\boldsymbol{x})}(\boldsymbol{y})
$$

for all $\boldsymbol{x}, \boldsymbol{y} \in \Omega \cup \Omega_{\mathcal{I}_{\delta}}$. Thus, in the nonlocal vector calculus we have pairs of divergence operators $\mathcal{D}_{\delta}$ and $-\mathcal{G}_{\delta}^{*}$, gradient operators $\mathcal{G}_{\delta}$ and $-\mathcal{D}_{\delta}^{*}$, and curl operators $\mathcal{C}_{\delta}$ and $\mathcal{C}_{\delta}^{*}$, with $\mathcal{D}_{\delta}, \mathcal{G}_{\delta}, \mathcal{C}_{\delta}$ acting on functions of two points, i.e. $\boldsymbol{\nu}(\boldsymbol{x}, \boldsymbol{y})$ and $\nu(\boldsymbol{x}, \boldsymbol{y})$, and $\mathcal{D}_{\delta}^{*}, \mathcal{G}_{\delta}^{*}, \mathcal{C}_{\delta}^{*}$ acting on functions of one point, i.e. $\boldsymbol{u}(\boldsymbol{x})$ and $u(\boldsymbol{x})$.

With these operators in hand, we have the nonlocal vector identities

$$
\left\{\begin{array}{l}
\mathcal{D}_{\delta}\left(\mathcal{C}_{\delta}^{*} \boldsymbol{u}\right)=0, \quad \mathcal{C}_{\delta}\left(\mathcal{G}_{\delta}^{*} u\right)=0 \\
\mathcal{D}_{\delta}\left(\mathcal{D}_{\delta}^{*} \boldsymbol{u}\right)=\mathcal{G}_{\delta}\left(\mathcal{G}_{\delta}^{*} \boldsymbol{u}\right)+\mathcal{C}_{\delta}\left(\mathcal{C}_{\delta}^{*} \boldsymbol{u}\right)
\end{array}\right.
$$

which are nonlocal analogues of $\nabla \cdot(\nabla \times \boldsymbol{u})=0, \nabla \times(\nabla u)=0$ and $\Delta \boldsymbol{u}=$ $\nabla \cdot(\nabla \boldsymbol{u})=\nabla(\nabla \cdot \boldsymbol{u})-\nabla \times(\nabla \times \boldsymbol{u})$, respectively. In particular, we can view $-\mathcal{D}_{\delta}\left(\mathcal{D}_{\delta}^{*} \boldsymbol{u}\right)=-\mathcal{G}_{\delta}\left(\mathcal{G}_{\delta}^{*} \boldsymbol{u}\right)-\mathcal{C}_{\delta}\left(\mathcal{C}_{\delta}^{*} \boldsymbol{u}\right)$ as a nonlocal vector Laplacian.

In addition, the operators introduced in this remark are used in D'Elia et al. $(2019 a)$ to define the nonlocal Hodge-Helmholtz decomposition of vector-valued functions that depend on two points $\boldsymbol{x}$ and $\boldsymbol{y}$. Note that the operators $\mathcal{D}_{\delta}, \mathcal{D}_{\delta}^{*}, \mathcal{G}_{\delta}$ and $\mathcal{G}_{\delta}^{*}$, as operators between functions of two points $\boldsymbol{x}$ and $\boldsymbol{y}$ and functions of one point $\boldsymbol{x}$ or $\boldsymbol{y}$, do not recover their classical counterparts in the local limit $\delta \rightarrow 0$. Thus, in parallel, additional examples of nonlocal operators acting on vector-valued functions of one point only are introduced in Du et al. (2013a) and further analysed in Mengesha and Du (2016), Du (2019), Du, Tao, Tian and Yang (2019c) and Lee and Du (2020). In fact, a nonlocal Helmholtz decomposition of a vector-valued function $\boldsymbol{u}(\boldsymbol{x})$ can be established which recovers the classical one in the local limit; see Lee and Du (2020). These operators can also be used to construct a nonlocal analogue of the Stokes equation, which was used to provide a foundation for the analysis of smoothed particle hydrodynamics (Du and Tian 2019, Lee and Du 2020). Some of these developments can be found in Section 20.

\section{Weak formulations of nonlocal models}

The model (1.5) is a strong formulation of a nonlocal problem that is required to hold pointwise on $\Omega \cup \Omega_{\mathcal{I}_{\delta}}$. For nonlocal problems subject to various inhomogeneous data, different weak formulations can be considered. In this section we consider one of the possible weak formulations corresponding 
12 M. D’Elia, Q. Du, C. Glusa, M. Gunzburger, X. Tian and Z. Zhou

to (1.5) and then relate that formulation to a minimization principle. We conclude the section by providing specific examples of the kernels $\gamma_{\delta}(\boldsymbol{x}, \boldsymbol{y})$ and their associated energy spaces.

\subsection{Function spaces, weak formulations and well-posedness}

\subsubsection{Function spaces and norms}

We define an 'energy' space

$$
V\left(\Omega \cup \Omega_{\mathcal{I}_{\delta}}\right)=\left\{v(\boldsymbol{x}) \in L^{2}\left(\Omega \cup \Omega_{\mathcal{I}_{\delta}}\right):\|v\|_{V\left(\Omega \cup \Omega_{\mathcal{I}_{\delta}}\right.}<\infty\right\},
$$

where $\|\cdot\|_{V\left(\Omega \cup \Omega_{\mathcal{I}_{\delta}}\right)}$ denotes the 'energy' norm defined as

$$
\begin{aligned}
& \|v\|_{V\left(\Omega \cup \Omega_{\mathcal{I}_{\delta}}\right)} \\
& \quad=\left(\int_{\Omega \cup \Omega_{\mathcal{I}_{\delta}}} \int_{\Omega \cup \Omega_{\mathcal{I}_{\delta}}}(v(\boldsymbol{y})-v(\boldsymbol{x}))^{2} \gamma_{\delta}(\boldsymbol{x}, \boldsymbol{y}) \mathrm{d} \boldsymbol{y} \mathrm{d} \boldsymbol{x}+\|v\|_{L^{2}\left(\Omega \cup \Omega_{\mathcal{I}_{\delta}}\right)}^{2}\right)^{1 / 2} .
\end{aligned}
$$

The space $V\left(\Omega \cup \Omega_{\mathcal{I}_{\delta}}\right)$ is by definition a subspace of $L^{2}\left(\Omega \cup \Omega_{\mathcal{I}_{\delta}}\right)$ and can be shown, for suitably chosen kernels, to be a Hilbert space that comes equipped with the inner product

$$
\begin{aligned}
& (u, v)_{V\left(\Omega \cup \Omega_{\mathcal{I}_{\delta}}\right)} \\
& =\int_{\Omega \cup \Omega_{\mathcal{I}_{\delta}}} \int_{\Omega \cup \Omega_{\mathcal{I}_{\delta}}}(v(\boldsymbol{y})-v(\boldsymbol{x}))(u(\boldsymbol{y})-u(\boldsymbol{x})) \gamma_{\delta}(\boldsymbol{x}, \boldsymbol{y}) \mathrm{d} \boldsymbol{y} \mathrm{d} \boldsymbol{x} \\
& \quad+(u, v)_{L^{2}\left(\Omega \cup \Omega_{\mathcal{I}_{\delta}}\right)} \quad \text { for all } u, v \in V\left(\Omega \cup \Omega_{\mathcal{I}_{\delta}}\right) .
\end{aligned}
$$

We also have the constrained energy space

$$
V_{c}\left(\Omega \cup \Omega_{\mathcal{I}_{\delta}}\right)=\left\{v(\boldsymbol{x}) \in V\left(\Omega \cup \Omega_{\mathcal{I}_{\delta}}\right): v(\boldsymbol{x})=0 \text { for } \boldsymbol{x} \in \Omega_{\mathcal{I}_{\delta}}\right\} .
$$

Under suitable conditions on the kernels, we can prove a nonlocal Poincaré inequality (Du et al. 2012a, Mengesha and Du 2013, Du 2019). Thus, we have that the seminorm

$$
|v|_{V_{c}\left(\Omega \cup \Omega_{\mathcal{I}_{\delta}}\right)}=\left(\int_{\Omega \cup \Omega_{\mathcal{I}_{\delta}}} \int_{\Omega \cup \Omega_{\mathcal{I}_{\delta}}}(v(\boldsymbol{y})-v(\boldsymbol{x}))^{2} \gamma_{\delta}(\boldsymbol{x}, \boldsymbol{y}) \mathrm{d} \boldsymbol{y} \mathrm{d} \boldsymbol{x}\right)^{1 / 2}
$$

is a norm on $V_{c}\left(\Omega \cup \Omega_{\mathcal{I}_{\delta}}\right)$ equivalent to the norm (2.2) with equivalence constants that are independent of $\delta$ as $\delta \rightarrow 0$. Correspondingly, $V_{c}\left(\Omega \cup \Omega_{\mathcal{I}_{\delta}}\right)$ is a Hilbert space equipped with the inner product

$$
(u, v)_{V_{c}\left(\Omega \cup \Omega_{\mathcal{I}_{\delta}}\right)}=\int_{\Omega \cup \Omega_{\mathcal{I}_{\delta}}} \int_{\Omega \cup \Omega_{\mathcal{I}_{\delta}}}(v(\boldsymbol{y})-v(\boldsymbol{x}))(u(\boldsymbol{y})-u(\boldsymbol{x})) \gamma_{\delta}(\boldsymbol{x}, \boldsymbol{y}) \mathrm{d} \boldsymbol{y} \mathrm{d} \boldsymbol{x}
$$

for all $u, v \in V_{c}\left(\Omega \cup \Omega_{\mathcal{I}_{\delta}}\right)$. For more detailed discussions of the conditions 
on the kernels and rigorous proofs of the properties on these spaces, we refer to Mengesha and Du $(2015,2016)$ and Du (2019).

To allow inhomogeneous data, we define the space $V_{t}\left(\Omega_{\mathcal{I}_{\delta}}\right)$,

$$
V_{t}\left(\Omega_{\mathcal{I}_{\delta}}\right)=\left\{v=\left.w\right|_{\Omega_{\mathcal{I}_{\delta}}} \text { for some } w \in V\left(\Omega \cup \Omega_{\mathcal{I}_{\delta}}\right)\right\}
$$

which involves restrictions to the domain $\Omega_{\mathcal{I}_{\delta}}$ having finite volume in $\mathbb{R}^{d}$. A norm on $V_{t}\left(\Omega_{\mathcal{I}_{\delta}}\right)$ can be defined by

$$
\|v\|_{V_{t}\left(\Omega_{\mathcal{I}_{\delta}}\right)}=\inf \left\{\|w\|_{V\left(\Omega \cup \Omega_{\mathcal{I}_{\delta}}\right)}\left|w \in V\left(\Omega \cup \Omega_{\mathcal{I}_{\delta}}\right), w\right|_{\Omega_{\mathcal{I}_{\delta}}}=v\right\} .
$$

One can view $V_{t}\left(\Omega_{\mathcal{I}_{\delta}}\right)$, induced by $V\left(\Omega \cup \Omega_{\mathcal{I}_{\delta}}\right)$, as a nonlocal analogue of the trace space $H^{1 / 2}(\partial \Omega)$ induced by $H^{1}(\Omega)$.

As mentioned in Remark 1.2, specific function spaces are defined by making specific choices for $\boldsymbol{\alpha}_{\delta}(\boldsymbol{x}, \boldsymbol{y})$. For some choices, the function spaces so defined can be shown to be equivalent to standard function spaces such as Sobolev spaces. See Section 2.2.1 for some examples.

\subsubsection{Weak formulations and well-posedness}

A weak formulation of problem (1.5) with $\mathcal{V} u=u$ is defined as follows:

Given $\gamma_{\delta}(\boldsymbol{x}, \boldsymbol{y})$ defined in (1.17) and given $g(\boldsymbol{x}) \in V_{t}\left(\Omega_{\mathcal{I}_{\delta}}\right)$ and $f(\boldsymbol{x}) \in V_{d}(\Omega)$, find $u(\boldsymbol{x}) \in V\left(\Omega \cup \Omega_{\mathcal{I}_{\delta}}\right)$ such that

$$
\begin{cases}\mathcal{A}_{\delta}(u, v)=\langle f, v\rangle & \text { for all } v \in V_{c}\left(\Omega \cup \Omega_{\mathcal{I}_{\delta}}\right), \\ u(\boldsymbol{x})=g(\boldsymbol{x}) & \text { for all } \boldsymbol{x} \in \Omega_{\mathcal{I}_{\delta}},\end{cases}
$$

where, for all $u(\boldsymbol{x}), v(\boldsymbol{x}) \in V\left(\Omega \cup \Omega_{\mathcal{I}_{\delta}}\right)$, we have the bilinear form

$$
\mathcal{A}_{\delta}(u, v)=\int_{\Omega \cup \Omega_{\mathcal{I}_{\delta}}} \int_{\Omega \cup \Omega_{\mathcal{I}_{\delta}}}(v(\boldsymbol{y})-v(\boldsymbol{x}))(u(\boldsymbol{y})-u(\boldsymbol{x})) \gamma_{\delta}(\boldsymbol{x}, \boldsymbol{y}) \mathrm{d} \boldsymbol{y} \mathrm{d} \boldsymbol{x}
$$

and, for all $f(\boldsymbol{x}) \in V_{d}(\Omega)$, the dual space of $V_{c}\left(\Omega \cup \Omega_{\mathcal{I}_{\delta}}\right)$ defined by extending the $L^{2}$ inner product as the duality pairing and with the induced norm $\|\cdot\|_{V_{d}(\Omega)}$, we have the linear functional

$$
\langle f, v\rangle=\int_{\Omega} f(\boldsymbol{x}) v(\boldsymbol{x}) \mathrm{d} \boldsymbol{x} .
$$

That (2.9)-(2.11) are indeed a weak formulation corresponding to (1.5) is an immediate consequence of setting $v(\boldsymbol{x})=0$ on $\Omega_{\mathcal{I}_{\delta}}$ in the nonlocal Green's first identity (1.22) so that the term involving $\mathcal{N}_{\delta} u$ vanishes. Equation (2.9) is a nonlocal analogue of the weak formulation of problem (1.1) with $\mathcal{B} u=u$ given by

$$
\int_{\Omega} \nabla v \cdot(\boldsymbol{D} \nabla u) \mathrm{d} \boldsymbol{x}=\langle f, v\rangle
$$

for all $v \in H_{0}^{1}(\Omega)$ along with $u=g$ on $\partial \Omega$. 
Under suitable conditions on the kernel $\gamma_{\delta}(\boldsymbol{x}, \boldsymbol{y})$, it can be shown that the bilinear form $\mathcal{A}_{\delta}(u, v)$ is symmetric and is continuous and coercive with respect to $V_{c}\left(\Omega \cup \Omega_{\mathcal{I}_{\delta}}\right)$ so that, by the Lax-Milgram theorem, problem (2.9) is well-posed. In particular, we have the a priori estimate with respect to the energy norm for the solution $u(\boldsymbol{x})$ of (2.9) given by

$$
\|u\|_{V\left(\Omega \cup \Omega_{\mathcal{I}_{\delta}}\right)} \leq C_{\delta}\left(\|f\|_{V_{d}(\Omega)}+\|g\|_{V_{t}\left(\Omega_{\mathcal{I}_{\delta}}\right)}\right),
$$

where the constant $C_{\delta}$ does not depend on $\delta$. Moreover, for suitably chosen kernels, one can consider its local $(\delta \rightarrow 0)$ and fractional $(\delta \rightarrow \infty)$ limits, as discussed in Part 2.

Remark 2.1 (a nonlocal Neumann model). We can of course also define a weak formulation of (1.5) for $\mathcal{V} u=\mathcal{N}_{\delta} u$, that is, for a nonlocal Neumann problem. In this case a weak formulation is given by: find $u \in$ $V\left(\Omega \cup \Omega_{\mathcal{I}_{\delta}}\right) \backslash \mathbb{R}$ such that

$$
\left\{\begin{array}{l}
\mathcal{A}_{\delta}(u, v)=\langle f, v\rangle+\int_{\Omega_{\mathcal{I}_{\delta}}} g(\boldsymbol{x}) v(\boldsymbol{x}) \mathrm{d} \boldsymbol{x} \quad \text { for all } v \in V\left(\Omega \cup \Omega_{\mathcal{I}_{\delta}}\right) \backslash \mathbb{R}, \\
\int_{\Omega} f(\boldsymbol{x}) \mathrm{d} \boldsymbol{x}+\int_{\Omega_{\mathcal{I}_{\delta}}} g(\boldsymbol{x}) \mathrm{d} \boldsymbol{x}=0
\end{array}\right.
$$

where $f \in V_{d}(\Omega)$ and for the Neumann problem $g(\boldsymbol{x})$ belongs to the dual space of $\left.V\left(\Omega \cup \Omega_{\mathcal{I}_{\delta}}\right)\right|_{\Omega_{\mathcal{I}_{\delta}}}$. In $(2.13)$, the quotient space $V\left(\Omega \cup \Omega_{\mathcal{I}_{\delta}}\right) \backslash \mathbb{R}$ is used to ensure uniqueness of solutions. The second equation in (2.13) is a compatibility condition on the data $f$ and $g$ that is necessary to ensure existence.

Equation (2.13) is a nonlocal analogue of the weak formulation corresponding to $(1.1)$ with $\mathcal{B} u=(\boldsymbol{D} \nabla u) \cdot \boldsymbol{n}$ given by

$$
\int_{\Omega} \nabla v \cdot(\boldsymbol{D} \nabla u) \mathrm{d} \boldsymbol{x}=\int_{\Omega} f v \mathrm{~d} \boldsymbol{x}+\int_{\partial \Omega} g v \mathrm{~d} \boldsymbol{x}
$$

for all $v \in H^{1}(\Omega) \backslash \mathbb{R}$ and a pair of prescribed data, also denoted by $f$ and $g$, that satisfy the compatibility condition

$$
\int_{\Omega} f \mathrm{~d} \boldsymbol{x}+\int_{\partial \Omega} g \mathrm{~d} \boldsymbol{x}=0 .
$$

We note that other ways of defining a nonlocal Neumann problem can also be used. For additional discussions about nonlocal Neumann problems, see e.g. Cortazar, Elgueta, Rossi and Wolanski (2008), Du (2019), Mengesha and Du (2015, 2016), Tao, Tian and Du (2017) and D'Elia, Tian and Yu (2019d). One can also find a fractional version of (1.5) with $\mathcal{V} u=\mathcal{N}_{\delta} u$ in Deng, Li, Tian and Zhan (2018) for a nonlocal Neumann problem of the fractional Laplacian in the bounded domain. In addition, for specialized power-like 
kernels, one can also relate this to problems associated with problems related to the regional Laplacian defined by (3.6).

For the sake of economy of exposition, we will focus on (2.9) in which $\mathcal{V} u=u$ and, at times, we will even focus on the homogeneous version of (2.9) for which $g(\boldsymbol{x})=0$.

\subsubsection{Relation to an energy minimization principle}

The weak formulation (2.9) can also be derived as the Euler-Lagrange equation for an energy minimization principle. Consider the energy functional

$$
\begin{aligned}
\mathcal{E}_{\delta}\left(u ; f, \gamma_{\delta}\right)= & \frac{1}{2} \int_{\Omega \cup \Omega_{\mathcal{I}_{\delta}}} \int_{\Omega \cup \Omega_{\mathcal{I}_{\delta}}}(u(\boldsymbol{y})-u(\boldsymbol{x}))^{2} \gamma_{\delta}(\boldsymbol{x}, \boldsymbol{y}) \mathrm{d} \boldsymbol{y} \mathrm{d} \boldsymbol{x} \\
& -\int_{\Omega} f(\boldsymbol{x}) u(\boldsymbol{x}) \mathrm{d} \boldsymbol{x}
\end{aligned}
$$

and the following minimization principle:

Given $\gamma_{\delta}(\boldsymbol{x}, \boldsymbol{y})$ defined in (1.17) and given $g(\boldsymbol{x}) \in V_{t}\left(\Omega_{\mathcal{I}_{\delta}}\right)$ and $f(\boldsymbol{x}) \in V_{d}(\Omega)$, find $u(\boldsymbol{x}) \in V\left(\Omega \cup \Omega_{\mathcal{I}_{\delta}}\right)$ such that

$$
\mathcal{E}_{\delta}\left(u ; f, \gamma_{\delta}\right) \text { is minimized }
$$

subject to $u(\boldsymbol{x})=g(\boldsymbol{x})$ for $\boldsymbol{x} \in \Omega_{\mathcal{I}_{\delta}}$.

Using standard techniques from the calculus of variations, one easily sees that (2.9) is indeed the Euler-Lagrange equation corresponding to the energy minimization principle (2.15) under suitable conditions on the kernel $\gamma_{\delta}(\boldsymbol{x}, \boldsymbol{y})$ and data $f$ and $g$.

\subsection{Kernel choices and the corresponding energy spaces}

We assume that $\gamma_{\delta}(\boldsymbol{x}, \boldsymbol{y})$ given by $(1.17)$ can be written in the form

$$
\gamma_{\delta}(\boldsymbol{x}, \boldsymbol{y})=\phi_{\delta}(\boldsymbol{x}, \boldsymbol{y}) \theta_{\delta}(\boldsymbol{x}, \boldsymbol{y}) \mathcal{X}_{B_{\delta}(\boldsymbol{x})}(\boldsymbol{y}),
$$

where $\theta_{\delta}(\boldsymbol{x}, \boldsymbol{y})$ and $\phi_{\delta}(\boldsymbol{x}, \boldsymbol{y})$ denote non-negative, symmetric, scalar-valued functions. This form is very general with respect to both operators and constitutive functions. In fact, by setting

$$
\boldsymbol{\alpha}_{\delta}(\boldsymbol{x}, \boldsymbol{y})=\frac{\boldsymbol{y}-\boldsymbol{x}}{|\boldsymbol{y}-\boldsymbol{x}|} \sqrt{\phi_{\delta}(\boldsymbol{x}, \boldsymbol{y})}
$$

and

$$
\theta_{\delta}(\boldsymbol{x}, \boldsymbol{y}):=\frac{\boldsymbol{y}-\boldsymbol{x}}{|\boldsymbol{y}-\boldsymbol{x}|} \cdot\left(\boldsymbol{\Theta}_{\delta}(\boldsymbol{x}, \boldsymbol{y}) \frac{\boldsymbol{y}-\boldsymbol{x}}{|\boldsymbol{y}-\boldsymbol{x}|}\right)
$$

in (1.17), we arrive at (2.16). Thus the only assumption made is that

$\boldsymbol{\alpha}_{\delta}(\boldsymbol{x}, \boldsymbol{y})$ is directed along the vector $\boldsymbol{y}-\boldsymbol{x}$. Note that $\boldsymbol{\alpha}_{\delta}(\boldsymbol{x}, \boldsymbol{y})$ given by (2.17) is indeed an antisymmetric function, whereas $\theta_{\delta}(\boldsymbol{x}, \boldsymbol{y})$ given by (2.18) 
is indeed a non-negative, symmetric function if $\boldsymbol{\Theta}_{\delta}(\boldsymbol{x}, \boldsymbol{y})$ is a symmetric in the function sense and symmetric and non-negative definite in the matrix sense.

We do not combine $\theta_{\delta}(\boldsymbol{x}, \boldsymbol{y})$ and $\phi_{\delta}(\boldsymbol{x}, \boldsymbol{y})$ into a single function because we want to keep separate the operator definition and constitutive roles played by $\gamma_{\delta}(\boldsymbol{x}, \boldsymbol{y})$; note that $\phi_{\delta}$ is only related to $\boldsymbol{\alpha}_{\delta}$ whereas $\theta_{\delta}$ is only related to $\boldsymbol{\Theta}_{\delta}$. Thus $\phi_{\delta}(\boldsymbol{x}, \boldsymbol{y})$ now takes over the role of $\boldsymbol{\alpha}_{\delta}(\boldsymbol{x}, \boldsymbol{y})$ in this regard, and $\theta_{\delta}(\boldsymbol{x}, \boldsymbol{y})$ can be viewed as a constitutive function. Note also that $\boldsymbol{\alpha}_{\delta}(\boldsymbol{x}, \boldsymbol{y})$ as given by (2.17) can be used to define the 'first-order' nonlocal operators $\mathcal{D}_{\delta}$ and $\mathcal{D}_{\delta}^{*}$, whereas $\gamma_{\delta}(\boldsymbol{x}, \boldsymbol{y})$ is used to define the 'second-order' nonlocal operator $\mathcal{L}_{\delta}$ and the 'Neumann' nonlocal operator $\mathcal{N}_{\delta}$. We will refer to $\gamma_{\delta}(\boldsymbol{x}, \boldsymbol{y})$ as the kernel, $\phi_{\delta}(\boldsymbol{x}, \boldsymbol{y})$ as the kernel function and $\theta_{\delta}(\boldsymbol{x}, \boldsymbol{y})$ as a constitutive function.

Remark 2.2 (scalar constitutive tensors). The case of $\boldsymbol{\Theta}_{\delta}(\boldsymbol{x}, \boldsymbol{y})$ being a scalar tensor (i.e. if $\boldsymbol{\Theta}_{\delta}(\boldsymbol{x}, \boldsymbol{y})=\theta_{\delta}(\boldsymbol{x}, \boldsymbol{y}) \boldsymbol{I}$, where $\boldsymbol{I}$ denotes the identity tensor and $\theta_{\delta}(\boldsymbol{x}, \boldsymbol{y})$ denotes a scalar-valued function) is by far the most common case considered, just as it is for a scalar diffusivity tensor $\boldsymbol{D}=a(\boldsymbol{x}) \boldsymbol{I}$ in the local PDE case. In this case, (2.18) becomes a tautology. Also, in this case, (1.17) simplifies to

$$
\begin{aligned}
\gamma_{\delta}(\boldsymbol{x}, \boldsymbol{y}) & =\theta_{\delta}(\boldsymbol{x}, \boldsymbol{y}) \boldsymbol{\alpha}_{\delta}(\boldsymbol{x}, \boldsymbol{y}) \cdot \boldsymbol{\alpha}_{\delta}(\boldsymbol{x}, \boldsymbol{y}) \mathcal{X}_{B_{\delta}(\boldsymbol{x})}(\boldsymbol{y}) \\
& =\theta_{\delta}(\boldsymbol{x}, \boldsymbol{y})\left|\boldsymbol{\alpha}_{\delta}(\boldsymbol{x}, \boldsymbol{y})\right|^{2} \mathcal{X}_{B_{\delta}(\boldsymbol{x})}(\boldsymbol{y})
\end{aligned}
$$

Remark 2.3 (homogeneous and inhomogeneous nonlocal operators). If $\boldsymbol{\Theta}_{\delta}(\boldsymbol{x}, \boldsymbol{y})=\boldsymbol{\Theta}_{\delta}(|\boldsymbol{y}-\boldsymbol{x}|)$ and $\phi_{\delta}(\boldsymbol{x}, \boldsymbol{y})=\phi_{\delta}(|\boldsymbol{y}-\boldsymbol{x}|)$, that is, $\boldsymbol{\Theta}_{\delta}$ and $\phi_{\delta}$ are radial functions, then (1.5) is a model for a homogeneous medium. This is not surprising because $\boldsymbol{\Theta}_{\delta}$ and $\phi_{\delta}$ being radial functions means that the nature of the interaction between a point $\boldsymbol{x}$ and another point $\boldsymbol{y}$ is independent of the location of the point $\boldsymbol{x}$; that interaction only depends on the distance $|\boldsymbol{y}-\boldsymbol{x}|$ between $\boldsymbol{x}$ and $\boldsymbol{y}$. In this case, (1.5) is a nonlocal analogue of the PDE $-\nabla \cdot(D \nabla u)=f$ in which $\boldsymbol{D}$ is a constant tensor. If in addition $\boldsymbol{\Theta}_{\delta}$ is a scalar tensor, (1.5) is a nonlocal analogue of the $\mathrm{PDE}-\kappa \Delta u=f$ in which $\kappa$ is a constant. Thus, to obtain an inhomogeneous model, both $\boldsymbol{\Theta}_{\delta}(\boldsymbol{x}, \boldsymbol{y})$ and $\phi_{\delta}(\boldsymbol{x}, \boldsymbol{y})$ cannot be radial functions. Note that $\phi_{\delta}(\boldsymbol{x}, \boldsymbol{y})$ is indeed often chosen to be a radial function, in which case $\boldsymbol{\Theta}_{\delta}(\boldsymbol{x}, \boldsymbol{y})$ has to be non-radial for $\mathcal{L}_{\delta}$ to be an inhomogeneous operator. It is important to note that a constant coefficient nonlocal problem does not mean that the kernel and constitutive functions are themselves constant functions; they merely need to be radial functions.

\subsubsection{A list of kernel functions in common use}

Because $\theta_{\delta}(\boldsymbol{x}, \boldsymbol{y})$ is a constitutive function which is not specific even within a single application, we focus on choices for the function $\phi_{\delta}(\boldsymbol{x}, \boldsymbol{y})$ that determ- 
Table 2.1. Kernel functions $\phi_{\delta}(\boldsymbol{x}, \boldsymbol{y})$ and the corresponding energy spaces $V\left(\Omega \cup \Omega_{\mathcal{I}_{\delta}}\right)$.

\begin{tabular}{lcl}
\hline \hline Type & Definition & Energy space $V\left(\Omega \cup \Omega_{\mathcal{I}_{\delta}}\right)$ \\
\hline Translation-invariant, integrable & $(2.19)$ & $L^{2}\left(\Omega \cup \Omega_{\mathcal{I}_{\delta}}\right)$ \\
Critical & $(2.20)$ & $\subsetneq L^{2}\left(\Omega \cup \Omega_{\mathcal{I}_{\delta}}\right)$ \\
Peridynamic & $(2.21)$ & $L^{2}\left(\Omega \cup \Omega_{\mathcal{I}_{\delta}}\right)$ for $d=2,3$ \\
Fractional & $(2.22)$ & $H^{s}\left(\Omega \cup \Omega_{\mathcal{I}_{\delta}}\right)$ \\
\hline
\end{tabular}

ines generic properties of a model. However, we again note that because we have assumed that $\boldsymbol{\Theta}_{\delta}(\boldsymbol{x}, \boldsymbol{y})$ is a symmetric, non-negative definite tensor, it follows that $\theta_{\delta}(\boldsymbol{x}, \boldsymbol{y})$ is a non-negative function for all $\boldsymbol{x}, \boldsymbol{y} \in \Omega \cup \Omega_{\mathcal{I}_{\delta}}$.

We also assume, as is usually the case, that for all $\boldsymbol{x}, \boldsymbol{y} \in \Omega \cup \Omega_{\mathcal{I}_{\delta}}$, the constitutive function $\theta_{\delta}(\boldsymbol{x}, \boldsymbol{y})$ is bounded from above and below by positive constants whose values do not depend on $\delta$. In fact, often $\theta_{\delta}(\boldsymbol{x}, \boldsymbol{y})$ does not depend on $\delta$. Thus we focus on a list of kernel functions $\phi_{\delta}(\boldsymbol{x}, \boldsymbol{y})$. In Table 2.1 we provide the energy spaces corresponding to the kernel functions we list.

Translation-invariant, integrable kernel functions. The kernel function is translation-invariant, that is, $\phi_{\delta}(\boldsymbol{x}, \boldsymbol{y})=\phi_{\delta}(\boldsymbol{y}-\boldsymbol{x})$, and satisfies for some positive constant $C>0$,

$$
C \leq \int_{\Omega \cup \Omega_{\mathcal{I}_{\delta}}} \phi_{\delta}(\boldsymbol{y}-\boldsymbol{x}) \mathrm{d} \boldsymbol{y}<\infty \quad \text { for all } \boldsymbol{x} \in \Omega \cup \Omega_{\mathcal{I}_{\delta}} .
$$

In the design of numerical methods, one may need to differentiate between cases for which

- $\phi_{\delta}(\boldsymbol{z})$ is a smooth, bounded function of $\boldsymbol{z}$,

- $\phi_{\delta}(\boldsymbol{z})$ is a bounded function of $\boldsymbol{z}$ but is not smooth, e.g. a piecewise constant function,

- $\phi_{\delta}(\boldsymbol{z})$ is a singular but integrable function, e.g. $\phi_{\delta}(\boldsymbol{z}) \propto 1 /|\boldsymbol{z}|^{r}$ with $r<d$.

A special case of translation-invariant kernel functions is that of radial kernel functions for which $\phi_{\delta}(\boldsymbol{z})=\phi_{\delta}(|\boldsymbol{z}|)$.

'Critical' kernel functions. The kernel function satisfies

$$
\phi_{\delta}(\boldsymbol{x}, \boldsymbol{y}) \propto \frac{1}{|\boldsymbol{y}-\boldsymbol{x}|^{d}} .
$$

We refer to this kernel as critical because it 'just misses being integrable', that is, $1 /|\boldsymbol{y}-\boldsymbol{x}|^{d-\epsilon}$ is integrable for any $\epsilon>0$ and for any $d$, but is not 
integrable for any $d$ if $\epsilon=0$. For the other kernels in this list, the corresponding energy space is precisely known; see Table 2.1. For critical kernels, that is not the case; what is known is that energy space is a Hilbert space and is a strict subspace of $L^{2}\left(\Omega \cup \Omega_{\mathcal{I}_{\delta}}\right)$.

'Peridynamic' kernel functions. The kernel function satisfies

$$
\phi_{\delta}(\boldsymbol{x}, \boldsymbol{y}) \propto \frac{1}{|\boldsymbol{y}-\boldsymbol{x}|} .
$$

We refer to this kernel as peridynamic because it has the same singular behaviour as one of the commonly used kernels in peridynamic models for solid mechanics. Note that for $d=1$ this kernel function is a member of the critical class, whereas for $d=2$ it is integrable and for $d=3$ it is square-integrable.

Fractional kernel functions. The kernel function satisfies

$$
\phi_{\delta}(\boldsymbol{x}, \boldsymbol{y}) \propto \frac{1}{|\boldsymbol{y}-\boldsymbol{x}|^{d+2 s}} .
$$

We refer to this kernel as fractional because the singularity at $\boldsymbol{y}=\boldsymbol{x}$ is that of the kernel for the fractional Laplace operator. This kernel plays a central role in Section 3.

Remark 2.4. We note that many square-integrable kernel functions of the more general form $\phi_{\delta}(\boldsymbol{x}, \boldsymbol{y})$ also have $V\left(\Omega \cup \Omega_{\mathcal{I}_{\delta}}\right)=L^{2}\left(\Omega \cup \Omega_{\mathcal{I}_{\delta}}\right)$ as the energy space. However, we do not consider this class of kernels in the later sections.

Remark 2.5 (solution smoothness in nonlocal diffusion). For translation-invariant integrable kernel functions such as (2.19), the solution may not be smoother than the data. For example, if $g(\boldsymbol{x})=0$ and $f(\boldsymbol{x}) \in L^{2}(\Omega)$, then generically, the solution $u(\boldsymbol{x})=\left(-\mathcal{L}_{\delta}\right)^{-1} f \in L^{2}\left(\Omega \cup \Omega_{\mathcal{I}_{\delta}}\right)$. Because $L^{2}(\Omega)$ includes, for example, functions with jump discontinuities possibly over some co-dimension 1 interfaces, this means that if the data $f(\boldsymbol{x})$ has a jump discontinuity, so may the solution $u(\boldsymbol{x})$. For the fractional kernel function, we could have $2 s$ derivatives of smoothing, that is, if the data $f(\boldsymbol{x}) \in H^{-s}(\Omega)$, then $u(\boldsymbol{x}) \in H^{s}\left(\Omega \cup \Omega_{\mathcal{I}_{\delta}}\right)$, where $H^{s}\left(\Omega \cup \Omega_{\mathcal{I}_{\delta}}\right)$ denotes a fractional Sobolev space and $H^{-s}(\Omega)$ its dual space. Note that for $s<1 / 2$, $H^{s}\left(\Omega \cup \Omega_{\mathcal{I}_{\delta}}\right)$ also contains functions having jump discontinuities.

Remark 2.6 (scaling constants and limit behaviour). Often kernel functions take the form

$$
\phi_{\delta}(\boldsymbol{x}, \boldsymbol{y})=C \varphi(\boldsymbol{x}, \boldsymbol{y})
$$

where $C$ denotes a scaling constant that, depending on the specific instance, may depend on $\delta$ and also on other parameters appearing in the definition of 
$\phi_{\delta}(\boldsymbol{x}, \boldsymbol{y})$. One approach towards defining the scaling constant $C$ is to choose it so that in the limit $\delta \rightarrow 0$, a nonlocal model and its solution reduce to a local PDE model and its solution, respectively. Another approach is to choose $C$ so that in the limit $\delta \rightarrow \infty$, the nonlocal model and its solution reduce to a model that is posed on $\mathbb{R}^{d}$. Below, we will have occasion to follow both approaches. In any case, the scaling constant is a modelling choice so that, for example, if neither type of limiting behaviour is of interest, then the scaling constant should be chosen based on the physics being modelled.

\section{Fractional diffusion models}

In this section we consider a widely used nonlocal diffusion model that involves the fractional Laplace operator $-(-\Delta)^{s}$ with $s \in(0,1)$. In a real sense, fractional diffusion models can be thought of as being a special case of the general nonlocal diffusion models discussed in Sections 1 and 2. After all, fractional diffusion models are defined in terms of a specific choice for the kernel function; see (2.22). On the other hand, the scope of fractional diffusion models and the literature devoted to their analysis, approximation and application hugely dwarf what is available for other nonlocal diffusion models. Thus a more detailed consideration of fractional models is warranted here.

The integral definition of the fractional Laplacian, for $u(\boldsymbol{x}): \mathbb{R}^{d} \rightarrow \mathbb{R}^{d}$ with $d \in \mathbb{N}_{+}$, is given by

$$
(-\Delta)^{s} u(\boldsymbol{x})=\int_{\mathbb{R}^{d}}(u(\boldsymbol{x})-u(\boldsymbol{y})) \gamma_{s}(\boldsymbol{x}, \boldsymbol{y}) \mathrm{d} \boldsymbol{y} \quad \text { for all } \boldsymbol{x} \in \mathbb{R}^{d}
$$

with

$$
\gamma_{s}(\boldsymbol{x}, \boldsymbol{y})=\frac{C_{d, s}}{|\boldsymbol{y}-\boldsymbol{x}|^{d+2 s}}
$$

Note that this is a case in which the integral in (3.1) should be interpreted in the principal value sense. In (3.1), the constant $C_{d, s}$ is given by

$$
C_{d, s}=\frac{2^{2 s} s \Gamma(s+d / 2)}{\pi^{d / 2} \Gamma(1-s)},
$$

where $\Gamma$ denotes the gamma function.

The integral representation of the fractional Laplacian given in (3.1) can be viewed as a special case of the nonlocal Laplacian given by (1.6). It is also equivalent to and can be directly derived from the Fourier representation (Valdinoci 2009)

$$
\begin{aligned}
(-\Delta)^{s} u(\boldsymbol{x}) & =\frac{1}{(2 \pi)^{d}} \int_{\mathbb{R}^{d}}|\boldsymbol{\xi}|^{2 s}\left(u, \mathrm{e}^{-\mathrm{i} \boldsymbol{\xi} \cdot \boldsymbol{x}}\right) \mathrm{e}^{\mathrm{i} \boldsymbol{\xi} \cdot \boldsymbol{x}} \mathrm{d} \boldsymbol{\xi} \\
& =\mathcal{F}^{-1}\left(|\boldsymbol{\xi}|^{2 s} \mathcal{F}\{u\}(\boldsymbol{\xi})\right)(\boldsymbol{x}),
\end{aligned}
$$


where $\mathcal{F}$ denotes the Fourier transform. If $s=1$, the spectral operator (3.4) coincides with the usual PDE Laplacian $-\Delta$, whereas it reproduces the identity operator when $s=0$. In fact, it is well known that $(-\Delta)^{s} u(x) \rightarrow$ $u(x)$ as $s \rightarrow 0^{+}$and $(-\Delta)^{s} u(x) \rightarrow-\Delta u(x)$ as $s \rightarrow 1^{-}$for a regular function $u$; see e.g. Stinga (2019, Theorems 3 and 4) and Di Nezza, Palatucci and Valdinoci (2012, Proposition 4.4).

The fractional Laplacian (3.1) is often used to model superdiffusion for which the mean-squared particle displacement grows faster than that for PDE models of diffusion. At microscopic scales, in contrast to standard diffusion that is described by Brownian motion, superdiffusion can be described by Lévy flights in which the length of particle jumps follows a heavy-tailed distribution, reflecting the long-range interactions between particles. See e.g. Metzler and Klafter (2000) and Sokolov, Klafter and Blumen (2002) for discussions of the physical background and practical applications of anomalous diffusion.

\subsection{Integral fractional Laplacian models for diffusion on bounded domains}

Letting $\Omega \subset \mathbb{R}^{d}$ denote a bounded Lipschitz domain, we define the integral fractional Laplacian $-(-\Delta)^{s}$ to be the restriction of the full-space operator to functions satisfying a volume constraint on $\Omega_{\mathcal{I}_{\infty}}:=\mathbb{R}^{d} \backslash \Omega$, i.e. on the interaction domain corresponding to $\Omega$. The fractional Poisson problem is then given by

$$
\begin{cases}(-\Delta)^{s} u=f & \text { for all } \boldsymbol{x} \in \Omega, \\ u=g & \text { for all } \boldsymbol{x} \in \Omega_{\mathcal{I}_{\infty}},\end{cases}
$$

where we have a given source term $f(\boldsymbol{x})$ and Dirichlet volume constraint data $g(\boldsymbol{x})$ defined for $\boldsymbol{x} \in \Omega$ and $\boldsymbol{x} \in \Omega_{\mathcal{I}_{\infty}}$, respectively. Problem (3.5) is a nonlocal analogue of the local Poisson problem for the PDE Laplacian $\Delta$. It is known (see e.g. Biccari and Hernández-Santamaría 2018) that as $s \rightarrow 1^{-}$, the solution of integral fractional diffusion model (3.5) strongly converges to the solution of the local diffusion problem in $H^{1-\epsilon}(\Omega)$.

A related operator on $\Omega$ is the regional fractional Laplacian (Bogdan, Burdzy and Chen 2003, Chen and Kim 2002)

$$
-(-\Delta)_{\text {regional }}^{s} u(\boldsymbol{x})=C_{d, s} \int_{\Omega} \frac{u(\boldsymbol{y})-u(\boldsymbol{x})}{|\boldsymbol{y}-\boldsymbol{x}|^{d+2 s}} \mathrm{~d} \boldsymbol{y},
$$

which is used in one of several approaches for generalizing the PDE Poisson problem with a homogeneous Neumann boundary condition to the fractional Laplacian case (Dipierro, Ros-Oton and Valdinoci 2017). Note that in (3.5) the operator has not changed, that is, the operator defined in (3.1) is used; what has changed is that domain of the operator is changed from $\mathbb{R}^{d}$ to the bounded domain $\Omega$. On the other hand, in using the operator in (3.6), not 
only is the domain changed in the same manner, but the operator itself has changed, that is, (3.1) involves an integral over $\mathbb{R}^{d}$ whereas (3.6) involves an integral over $\Omega$.

To discuss the variational form of the fractional Poisson problem (3.5), we use the standard fractional Sobolev space $H^{s}\left(\mathbb{R}^{d}\right)$ defined via the Fourier transform as

$$
H^{s}\left(\mathbb{R}^{d}\right)=\left\{u \in L^{2}\left(\mathbb{R}^{d}\right): \int_{\mathbb{R}^{d}}\left(1+|\boldsymbol{\xi}|^{2 s}\right)|\mathcal{F} u(\boldsymbol{\xi})|^{2} \mathrm{~d} \boldsymbol{\xi}<\infty\right\} .
$$

If $\Omega \subset \mathbb{R}^{d}$ is a bounded domain, we define the Sobolev space $H^{s}(\Omega)$ as (McLean 2000)

$$
H^{s}(\Omega):=\left\{u \in L^{2}(\Omega):\|u\|_{H^{s}(\Omega)}<\infty\right\}
$$

equipped with the norm

$$
\|u\|_{H^{s}(\Omega)}^{2}=|u|_{H^{s}(\Omega)}^{2}+\|u\|_{L^{2}(\Omega)}^{2},
$$

where we have the seminorm

$$
|u|_{H^{s}(\Omega)}^{2}=\int_{\Omega} \int_{\Omega} \frac{(u(\boldsymbol{y})-u(\boldsymbol{x}))^{2}}{|\boldsymbol{y}-\boldsymbol{x}|^{d+2 s}} \mathrm{~d} \boldsymbol{y} \mathrm{d} \boldsymbol{x} .
$$

Moreover, when imposing a homogeneous Dirichlet volume constraints, e.g. $g=0$ in (3.5), we use the space

$$
H_{c}^{s}(\Omega):=\left\{u \in H^{s}\left(\mathbb{R}^{d}\right): u=0 \text { for all } \boldsymbol{x} \in \Omega_{\mathcal{I}_{\infty}}\right\}
$$

that is equipped with the norm

$$
\|u\|_{H_{c}^{s}(\Omega)}^{2}=\|u\|_{H^{s}\left(\mathbb{R}^{d}\right)}^{2}=\|u\|_{L^{2}(\Omega)}^{2}+|u|_{H^{s}\left(\mathbb{R}^{d}\right)}^{2} .
$$

For $s>1 / 2, H_{c}^{s}(\Omega)$ coincides with the space $H_{0}^{s}(\Omega)$ that is the closure of $C_{0}^{\infty}(\Omega)$ with respect to the $H^{s}(\Omega)$-norm, whereas for $s<1 / 2, H_{c}^{s}(\Omega)$ is identical to $H^{s}(\Omega)$. In the critical case $s=1 / 2, H_{c}^{s}(\Omega) \subsetneq H_{0}^{s}(\Omega)$. See e.g. McLean (2000, Chapter 3) for a detailed discussion.

A variational form of (3.5) is derived starting from the integration-byparts formula (e.g. the nonlocal Green's first identity (1.22))

$$
\begin{aligned}
& \frac{1}{2} \int_{\mathbb{R}^{d}} \int_{\mathbb{R}^{d}}(u(\boldsymbol{y})-u(\boldsymbol{x}))(v(\boldsymbol{y})-v(\boldsymbol{x})) \gamma_{s}(\boldsymbol{x}, \boldsymbol{y}) \mathrm{d} \boldsymbol{y} \mathrm{d} \boldsymbol{x} \\
& \quad=\int_{\Omega}\left((-\Delta)^{s} u(\boldsymbol{x})\right) v(\boldsymbol{x}) \mathrm{d} \boldsymbol{x}+\int_{\Omega_{\mathcal{I}_{\infty}}}\left(\mathcal{N}_{s} u(\boldsymbol{x})\right) v(\boldsymbol{x}) \mathrm{d} \boldsymbol{x},
\end{aligned}
$$

where, as in (1.12), we have the nonlocal operator given by

$$
\mathcal{N}_{s} u(\boldsymbol{x})=\int_{\mathbb{R}^{d}}(u(\boldsymbol{x})-u(\boldsymbol{y})) \gamma_{s}(\boldsymbol{x}, \boldsymbol{y}) \mathrm{d} \boldsymbol{y} \quad \text { for all } \boldsymbol{x} \in \Omega_{\mathcal{I}_{\infty}} .
$$

An alternative definition of $\mathcal{N}_{s}$ has been presented in Dipierro et al. (2017), 
corresponding to a nonlocal operator $\mathcal{N}_{\delta}$ that is defined on $\Omega_{\delta}$ as an integral over the domain $\Omega$ only, instead of $\Omega \cup \Omega_{\mathcal{I}_{\delta}}$ as in the case of (1.10). Substituting the volume constraint in $(3.5)$ and $v(\boldsymbol{x})=0$ on $\Omega_{\mathcal{I}_{\infty}}$, we then have the following weak formulation:

Find $u(\boldsymbol{x}) \in H^{s}(\Omega)$ such that $u(\boldsymbol{x})=g(\boldsymbol{x})$ for all $\boldsymbol{x} \in \Omega_{\mathcal{I}_{\infty}}$ and

$$
\mathcal{A}_{s}(u, v)=\langle f, v\rangle+(g, v)_{\Omega, \Omega_{\mathcal{I}_{\infty}}} \text { for all } v \in H_{c}^{s}(\Omega),
$$

where

$$
\begin{aligned}
& \mathcal{A}_{s}(u, v)=\underbrace{\frac{1}{2} \int_{\Omega} \int_{\Omega}(u(\boldsymbol{y})-u(\boldsymbol{x}))(v(\boldsymbol{y})-v(\boldsymbol{x})) \gamma_{s}(\boldsymbol{x}, \boldsymbol{y}) \mathrm{d} \boldsymbol{y} \mathrm{d} \boldsymbol{x}}_{\mathcal{A}_{\Omega, \Omega}(u, v)} \\
& +\underbrace{\int_{\Omega} u(\boldsymbol{x}) v(\boldsymbol{x}) \int_{\Omega_{\mathcal{I}_{\infty}}} \gamma_{s}(\boldsymbol{x}, \boldsymbol{y}) \mathrm{d} \boldsymbol{y} \mathrm{d} \boldsymbol{x}}_{\mathcal{A}_{\Omega, \Omega_{\mathcal{I}_{\infty}}}(u, v)}
\end{aligned}
$$

and

$$
(g, v)_{\Omega, \Omega_{\mathcal{I}_{\infty}}}=\int_{\Omega} v(\boldsymbol{x}) \int_{\Omega_{\mathcal{I}_{\infty}}} g(\boldsymbol{y}) \gamma_{s}(\boldsymbol{x}, \boldsymbol{y}) \mathrm{d} \boldsymbol{y} \mathrm{d} \boldsymbol{x} .
$$

The bilinear form $\mathcal{A}_{s}(\cdot, \cdot)$ is $H_{c}^{s}(\Omega)$-coercive and continuous so that as long as the right-hand side of $(3.7)$ is bounded, the well-posedness of that problem follows from the Lax-Milgram theorem. If $g=0, \mathcal{A}_{s}(\cdot, \cdot)$ is identical to the bilinear form $\mathcal{A}_{\delta}(\cdot, \cdot)$ defined in $(2.10)$ with $\delta=\infty$. On the other hand, for $g \neq 0, \mathcal{A}_{s}(\cdot, \cdot)$ can be seen as the $\delta=\infty$ limit of the bilinear form corresponding to some inhomogeneously constrained nonlocal diffusion problems discussed in Du (2019), instead of the bilinear form $\mathcal{A}_{\delta}(\cdot, \cdot)$ given by (2.10). Note that the bilinear form $\mathcal{A}_{\Omega, \Omega}(\cdot, \cdot)$ can be seen to correspond to the regional fractional Laplacian.

Note that

$$
\mathcal{A}_{\Omega, \Omega_{\mathcal{I}_{\infty}}}(u, v)=C_{d, s} \int_{\Omega} u(\boldsymbol{x}) v(\boldsymbol{x})\left(\int_{\Omega_{\mathcal{I}_{\infty}}} \frac{1}{|\boldsymbol{y}-\boldsymbol{x}|^{d+2 s}} \mathrm{~d} \boldsymbol{y}\right) \mathrm{d} \boldsymbol{x} .
$$

The identity

$$
\frac{1}{|\boldsymbol{y}-\boldsymbol{x}|^{d+2 s}}=-\frac{1}{2 s} \nabla_{\boldsymbol{y}} \cdot \frac{\boldsymbol{y}-\boldsymbol{x}}{|\boldsymbol{y}-\boldsymbol{x}|^{d+2 s}}
$$

and Gauss's theorem results in

$$
\mathcal{A}_{\Omega, \Omega_{\mathcal{I}_{\infty}}}(u, v)=\frac{C_{d, s}}{2 s} \int_{\Omega} u(\boldsymbol{x}) v(\boldsymbol{x})\left(\int_{\partial \Omega} \frac{(\boldsymbol{y}-\boldsymbol{x}) \cdot \boldsymbol{n}_{\boldsymbol{y}}}{|\boldsymbol{y}-\boldsymbol{x}|^{d+2 s}} \mathrm{~d} \boldsymbol{y}\right) \mathrm{d} \boldsymbol{x},
$$

where $\boldsymbol{n}_{\boldsymbol{y}}$ denotes the unit outer normal to $\partial \Omega$ at $\boldsymbol{y}$. We then have that

$$
\mathcal{A}_{s}(u, v)=\mathcal{A}_{\Omega, \Omega}(u, v)+\frac{C_{d, s}}{2 s} \int_{\Omega} u(\boldsymbol{x}) v(\boldsymbol{x})\left(\int_{\partial \Omega} \frac{(\boldsymbol{y}-\boldsymbol{x}) \cdot \boldsymbol{n}_{\boldsymbol{y}}}{|\boldsymbol{y}-\boldsymbol{x}|^{d+2 s}} \mathrm{~d} \boldsymbol{y}\right) \mathrm{d} \boldsymbol{x}
$$


so that an integral over the unbounded domain $\Omega_{\mathcal{I}_{\infty}}$ can be avoided in any computation involving $\mathcal{A}_{s}(u, v)$. Of course, for a homogeneous volume constraint $g(\boldsymbol{x})=0$ on $\Omega_{\mathcal{I}_{\infty}}$, the right-hand side in (3.7) also only involves integrals over $\Omega$. For $g(\boldsymbol{x}) \neq 0$, one does have to evaluate, for $\boldsymbol{x} \in \Omega$, the data integral $\int_{\Omega_{\mathcal{I}_{\infty}}} g(\boldsymbol{y}) \gamma_{s}(\boldsymbol{x}, \boldsymbol{y}) \mathrm{d} \boldsymbol{y}$ that, in principle, can be approximated using a quadrature rule.

Above we have incorporated the inhomogeneous volume constraint directly into the weak form. An alternative is to enforce that condition via Lagrange multipliers (Acosta, Borthagaray and Heuer 2019).

\subsubsection{Truncated interaction domains}

An alternative to the approach based on the bilinear form (3.10) is to truncate the integration domain $\Omega_{\mathcal{I}_{\infty}}$ as is considered in D'Elia and Gunzburger (2013). For $\delta>\operatorname{diam}(\Omega)$, we pose the truncated problem

$$
\begin{cases}(-\Delta)_{\delta}^{s} u=f & \text { for all } \boldsymbol{x} \in \Omega, \\ u=g & \text { for all } \boldsymbol{x} \in \Omega_{\mathcal{I}_{\delta}}\end{cases}
$$

where

$$
(-\Delta)_{\delta}^{s} u(\boldsymbol{x}):=\int_{\Omega \cup \Omega_{\mathcal{I}_{\delta}}}(u(\boldsymbol{x})-u(\boldsymbol{y})) \gamma_{s}(\boldsymbol{x}, \boldsymbol{y}) \mathrm{d} \boldsymbol{y} .
$$

D'Elia and Gunzburger (2013) and Burkovska and Gunzburger (2019b) have shown that if $u_{\infty}$ and $u_{\delta}$ denote the solutions of (3.5) and (3.11), respectively, then

$$
\left\|u_{\delta}-u_{\infty}\right\|_{H^{s}(\Omega)} \leq \frac{C}{\delta^{2 s}} \text { as } \delta \rightarrow \infty,
$$

where $C$ depends on norms of $f$ and $g$.

Remark 3.1. Clearly, (3.12) implies that the solution of (3.11) can be viewed as an approximation of the solution of (3.5). However, (3.11) and its solution are useful in their own right. In (3.5), the horizon $\delta$ is infinite, but, in practice, although $\delta$ may be large, it is not likely to be infinite. In (3.11), $\delta$ is assumed large but finite so that it may provide a better model for practical applications that feature large horizons. In this case one can reverse roles and interpret the solution of (3.5) to be an approximation of the solution of (3.11), with (3.12) now telling us something about the error incurred by replacing the perhaps more useful model (3.11) with the much more studied model (3.5).

\subsection{Spectral fractional Laplacian models for diffusion on bounded domains}

An alternative definition of a fractional-order Laplacian on a bounded domain $\Omega$ makes use of spectral information about the PDE Laplacian. Here we focus on the case of homogeneous constraints. 
Let $0<\lambda_{0} \leq \lambda_{1} \leq \cdots$ and $\varphi_{0}, \varphi_{1}, \ldots$ denote the eigenvalues and the corresponding eigenfunctions of the PDE Laplacian $-\Delta$ with a homogeneous Dirichlet boundary condition, that is, we have

$$
\begin{cases}-\Delta \varphi_{m}(\boldsymbol{x})=\lambda_{m} \varphi_{m}(\boldsymbol{x}) & \text { for all } \boldsymbol{x} \in \Omega \\ \varphi_{m}(\boldsymbol{x})=0 & \text { for all } \boldsymbol{x} \in \partial \Omega\end{cases}
$$

where the orthogonal eigenfunctions are normalized so that $\left\|\varphi_{m}\right\|_{L^{2}(\Omega)}=1$. Then the eigenfunctions $\left\{\varphi_{m}\right\}_{m=0}^{\infty}$ form a complete orthonormal basis for $L^{2}(\Omega)$. As a result, any function $u \in L^{2}(\Omega)$ can be expanded as

$$
u=\sum_{m=0}^{\infty} u_{m} \varphi_{m} \quad \text { with } u_{m}=\left(u, \varphi_{m}\right)
$$

where $(\cdot, \cdot)$ denotes the inner product of $L^{2}(\Omega)$. We then have

$$
(-\Delta) u(\boldsymbol{x})=\sum_{m=0}^{\infty} u_{m} \lambda_{m} \varphi_{m}(\boldsymbol{x})
$$

and the spectral fractional Laplacian of order $s \in(0,1)$ with homogeneous boundary condition is given by

$$
\left(-\left.\Delta\right|_{\Omega, 0}\right)^{s} u(\boldsymbol{x})=\sum_{m=0}^{\infty} u_{m} \lambda_{m}^{s} \varphi_{m}(\boldsymbol{x}) .
$$

As $s \rightarrow 0$, the identity operator is recovered, whereas the integer order Laplacian is recovered as $s \rightarrow 1$.

For a given source term $f$, the spectral fractional Laplacian Poisson problem for $u(\boldsymbol{x}), \boldsymbol{x} \in \Omega$, is then given by

$$
\left(-\left.\Delta\right|_{\Omega, 0}\right)^{s} u(\boldsymbol{x})=f(\boldsymbol{x}) \quad \text { for all } \boldsymbol{x} \in \Omega .
$$

Using the heat kernel $p_{\Omega, 0}(\boldsymbol{x}, \boldsymbol{y}, t)$, the spectral fractional Laplacian can be rewritten (see e.g. Abatangelo and Dupaigne 2017) in the form of the nonlocal operator

$$
\left(-\left.\Delta\right|_{\Omega, 0}\right)^{s} u(\boldsymbol{x})=\int_{\Omega} \gamma_{\Omega, 0 ; s}(\boldsymbol{x}, \boldsymbol{y})(u(\boldsymbol{y})-u(\boldsymbol{x})) \mathrm{d} \boldsymbol{y}+\kappa_{\Omega, 0 ; s}(\boldsymbol{x}) u(\boldsymbol{x}),
$$

where

$$
\begin{aligned}
\gamma_{\Omega, 0 ; s}(\boldsymbol{x}, \boldsymbol{y}) & =\frac{s}{\Gamma(1-s)} \int_{0}^{\infty} p_{\Omega, 0}(t, \boldsymbol{x}, \boldsymbol{y}) \frac{1}{t^{1+s}} \mathrm{~d} t \\
\kappa_{\Omega, 0 ; s}(\boldsymbol{x}) & =\frac{s}{\Gamma(1-s)} \int_{0}^{\infty}\left(1-\int_{\Omega} p_{\Omega, 0}(t, \boldsymbol{x}, \boldsymbol{y}) \mathrm{d} \boldsymbol{y}\right) \frac{1}{t^{1+s}} \mathrm{~d} t
\end{aligned}
$$


If $\Omega=\mathbb{R}^{d}$, the integral and the spectral definitions of the fractional Laplacian coincide, but they are different for bounded domains (Servadei and Valdinoci 2014). For example, in the particular case of $\Omega=\mathbb{R}_{+}^{d}$, i.e. the half-plane, the kernel of the spectral fractional Laplacian is given by

$$
\gamma_{\mathbb{R}^{d}, 0 ; s}(\boldsymbol{x}, \boldsymbol{y})=\gamma_{s}(\boldsymbol{x}, \boldsymbol{y})-\gamma_{s}(\boldsymbol{x},-\boldsymbol{x}) \neq \gamma_{s}(\boldsymbol{x}, \boldsymbol{y}) \quad \text { for all } \boldsymbol{x}, \boldsymbol{y} \in \mathbb{R}^{d}{ }_{+} \text {. }
$$

Note that the spectral definition can allow for the treatment of nonhomogeneous boundary conditions of various types; see e.g. Antil, Pfefferer and Rogovs (2018) and Cusimano, del Teso, Gerardo-Giorda and Pagnini (2018).

\subsection{Additional considerations about fractional Laplacian models}

\subsubsection{Other integral representations of the fractional Laplacian}

The Dunford-Taylor integral is a powerful tool in the numerical analysis of the fractional diffusion problem (Bonito and Pasciak 2015, Bonito and Pasciak 2017, Bonito, Lei and Pasciak 2019). In particular, the solution $u$ of the Poisson problem (3.14) involving the spectral fractional Laplacian can be given by the Dunford-Taylor representation

$$
u=\left(-\left.\Delta\right|_{\Omega, 0}\right)^{-s} f=\frac{\sin (s \pi)}{\pi} \int_{0}^{\infty} \mu^{-s}(\mu-\Delta)^{-1} f \mathrm{~d} \mu .
$$

A combination of using a sinc quadrature rule and a finite element discretization of the reaction-diffusion-type term in the integrand was explored by Bonito and Pasciak $(2015,2017)$.

Using similar techniques, the bilinear form $\mathcal{A}_{s}(\cdot, \cdot)$ associated with the integral fractional Laplacian can be rewritten as (Bonito et al. 2019)

$$
\mathcal{A}_{s}(u, v)=\frac{2 \sin (\pi s)}{\pi} \int_{0}^{\infty} \mu^{1-2 s} \int_{\mathbb{R}^{d}}\left((-\Delta)\left(I-\mu^{2} \Delta\right)^{-1} u\right)(\boldsymbol{x}) v(\boldsymbol{x}) \mathrm{d} \boldsymbol{x} \mathrm{d} \mu,
$$

for any $u, v \in H_{c}^{s}(\Omega)$.

\subsubsection{Extension representations}

An extension representation of the fractional Laplacian was introduced in Molčanov and Ostrovskiı̌ (1969) and popularized by Caffarelli and Silvestre (2007), who showed that the fractional Poisson problem (3.5) posed on $\mathbb{R}^{d}$ can be recast as a Neumann-to-Dirichlet mapping over the extended domain $\mathbb{R}^{d} \times[0, \infty)$, that is, we have

$$
\begin{cases}-\nabla \cdot z^{\beta} \nabla U(\boldsymbol{x}, z)=0 & \text { for all }(\boldsymbol{x}, z) \in \mathbb{R}^{d} \times[0, \infty), \\ \frac{\partial U}{\partial \nu^{\beta}}(\boldsymbol{x})=2^{1-2 s} \frac{\Gamma(1-s)}{\Gamma(s)} f(\boldsymbol{x}) & \text { for all } \boldsymbol{x} \in \mathbb{R}^{d}\end{cases}
$$


where $\beta=1-2 s$ and

$$
\frac{\partial U}{\partial \nu^{\beta}}(\boldsymbol{x})=-\lim _{z \rightarrow 0^{+}} z^{\beta} \frac{\partial U}{\partial z}(\boldsymbol{x}, z),
$$

with the solution to (3.5) recovered by taking the trace of $U$ on $\mathbb{R}^{d}$.

The spectral fractional Laplacian can be recovered, by restricting the extension domain from $\mathbb{R}^{d} \times[0, \infty)$ to $\Omega \times[0, \infty)$ and imposing a homogeneous Dirichlet boundary condition on the lateral surface (Stinga and Torrea 2010).

The apparent advantage of the extension problem is that the nonlocal problem is replaced with a classical, integer-order local problem. This comes at the price of having to deal with a singular weight function and an additional spatial dimension.

\subsubsection{Regularity of solutions}

In the classical integer-order case, smoother domains and smoother righthand sides result in smoother solutions of the Poisson problem; see e.g. Taylor (1996). A lifting property of this type does not hold for the fractionalorder Poisson problems (3.5) and (3.14). The typical solution behaviour of (3.5) close to the boundary can be characterized (see Ros-Oton and Serra 2014) as

$$
u(\boldsymbol{x}) \approx \operatorname{dist}(\boldsymbol{x}, \partial \Omega)^{s} .
$$

The Sobolev regularity of the solution of (3.5) was studied in Acosta, Bersetche and Borthagaray (2017) as a special case of a result in Grubb (2015a). Suppose that $\partial \Omega \in C^{\infty}$ and $f \in H^{r}(\Omega)$ for $r \geq-s$ and let $u \in H_{c}^{s}(\Omega)$ denote the solution of the fractional Poisson problem (3.7). Then we obtain the regularity estimate

$$
u \in \begin{cases}H^{2 s+r}(\Omega) & \text { if } 0<s+r<1 / 2, \\ H^{s+1 / 2-\varepsilon}(\Omega) \text { for all } \varepsilon>0 & \text { if } 1 / 2 \leq s+r .\end{cases}
$$

This result is extended to the case of non-homogeneous volume condition in Acosta et al. (2019). Additional regularity results with respect to $s$ and, for the truncated fractional Laplacian, with respect to $\delta$, are derived in Burkovska and Gunzburger (2019b).

The solution of the spectral fractional Poisson problem (3.14) displays different properties. Its behaviour close to the boundary is given by (see Caffarelli and Stinga 2016)

$$
u(\boldsymbol{x}) \approx \operatorname{dist}(\boldsymbol{x}, \partial \Omega)^{\min \{2 s, 1\}}, \quad s \neq 1 / 2 .
$$

Grubb (2016) showed that if $f \in H_{c}^{r}(\Omega), r \geq-s$, then the weak solution of the spectral fractional Poisson problem (3.14) satisfies $u \in H_{c}^{r+2 s}(\Omega)$. 
More detailed regularity results for the spectral fractional Laplacian can be found in Grubb (2016).

\subsubsection{Analytic solutions in one and two dimensions}

Closed-form solutions of the fractional Poisson problem (3.5) posed on the unit ball are available; see Dyda, Kuznetsov and Kwaśnicki (2017b) for detailed derivations. These solutions are useful to have in hand, e.g. for verifying numerical results.

In $d=1$ dimension, for the source term

$$
f_{k, 0}^{1 D}=2^{2 s} \Gamma(1+s)^{2}\left(\begin{array}{c}
s+k-1 / 2 \\
s
\end{array}\right)\left(\begin{array}{c}
s+k \\
s
\end{array}\right) P_{k}^{(s,-1 / 2)}\left(2 x^{2}-1\right)
$$

with $k \geq 0$, the solution is given by

$$
u_{k, 0}^{1 D}=P_{k}^{(s,-1 / 2)}\left(2 x^{2}-1\right)\left(1-x^{2}\right)_{+}^{s},
$$

where

$$
\left(\begin{array}{l}
a \\
b
\end{array}\right)=\frac{\Gamma(a+1)}{\Gamma(b+1) \Gamma(a-b+1)}
$$

denotes a generalized binomial coefficient, $P_{k}^{(a, b)}$ denote the Jacobi polynomials, and $a_{+}=\max \{0, a\}$. Moreover, for

$$
f_{k, 1}^{1 D}=2^{2 s} \Gamma(1+s)^{2}\left(\begin{array}{c}
s+k+1 / 2 \\
s
\end{array}\right)\left(\begin{array}{c}
s+k \\
s
\end{array}\right) x P_{k}^{(s, 1 / 2)}\left(2 x^{2}-1\right)
$$

with $k \geq 0$, the solution is given by

$$
u_{k, 1}^{1 D}=x P_{k}^{(s, 1 / 2)}\left(2 x^{2}-1\right)\left(1-x^{2}\right)_{+}^{s} .
$$

Turning to $d=2$ dimensions, for

$$
f_{k, \ell}^{2 D}=2^{2 s} \Gamma(1+s)^{2}\left(\begin{array}{c}
s+k+\ell \\
s
\end{array}\right)\left(\begin{array}{c}
s+k \\
s
\end{array}\right) r^{\ell} \cos (\ell \varphi) P_{k}^{(s, \ell)}\left(2 r^{2}-1\right)
$$

with $\ell, k \geq 0$ and $(r, \varphi)$ denoting polar coordinates, the solution is given by

$$
u_{k, \ell}^{2 D}=r^{\ell} \cos (\ell \varphi) P_{k}^{(s, \ell)}\left(2 r^{2}-1\right)\left(1-r^{2}\right)_{+}^{s} .
$$

Two example solutions are shown in Figure 3.1. Observe that the solutions contain a term that behaves like $\delta(\boldsymbol{x})^{s}$, where $\delta(\boldsymbol{x})$ is the distance from $\boldsymbol{x} \in \Omega$ to the boundary.

\subsection{Inhomogeneous constitutive functions in fractional models}

So far in this section we have only considered the fractional Laplacian and its variants, which is analogous to considering local diffusion problems having a diffusivity that is not only constant but equal to one. Of course, in the 

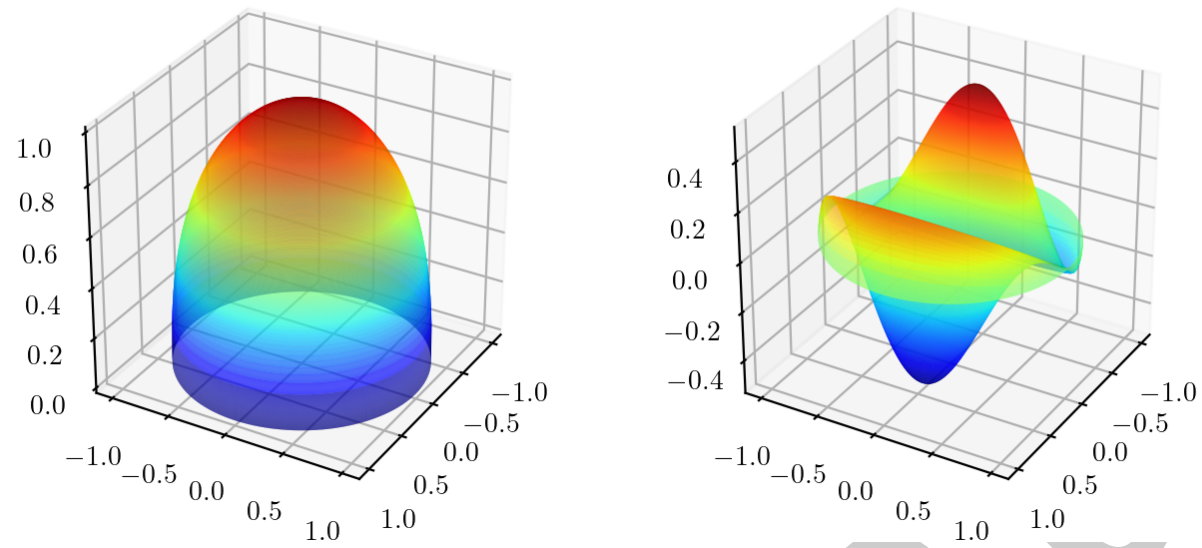

Figure 3.1. Analytic solutions $u_{0,0}^{2 D}$ for $s=0.4$ and $u_{1,1}^{2 D}$ for $s=0.6$. The behaviour (3.17) close to the boundary is apparent.

local case, it is an easy matter to treat inhomogeneous diffusivities, that is, we merely replace $\Delta u$ with $\nabla \cdot(\boldsymbol{D} \nabla u)$, where $\boldsymbol{D}(\boldsymbol{x})$ generally denotes an inhomogeneous constitutive tensor. Using the nonlocal vector calculus, it is almost as easy to define integral fractional models in which the constitutive properties of the media considered are inhomogeneous even if the nonlocal constitutive function is a tensor. Here we only consider generalizations of the integral fractional Laplacian (3.1); generalizations of the other integral operators discussed in this section follow immediately from that for (3.1).

The inhomogeneous fractional Laplacian is simply defined by choosing the kernel in (3.1) to now be

$$
\gamma_{s}(\boldsymbol{x}, \boldsymbol{y})=C_{d, s} \frac{\theta_{s}(\boldsymbol{x}, \boldsymbol{y})}{|\boldsymbol{y}-\boldsymbol{x}|^{d+2 s}} \quad \text { for all } \boldsymbol{x}, \boldsymbol{y} \in \mathbb{R}^{d},
$$

where

$$
\theta_{s}(\boldsymbol{x}, \boldsymbol{y}):=\frac{\boldsymbol{y}-\boldsymbol{x}}{|\boldsymbol{y}-\boldsymbol{x}|} \cdot\left(\Theta_{s}(\boldsymbol{x}, \boldsymbol{y}) \frac{\boldsymbol{y}-\boldsymbol{x}}{|\boldsymbol{y}-\boldsymbol{x}|}\right)
$$

with $\boldsymbol{\Theta}_{s}(\boldsymbol{x}, \boldsymbol{y})$ denoting a constitutive tensor. Thus we have defined the inhomogeneous integral fractional Poisson operator

$$
(-\Delta)_{\mathbf{\Theta}_{s}}^{s} u(\boldsymbol{x}):=\int_{\mathbb{R}^{d}} C_{d, s} \theta_{s}(\boldsymbol{x}, \boldsymbol{y}) \frac{u(\boldsymbol{x})-u(\boldsymbol{y})}{|\boldsymbol{y}-\boldsymbol{x}|^{d+2 s}} \mathrm{~d} \boldsymbol{y} \quad \text { for all } \boldsymbol{x} \in \mathbb{R}^{d} .
$$

Note that we could have arrived at (3.20) by defining

$$
(-\Delta)_{\Theta_{s}}^{s} u(\boldsymbol{x}):=\frac{1}{2} \mathcal{D}_{s}\left(\boldsymbol{\Theta}_{s} \mathcal{D}_{s}^{*} u\right),
$$


where $\mathcal{D}_{s}$ and $\mathcal{D}_{s}^{*}$ are defined by (1.13) and (1.15), respectively, with

$$
\boldsymbol{\alpha}_{s}(\boldsymbol{x}, \boldsymbol{y})=\frac{\boldsymbol{y}-\boldsymbol{x}}{|\boldsymbol{y}-\boldsymbol{x}|} \sqrt{\frac{C_{d, s}}{|\boldsymbol{y}-\boldsymbol{x}|^{d+2 s}}} .
$$

If $\boldsymbol{\Theta}_{s}(\boldsymbol{x}, \boldsymbol{y})=\boldsymbol{\Theta}_{s}(|\boldsymbol{y}-\boldsymbol{x}|)$, i.e. $\boldsymbol{\Theta}_{s}$ is a radial function, then (3.1) is a model for a homogeneous medium, that is, in this case, (3.1) is a fractional analogue of the PDE $-\nabla \cdot(D \nabla u)=f$ in which $\boldsymbol{D}$ is a constant tensor. If in addition $\boldsymbol{\Theta}_{s}$ is a scalar tensor, then (3.1) is a fractional analogue of the $\mathrm{PDE}-\kappa \Delta u=f$ in which $\kappa$ is a constant. Thus, to obtain an inhomogeneous integral fractional model, $\boldsymbol{\Theta}_{s}(\boldsymbol{x}, \boldsymbol{y})$ cannot be a radial function. See Remark 2.3.

\section{PART TWO}

\section{Numerical methods for nonlocal and fractional models}

In this part we consider the approximation, via finite element, finite difference and spectral methods, of solutions of both weak and strong formulations of both nonlocal and fractional diffusion models. In so doing, we encounter, as we did in Part 1 , problems posed on $\mathbb{R}^{d}$ or on bounded domains $\Omega \subset \mathbb{R}^{d}$, and also encounter problems for which the horizon $\delta=\infty$ or $\operatorname{diam}(\Omega)<\delta<\infty$ or $\delta<\operatorname{diam}(\Omega)$.

\section{Introductory remarks}

In most cases we consider, two parameters will appear in the design of discretization algorithms, namely the horizon $\delta$ and a discretization parameter such as a grid size parameter $h$ for finite element and finite difference methods or the dimension $N$ of a basis for spectral methods. The limiting behaviours of continuous and discretized models are thus of interest. Four types of limits can arise: for continuous, i.e. un-discretized, models,

- the limits $\delta \rightarrow 0$ and $\delta \rightarrow \infty$,

and for discretized problems,

- for fixed $\delta$, the limit $h \rightarrow 0$ or $N \rightarrow \infty$,

- for fixed $h$ or $N$, the limits $\delta \rightarrow 0$ and $\delta \rightarrow \infty$,

- simultaneous limits such as both $\delta \rightarrow 0$ and $h \rightarrow 0$.

For the first of these, one can find discussions of the local and global (fractional) limits of nonlocal continuum models in, for example, Mengesha and Du (2015), Tian, Du and Gunzburger (2016) and Du (2019). For the second 
of these, one must be cognizant of how the constants appearing in error estimates depend on $\delta$, whereas for the third, the same can be said for the dependence of constants on $h$ or $N$.

It turns out that for some algorithms, the order in which limits are taken matters, that is, the limits obtained are different if the limits are taken in a different order, e.g. $\delta \rightarrow 0$ and then $h \rightarrow 0$, or $h \rightarrow 0$ and then $\delta \rightarrow 0$, or if $\delta$ and $h$ are related in some way so that they simultaneously tend to zero. Presumably, it is possible that for some algorithms at least one of the limits is 'wrong' in some sense. Ideally, unless one is totally uninterested in limiting behaviours, one would prefer the way in which limits are taken not to affect the limit obtained. In the remainder of our introductory remarks, we expand on this concept.

\subsection{Asymptotically compatible schemes}

It is known in practice that to obtain consistency between nonlocal models and corresponding local PDE models, the mesh or quadrature point spacing may have to be reduced at a faster pace than the reduction of the horizon parameter (Bobaru et al. 2009, Bobaru and Duangpanya 2010, Chen and Gunzburger 2011). Otherwise there could potentially be complications, most notably inconsistent limiting solutions when the horizon parameter is coupled proportionally to the discretization parameter (Tian 2017, Tian and Du 2013, Tian and Du 2014, Tian and Du 2020). Asymptotically compatible (AC) schemes, motivated by the findings in Tian and Du (2013) and formally introduced in Tian and Du (2014), are numerical discretizations of nonlocal models that converge to nonlocal continuum models for a fixed horizon parameter and to the local discrete schemes as the horizon vanishes for both discrete schemes with a fixed numerical resolution and for continuum models with increasing numerical resolution.

Let $h>0$ denote a grid size parameter (or particle spacing) and let $\delta$ denote the horizon parameter or even a more generic model parameter. Instead of $h$, we could use the dimension $N$ of a spectral basis. The implications of the AC property are illustrated in Figure 4.1. There, $u_{\delta}$ denotes the solution of the continuous nonlocal problem with $\delta>0, u_{0}$ the solution of the corresponding continuous local problem, $u_{\delta, h}$ the solution of the discretized nonlocal problem, and $u_{0, h}$ the solution of the discretized local problem. Figure 4.1 is meant to illustrate the basic property of AC schemes, namely that for such schemes it does not matter in which order the limits are taken, or even if $\delta$ and $h$ are related in some way so that they simultaneously tend to zero. Note that figures similar to Figure 4.1 can be drawn for $N \rightarrow \infty$ and/or $\delta \rightarrow \infty$.

As seen from detailed studies given in Tian and Du (2013), some popular discretization schemes for nonlocal peridynamics fail to be AC. In particular, 


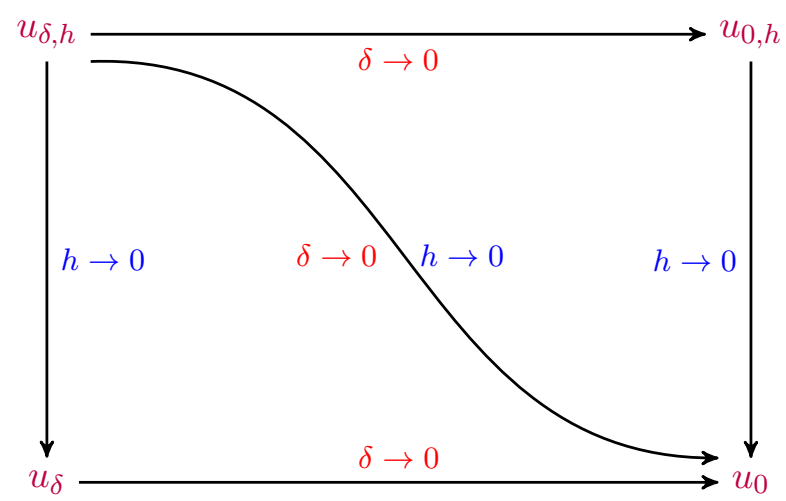

Figure 4.1. An illustrative diagram for AC schemes.

if $\delta$ is taken to be proportional to $h$, then as $h \rightarrow 0$, piecewise constant conforming finite element solutions actually converge to the incorrect limit, similarly to those based on simple Riemann sum quadrature approximations to nonlocal operators. Similar discussions can be found in Bobaru et al. (2009) and Chen and Gunzburger (2011) for diffusion models and Seleson, Du and Parks (2016) for systems of peridynamic equations.

Asymptotically compatible (AC) schemes, such as conforming Galerkintype approximations of weak forms (Chen and Gunzburger 2011, Tian and Du 2014, Xu, Gunzburger, Burkardt and Du 2016b), nonconforming discontinuous Galerkin approximations (Du, Ju, Lu and Tian 2020a, Du, Ju and Lu 2019b, Du and Yin 2019) or collocation- or quadrature-based approximations of strong forms (Du and Tian 2014, Seleson et al. 2016, Du et al. 2019c, Zhang, Gunzburger and Ju 2016a, Zhang, Gunzburger and Ju 2016b), offer the potential to solve for approximations of a model of interest with different choices of parameters to gain efficiency and to avoid the pitfall of reaching inconsistent limits.

\section{Finite element methods for nonlocal models}

Given weak formulations of nonlocal models, it is natural to consider finite element approximations (Chen and Gunzburger 2011, Tian and Du 2013, Tian and Du 2014, Xu, Gunzburger and Burkardt 2016a, Xu et al. 2016b, Tian and Du 2020, Du 2019, Jha and Lipton 2020). To derive a finite element discretization, one possible way is to follow the same recipe as that used for the local PDE setting. We assume that $\Omega \subset \mathbb{R}^{d}$ is a polytope, so the first step is to construct a regular subdivision of $\Omega \cup \Omega_{\mathcal{I}_{\delta}}$ into finite elements, e.g. triangles or quadrilaterals for $d=2$. Based on the grid, we then define a finite element space $V^{h}\left(\Omega \cup \Omega_{\mathcal{I}_{\delta}}\right)$, usually consisting of piecewise polynomial functions with respect to the grid, and then choose a 
basis for that space containing functions whose support extends over a few contiguous elements. We do not dwell on the construction of finite element spaces because it is the same as that for the local PDE setting (Brenner and Scott 1994, Ciarlet 2002, Ern and Guermond 2004), except perhaps that it is prudent to have the grid contain a regular subdivision of $\Omega$ itself so that the boundary of $\Omega$ is subdivided into $(d-1)$-dimensional faces. For conforming finite element methods, that is, the finite element space $V^{h}\left(\Omega \cup \Omega_{\mathcal{I}_{\delta}}\right)$ is a subset of the energy space $V\left(\Omega \cup \Omega_{\mathcal{I}_{\delta}}\right)$ for the continuous problem, we define the constrained finite element space

$$
V_{c}^{h}\left(\Omega \cup \Omega_{\mathcal{I}_{\delta}}\right)=\left\{v(\boldsymbol{x}) \in V^{h}\left(\Omega \cup \Omega_{\mathcal{I}_{\delta}}\right): v(\boldsymbol{x})=0 \text { for all } \boldsymbol{x} \in \Omega_{\mathcal{I}_{\delta}}\right\} .
$$

Note that $V_{c}^{h}\left(\Omega \cup \Omega_{\mathcal{I}_{\delta}}\right) \subset V_{c}\left(\Omega \cup \Omega_{\mathcal{I}_{\delta}}\right)$. We let $g_{h}(\boldsymbol{x})$ denote an approximation to $g(\boldsymbol{x})$, for example, $g_{h}(\boldsymbol{x})$ could be the interpolant of $g(\boldsymbol{x})$ in $V_{c}^{h}\left(\Omega \cup \Omega_{\mathcal{I}_{\delta}}\right)$ restricted to $\Omega_{\mathcal{I}_{\delta}}$, or, if $g(\boldsymbol{x})$ is not smooth enough to have pointwise values, a least-squares approximation could be used instead. A conforming finite element approximation $u_{h}(\boldsymbol{x}) \in V^{h}\left(\Omega \cup \Omega_{\mathcal{I}_{\delta}}\right)$ of the solution of $u(\boldsymbol{x}) \in V\left(\Omega \cup \Omega_{\mathcal{I}_{\delta}}\right)$ of $(2.9)$ is then determined by solving the following problem:

Given $\gamma_{\delta}(\boldsymbol{x}, \boldsymbol{y})$ defined in (1.17) and given $f(\boldsymbol{x}) \in V_{d}(\Omega)$, find $u_{h}(\boldsymbol{x}) \in V^{h}\left(\Omega \cup \Omega_{\mathcal{I}_{\delta}}\right)$ such that

$$
\begin{cases}\mathcal{A}_{\delta}\left(u_{h}, v_{h}\right)=\left\langle f, v_{h}\right\rangle & \text { for all } v \in V_{c}^{h}\left(\Omega \cup \Omega_{\mathcal{I}_{\delta}}\right), \\ u_{h}(\boldsymbol{x})=g_{h}(\boldsymbol{x}) & \text { for all } \boldsymbol{x} \in \Omega_{\mathcal{I}_{\delta}},\end{cases}
$$

where $g_{h}(\boldsymbol{x})$ is defined as discussed above. Because the bilinear form $\mathcal{A}_{\delta}(\cdot, \cdot)$ is continuous and coercive with respect to $V_{c}\left(\Omega \cup \Omega_{\mathcal{I}_{\delta}}\right)$, it is likewise continuous and coercive with respect to $V_{c}^{h}\left(\Omega \cup \Omega_{\mathcal{I}_{\delta}}\right)$, so the well-posedness of problem (5.1) follows immediately from the Lax-Milgram theorem. For problems involving singular nonlocal interaction kernels such as the fractional type given in Table 2.1, special numerical quadrature is needed for the evaluation of stiffness matrices. We refer to Section 6.1 for related discussions.

Remark 5.1 (DG methods in the nonlocal setting). One of the major differences between finite element methods for local PDEs and nonlocal models occurs in the use of discontinuous Galerkin (DG) methods. For elliptic PDEs, DG methods are not conforming, that is, a finite element space containing functions with jump discontinuities cannot be a subspace of the space $H^{1}(\Omega)$ in which the PDE is well-posed. As a result, the use of DG methods requires an accounting for fluxes across element faces; otherwise, elements would be uncoupled. On the other hand, for some kernel choices, finite element functions with jump discontinuities belong to the energy space $V\left(\Omega \cup \Omega_{\mathcal{I}_{\delta}}\right)$ in which the nonlocal problem is well-posed. This is the case for kernels that are both radial and integrable and for the fractional kernel 
with $s<1 / 2$, as listed in Table 2.1. For such kernels DG methods are conforming, so that, at least for $\delta>h$, no explicit accounting for fluxes across element faces is needed. The coupling between elements is taken care of by nonlocality, that is, even though the basis functions live only on single elements, any specific element is coupled to all other elements that contain points that are within a distance $\delta$ to some point in the specific element. For more singular kernels where DG methods are not conforming, e.g. the fractional kernels with $s>1 / 2$, we refer to the discussions in Section 5.2.

\subsection{Asymptotically compatible conforming finite element methods for nonlocal diffusion}

To delve deeper into finite element methods for nonlocal diffusion with a finite range of interactions, we first specialize to a more specific setting for which the local diffusion limit has been rigorously established. Consider the model given by (2.9) with $g=0$. We also choose the kernel $\gamma_{\delta}(\boldsymbol{x}, \boldsymbol{y})$ as a rescaled translation-invariant kernel, that is,

$$
\gamma_{\delta}(\boldsymbol{x}, \boldsymbol{y})=\frac{1}{\delta^{d+2}} \gamma\left(\frac{|\boldsymbol{y}-\boldsymbol{x}|}{\delta}\right)
$$

for $\delta \in(0,1]$ and some kernel $\gamma(|\cdot|)$ that is assumed to be radial, nonnegative, compactly supported in $B_{1}(\mathbf{0})$ (the unit ball centred at the origin), and has a bounded second-order moment, that is,

$$
\widehat{\gamma}(|\boldsymbol{\xi}|)=|\boldsymbol{\xi}|^{2} \gamma(|\boldsymbol{\xi}|) \in L_{\mathrm{loc}}^{1}\left(\mathbb{R}^{d}\right) \quad \text { and } \quad \int_{B_{1}(\mathbf{0})} \widehat{\gamma}(|\boldsymbol{\xi}|) \mathrm{d} \boldsymbol{\xi}=d .
$$

For kernels satisfying these conditions, we refer to the discussions in Section 2.2. For such kernels, the local $\delta \rightarrow 0$ limit becomes the homogeneous Dirichlet boundary value problem of the Poisson equation. Let us denote the energy space $V_{c, \delta}\left(\Omega \cup \Omega_{\mathcal{I}_{\delta}}\right)=V_{c}\left(\Omega \cup \Omega_{\mathcal{I}_{\delta}}\right)$ as defined in (2.4) to highlight the dependence on $\delta$.

Now, for any fixed $\delta \in(0,1]$, we introduce conforming finite element spaces $\left\{V_{\delta, h}\right\} \subset V_{c, \delta}\left(\Omega \cup \Omega_{\mathcal{I}_{\delta}}\right)$ associated with the triangulation $\tau_{h}=\{K\}$ of the domain $\Omega \cup \Omega_{\mathcal{I}_{\delta}}$ (or $\Omega \cup \Omega_{\mathcal{I}_{1}}$ that contains the domain $\Omega \cup \Omega_{\mathcal{I}_{\delta}}$ for any $\delta<1)$. We set

$$
V_{\delta, h}:=\left\{v \in V_{c, \delta}\left(\Omega \cup \Omega_{\mathcal{I}_{\delta}}\right):\left.v\right|_{K} \in P_{p}(K) \text { for all } K \in \tau_{h}\right\},
$$

where $P_{p}(K)$ denotes the space of polynomials on $K \in \tau_{h}$ of degree less than or equal to $p$. Again, for different $\delta$, in order to have the finite element functions defined on a common spatial domain, we also assume, as for $V_{c, \delta}\left(\Omega \cup \Omega_{\mathcal{I}_{\delta}}\right)$, that any element in $V_{\delta, h}$ vanishes outside $\Omega$.

As $h \rightarrow 0$, we assume that $\left\{V_{\delta, h}\right\}$ is dense in $V_{c, \delta}\left(\Omega \cup \Omega_{\mathcal{I}_{\delta}}\right)$, that is, for any $v \in V_{c, \delta}\left(\Omega \cup \Omega_{\mathcal{I}_{\delta}}\right)$, there exists a sequence $\left\{v_{h} \in V_{\delta, h}\right\}$ such that, for a 
given $\delta>0$,

$$
\left\|v_{h}-v\right\|_{V_{c, \delta}\left(\Omega \cup \Omega_{\mathcal{I}_{\delta}}\right)} \rightarrow 0 \quad \text { as } h \rightarrow 0 .
$$

These properties are easily satisfied by standard finite element spaces.

The Galerkin approximation is defined by replacing $V_{c, \delta}\left(\Omega \cup \Omega_{\mathcal{I}_{\delta}}\right)$ with $V_{\delta, h}$ in (2.9):

Find $u_{h, \delta} \in V_{\delta, h}$ such that $\mathcal{A}_{\delta}\left(u_{h, \delta}, v_{h}\right)=\left\langle f, v_{h}\right\rangle$ for all $v_{h} \in V_{\delta, h}$.

The analysis of Galerkin approximations of parametrized nonlocal variational problems can be formulated within the general framework of AC schemes (Tian and Du 2014). Other than the necessary properties of functions and operators that guarantee $u_{\delta} \rightarrow u_{0}$ as $\delta \rightarrow 0$, where $u_{0}$ denotes the solution of the corresponding PDE, and standard density properties such as (5.4), one of the key ingredients for the asymptotic compatibility of the scheme is the asymptotic density property of the finite element space. Rather than a general definition, we provide a specific instance, adapted to the models studied here, as follows. A family of finite-dimensional spaces $\left\{V_{\delta, h} \subset V_{c, \delta}\left(\Omega \cup \Omega_{\mathcal{I}_{\delta}}\right), \delta \in(0,1), h \in\left(0, h_{0}\right]\right\}$ is asymptotically dense in $H_{0}^{1}(\Omega)$, if, for all $v \in H_{0}^{1}(\Omega)$, there exists a sequence $\left\{v_{n} \in V_{\delta_{n}, h_{n}}\right\}_{h_{n} \rightarrow 0, \delta_{n} \rightarrow 0}$ as $n \rightarrow \infty$ such that

$$
\left\|v-v_{n}\right\|_{H^{1}(\Omega)} \rightarrow 0 \quad \text { as } n \rightarrow \infty .
$$

Tian and Du (2014) have shown rigorously that, for scalar nonlocal diffusion equations such as (2.9), all conforming Galerkin approximations of the nonlocal models containing continuous piecewise linear functions are automatically AC in any space dimension. This means that they can recover the correct local limit as long as both $\delta$ and $h$ are decreasing, even if the nonlocal parameter $\delta$ is reduced at a much faster pace than the mesh spacing $h$. Even though the analysis of the above AC property is highly technical, an intuitive explanation is that even with $h$ larger than $\delta$, the nonlocal features that ensure the correct local limit are still encoded in the stiffness matrices thanks to higher-order (than constant) basis functions. In fact, even though, for the class of kernels we are considering, discontinuous piecewise constant finite element spaces are conforming, they do not generally result in AC approximations. This was first noticed numerically by Chen and Gunzburger (2011).

For general nonlocal systems in any dimension, Tian and Du (2014, 2020) have shown that as long as the condition $h=o(\delta)$ is met as $\delta \rightarrow 0$, then we obtain the correct local limit even for discontinuous piecewise constant finite element approximations when they are of the conforming type. Practically speaking, this implies that a mild growth of the bandwidth in the finite element stiffness matrix is needed as the mesh is refined in order to recover the correct local limit for piecewise constant finite element schemes. In fact, 
Tian and $\mathrm{Du}(2013,2015)$ have shown that if a constant bandwidth is kept as the mesh is refined, the approximations may converge to an incorrect local limit.

Naturally, schemes using higher-order basis functions tend to provide higher-order accuracy in the nonlocal setting as well, should the solutions enjoy sufficient regularity. At the moment, the theory on AC schemes does not offer any estimate of the order of convergence with respect to different couplings of $h$ and $\delta$. Preliminary numerical experiments in Tian and Du (2014) offer some insight about the balance of modelling and discretization errors, but additional theoretical analyses need to be carried out, except for the case of Fourier spectral approximations of nonlocal models with periodic boundary conditions, for which precise error estimates can be found in $\mathrm{Du}$ and Yang $(2016,2017)$ and Slevinsky, Montanelli and Du (2018).

\subsection{Nonconforming and DG FEMs for nonlocal models with sufficiently singular kernels}

The framework of AC schemes is very general. For example, the theory also guided the development of nonconforming discontinuous Galerkin approximations (Tian and Du 2015) for nonlocal diffusion and nonlocal peridynamic models with sufficiently singular interaction kernels. Consider the scalar model given by (2.9) with $g=0$. If the nonlocal interaction kernel satisfies

$$
\int_{|\boldsymbol{x}|<\epsilon}|\boldsymbol{x}| \gamma_{\delta}(|\boldsymbol{x}|) \mathrm{d} \boldsymbol{x}=\infty \text { for all } \epsilon \in\left(0, \epsilon_{0}\right],
$$

then it may be the case that discontinuous finite element solutions do not belong to the associated energy space and alternative formulations have to be developed. For technical reasons, Tian and Du (2015) also assumed that

$$
\lim _{\epsilon \rightarrow 0} \epsilon^{2}\left(\int_{|\boldsymbol{x}|<\epsilon}|\boldsymbol{x}|^{2} \gamma_{\delta}(|\boldsymbol{x}|) \mathrm{d} \boldsymbol{x}\right)^{-1}=0 .
$$

Note that for kernels $\gamma_{\delta}(r)$ that behave like $1 / r^{d+2 s}$ as $r \rightarrow 0$ with $s \in$ $(1 / 2,1)$, conditions (5.7) and (5.8) are satisfied.

Tian and Du (2015) introduced a nonconforming DG scheme based on the removal of the singularity in the nonlocal interaction kernel in a sufficiently small neighbourhood of the origin parametrized by a cut-off level $n$. In other words, $\gamma_{\delta}(r)$ in $(2.9)$ is replaced with

$$
\gamma_{\delta}^{n}(r):= \begin{cases}\gamma_{\delta}(r) & \text { if } \gamma_{\delta}(r) \leq n \\ n & \text { if } \gamma_{\delta}(r)>n\end{cases}
$$

For nonlocal problems with the regularized kernel $\gamma_{\delta}^{n}(r)$, discontinuous element spaces, such as conventional discontinuous finite element spaces, can 
be used as conforming Galerkin approximations, leading to a discrete solution $u_{h, \delta, n}$. Naturally, there are other ways to define the cut-off. The essential requirement is that the resulting modified kernel is both radial and integrable (for a given $n$ ) and it converges pointwise to the original kernel.

The goal is then to demonstrate that $u_{h, \delta, n}$ converges to the solution $u_{\delta}$ of the original continuous nonlocal model with a singular kernel as $n \rightarrow \infty$ and $h \rightarrow 0$. The convergence theory can be established by generalizing the relevant compactness results given in Bourgain, Brezis and Mironescu (2001) as $n \rightarrow \infty$ for a given $\delta>0$. Indeed, the following generalization is made in Tian and $\mathrm{Du}(2015)$.

Compactness results. Given the kernels $\gamma_{\delta}^{n}$ and $\gamma_{\delta}$ and the corresponding energy spaces $V_{c, \delta}^{n}\left(\Omega \cup \Omega_{\mathcal{I}_{\delta}}\right)$ and $V_{c, \delta}\left(\Omega \cup \Omega_{\mathcal{I}_{\delta}}\right)$, assume that the energy norms of $\left\{v_{n} \in V_{c, \delta}^{n}\left(\Omega \cup \Omega_{\mathcal{I}_{\delta}}\right)\right\}$ have the uniform bound

$$
\operatorname{supp}_{n} \int_{\Omega \cup \Omega_{\mathcal{I}_{\delta}}} \int_{\Omega \cup \Omega_{\mathcal{I}_{\delta}}} \gamma_{\delta}^{n}(|\boldsymbol{y}-\boldsymbol{x}|)\left(v_{n}(\boldsymbol{y})-v_{n}(\boldsymbol{x})\right)^{2} \mathrm{~d} \boldsymbol{y} \mathrm{d} \boldsymbol{x} \leq C_{0} .
$$

Then $\left\{v_{n}\right\}$ is relatively compact in $L^{2}\left(\Omega \cup \Omega_{\mathcal{I}_{\delta}}\right)$ and any limit function $v$ belongs to $V_{c, \delta}\left(\Omega \cup \Omega_{\mathcal{I}_{\delta}}\right)$ with

$$
\int_{\Omega \cup \Omega_{\mathcal{I}_{\delta}}} \int_{\Omega \cup \Omega_{\mathcal{I}_{\delta}}} \gamma_{\delta}(|\boldsymbol{y}-\boldsymbol{x}|)(v(\boldsymbol{y})-v(\boldsymbol{x}))^{2} \mathrm{~d} \boldsymbol{x} \mathrm{d} \boldsymbol{y} \leq C_{0} .
$$

The classical Bourgain-Brezis-Mironescu compactness result established in Bourgain et al. (2001) can be seen as the local limit of the new compactness result for nonlocal spaces in Tian and Du (2015) with a finite $\delta$. By applying the framework of asymptotically compatible discretization (Tian and Du 2014, Tian and Du 2020), reviewed earlier with respect to the mesh parameter $h$ and the cut-off level $n$, we can obtain the convergence of $u_{h, \delta, n}$ to $u_{\delta}$ unconditionally as $n \rightarrow \infty$ and $h \rightarrow 0$ if the underlying finite element space contains the continuous piecewise linear finite element space. Moreover, in this case, if $n \rightarrow \infty$, we expect that for any given $\delta$ and $h$, $u_{h, \delta, n} \rightarrow u_{h, \delta}$ as $n \rightarrow \infty$, where $u_{h, \delta}$ denotes the conforming finite element approximation of $u_{\delta}$ in the space $V_{\delta, h} \cap V_{c, \delta}\left(\Omega \cup \Omega_{\mathcal{I}_{\delta}}\right)$.

If piecewise constant finite elements are used, a conditional convergence theorem has been established in Tian and Du (2015), provided the definition of the cut-off is suitably modified. For instance, consider a kernel of the type

$$
\frac{\gamma_{\star}}{|\boldsymbol{y}-\boldsymbol{x}|^{d+2 s}} \leq \gamma_{\delta}(|\boldsymbol{x}-\boldsymbol{y}|) \leq \frac{\gamma^{\star}}{|\boldsymbol{y}-\boldsymbol{x}|^{d+2 s}} \quad \text { for } \boldsymbol{x}, \boldsymbol{y} \in \Omega \cup \Omega_{\mathcal{I}_{\delta}},
$$

for some $s \in(1 / 2,1)$ and positive constants $\gamma_{\star}$ and $\gamma^{\star}$. Then one may let

$$
\gamma_{\delta}^{n}(r):= \begin{cases}\gamma_{\delta}(r) & \text { for } r \geq 1 / n \\ \gamma_{\delta}(1 / n) & \text { for } 0<r<1 / n .\end{cases}
$$


For the approximate solution $u_{h, \delta, n}$ defined as the Galerkin approximation to the nonlocal problem with kernel $\gamma_{\delta}^{n}$ using piecewise constants, we have that $\left\|u_{h, \delta, n}-u_{\delta}\right\|_{L^{2}} \rightarrow 0$ if $h=o(1 / n)$ as $n \rightarrow \infty$.

We note that the nonconforming approximations discussed in Tian and $\mathrm{Du}$ (2015) in the local limit do not yield a standard nonconforming finite element approximation or a DG approximation to the local problem. One may construct other alternative formulations that can give rise to the conventional nonconforming and DG discretization of local PDEs; see e.g. Du et al. $(2020 a)$ for a study based on the DG with penalty formulation.

\subsection{Adaptive mesh refinement for nonlocal models}

Due to reduced sparsity, for instance, nonlocal models generally incur greater computational costs than their local PDE-based counterparts. Thus, designing effective adaptive methodologies is important, and it is an area worthy of attention. So far the work has been limited to nonlocal models with a finite range of interactions.

$\mathrm{Du}$, Ju, Tian and Zhou (2013b) provided an a posteriori error analysis of conforming finite element methods for solving linear nonlocal diffusion and peridynamic models. The approach adopted is a residual-type error estimator in the $L^{2}$-norm, e.g. of the form $\left\|-\mathcal{L}_{\delta}\left(u_{\delta, h}\right)-f\right\|_{L^{2}}$, which remains well-defined and can be easily computed for kernel functions that are both radial and integrable from element-wise contributions without worrying about flux jumps across element boundaries. This is in sharp contrast to the case of second-order elliptic PDEs. The theory of a posteriori error analysis has been rigorously derived for nonlocal volume-constrained problems associated with scalar equations. The reliability and efficiency of the estimators are proved, and relationships between nonlocal and classical local a posteriori error estimates are also studied.

Du, Tian and Zhao (2013c) have also developed a convergent adaptive finite element algorithm for the numerical solution of scalar nonlocal models. For problems involving certain radial but non-integrable kernel functions, the convergence of the adaptive algorithm is rigorously derived with the help of several basic ingredients, such as an upper bound on the estimator, the estimator reduction, and the orthogonality property. How these estimators and methods work in the local limit and for general time-dependent and nonlinear peridynamic models remains to be investigated.

For nonlocal problems having solutions with jump discontinuities, an adaptive finite element method is given in $\mathrm{Xu}$ et al. (2016a). There, an algorithm is developed that first detects the location of the discontinuity and then refines the grid near the discontinuity. To preserve the $h^{2}$ accuracy possible with the use of piecewise linear elements even when the exact solution contains jump discontinuities at unknown locations, the elements 


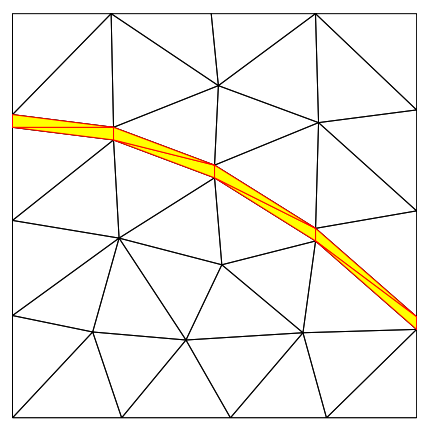

Figure 5.1. A coarse-grid illustration of a mesh resulting from the adaptive strategy discussed in this section. The elongated yellow elements surround the discontinuity in the solution.

surrounding the discontinuity should have thickness of $O\left(h^{4}\right)$ across the discontinuity. This was already observed for the one-dimensional case in Chen and Gunzburger (2011) and Xu et al. (2016b). In higher dimensions, a naive refinement strategy that results in small, well-shaped elements in the vicinity of a $(d-1)$-dimensional surface can thus result in an excessive number of degrees of freedom. The adaptive refinement strategy of $(\mathrm{Xu}$ et al. 2016a) instead results in elongated elements having thickness $O\left(h^{4}\right)$ across the discontinuity but length $O(h)$ along the discontinuity, as illustrated in Figure 5.1. The presence of elongated elements is not harmful to the error because of the anisotropic behaviour of the solution, that is, it is smooth along the discontinuity but discontinuous across the discontinuity. Whereas robust meshing algorithms for this type of anisotropic refinement and accurate predictions of solution jump discontinuities remain computationally challenging in higher space dimensions, the numerical examples in $\mathrm{Xu}$ et al. (2016a) illustrate that the adaptive strategy developed there does indeed result in $h^{2}$ convergence, as is also the case for the one-dimensional numerical results in Chen and Gunzburger (2011) and Xu et al. (2016b).

Another useful feature of the adaptive strategy developed in $\mathrm{Xu}$ et al. (2016a) is that the transition between the elongated elements along discontinuities and well-shaped elements with sides of length $O(h)$ away from the discontinuity can be abrupt, that is, there is no need to have a transition zone in which elements gradually grow in size. This is also illustrated in Figure 5.1. This feature is already present in the methods developed in Chen and Gunzburger (2011) and Xu et al. (2016b) for the one-dimensional case. The importance of this feature is that it also helps to keep down the number of degrees of freedom.

The next means of savings in the number of degrees of freedom is to use discontinuous basis functions only in the elongated elements, and continuous basis functions in elements in which the exact solution is smooth. The 
final means is to switch from the nonlocal model to the corresponding PDE model in all elements that do not interact with the elongated elements that surround the discontinuity, i.e. all elements whose vertices are at distance greater than $\delta$ from the vertices of the elongated elements.

The four degrees of freedom-saving features (i.e. elongated elements, abrupt grid transitions, the use of discontinuous basis function only in the elongated elements, and the switch to a local PDE model away from the discontinuity), result in tremendous savings in the number of degrees of freedom. In fact, the resulting number of degrees of freedom is comparable to that for a local PDE model using a regular grid of elements having sides of $O(h)$. Moreover, the relative savings become greater as the grid size is reduced and as the dimension $d$ is increased. See Xu et al. (2016a) for several numerical illustrations supporting these conclusions.

With regard to more general coupling strategies of local and nonlocal models for computational effectiveness, relevant works are discussed in a recent review on the subject (Du 2019). AC schemes have also been studied in the context of multiscale problems modelled by local-nonlocal coupling formulations with a spatially heterogeneous horizon (Du and Tian 2018, Tao, Tian and Du 2019), based on a new trace theorem for nonlocal function spaces that provides a stronger version of the classical local counterparts (Tian and Du 2017).

\subsection{Approximations of fractional models as limit of nonlocal models with a finite range of interactions}

One of the key messages we want to get across is to show that many popularly studied fractional PDEs are either specialized nonlocal models or can be treated as limiting cases.

Naturally, for fractional differential operators defined in integral form, by truncating the fractional kernel in the fractional operators to a finite range measured by the horizon parameter $\delta$, we end up with a nonlocal model parametrized by $\delta$. Hence we see from Section 3.1.1 that on the continuum level, the fractional models may then be viewed as the infinite horizon limit of nonlocal models with a finite $\delta$, after a suitable scaling of the kernel.

D'Elia and Gunzburger (2013) and Burkovska and Gunzburger (2019b) have shown that as $\delta \rightarrow \infty$, the Galerkin approximations of the nonlocal model with the parameter $\delta$ can converge to the solution of the fractional equations, provided $h$ is taken to be sufficiently small. One can actually apply the framework of the AC schemes to show that all conforming Galerkin approximations are AC in the limit $\delta \rightarrow 0$ (Tian et al. 2016). That is, the discretization of fractional equations associated with the fractional Laplacian can be viewed as the global (with an infinite nonlocal horizon parameter) limit of nonlocal models with properly scaled fractional-type 

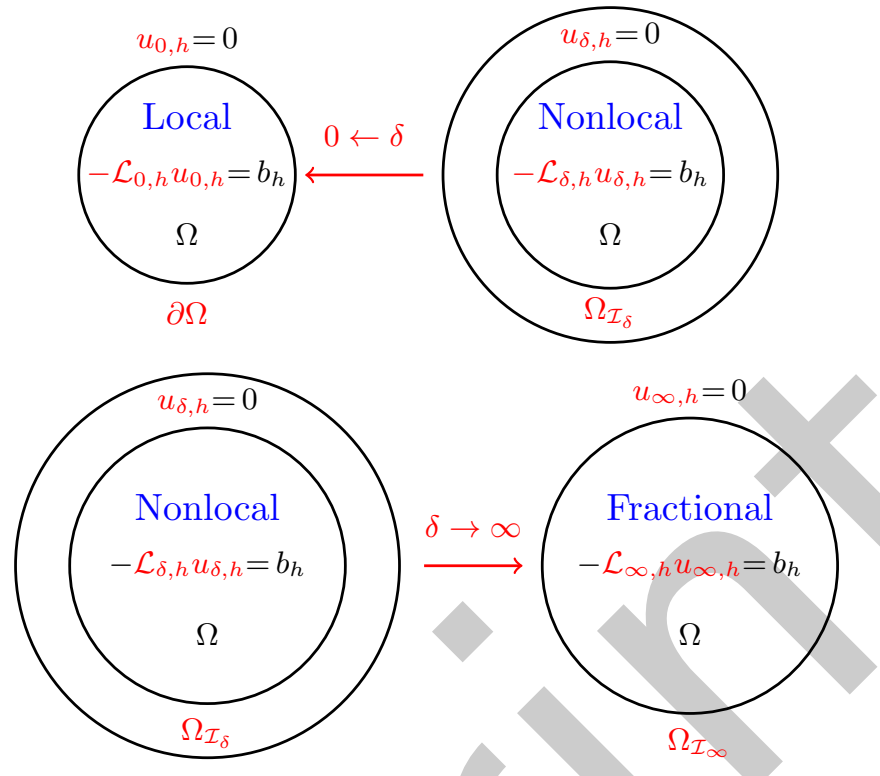

Figure 5.2. Limits of numerical solutions of nonlocal models with finite interaction radius $\delta$ as $\delta \rightarrow 0$ (local limit, top) and as $\delta \rightarrow \infty$ (fractional limit, bottom).

nonlocal interaction kernels (Tian et al. 2016). Moreover, the convergence does not require the dependence of $h$ on $\delta$.

Let us give an illustration of the work presented in Tian et al. (2016). Consider the volume-constrained problems defined for the fractional Laplacian in $\Omega$ with homogeneous Dirichlet condition in $\Omega_{\mathcal{I}_{\infty}}=\mathbb{R}^{d} \backslash \Omega$. By truncations of both the spatial domain and the range of nonlocal interactions, we may end up with a class of parametrized problems

$$
\begin{cases}-\mathcal{L}_{\delta} u(\boldsymbol{x})=-\int_{\mathbb{R}^{d}}(u(\boldsymbol{y})-u(\boldsymbol{x})) \gamma_{\delta}(|\boldsymbol{y}-\boldsymbol{x}|) \mathrm{d} \boldsymbol{y}=f & \text { for all } \boldsymbol{x} \in \Omega, \\ u=0 & \text { for all } \boldsymbol{x} \in \Omega_{\mathcal{I}_{\delta}}\end{cases}
$$

with

$$
\gamma_{\delta}(|\boldsymbol{y}-\boldsymbol{x}|)= \begin{cases}\frac{C_{d, s, \delta}}{|\boldsymbol{y}-\boldsymbol{x}|^{d+2 s}} & \boldsymbol{y} \in B_{\delta}(\boldsymbol{x}) \\ 0 & \boldsymbol{y} \in \mathbb{R}^{d} \backslash B_{\delta}(\boldsymbol{x}) .\end{cases}
$$

Here, $C_{d, s, \delta}=C_{d, s}$ is given as in (3.3) for the limiting case of $\delta \rightarrow \infty$ so that $\mathcal{L}_{\infty}=(-\Delta)^{s}$ as in (3.1). Note that in the limit of $\delta \rightarrow 0$, we take a $C_{d, s, \delta}$ to be a different scaling factor so that its local limit is $\mathcal{L}_{0}=-\Delta$.

Let $h$ denote the discretization parameter associated with Galerkin approximations of (5.13). For example, $h$ could be the mesh parameter for the 
finite element discretization or the reciprocal of the number of basis functions in spectral approximations. We can then apply the AC framework to obtain the convergence of the Galerkin approximations of (5.13) to the solution of the fractional equation as $h \rightarrow 0$ and $\delta \rightarrow \infty$ as long as the finite-dimensional approximations spaces are subspaces of the underlying energy space, that is, as long as we adopt conforming discretizations.

As pointed out in Tian et al. (2016) and Tian and Du (2020), analyses of the fractional and local limits $(\delta \rightarrow \infty$ and $\delta \rightarrow 0$ respectively) of nonlocal models parametrized by a finite $\delta$ and with fractional-type kernels demonstrate that nonlocal models are more general than their fractional and local counterparts and they also serve as a bridge between fractional and local models; see Figure 5.2 and, again, Figure 4.1 and similar diagrams given in Du (2019) and Tian and Du (2020). For AC schemes, the bridging roles of nonlocal diffusion with a finite range of nonlocal interactions, presented above at the continuum level, remain valid on the discrete level.

\section{Finite element methods for the integral fractional Laplacian}

Let $\Omega$ denote a polygon and let $\mathcal{T}_{h}$ denote a family of shape-regular and locally quasi-uniform triangulations of $\Omega$ (Ciarlet 2002, Brenner and Scott 1994, Ern and Guermond 2004). Let $\mathcal{Z}_{h}$ denote the set of vertices of $\mathcal{T}_{h}$ and let $h_{K}$ denote the diameter of the element $K \in \mathcal{T}_{h}$. Moreover, let

$$
h:=\max _{K \in \mathcal{T}_{h}} h_{K}, \quad h_{\min }:=\min _{K \in \mathcal{T}_{h}} h_{K} .
$$

Let $\varphi_{i}$ denote the usual piecewise linear Lagrange basis function associated with a node $\boldsymbol{z}_{i} \in \mathcal{Z}_{h}$, satisfying $\varphi_{i}\left(\boldsymbol{z}_{j}\right)=\delta_{i j}$ for $\boldsymbol{z}_{j} \in \mathcal{Z}_{h}$, and let $X_{h}:=$ $\operatorname{span}\left\{\varphi_{i}: \boldsymbol{z}_{i} \in \mathcal{Z}_{h}\right\}$. The finite element subspace $V_{h} \subset H_{c}^{s}(\Omega)$ is given by $V_{h}=X_{h}$ when $s<1 / 2$ and by

$$
V_{h}=\left\{v_{h} \in X_{h}: v_{h}=0 \text { on } \partial \Omega\right\}=\operatorname{span}\left\{\varphi_{i}: z_{i} \notin \partial \Omega\right\}
$$

when $s \geq 1 / 2$. The corresponding cardinality $N_{h}$ of $V_{h}$ is equal to the number of nodes in $\mathcal{Z}_{h}$ when $s<1 / 2$ and is otherwise the number of interior nodes in $\Omega$.

Now, let $u_{h}$ denote the solution of the finite element discrete problem given by

Find $u_{h} \in V_{h}$ such that $\mathcal{A}_{s}(u, v)=\langle f, v\rangle$ for all $v \in V_{h}$,

where the bilinear form $\mathcal{A}_{s}(\cdot, \cdot)$ is defined in (3.8) as a special case of the nonlocal bilinear form (2.10) with $\delta=\infty$ and kernel function of the type (2.22) and the right-hand side is defined in the usual manner. The following error estimates are derived in Acosta and Borthagaray (2017). 
If the family of triangulations $\mathcal{T}_{h}$ is globally quasi-uniform, and if $u \in$ $H^{t}(\Omega)$ for $t \in(1 / 2,2]$ and if $0<s \leq t$, then

$$
\left\|u-u_{h}\right\|_{H_{c}^{s}(\Omega)} \leq C h^{t-s}|u|_{H^{t}(\Omega)} .
$$

The estimate (6.1) implies that for $u \in H^{2}(\Omega)$, the expected rate of convergence on a globally quasi-uniform mesh is $h^{2-s}=O\left(N_{h}^{(s-2) / d}\right)$. Because the solutions of (3.5) generally have limited regularity up to the boundary (see Section 3.3.3), it is advisable to use a mesh that is more refined close to the boundary. While using such a mesh can restore the optimal rate of convergence $O\left(N_{h}^{(s-2) / d}\right)$ in $d=1$ dimensions, this does not hold for higherdimensional problems, where shape-regularity of the elements becomes the limiting factor. Therefore we can expect no more than $O\left(N_{h}^{-1 / 2+\varepsilon}\right)$ rate of convergence in $d=2$ dimensions.

Whereas it may be possible to construct an appropriately graded mesh for simple geometries, the general case will require adaptively refined meshes to resolve the boundary singularity. A posteriori error estimators for the integral fractional Laplacian have been developed in Nochetto, von Petersdorff and Zhang (2010), Faustmann, Melenk, Parvizi and Praetorius (2019) and Ainsworth and Glusa (2017).

\subsection{Quadrature rules}

The fractional Poisson equation (3.5) leads to a dense linear algebraic system

$$
\boldsymbol{A}_{s} \vec{u}=\vec{f}
$$

in which the entries in the matrix $\boldsymbol{A}_{s}=\left\{\mathcal{A}_{s}\left(\varphi_{i}, \varphi_{j}\right)\right\}_{i j}$ involve singular integrals. In order to compute these entries, we decompose the integrals into contributions between pairs of elements $K, \widehat{K} \in \mathcal{T}_{h}$ and between pairs consisting of elements $K \in \mathcal{T}_{h}$ and external edges $e \in \partial \mathcal{T}_{h}$ as follows:

$$
\mathcal{A}_{s}\left(\varphi_{i}, \varphi_{i}\right)=\sum_{K} \sum_{\widehat{K}} \mathcal{A}_{s}^{K \times \widehat{K}}\left(\varphi_{i}, \varphi_{j}\right)+\sum_{K} \sum_{e} \mathcal{A}_{s}^{K \times e}\left(\varphi_{i}, \varphi_{j}\right) .
$$

The individual contributions $\mathcal{A}_{s}^{K \times \widehat{K}}$ and $\mathcal{A}_{s}^{K \times e}$ are given by

$$
\begin{aligned}
\mathcal{A}_{s}^{K \times \widehat{K}}\left(\varphi_{i}, \varphi_{j}\right) & =\frac{C(d, s)}{2} \int_{K} \mathrm{~d} \boldsymbol{x} \int_{\widehat{K}} \mathrm{~d} \boldsymbol{x} \frac{\left(\varphi_{i}(\boldsymbol{x})-\varphi_{i}(\boldsymbol{y})\right)\left(\varphi_{j}(\boldsymbol{x})-\varphi_{j}(\boldsymbol{y})\right)}{|\boldsymbol{x}-\boldsymbol{y}|^{d+2 s}}, \\
\mathcal{A}_{s}^{K \times e}\left(\varphi_{i}, \varphi_{j}\right) & =\frac{C(d, s)}{2 s} \int_{K} \mathrm{~d} \boldsymbol{x} \int_{e} \mathrm{~d} \boldsymbol{x} \frac{\varphi_{i}(\boldsymbol{x}) \varphi_{j}(\boldsymbol{x}) \vec{n}_{e} \cdot(\boldsymbol{x}-\boldsymbol{y})}{|\boldsymbol{x}-\boldsymbol{y}|^{d+2 s}}
\end{aligned}
$$

Contributions from non-disjoint pairs of elements (see Figure 6.1) are not directly amenable to numerical quadrature, due to their singular nature. Fortunately, these can be treated by adapting techniques used in the boundary element method (BEM) literature to address similar issues arising from singular kernels (Sauter and Schwab 2010). 


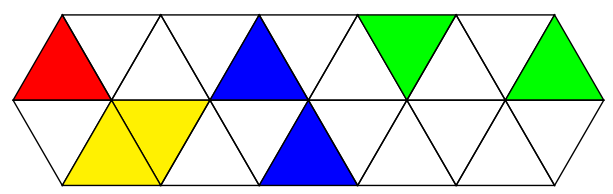

Figure 6.1. Classes of element pairs of configurations that need to be handled: pairs of identical elements (red), element pairs with common edge (yellow), with common vertex (blue) and separated element pairs (green).

However, the fractional Laplacian does pose new difficulties beyond those addressed by the BEM literature, but which can be treated as described by Ainsworth and Glusa (2018b) and Acosta et al. (2017). In particular, Ainsworth and Glusa (2017, 2018b) have developed non-uniform order Gauss-type quadrature rules. Alternatively, one could take the approach proposed by Chernov, von Petersdorff and Schwab (2011). It allows for transforming quadrature rules given on the unit hypercube $[0,1]^{2 d}$ to any pair of elements $K \times \widehat{K}$. Here, the singularity is taken into account through the choice of the weight in the quadrature rules.

Ainsworth and Glusa (2017) gave the following result. Denote the quadrature approximation to the bilinear form $\mathcal{A}_{s}(\cdot, \cdot)$ by $\mathcal{A}_{s}^{Q}(\cdot, \cdot)$. Then, by using $O\left(\log N_{h}^{2 d}\right)$ quadrature points per element pair $K \times \tilde{K}$, the consistency error due to quadrature $\left|\mathcal{A}_{s}(u, v)-\mathcal{A}_{s}^{Q}(u, v)\right|$ is dominated by the discretization error.

A brief discussion about solvers and condition numbers for finite element discretizations of the integral fractional Laplacian is given in Section 12.2.

\section{Finite element methods for the spectral fractional Laplacian}

The extension problem (3.16) has been extensively used to solve equations involving the spectral fractional Laplacian. For a bounded domain, the extension problem is posed on $\Omega_{z}=\Omega \times[0, \infty)$ and takes the form

$$
\begin{cases}-\nabla \cdot z^{\beta} \nabla U(\boldsymbol{x}, z)=0 & \text { for all }(\boldsymbol{x}, z) \in \Omega_{z}, \\ U(\boldsymbol{x}, z)=0 & \text { for all }(\boldsymbol{x}, z) \in \partial_{L} \Omega_{z}, \\ \frac{\partial U}{\partial n^{\beta}}(\boldsymbol{x})=d_{s} f(\boldsymbol{x}) & \text { for all } \boldsymbol{x} \in \Omega,\end{cases}
$$

where $d_{s}$ is the constant given in (3.16) and where $\partial_{L} \Omega_{z}:=\partial \Omega \times[0, \infty)$ denotes the lateral surface of the semi-infinite cylinder. The solution $u$ to the spectral fractional Poisson problem (3.14) is then recovered by taking the trace on $\Omega$, i.e. $u=\operatorname{trace}_{\Omega} U$. 
We define the solution space $\mathcal{H}_{\beta}^{1}\left(\Omega_{z}\right)$ on the semi-infinite cylinder $\Omega_{z}$ as

$$
\mathcal{H}_{\beta}^{1}\left(\Omega_{z}\right)=\left\{V \in H_{z^{\beta}}^{1}\left(\Omega_{z}\right): V=0 \text { on } \partial_{L} \Omega_{z}\right\},
$$

with norm $\|V\|_{\mathcal{H}_{\beta}^{1}}=|V|_{H_{z \beta}^{1}}$. The weak form of the extension problem is then as follows:

$$
\text { Find } U \in \mathcal{H}_{\beta}^{1}\left(\Omega_{z}\right) \text { such that } \int_{\Omega_{z}} z^{\beta} \nabla U \cdot \nabla V=d_{s}\left\langle f, \operatorname{tr}_{\Omega} V\right\rangle
$$

for all $V \in \mathcal{H}_{\beta}^{1}\left(\Omega_{z}\right)$.

In the literature, the fact that the domain is unbounded in the $z$-direction has been handled in different ways. One class of methods exploits the fact that truncation of the domain to $\Omega_{z_{\text {trun }}}:=\Omega \times\left[0, z_{\text {trun }}\right]$ can be shown to be exponentially converging in $z_{\text {trun }}$, and then discretizes the domain $\Omega_{z_{\text {trun }}}$ using finite elements. A second class uses a hybrid approach and discretizes using finite elements in the $\boldsymbol{x}$-direction and a suitable spectral method in the $z$-direction. We will describe both these methods below.

\subsection{Truncation in the extended direction}

The truncation approach was pioneered by Nochetto, Otárola and Salgado (2015). A first result shows that truncation of the semi-infinite cylinder $\Omega_{z}$ only leads to an exponentially small error.

Let $U \in \mathcal{H}_{\beta}^{1}$ denote the solution of $(7.2)$ and let $U_{z_{\text {trun }}}$ be the extension by zero to $\Omega_{z}$ of the solution to (7.2) posed on the truncated domain $\Omega_{z_{\text {trun }}}$, $z_{\text {trun }} \geq 1$, with a homogeneous Dirichlet condition enforced at $z=z_{\text {trun }}$. Then

$$
\left\|U-U_{z_{\text {trun }}}\right\|_{\mathcal{H}_{\beta}^{1}\left(\Omega_{z}\right)} \leq C \mathrm{e}^{-\sqrt{\lambda_{0}} z_{\text {trun }} / 4}\|f\|_{H^{-s}(\Omega)},
$$

where $\lambda_{0}$ is the smallest eigenvalue of the integer-order Laplacian as given by (3.13).

Now, let $\mathcal{T}_{h}$ denote a quasi-uniform mesh of $\Omega$ with mesh size $h$ and define a graded mesh $\mathcal{T}_{z_{\text {trun }}}$ of the interval $\left[0, z_{\text {trun }}\right]$ by

$$
z_{k}=(k / M)^{\gamma} z_{\text {trun }}, \quad k=0, \ldots, M,
$$

with $M \sim h^{-1}$ and $\gamma>3 /(1-\beta)$. Then define a mesh $\mathcal{T}$ on the extended domain $\Omega_{z_{\text {trun }}}$ as the tensor product of $\mathcal{T}_{h}$ and $\mathcal{T}_{z_{\text {trun }}}$, and let $V_{h}$ denote the space of piecewise linear finite element functions on $\mathcal{T}$. Let $N=\operatorname{dim} V_{h}$ be the overall number of degrees of freedom and choose the truncation to be $z_{\text {trun }} \sim|\log N|$. Then the following convergence result holds.

Let $f \in H_{c}^{1-s}(\Omega), U \in \mathcal{H}_{\beta}^{1}\left(\Omega_{z}\right)$ be the solution of (7.2), and let $U_{h} \in V_{h}$ be the solution of the restriction of (7.2) to $V_{h}$. Moreover, let $u=\operatorname{trace}_{\Omega} U$ 
denote the solution to the spectral fractional-order Poisson problem (3.14), and let $u_{h}=\operatorname{trace}_{\Omega} U_{h}$ be its discrete approximation. Then

$$
\begin{aligned}
\left\|u-u_{h}\right\|_{H_{c}^{s}(\Omega)} & \leq C\left\|U-U_{h}\right\|_{\mathcal{H}_{\beta}^{1}\left(\Omega_{z}\right)} \\
& \leq C|\log h|^{s} h\|f\|_{H_{c}^{1-s}(\Omega)} \\
& \leq C|\log N|^{s} N^{-1 /(d+1)}\|f\|_{H_{c}^{1-s}(\Omega)} .
\end{aligned}
$$

As observed by Nochetto et al. (2015), this shows that the result is optimal in terms of regularity but sub-optimal in terms of complexity. This shortcoming can be addressed using sparse grids (Banjai et al. 2019) or $h p$-finite elements (Meidner, Pfefferer, Schürholz and Vexler 2018, Banjai et al. 2019). Using the latter, exponential convergence can be obtained, including for cases with low regularity of the domain and incompatibility of the forcing term (Banjai et al. 2019).

\subsection{Spectral method in the extended direction}

A different approach was taken by Ainsworth and Glusa (2018a). Instead of truncating the domain, a spectral method is used for the extended direction, based on the observation that the eigenfunctions of the extension problem (7.1) are given by

$$
\varphi_{m}(\boldsymbol{x}) \psi_{m}(z), \quad m \in \mathbb{N},
$$

where

$$
\psi_{m}(z)=c_{s}\left(\lambda_{m}^{1 / 2} z\right)^{s} K_{s}\left(\lambda_{m}^{1 / 2} z\right),
$$

and where $c_{s}=2^{1-s} / \Gamma(s),\left(\varphi_{m}, \lambda_{m}\right)$ are the eigenpairs of (3.13) and $K_{\nu}$ are the modified Bessel functions of the second kind.

This motivates the choices

$$
\begin{aligned}
V_{h} & =\left\{v_{h} \in H_{0}^{1}(\Omega):\left.v_{h}\right|_{K} \in \mathbb{P}_{k}(K) \text { for all } K \in \mathcal{T}_{h}\right\}, \\
V_{h, M} & =\left\{V_{h, M}=\sum_{m=0}^{M-1} v_{h, m}(\boldsymbol{x}) \widehat{\psi}_{m}(z): v_{h, m} \in V_{h}\right\} \subset \mathcal{H}_{\beta}^{1}\left(\Omega_{z}\right),
\end{aligned}
$$

where

$$
\widehat{\psi}_{m}(z):=c_{s}\left(\widehat{\lambda}_{m}^{1 / 2} z\right)^{s} K_{s}\left(\widehat{\lambda}_{m}^{1 / 2} z\right)
$$

are defined using suitable approximations $\widehat{\lambda}_{m}$ instead of the true eigenvalues $\lambda_{m}$.

It turns out that using the asymptotic law for the eigenvalues of the Laplacian and using a coarse finite element approximation gives sufficiently 
good approximations $\widehat{\lambda}_{m}$. Moreover, by decimation, only $|\log h|^{p}$ for some $p>0$ eigenvalue approximations is required. Overall, this results in the following error estimate.

Let $f \in H_{c}^{r}(\Omega), r \geq-s$, let $U \in \mathcal{H}_{\beta}^{1}\left(\Omega_{z}\right)$ be the solution of $(7.2)$ and let $U_{h} \in V_{h}$ be the solution of the restriction of (7.2) to $V_{h}$. Moreover, let $u=\operatorname{trace}_{\Omega} U$ be the solution to the spectral fractional-order Poisson problem (3.14) and let $u_{h}=\operatorname{trace}_{\Omega} U_{h}$ be its discrete approximation. Then

$$
\begin{aligned}
\left\|u-u_{h}\right\|_{H_{c}^{s}(\Omega)} & \leq C\left\|U-U_{h, M}\right\|_{\mathcal{H}_{\beta}^{1}\left(\Omega_{z}\right)} \\
& \leq C|f|_{H_{c}^{r}(\Omega)} h^{\min \{k, r+s\}} \sqrt{|\log h|} \\
& =C|f|_{H_{c}^{r}(\Omega)} N^{-\min \{k, r+s\} / d}|\log N|^{p} .
\end{aligned}
$$

Remark 7.1 (solution of the linear system). Provided that the discretization of the extension problem (7.1) displays a tensor structure, the solution of the arising linear system reduces to a sequence of discretized integer-order reaction-diffusion-type problems. These problems are readily solved using classical iterative linear solvers such as conjugate gradient and multigrid; see e.g. Chen, Nochetto, Otárola and Salgado (2016a) and Ainsworth and Glusa (2018a).

\section{Spectral-Galerkin methods for nonlocal diffusion}

Compared with its fractional counterparts discussed in Section 9, algorithms and numerical analyses of spectral method for nonlocal diffusion have been much less studied. Here, we simply consider a Fourier spectral-Galerkin method developed by Du and Yang (2016, 2017).

For simplicity, we focus on the one-dimensional periodic nonlocal diffusion problem given by

$$
-\left(\mathcal{L}_{\delta} u\right)(x)=f(x) \text { for all } x \in(-\pi, \pi)
$$

with periodic boundary condition, where we express the nonlocal operator $\mathcal{L}_{\delta}$ in the equivalent form

$$
\left(\mathcal{L}_{\delta} u\right)(x)=2 \int_{|z| \leq \delta} \gamma_{\delta}(z)(u(x+z)-u(x)) \mathrm{d} z
$$

where $f(x) \in L_{\text {per }}^{2}[-\pi, \pi]$, where $L_{\text {per }}^{2}[-\pi, \pi]$ denotes the space of periodic functions in $L^{2}[-\pi, \pi]$ having zero mean. As in the earlier sections, $\delta$ denotes the horizon. Here, the kernel function $\gamma_{\delta}(z)=\gamma_{\delta}(|z|)$ is defined by (5.2) and (5.3) with $d=1$, which ensures that as the horizon $\delta$ tends to zero, the nonlocal operator $\mathcal{L}_{\delta}$ reduces to the PDE Laplacian, i.e. $\mathrm{d}^{2} / \mathrm{d} x^{2}$ in one dimension. 
For any positive integer $n, \mathrm{e}^{ \pm \mathrm{i} n x}$ are eigenfunctions of $-\mathcal{L}_{\delta}$ under periodic boundary conditions with the corresponding eigenvalues ${ }^{2}$

$$
\lambda_{n, \delta}=4 \int_{0}^{\delta} \gamma_{\delta}(z)(1-\cos (n z)) \mathrm{d} z
$$

Let $X_{N}^{0}:=\operatorname{span}\left\{\mathrm{e}^{ \pm \mathrm{i} n x}\right\}_{n=1,2, \ldots, N}$ and let

$$
v_{N}(x)=\sum_{n=1}^{N} \widehat{v}_{n} \mathrm{e}^{\mathrm{i} n x}+\widehat{v}_{-n} \mathrm{e}^{-\mathrm{i} n x} \in X_{N}^{0}
$$

for any set of constants $\left\{\widehat{v}_{n}\right\}$. Note that because, for any nonzero integer $n$, $\mathrm{e}^{\mathrm{i} n x}$ has zero mean with respect to any interval of length $2 \pi$, so does $v_{N}(x)$. We have that

$$
-\mathcal{L}_{\delta} v_{N}=\sum_{n=1}^{N} \lambda_{n, \delta}\left(\widehat{v}_{n} \mathrm{e}^{\mathrm{i} n x}+\widehat{v}_{-n} \mathrm{e}^{-\mathrm{i} n x}\right) \quad \text { for } \delta \geq 0
$$

The Fourier spectral-Galerkin scheme of Du and Yang $(2016,2017)$ is as follows:

Given $f(x) \in L_{\text {per }}^{2}[-\pi, \pi]$, seek $u_{N, \delta}(x) \in X_{N}^{0}$ such that

$$
\left(-\mathcal{L}_{\delta} u_{N, \delta}, v_{N}\right)=\left(f, v_{N}\right) \text { for all } v_{N} \in X_{N}^{0}
$$

or equivalently

$$
u_{N, \delta}=-\mathcal{L}_{\delta}{ }^{-1} P_{N} f=\sum_{n=1}^{N} \lambda_{n, \delta}^{-1}\left(\widehat{f}_{n} \mathrm{e}^{\mathrm{i} n x}+\widehat{f}_{-n} \mathrm{e}^{-\mathrm{i} n x}\right),
$$

where $(\cdot, \cdot)$ denotes the standard $L^{2}(-\pi, \pi)$ inner product and $P_{N}$ denotes the standard spectral Fourier projection onto $X_{N}:=\operatorname{span}\left\{\mathrm{e}^{ \pm \mathrm{i} n x}\right\}_{n=0,1, \ldots, N}$ of an element in $L^{2}[-\pi, \pi]$.

Du and Yang (2016, Lemma 1) proved that the numerical scheme (8.4) is asymptotically compatible. In particular, they proved that

$$
\left\|u_{N, \delta}-u_{N, 0}\right\|_{L^{2}(-\pi, \pi)} \leq C \delta^{2}\left\|P_{N} f\right\|_{L^{2}(-\pi, \pi)},
$$

where the constant $C$ is independent of $N$ and $\delta$. Here, $u_{N, 0}$ denotes the Fourier spectral solution in $X_{N}^{0}$ of the local diffusion problem

$$
-\mathcal{L}_{0} u(x):=-u_{x x}(x)=f(x) \text { for all } x \in(-\pi, \pi)
$$

${ }^{2}$ Note that $-\mathrm{d}^{2} / \mathrm{d} x^{2}$ has the same eigenfunctions $\mathrm{e}^{ \pm \mathrm{in} x x}$, as does $-\mathcal{L}_{\delta}$, and has the eigenvalues $n^{2}$. Note that, in the limit $\delta \rightarrow 0, \lambda_{n, \delta} \rightarrow \lambda_{n, 0}:=n^{2}$. 
with the periodic boundary condition. The proof of the estimate (8.5) follows from the error representation

$$
\left\|u_{N, \delta}-u_{N, 0}\right\|_{L^{2}(-\pi, \pi)}^{2}=\sum_{n=1}^{N}\left|\frac{1}{\lambda_{n, \delta}}-\frac{1}{\lambda_{n, 0}}\right|^{2}\left(\left|\widehat{f}_{n}\right|^{2}+\left|\widehat{f}_{-n}\right|^{2}\right)
$$

and a careful analysis of the asymptotic behaviour of $\lambda_{n, \delta}$.

Let $u_{0}(\boldsymbol{x})$ denote the exact solution of the local problem (8.6). Then, using standard error estimation theories for local models, it is easy to show that $u_{N, 0}$ converges in the $L^{2}$ sense to $u_{0}$ at least quadratically with respect to $1 / N$, provided that $f \in L^{2}(-\pi, \pi)$. As a result, we arrive at

$$
\left\|u_{N, \delta}-u_{0}\right\|_{L^{2}(-\pi, \pi)} \leq C\left(\delta^{2}+N^{-2}\right)\|f\|_{L^{2}(-\pi, \pi)},
$$

with a generic constant $C$ independent of $\delta, N, f, u_{0}$ and $u_{\delta}$, where $u_{\delta}$ denotes the exact solution of (8.1). This estimate indicates that the Fourier spectral method of Du and Yang $(2016,2017)$ is asymptotically compatible. Moreover, we obtain a uniform error estimate in the sense that the estimates hold for any sufficiently small $\delta$ and any sufficiently large $N$ without any restriction on the relative sizes of $\delta$ and $N$.

These one-dimensional results can be easily extended to higher-dimensional nonlocal diffusion problems in rectangular and rectangular parallelepiped domains with periodic boundary conditions. See Du and Yang (2017) for discussions about two and three-dimensional problem. Furthermore, the analysis was extended to the nonlocal Allen-Cahn equations (Du and Yang 2016, Section 4).

The eigenvalues $\lambda_{n, \delta}$ defined by (8.3) cannot, in general, be determined analytically. Thus, when implementing the scheme given in (8.4), those eigenvalues $\lambda_{n, \delta}$ have to be estimated. The numerical evaluation of the integral in (8.3) is challenging because $\cos (n z)$ is highly oscillatory for large $n$. As an example, we focus on a kernel that is singular at the origin, namely,

$$
\gamma_{\delta}(z)=\frac{C_{\beta}}{\delta^{3-\beta}|z|^{\beta}} \quad \text { for all } z \in[-\delta, 0) \cup(0, \delta] \text { and for } \beta \in(0,3) .
$$

Then, by Taylor expansion, we have that

$$
\begin{aligned}
\lambda_{n, \delta} & =-\frac{4 C_{\beta}}{\delta^{2}} \int_{0}^{1} r^{-\beta}(\cos (n \delta r)-1) \mathrm{d} r \\
& =-\frac{4 C_{\beta}}{\delta^{2}} \sum_{k=1}^{\infty} \frac{(-1)^{k}|n \delta|^{2 k}}{(2 k) !(2 k+1-\beta)} \\
& :=-\frac{4 C_{\beta}}{\delta^{2}} K(n \delta) .
\end{aligned}
$$

Therefore it suffices to compute $K(n \delta)$ accurately. Du and Yang (2017) have given a hybrid algorithm for computing $K(n \delta)$. If $n \delta$ is 'small' then 
the series in (8.9) converges fast, so one may approximate $K(n \delta)$ by using a truncation of that series. On the other hand, for $n \delta$ 'large', Du and Yang (2017) observed that $K(n \delta)$ is a solution of the ordinary differential equation

$$
K^{\prime}(z)=\frac{z-1}{z} K(z)+\frac{\cos (z)-1}{z} \text { on }(0, n \delta]
$$

evaluated at $z=n \delta$. For a starting condition, $K(1)$ may be used; it can be computed using a truncation of the series in (8.9). As a result, $K(n \delta)$ can be accurately evaluated for large $n \delta$ by using, for example, a high-order Runge-Kutta method.

Slevinsky et al. (2018) proposed a Fourier spectral method to solve nonlocal diffusion models on the unit sphere $\mathcal{S}^{2} \subset \mathbb{R}^{3}$, where the nonlocal Laplace-Beltrami operator is defined by

$$
\mathcal{L}_{\delta} u(x)=2 \int_{\mathcal{S}^{2}}(u(x+z)-u(x)) \gamma_{\delta}(z) \mathrm{d} \nu(z),
$$

where $\nu$ denotes the standard measure on $\mathcal{S}^{2}$. The basic idea is to apply spherical harmonics (Atkinson and Han 2012):

$$
Y_{l}^{m}(x)=Y_{l}^{m}(\theta, \varphi)=\frac{\mathrm{e}^{\mathrm{i} m \varphi}}{\sqrt{2 \pi}} \mathrm{i}^{m+|m|} \sqrt{\left(l+\frac{1}{2}\right) \frac{(l-m) !}{(l+m) !}} P_{l}^{m}(\cos \theta)
$$

with $l \geq 0$ and $-l \leq m \leq l$, for which it is shown that they are the eigenfunctions of the nonlocal Laplace-Beltrami operator. Here $(\theta, \varphi)$ are the spherical coordinates of $x \in \mathbb{S}^{2}$ and $P_{l}$ denote the associated Legendre polynomials. Specifically, there exists $\mathcal{L}_{\delta} Y_{l}^{m}(x)=\lambda_{\delta}(l) Y_{l}^{m}(x)$ with

$$
\lambda_{\delta}(l)=4 \pi \int_{-1}^{1}\left(P_{l}(y)-1\right) \gamma_{\delta}(\sqrt{2(1-y)}) \mathrm{d} y .
$$

To compute the eigenvalue $\lambda_{\delta}(l)$, one evaluates an integral which is highly oscillatory due to the Legendre polynomials. Slevinsky et al. (2018) proposed a numerical scheme by using a modified Clenshaw-Curtis quadrature rule with the computation complexity $O\left(l^{2}\right)$ per eigenvalue $\lambda_{\delta}(l)$. For sufficiently large $l$, they applied Szegö's asymptotic formula for Legendre polynomials, which reduces the complexity to $O(l \log l)$ per eigenvalue. Then a fast spherical harmonic transform (Slevinsky 2019) was applied to accelerate synthesis and analysis of the series expansion in spherical harmonics.

Remark 8.1 (extension to phase field methods). The study of spectral approximation to nonlocal problems has been extended to nonlinear models such as phase field equations (Du and Yang 2016, Du, Ju, Li and Qiao 2018b). Conventional phase field (diffuse interface) models take on a 
free energy of the type (Du and Feng 2020)

$$
E^{0}(u)=\int_{\Omega}\left(\frac{\epsilon}{2}|\nabla u(x)|^{2}+\frac{1}{4 \epsilon}(u(x)-1)^{2}\right) \mathrm{d} x .
$$

The nonlocal version of the free energy can be written as (Du and Yang 2016)

$$
E^{\delta}(u)=\int_{\Omega} \int_{\Omega}\left(\frac{\epsilon}{2} \gamma_{\delta}(|s|) \frac{(u(x+s)-u(x))^{2}}{2} \mathrm{~d} s+\frac{1}{4 \epsilon}(u(x)-1)^{2}\right) \mathrm{d} x,
$$

which recovers $E^{0}$ as its gamma limit for $\delta \rightarrow 0$.

A nonlocal Allen-Cahn equation can be defined as the $L^{2}$ gradient flow of the energy in (8.13). The convergence of AC spectral approximations for problems defined on periodic domains has been established in Du and Yang (2016). With the assumption on smooth minimizers, spectral accuracy can be assured. When nonlocal interaction kernels do not have sufficiently strong singularities, the minimizers of the nonlocal free energy (subject to a total mass condition) may develop a discontinuous profile, in contrast to the conventional diffuse interface models with smooth phase field functions. However, the convergence of spectral approximations can be assured even for such cases. We note that, historically, (8.12) is often derived from a nonlocal form (8.13) via the Landau expansion; see the additional discussions on this perspective given in $\mathrm{Du}$ (2019). Naturally, one may also consider the $H^{-1}$ gradient flow of the nonlocal energy or gradient flows with respect to a nonlocal space embedded between $H^{-1}$ and $L^{2}$; some related numerical analysis can be found in Li, Qiao and Wang (2019) and Ainsworth and Mao (2017).

Remark 8.2. Nonlocal diffusion allows for singular solutions, which pose new challenges in the design of numerical discretizations. Whereas spectral methods are shown to possess many good properties such as asymptotic compatibility and are able to capture the discontinuities in solutions, spurious Gibbs phenomena do appear. Given the development of spectral methods in the context of PDEs that are effective in singularity detection and in high-order recovery of solution information near and away from singularities (see e.g. Tadmor 2007), it is natural to pursue further studies of how to adopt and extend those techniques to nonlocal models.

Furthermore, the algorithms introduced in this section focus on periodic boundary conditions and allows for the derivation of uniform convergence rates with respect to both $\delta$ and $N$; see e.g. (8.7). The extension to other types of boundary conditions, e.g. Dirichlet or Neumann volume constraints, remains an open problem. The main difficulty stems from the lack of a closed expression for the eigenvalues analogous to the expression given in (8.3). Hence the difference $\lambda_{n, \delta}^{-1}-\lambda_{0, \delta}^{-1}$ is difficult to estimate. An investigation of the AC property of spectral method in such settings is also warranted. 


\section{Spectral-Galerkin methods for fractional diffusion}

In this section we consider spectral methods for determining approximate solutions of fractional diffusion problems. We first address the fractional Poisson problem posed on $\Omega=\mathbb{R}^{d}$. In this case, the solution decays slowly with algebraic rates as $|\boldsymbol{x}| \rightarrow \infty$, even if the source term is compactly supported. As a result, some traditional discretization strategies used for the local PDE diffusion problem are not applicable here. Subsequently, we consider fractional diffusion in a bounded domain $\Omega$ for which the solution possesses a weakly singular layer near the boundary $\partial \Omega$, even if the source term is smooth. This property engenders many challenges in the development and analysis of numerical schemes. Compared to, say, finite difference and finite element methods, spectral methods with specially constructed basis functions may approximate the solution of fractional diffusion problems on bounded domains with higher accuracy.

\subsection{Spectral-Galerkin methods in unbounded domains}

For $s \in(0,1)$, we consider the fractional diffusion problem in $\Omega=\mathbb{R}^{d}$ given by

$$
\begin{cases}(-\Delta)^{s} u(\boldsymbol{x})+c u(\boldsymbol{x})=f(\boldsymbol{x}) & \text { for all } \boldsymbol{x} \in \mathbb{R}^{d}, \\ u(\boldsymbol{x}) \rightarrow 0 & \text { as }|\boldsymbol{x}| \rightarrow \infty,\end{cases}
$$

where $c$ denotes a given positive constant and $f$ denotes a given source term. A weak formulation of problem (9.1) is as follows:

Given a positive constant $c$ and $f \in H^{-s}\left(\mathbb{R}^{d}\right)$, seek $u \in H^{s}\left(\mathbb{R}^{d}\right)$ such that

$$
\mathcal{A}_{s}(u, v)=(f, v) \quad \text { for all } v \in H^{s}\left(\mathbb{R}^{d}\right),
$$

where, for all $u, v \in H^{s}\left(\mathbb{R}^{d}\right)$,

$$
\mathcal{A}_{s}(u, v)=\frac{C_{d, s}}{2} \int_{\mathbb{R}^{d}} \int_{\mathbb{R}^{d}} \frac{(u(\boldsymbol{y})-u(\boldsymbol{x}))(v(\boldsymbol{y})-v(\boldsymbol{x}))}{|\boldsymbol{y}-\boldsymbol{x}|^{d+2 s}} \mathrm{~d} \boldsymbol{y} \mathrm{d} \boldsymbol{x}+c(u, v)
$$

with $C_{d, s}$ is defined in (3.3). The well-posedness of problem (9.2) follows directly from the Lax-Milgram theorem.

Several successful strategies have been developed for determining approximations of solutions of local PDE diffusion problems posed on $\mathbb{R}^{d}$; see e.g. the survey paper by Shen and Wang (2009) and the references cited therein. One way is to truncate the domain $\mathbb{R}^{d}$, provided that the solution decays rapidly. However, it is known that solution of the fractional diffusion equation decays slowly with a power law at infinity so that the naive truncation 
approach results in a relatively large error due to domain truncation. ${ }^{3}$ Another approach is to design an artificial boundary condition imposed on the boundary of a truncated domain that results in the same solution within a bounded region; the use of this approach remains largely an open question in the fractional case. A third approach, which is the approach used here, does not involve domain truncation, but instead approximates the solution in terms of orthogonal functions that potentially can well approximate the solution in the unbounded domain.

For simplicity, we restrict our discussion to the one-dimensional case, i.e. $d=1$; higher-dimensional cases can be treated in a similar manner by using tensor products of one-dimensional orthogonal polynomials.

\subsubsection{Approximation by Hermite polynomials}

The orthonormal Hermite polynomials $\left\{H_{n}(x)\right\}$ are defined by the threeterm recurrence relation (Abramowitz and Stegun 1972, Mao and Shen 2017)

$$
\begin{aligned}
H_{0} & =\pi^{-1 / 4}, \quad H_{1}(x)=\sqrt{2} \pi^{-1 / 4} x, \\
H_{n+1} & =x \sqrt{\frac{2}{n+1}} H_{n}(x)-\sqrt{\frac{n}{n+1}} H_{n-1}(x) \quad \text { for } n=1,2, \ldots .
\end{aligned}
$$

It is well known that those polynomials form an orthonormal basis in the weighted space $L_{w}^{2}(\mathbb{R})$, where the weight function is given by $w(x)=\mathrm{e}^{-x^{2}}$. Let $V_{N}^{H}$ denote the space spanned by the Hermite polynomials of degree less than or equal to $N$, and let

$$
X_{N}^{H}=\left\{v: \sqrt{w(x)} u(x) \text { for all } u \in V_{N}^{H}\right\} .
$$

We define the $L_{w}^{2}(\mathbb{R})$-projection operator $Q_{N}^{H}: L_{w}^{2}(\mathbb{R}) \rightarrow X_{N}^{H}$ by

$$
\int_{\mathbb{R}} u(x) v_{N}(x) w(x) \mathrm{d} x=\int_{\mathbb{R}}\left(Q_{N}^{H} u\right)(x) v_{N}(x) w(x) \mathrm{d} x \quad \text { for all } v_{N} \in X_{N}^{H} .
$$

We also define the modified operator $\widehat{Q}_{N}^{H}: L^{2}(\mathbb{R}) \rightarrow X_{N}^{H}$ by

$$
\widehat{Q}_{N}^{H} u=w^{1 / 2} Q_{N}^{H}\left(u w^{-1 / 2}\right) \text { for all } u \in L^{2}(\mathbb{R}) .
$$

Then we have the approximation property

$$
\left\|\widehat{Q}_{N}^{H} u-u\right\|_{H^{s}(\mathbb{R})} \leq C N^{(\ell-m) / 2}\|u\|_{\widehat{H}^{m}(\mathbb{R})} \quad \text { for all } s \in[0, m],
$$

${ }^{3}$ However, see Remark 3.1, where it is pointed out that truncated domains may be of interest in their own right. 
where $C>0$ does not depend on $u$ or $N$ and where the modified Sobolev space $\widehat{H}^{m}(\mathbb{R})$ comes equipped with the norm and seminorm

$$
\begin{array}{r}
\|u\|_{\widehat{H}^{m}(\mathbb{R})}^{2}=\sum_{0 \leq k \leq m}\left\|\left(\frac{\mathrm{d}}{\mathrm{d} x}+x\right)^{k} u\right\|_{L^{2}(\mathbb{R})}^{2}, \\
|u|_{\widehat{H}^{m}(\mathbb{R})}=\left\|\left(\frac{\mathrm{d}}{\mathrm{d} x}+x\right)^{m} u\right\|_{L^{2}(\mathbb{R})}^{2},
\end{array}
$$

respectively.

The Hermite-Galerkin discretization of the fractional diffusion problem (9.1) is then as follows:

Given a positive constant $c$ and $f \in H^{-s}\left(\mathbb{R}^{d}\right)$, seek $u_{N}(x) \in X_{N}^{H}$ such that

$$
\mathcal{A}_{s}\left(u_{N}, v_{N}\right)=\left(I_{N}^{H} f, v_{N}\right) \text { for all } v_{N} \in X_{N}^{H},
$$

where $I_{N}^{H}: C(\mathbb{R}) \rightarrow X_{N}^{H}$ denotes the Gauss-Hermite interpolation operator with respect to the Gauss-Hermite points (Shen, Tang and Wang 2011). Then the standard energy estimate and the approximation property (9.3) result in the error estimate

$$
\left\|u-u_{N}\right\|_{H^{s}(\mathbb{R})} \leq C N^{s-m}|u|_{\widehat{H}^{m}(\mathbb{R})}+C N^{1 / 6-\ell / 2}|f|_{\widehat{H}^{\ell}(\mathbb{R})},
$$

provided that $u \in \widehat{H}^{m}(\mathbb{R})$ and $f \in \widehat{H}^{\ell}(\mathbb{R})$, where again $C>0$ does not depend on $u, f$ or $N$. This estimate indicates that the numerical solution fails to converge exponentially, because either $u$ and or $f$ will decay algebraically as $x \rightarrow \infty$; see Mao and Shen (2017, Section 5).

The Hermite-Galerkin discretization (9.4) is simple to implement because the mass matrix is diagonal, whereas the stiffness matrix can be computed efficiently using the Fourier transform. An implementation of the HermiteGalerkin method (9.4) using Fourier transforms is provided in Mao and Shen (2017). See also Mao and Shen (2017) and Tang, Yuan and Zhou (2018) for related Hermite collocation methods, whose error estimate also depend on the Fourier transform of the solution $u$ and the source term $f$.

\subsubsection{Approximation by modified Gegenbauer polynomials}

In Tang, Wang, Yuan and Zhou (2019), the approach taken in Section 9.1.1 is extended to approximations in which the basis is constructed by using Gegenbauer polynomials that are modified using a nonlinear singular mapping. The resulting basis has proved to be better suited to approximating functions with algebraic decay rates (see e.g. Boyd 1987, Boyd 2001) when compared to classical bases of orthogonal polynomials such as Hermite or Laguerre polynomials. 
The Gegenbauer polynomials, denoted by $G_{n}^{\lambda}(t)$ with $t \in(-1,1)$ and scaling parameter $\lambda>-1 / 2$, are defined by the three-term recurrence relation

$$
\begin{aligned}
G_{0}^{\lambda}(t) & =1, \quad G_{n}^{\lambda}(t)=2 \lambda t, \\
n G_{n}^{\lambda}(t) & =2 t(n+\lambda-1) G_{n-1}^{\lambda}(t)-(n+2 \lambda-2) G_{n-2}^{\lambda}(t), \quad n=2,3, \ldots .
\end{aligned}
$$

The Gegenbauer polynomials are orthogonal with respect to the weight function $w_{\lambda}(t)=\left(1-t^{2}\right)^{\lambda-1 / 2}$, and they are closely related to the hypergeometric functions (Gradshteyn and Ryzhik 2007, page 1000) that are widely used for the analysis of fractional differential equations; see e.g. Ervin, Heuer and Roop (2018).

We seek approximate solutions in the space

$$
X_{N}^{G}=\operatorname{span}\left\{R_{n}^{\lambda}(x): n=0,1, \ldots, N\right\},
$$

where the mapped Gegenbauer functions $R_{n}^{\lambda}(x)$ are defined by

$$
R_{n}^{\lambda}(x):=\left(1+x^{2}\right)^{-\frac{\lambda+1}{2}} G_{n}^{\lambda}\left(\frac{x}{\sqrt{1+x^{2}}}\right) \text { for all } x \in \mathbb{R} .
$$

The $L^{2}(\mathbb{R})$-orthogonal projection operator $Q_{N}^{G}: L^{2}(\mathbb{R}) \rightarrow X_{N}^{G}$ is defined by

$$
\int_{\mathbb{R}} u(x) v_{N}(x) \mathrm{d} x=\int_{\mathbb{R}} Q_{N}^{G} u(x) v_{N}(x) \mathrm{d} x \quad \text { for all } v_{N} \in X_{N}^{G} .
$$

Next, let $B_{m}^{G}(\mathbb{R})$ denote the weighted Sobolev space defined by the norm and seminorm

$$
\begin{aligned}
\|u\|_{B_{m}^{G}(\mathbb{R})}^{2} & =\sum_{0 \leq k \leq m}\left\|\left(1+x^{2}\right)^{\frac{1}{4}-\frac{\lambda+m}{2}}\left(\left(1+x^{2}\right)^{\frac{3}{2}} \frac{\mathrm{d}}{\mathrm{d} x}\right)^{k}\left(\left(1+x^{2}\right)^{\frac{\lambda+1}{2}} u\right)\right\|_{L^{2}(\mathbb{R})}^{2}, \\
|u|_{B_{m}^{G}(\mathbb{R})} & =\left\|\left(1+x^{2}\right)^{\frac{1}{4}-\frac{\lambda+m}{2}}\left(\left(1+x^{2}\right)^{\frac{3}{2}} \frac{\mathrm{d}}{\mathrm{d} x}\right)^{m}\left(\left(1+x^{2}\right)^{\frac{\lambda+1}{2}} u\right)\right\|_{L^{2}(\mathbb{R})}^{2},
\end{aligned}
$$

respectively. Then, for any $u \in H^{s}(\mathbb{R}) \cap B_{m}^{G}(\mathbb{R})$ with integer $1 \leq m \leq N+1$, $s \in(0,1)$ and $\lambda>-1 / 2$, we have the approximation property

$$
\left\|Q_{N}^{G} u-u\right\|_{H^{s}(\mathbb{R})} \leq C N^{s-m}|u|_{B_{m}^{G}(\mathbb{R})},
$$

where $C>0$ does not depend on $N$ and $u$.

The mapped Gegenbauer-Galerkin discretization of the fractional diffusion problem (9.1) is as follows:

Given a positive constant $c$ and $f \in H^{-s}\left(\mathbb{R}^{d}\right)$, seek $u_{N}(x) \in X_{N}^{G}$ such that

$$
\mathcal{A}_{s}\left(u_{N}, v_{N}\right)=\left(I_{N}^{G} f, v_{N}\right) \text { for all } v_{N} \in X_{N}^{G},
$$


where $I_{N}^{G}: C(\mathbb{R}) \rightarrow X_{N}^{G}$ denotes the mapped Gegenbauer-Gauss interpolation operator based on the Gegenbauer-Gauss quadrature points (Shen et al. 2011, Chapter 3). Then, for any $u \in H^{s}(\mathbb{R}) \cap B_{m}^{G}(\mathbb{R})$ and $f \in B_{\ell}^{G}(\mathbb{R})$ with integers $1 \leq m, \ell \leq N+1$ and $\lambda>-1 / 2$, we have the error estimate

$$
\left\|u-u_{N}\right\|_{H^{s}(\mathbb{R})} \leq C N^{s-m}|u|_{B_{m}^{G}(\mathbb{R})}+C N^{-\ell}|f|_{B_{\ell}^{G}(\mathbb{R})},
$$

where $C$ denotes a positive constant having value independent of $N, u$ and $f$. The proof relies on the standard energy estimate as well as the approximation properties of $Q_{N}^{G}$ and $I_{N}^{G}$. Compared to the Hermite-Galerkin spectral method of Section 9.1.1, we have that $(-\Delta)^{s} R_{n}^{\lambda}(x)$ is not as compact as for the Hermite polynomials, but can be easily approximated by a finite series of hypergeometric functions.

\subsubsection{Approximation by modified Chebyshev polynomials}

It is noteworthy that $|\boldsymbol{\xi}|^{2 s}$, the symbol of the Fourier transform of the fractional Laplacian, is non-separable so that direct extensions to higher dimensions of methods developed for the one-dimensional setting can be very complicated. To overcome this computational difficulty, Sheng et al. (2019) proposed a reformulation of the weak formulation (9.2) by the DunfordTaylor formula (see Theorem 4.1 of Bonito, Lei and Pasciak 2019, and also Section 3.3.1), and constructed a spectral-Galerkin method having Fourierlike bi-orthogonal mapped Chebyshev polynomials as basis functions. This method leads to a diagonalized system that can be efficiently solved via the fast Fourier transform (FFT), and the approach can be easily extended to higher-dimensional problems via tensor products of the basis functions.

For any $u, v \in H^{s}(\mathbb{R})$, by the Dunford-Taylor formula, we have (see $(3.15))$

$$
\begin{aligned}
& \frac{C_{d, s}}{2} \int_{\mathbb{R}^{d}} \int_{\mathbb{R}^{d}} \frac{(u(\boldsymbol{y})-u(\boldsymbol{x}))(v(\boldsymbol{y})-v(\boldsymbol{x}))}{|\boldsymbol{y}-\boldsymbol{x}|^{d+2 s}} \mathrm{~d} \boldsymbol{y} \mathrm{d} \boldsymbol{x} \\
& =\frac{2 \sin (\pi s)}{\pi} \int_{0}^{\infty} t^{1-2 s} \int_{\mathbb{R}^{d}}\left((-\Delta)\left(I-t^{2} \Delta\right)^{-1} u\right)(\boldsymbol{x}) v(\boldsymbol{x}) \mathrm{d} \boldsymbol{x} \mathrm{d} t,
\end{aligned}
$$

where $I$ denotes the identity operator. Letting $w(\boldsymbol{x}):=\left(I-t^{2} \Delta\right)^{-1} u(\boldsymbol{x})$, we can rewrite the weak formulation (9.2) as: seek $u \in H^{s}\left(\mathbb{R}^{d}\right)$ such that for all $v \in H^{s}\left(\mathbb{R}^{d}\right)$

$$
\mathcal{A}_{s}(u, v)=\frac{2 \sin (\pi s)}{\pi} \int_{0}^{\infty} t^{-1-2 s}(u-w, v) \mathrm{d} t+c(u, v)=(f, v),
$$

where $w=w(\boldsymbol{x} ; u, t) \in H^{1}\left(\mathbb{R}^{d}\right)$ is, for any $t>0$, the solution of

$$
t^{2}(\nabla w, \nabla \psi)+(w, \psi)=(u, \psi) \text { for all } \psi \in H^{1}\left(\mathbb{R}^{d}\right) .
$$


The spectral-Galerkin approximation to (9.7)-(9.8) is then given as follows: find $u_{N} \in X_{N}^{C}$ such that

$$
\begin{aligned}
\mathcal{A}_{s}\left(u_{N}, v_{N}\right) & =\frac{2 \sin (\pi s)}{\pi} \int_{0}^{\infty} t^{-1-2 s}\left(u_{N}-w_{N}, v_{N}\right) \mathrm{d} t+c\left(u_{N}, v_{N}\right) \\
& =\left(I_{N} f, v_{N}\right) \quad \text { for all } v_{N} \in X_{N}^{C},
\end{aligned}
$$

where $w_{N}\left(\boldsymbol{x} ; u_{N}, t\right) \in X_{N}^{C}$ is, for any $t>0$, the solution of

$$
t^{2}\left(\nabla w_{N}, \nabla \psi_{N}\right)+\left(w_{N}, \psi_{N}\right)=\left(u_{N}, \psi_{N}\right) \text { for all } \psi_{N} \in X_{N}^{C} \text {. }
$$

To solve the numerical scheme (9.9)-(9.10), Sheng et al. (2019) used a basis of Fourier-like mapped Chebyshev polynomials. Recall that the classical Chebyshev polynomials are defined by, for $n=0,1, \ldots$,

$$
T_{n}(y)=\cos (n \arccos (y)) \quad \text { with } y \in(-1,1) .
$$

Then we define the mapped Chebyshev polynomials by

$$
R_{n}(x):=\left(1+x^{2}\right)^{-1 / 2} T_{n}\left(\frac{x}{\sqrt{1+x^{2}}}\right), \quad x \in \mathbb{R} .
$$

It is well known that the mapped Chebyshev functions are orthonormal in $L^{2}(\mathbb{R})$ but are not orthogonal in $H^{1}\left(\mathbb{R}^{d}\right)$. Following the spirit of Shen and Wang (2009), in Sheng et al. (2019) the approximation space is defined by $X_{N}^{C}=\operatorname{span}\left\{R_{n}: n=0,1, \ldots, N\right\}$ for which a Fourier-like basis that is biorthogonal can be constructed, that is, the basis functions are orthogonal with respect to both the $L^{2}$ and $H^{1}$ inner products. As a result, (9.10) is diagonalizable, as is (9.9), and hence they can be efficiently solved by an FFT related to Chebyshev polynomials.

The integration in terms of $t$ in (9.9) can be evaluated exactly by using a sinc quadrature scheme (Bonito et al. 2019) whose computational cost is negligible compared with that of an FFT. Furthermore, this fast algorithm can be easily applied in higher-dimensional settings by using tensor products of the one-dimensional basis functions. The resulting complexity of solving (9.9) is then of $O\left(N(\log N)^{d}\right)$, where $N$ is the number of degrees of freedom. The relevant error estimate is similar to that in (9.6) with $\lambda=0$.

Remark 9.1. Although the solution to the fractional diffusion problem (9.1) has a simple and closed form in the Fourier domain, all existing spectral methods fail to achieve at an exponential convergence rate. This is because the solution generally decays at an algebraic rate at infinity, even if the source term is smooth and compactly supported. The proposed Hermite and the mapped rational/Chebyshev polynomials can only approximate exponentially decaying functions with spectral accuracy. How one designs a spectral method that accurately captures the algebraic decaying behaviour of the solution to (9.1) remains a largely open problem. 
All the estimates stated in this subsection are derived with respect to the energy norm, e.g. $H^{s}\left(\mathbb{R}^{d}\right)$. There is, of course, interest in obtaining higher approximation rates with respect to the $L^{2}\left(\mathbb{R}^{d}\right)$-norm. However, the approximation properties of orthogonal polynomials are derived in weighted Sobolev spaces, so the usual duality arguments are not directly applicable here. Thus optimal $L^{2}\left(\mathbb{R}^{d}\right)$-norm error estimates are still not well understood and warrant further investigation.

\subsection{Spectral-Galerkin methods in bounded domains}

Now we turn to fractional diffusion in a bounded domain. Owing to the low regularity of the solution of the underlying problems involving the fractional Laplacian (see e.g. Ervin, Heuer and Roop 2018, Grubb 2015b, Ros-Oton and Serra 2014), classical spectral methods cannot approximate the solution very well. In this section we focus on the particular fractional Poisson problem (3.5) with $g(\boldsymbol{x})=0$, that is, on the problem

$$
\begin{cases}(-\Delta)^{s} u=f & \text { for all } \boldsymbol{x} \in \Omega, \\ u=0 & \text { for all } \boldsymbol{x} \in \Omega_{\mathcal{I}_{\infty}}=\mathbb{R}^{d} \backslash \Omega,\end{cases}
$$

where $f(\boldsymbol{x})$ is a smooth function and $\Omega$ is a bounded domain with a smooth boundary.

Mao, Chen and Shen (2016) considered the one-dimensional setting with $\Omega=(-1,1)$ and $s \in(1 / 2,1)$. In this case, the fractional Laplacian is equivalent to the Riesz-fractional differential derivative that is defined by, for $u \in C_{c}^{\infty}(-1,1)$ and $x \in(0,1)$

$$
-D^{2 s} u(x):=-\frac{1}{2 \cos ((1-s) \pi)} \frac{\mathrm{d}^{2}}{\mathrm{~d} x^{2}} \int_{-1}^{1}|x-t|^{1-2 s} u(t) \mathrm{d} t=(-\Delta)^{s} u(x) .
$$

Then problem (9.12) is equivalent to the two-point boundary value problem: find the function $u$ satisfying

$$
\left\{\begin{array}{l}
-D^{2 s} u(x)=f(x) \text { for all } x \in(-1,1), \\
u(0)=u(1)=0
\end{array}\right.
$$

The discussion in Mao et al. (2016) is motivated by the observation that

$$
(-\Delta)^{s}\left\{\left(1-x^{2}\right)_{+}^{s} P_{n}^{s, s}(x)\right\}=\frac{\Gamma(2 s+n+1)}{\Gamma(n+1)} P_{n}^{s, s}(x),
$$

where $P_{n}^{a, b}$ with $a, b>-1$ denotes the classical Jacobi polynomials that are orthogonal with respect to the weight function $w^{a, b}(x)=(1-x)^{a}(1+x)^{b}$. This implies that if the source term $f(x)$ can be expanded in terms of the Jacobi polynomials $P_{n}^{s, s}(x)$, then the solution of the fractional diffusion problem (9.12) is a series in $\left(1-x^{2}\right)_{+}^{s} P_{n}^{s, s}(x)$. 
By choosing the test space $V_{N}=\operatorname{span}\left\{P_{n}^{s, s}(x): n=0, \ldots, N\right\}$ and the trial space $X_{N}=\operatorname{span}\left\{\left(1-x^{2}\right)_{+}^{s} P_{n}^{s, s}(x): n=0, \ldots, N\right\}$, a Petrov-Galerkin spectral method is given as follows: find $u_{N} \in X_{N}$ such that, for all $v_{N} \in$ $V_{N}$,

$$
\int_{-1}^{1}(-\Delta)^{s} u_{N}(x) v_{N}(x)\left(1-x^{2}\right)^{s} \mathrm{~d} x=\int_{-1}^{1} f(x) v_{N}(x)\left(1-x^{2}\right)^{s} \mathrm{~d} x .
$$

Using the relation (9.14) and the approximation property of Jacobi polynomials (Shen et al. 2011, Theorem 3.35), the following error estimate in the weighted $L^{2}$-norm is derived in Mao et al. (2016, Theorem 5):

$$
\left\|u_{N}-u\right\|_{B_{-s}^{0}} \leq C N^{-2 s-m}\|f\|_{B_{s}^{m}} \text { for any integer } m \geq 0
$$

where the norm $\|\cdot\|_{B_{\lambda}^{m}}$ is defined by

$$
\|v\|_{B_{\lambda}^{m}}^{2}=\sum_{j=0}^{m} \int_{-1}^{1}\left|v^{(j)}\right|^{2}\left(1-x^{2}\right)^{j+\lambda} \mathrm{d} x .
$$

If $m$ is not an integer, the space $B_{\lambda}^{m}$ is defined by interpolation (Guo and Wang 2004). The argument also applies to the case when $s \in(0,1 / 2]$ and $\Omega$ is multi-interval (Acosta, Borthagaray, Bruno and Maas 2018).

The above estimate indicates the exponential convergence under the assumption that the source term $f$ is smooth. The results in Mao et al. (2016) extend the studies of spectral methods for solving one-sided fractional equations found in Chen, Shen and Wang (2016b), Zayernouri and Karniadakis (2014) and Zayernouri, Ainsworth and Karniadakis (2015b); see also Mao and Karniadakis (2018) and Samiee, Zayernouri and Meerschaert $(2019 a, 2019 b)$ for general two-sided fractional equations, Zayernouri, Ainsworth and Karniadakis (2015a) for tempered fractional diffusion equations, and Deng, Zhang and Zhao (2019) for the study of superconvergence points.

A similar analysis may be extended to higher-dimensional cases. Xu and Darve $(2018 b)$ studied a spectral method for solving the fractional Poisson problem (9.13) with $\Omega=B_{1}(\mathbf{0}) \subset \mathbb{R}^{d}, d=2,3$, that is, the unit ball in two or three dimensions. The basic idea is to construct a spectral basis by using Jacobi polynomials and spherical harmonic polynomials (Dyda, Kuznetsov and Kwaśnicki 2017a). In particular, spherical harmonic polynomials of degree $\ell \geq 0$ form a finite-dimensional space, having dimension

$$
M_{d, \ell}=\frac{d+2 \ell-2}{d+\ell-2}\left(\begin{array}{c}
d+\ell-2 \\
\ell
\end{array}\right)
$$

For each $\ell$ we fix a linear basis for this space, denoted by $V_{\ell, m}$ with $m=$ $1, \ldots, M_{d, \ell}$, that is orthonormal with respect to the surface measure on the 
unit sphere. Then the functions

$\phi_{\ell, m, n}(\boldsymbol{x})=V_{\ell, m}(\boldsymbol{x}) P_{n}^{s, d / 2+\ell-1}\left(2|\boldsymbol{x}|^{2}-1\right) \quad$ with $\ell \geq 0, n \geq 0,0 \leq m \leq M_{d, \ell}$ form an orthogonal basis in $L_{w}^{2}(\Omega)$ with respect to the weight function $w(\boldsymbol{x})=\left(1-|\boldsymbol{x}|^{2}\right)^{s}$ (Dyda et al. 2017a). Analogous to (9.14), we have

$$
(-\Delta)^{s}\left(\left(1-|\boldsymbol{x}|^{2}\right)_{+}^{s} \phi_{\ell, m, n}\right)=\frac{2^{2 s} \Gamma(s+n+1) \Gamma(d / 2+\ell+s+n)}{n ! \Gamma(d / 2+n)} \phi_{\ell, m, n}(\boldsymbol{x}) .
$$

$\mathrm{Xu}$ and Darve (2018b) have developed and analysed fast and accurate spectral methods based on this property and the approximation property of the Jacobi polynomials.

Unfortunately, the above argument heavily relies on the relation (9.14) so that, due to the singular behaviour of the solution near the boundary, it cannot be directly applied to the fractional diffusion with lower-order terms. Zhang (2019) studied the regularity of the one-dimensional fractional diffusion-reaction model

$$
\begin{cases}(-\Delta)^{s} u(x)+c u(x)=f(x) & \text { for all } x \in(-1,1) \\ u(x)=0 & \text { for all } x \in \mathbb{R} \backslash(-1,1)\end{cases}
$$

with the constant $s \in(0,1)$ and $c>0$. In particular, Zhang proved that $\left(1-x^{2}\right)^{-s} u$ belongs to $B_{s}^{(2 s \wedge m)+2 s}$ if $f \in B_{s}^{m}$. Moreover, the regularity index could be slightly improved, that is,

$$
\left(1-x^{2}\right)^{-s} u \in B_{s-1}^{(6 s-\epsilon) \wedge(1+4 s)} \text { provided that } f \in B_{s-1}^{m} \text { for large } m .
$$

The convergence rate of the spectral method certainly deteriorates accordingly, due to a lack of sufficient solution regularity. See also Mao and Shen (2018) for a spectral element method based on geometric meshes.

Remark 9.2. In one dimension, the fractional Poisson problem without lower-order terms has been comprehensively studied. The analysis of the regularity and numerical approximations relies crucially on the simple relation (9.14), which indicates that the Jacobi polynomials are suitable to approximate the solution. This is largely the main reason why problems without lower-order terms dominate the study of spectral methods for fractional diffusion. Thus it is of significant interest to develop proper techniques for rigorously handling convection and reaction terms.

The numerical analysis of higher-dimensional fractional problems is a nontrivial endeavour. One reason is the difficulty of designing spectral methods for irregular domains. Another is due to the unclear regularity theory of (9.12) in higher-dimensional domains, even for convex polygonal domains. Therefore it is of substantial interest to analyse spectral methods for solving fractional diffusion problems in bounded domains in higher dimensions. Many theoretical questions, e.g. regularity in suitable spaces, appropriate 
selection of basis functions and optimal convergence rates, remain largely open problems.

\section{Finite difference methods for the strong form of nonlocal diffusion}

The AC property was first demonstrated for quadrature-based finite difference discretizations of nonlocal models in Tian and Du (2013). Additional observations can be found in Du and Tian (2015). Du, Tao, Tian and Yang (2016b) studied an AC quadrature difference discretization together with superconvergent nonlocal gradient recovery. Du et al. (2019c) considered quadrature rules for scalar models in multidimensional spaces, resulting in $\mathrm{AC}$ schemes. The key to obtaining AC non-variational methods in these works is to guarantee the uniform truncation errors of the numerical schemes independent of the parameter $\delta$. Numerical methods for the strong form of nonlocal diffusion are analysed under the standard framework of truncation error analysis and numerical stability. Quadrature-based finite difference schemes for nonlocal diffusion equation are introduced in Du et al. (2019c) and Tian and Du (2013), and a reproducing kernel (RK) collocation method is studied in Leng, Tian, Trask and Foster (2019). Note that although Trask, You, Yu and Parks (2019) refer to an AC mesh-free scheme, their numerical scheme converges only to the corresponding local solution but not to the nonlocal solution with a fixed $\delta$.

We denote a uniform Cartesian grid on $\mathbb{R}^{d}$ as

$$
\mathcal{T}_{h}:=\left\{\boldsymbol{x}_{\boldsymbol{j}}=h \boldsymbol{j}: \boldsymbol{j} \in \mathbb{Z}^{d}\right\} .
$$

We can then rewrite the nonlocal diffusion operator at any node $\boldsymbol{x}_{\boldsymbol{i}} \in \mathcal{T}_{h} \cap \Omega$ as

$$
\mathcal{L}_{\delta} u\left(\boldsymbol{x}_{\boldsymbol{i}}\right)=2 \int_{\mathcal{B}_{\delta}(\mathbf{0})} \frac{u\left(\boldsymbol{z}+\boldsymbol{x}_{\boldsymbol{i}}\right)-u\left(\boldsymbol{x}_{\boldsymbol{i}}\right)}{W(\boldsymbol{z})} W(\boldsymbol{z}) \gamma_{\delta}(|\boldsymbol{z}|) \mathrm{d} \boldsymbol{z},
$$

where $W(\boldsymbol{z})$ is a weight function that is crucial to guaranteeing the AC property. One-dimensional problems are considered in Tian and Du (2013) with the weight function $W(z)=|z|$ used for $z \in \mathbb{R}$. In the multi-dimensional case considered in Du et al. $(2019 c)$, the weight function $W(\boldsymbol{z})=|\boldsymbol{z}|^{2} /|\boldsymbol{z}|_{1}$ is chosen, where $|\cdot|_{1}$ denotes the $\ell_{1}$-norm in the $d$-dimensional vector space whereas $|\cdot|$ denotes the standard Euclidean norm. The finite difference scheme on the Cartesian grid is then given by

$$
\mathcal{L}_{\delta, h} u\left(\boldsymbol{x}_{\boldsymbol{i}}\right)=2 \int_{\mathcal{B}_{\delta}(\mathbf{0})} \mathcal{I}_{h}\left(\frac{\left(u\left(\boldsymbol{z}+\boldsymbol{x}_{\boldsymbol{i}}\right)-u\left(\boldsymbol{x}_{\boldsymbol{i}}\right)\right.}{W(\boldsymbol{z})}\right) W(\boldsymbol{z}) \gamma_{\delta}(|\boldsymbol{z}|) \mathrm{d} \boldsymbol{z},
$$

where $\mathcal{I}_{h}(\cdot)$ denotes the piecewise $d$-multilinear interpolation operator in $\boldsymbol{z}$ associated with the grid $\mathcal{T}_{h}$. 
There are two major features of the finite difference scheme (10.1). The first is a uniform consistency result. In this regard, the following result about what we refer to as quadratic exactness is proved in Du et al. (2019c); it is a nonlocal analogue of its local counterpart. That is, the fact that the centred difference approximation to the Laplacian is exact for quadratic polynomials holds in the nonlocal case as well.

Quadratic exactness. For any quadratic polynomial in $\mathbb{R}^{d}$ given as $u(\boldsymbol{x})=$ $\boldsymbol{x} \otimes \boldsymbol{x}: M$, where $M=\left(m_{k j}\right)$ denotes a constant matrix and $\otimes$ denotes the tensor product, we have

$$
\mathcal{L}_{\delta, h} u\left(\boldsymbol{x}_{\boldsymbol{i}}\right)=\mathcal{L}_{\delta} u\left(\boldsymbol{x}_{\boldsymbol{i}}\right)=\sum_{k} m_{k k} \quad \text { for all } \boldsymbol{i} .
$$

Quadratic exactness plays a vital role in the analysis of the AC property of quadrature-based finite difference schemes. It leads to the uniform consistency result, which says that (10.1) is an $O\left(h^{2}\right)$ approximation of the nonlocal diffusion operator independent of the parameter $\delta$, which means that the truncation error is independent of $\delta$ for small $\delta$.

Uniform truncation error. Assume that $u \in C^{4}\left(\overline{\Omega_{\delta}}\right)$. Then, for all $\boldsymbol{x}_{\boldsymbol{i}} \in$ $\mathcal{T}_{h} \cap \Omega$, we have

$$
\left|\mathcal{L}_{\delta, h} u\left(\boldsymbol{x}_{\boldsymbol{i}}\right)-\mathcal{L}_{\delta} u\left(\boldsymbol{x}_{\boldsymbol{i}}\right)\right| \leq C\left|D^{4} u\right|_{\infty} h^{2},
$$

where $C$ denotes a constant independent of $\delta$ and $h$.

With this observation, it then follows that $u_{\delta, h}$ approximates $u_{0}$ at the rate $O\left(h^{2}+\delta^{2}\right)$, once numerical stability is established.

Another major feature of (10.1) is that it satisfies a discrete maximum principle, thus it is a stable numerical scheme. In fact, (10.1) can be rewritten as

$$
\mathcal{L}_{\delta, h} u\left(\boldsymbol{x}_{\boldsymbol{i}}\right)=\sum_{\boldsymbol{x}_{\boldsymbol{j}} \in B_{\delta}\left(\boldsymbol{x}_{\boldsymbol{i}}\right)} a_{\boldsymbol{i}, \boldsymbol{j}}\left(u\left(\boldsymbol{x}_{\boldsymbol{j}}\right)-u\left(\boldsymbol{x}_{\boldsymbol{i}}\right)\right),
$$

where each $a_{i, j}$ is a nonnegative number given by

$$
a_{i, \boldsymbol{j}}=\frac{2}{W(h(\boldsymbol{j}-\boldsymbol{i}))} \int_{\mathcal{B}_{\delta}(\mathbf{0})} \varphi_{\boldsymbol{j}}(\boldsymbol{z}+h \boldsymbol{i}) W(\boldsymbol{z}) \gamma_{\delta}(|\boldsymbol{z}|) \mathrm{d} \boldsymbol{z},
$$

with $\varphi_{\boldsymbol{j}}$ being the piecewise multilinear basis function centred at $\boldsymbol{x}_{\boldsymbol{j}}=h \boldsymbol{j}$; $\varphi_{\boldsymbol{j}}$ satisfies $\varphi_{\boldsymbol{j}}\left(\boldsymbol{x}_{\boldsymbol{i}}\right)=0$ when $\boldsymbol{i} \neq \boldsymbol{j}$ and $\varphi_{\boldsymbol{j}}\left(\boldsymbol{x}_{\boldsymbol{j}}\right)=1$. The discrete maximum principle of (10.4) can then be easily seen from the fact that $a_{i, j} \geq 0$ for each $\boldsymbol{i}$ and $\boldsymbol{j}$.

Equation (10.4) gives a second-order accurate $\mathrm{AC}$ finite difference scheme on uniform Cartesian grids. Higher-order finite difference schemes may also be constructed. However, the discrete maximum principle is not satisfied 
for higher-order methods because $a_{\boldsymbol{i}, \boldsymbol{j}}$ could be negative for higher-order interpolants. In this case, one needs new techniques to study the stability of numerical schemes, which is still an open problem except for some specialized kernels; see Leng et al. (2019).

We note that there have been other works concerning AC schemes for nonlocal models based on the strong form. For example, Du, Zhang and Zheng (2018d), You, Yu and Kamensky (2019) and Tao et al. (2019) have studied AC schemes for coupled local and nonlocal models. Du, Han, Zheng and Zhang (2018a), Zhang, Yang, Zhang and Du (2017) and Du et al. $(2018 d)$ presented implementation techniques and numerical experiments of $\mathrm{AC}$ schemes for problems defined in infinite domains via the development of nonlocal artificial boundary conditions. In these works, time-dependent nonlocal models are considered. Similar studies have also been made for models that are nonlocal in time and space (Chen, Du, Li and Zhou 2017), which are generalizations of models developed in Du, Yang and Zhou (2017b) and Du, Toniazzi and Zhou (2020b). Du et al. (2017b, 2020b) only considered nonlocal memory/history effects in time but the spatial interactions remained local. Chen et al. (2017) also replaced the local spatial differential operators with nonlocal operators.

$\mathrm{AC}$ difference approximations of nonlinear models have also been investigated, including approximations of nonlocal hyperbolic conservation laws. From a modelling perspective, one can argue that conservation laws in integral forms may be particularly more natural than their local counterpart, especially in the presence of singular solution behaviours. Important physics might be lost in the local formulation, so additional assumptions such as entropy conditions have to be re-introduced to maintain validity. On the other hand, it is possible to introduce nonlocal conservation laws for which appropriate entropy conditions are automatically satisfied (Du, Huang and LeFloch $2017 a$ ), thus retaining important physical features in the modelling process that are missing from local models. The model of Du et al. (2017a) also improves the model studied by Du, Kamm, Lehoucq and Parks (2012b). With AC discretization (Du and Huang 2017), the numerical convergence has been demonstrated with or without singular solutions.

We also mention some other works concerning difference approximations of nonlocal models. For example, Jha and Lipton (2019) studied the discretization of peridynamic models involving bond-softening. Regarding the consistency between the approximations of nonlocal models and their local limits, one can also find comparisons in Bobaru et al. (2009) and Seleson et al. (2016).

Leng et al. (2019) discretized the strong form of nonlocal diffusion operators by using the reproducing kernel particle method (RKPM) (Liu, Jun and Zhang 1995). The work shows the consistency and stability of the linear RK collocation scheme on quasi-uniform Cartesian grids. Define a 
quasi-uniform Cartesian grid on $\mathbb{R}^{d}$ as

$$
\mathcal{T}_{\boldsymbol{h}}=\left\{\boldsymbol{x}_{\boldsymbol{j}}:=\boldsymbol{h} \odot \boldsymbol{j}: \boldsymbol{j} \in \mathbb{Z}^{d}\right\},
$$

where $\boldsymbol{h}$ denotes a vector in $\mathbb{R}^{d}$ and $\odot$ denotes component-wise multiplication of vectors. We also assume that $\boldsymbol{h}=h \overline{\boldsymbol{h}}$, where $h \in \mathbb{R}$ and $\overline{\boldsymbol{h}}$ is a fixed unit vector in $\mathbb{R}^{d}$, so that the convergence rate will again be given in terms of $h \in \mathbb{R}$. RKPM provides a systematic means for generating basis functions from scattered particles such that polynomials can be exactly represented up to a certain order. Here we assume that the RK basis functions $\left\{\Psi_{\boldsymbol{j}}(\boldsymbol{x})\right\}_{\boldsymbol{j} \in \mathbb{Z}^{d}}$ are generated with respect to the quasi-uniform Cartesian grid $\mathcal{T}_{\boldsymbol{h}}$. Then the RK interpolation of any continuous function $u$ is defined as

$$
\left(\mathcal{R}_{h} u\right)(\boldsymbol{x}):=\sum_{\boldsymbol{j} \in \mathbb{Z}^{d}} \Psi_{\boldsymbol{j}}(\boldsymbol{x}) u\left(\boldsymbol{x}_{\boldsymbol{j}}\right) .
$$

Leng et al. (2019) considered the RK basis functions given by

$$
\Psi_{\boldsymbol{j}}(\boldsymbol{x}):=\prod_{k=1}^{d} \varphi\left(\frac{[\boldsymbol{x}]_{k}-\left[\boldsymbol{x}_{\boldsymbol{j}}\right]_{k}}{[\boldsymbol{a}]_{k}}\right),
$$

where $[\boldsymbol{x}]_{k}$ denotes the $k$ th component of the vector $\boldsymbol{x} \in \mathbb{R}^{d}$. The vector $\boldsymbol{a} \in \mathbb{R}^{d}$ is assumed to satisfy $\boldsymbol{a}=m \boldsymbol{h}$ with $m>0$ being an even number. The function $\varphi$ is the cubic B-spline given by

$$
\varphi(x)= \begin{cases}\frac{2}{3}-4|x|^{2}+4|x|^{3} & 0 \leq|x| \leq \frac{1}{2}, \\ \frac{4}{3}(1-|x|)^{3} & \frac{1}{2} \leq|x| \leq 1, \\ 0 & \text { otherwise. }\end{cases}
$$

Those assumptions allow the RK approximation $\mathcal{R}_{h} u$ to represent multilinear polynomials exactly, and a special synchronized convergence property is satisfied (Li and Liu 1996).

Given the RK interpolation operator $\mathcal{R}_{h}$, the RK collocation scheme is then defined as

$$
\mathcal{L}_{\delta, h} u\left(\boldsymbol{x}_{\boldsymbol{i}}\right)=\mathcal{L}_{\delta}\left(\mathcal{R}_{h} u\right)\left(\boldsymbol{x}_{\boldsymbol{i}}\right),
$$

for any $\boldsymbol{x}_{\boldsymbol{i}} \in \mathcal{T}_{\boldsymbol{h}} \cap \Omega$. One nice property about the interpolation operator $\mathcal{R}_{h}$ defined above is that although it only reproduces multilinear polynomials exactly, it shifts quadratic polynomials by a constant, so that $\mathcal{L}_{\delta, h}$ defined in (10.7) actually satisfies the quadratic exactness result given earlier. This property and the synchronized convergence property become the crucial reasons that cause the scheme (10.7) to be AC. Indeed, Leng et al. (2019) showed that the collocation scheme (10.7) satisfies the same uniform consistency result presented above. The stability of the scheme, however, is 
a more tricky issue. In fact, all RK collocation schemes fail to satisfy the discrete maximum principle. But the linear RK basis function $\Psi_{\mathbf{0}}$ given by (10.6) has a special property that its Fourier transform, given by

$$
\widehat{\Psi_{\mathbf{0}}}(\boldsymbol{\xi})=\prod_{k=1}^{d}[\boldsymbol{a}]_{k} \cdot \widehat{\varphi}\left([\boldsymbol{a}]_{k}[\boldsymbol{\xi}]_{k}\right)=\prod_{k=1}^{d} \frac{[\boldsymbol{a}]_{k}}{2}\left(\frac{\sin \left([\boldsymbol{a}]_{k}[\boldsymbol{\xi}]_{k} / 4\right)}{\left([\boldsymbol{a}]_{k}[\boldsymbol{\xi}]_{k} / 4\right)}\right)^{4}
$$

is always nonnegative. This result is a key observation used by Leng et al. (2019) to show that the strong form discretization (10.7) is comparable in terms of Fourier symbols with the Galerkin approximation (5.5), with $V_{\delta, h}$ being the span of RK basis functions (10.6). This immediately implies the stability of the scheme (10.7), because the standard Galerkin approximation is naturally stable. Stability issues again prevent us from discussing higherorder RK collocation methods because the higher-order RK basis functions fail to have purely nonnegative Fourier transforms such as one shown in (10.8). More careful investigations are needed for the stability analysis of higher-order methods. As a last comment, we note that Leng, Tian, Trask and Foster (2020) have extended the analysis of Leng et al. (2019) to the peridynamics Navier system.

\section{Numerical methods for the strong form of fractional diffusion}

In contrast to Sections 6,7 and 9 , in which we studied the discretization of a weak formulation of a fractional diffusion model, in this section we consider discretizations of a strong formulation. We consider three such approaches: quadrature rule-based finite difference methods, Monte Carlo methods and radial basis function methods.

\subsection{Quadrature rule-based finite difference methods}

The most used class of methods for discretizing fractional diffusion models in bounded domains is quadrature rule-based finite difference methods, where their popularity stems from their simplicity. These methods directly discretize the integral fractional Laplacian that features a singular, nonintegrable integrand. A common approach to dealing with the difficulties that singular integrals pose is to split the integral into the sum of two integrals, one isolating the singular part, for which some care should be exercised, and the other having a smooth integrand so that standard quadrature rules can be used to obtain accurate approximations. This approach has been widely used for solving the nonlocal diffusion models (Du et al. 2019c, Tian and Du 2013), as reported in Section 10. In the same spirit, some variants were also developed for fractional diffusion models. 
We have the one-dimensional fractional equation and volume constraint

$$
\begin{cases}(-\Delta)^{s} u(x)=C_{1, s} \int_{-\infty}^{\infty} \frac{u(x)-u(x+z)}{|z|^{1+2 s}} \mathrm{~d} z=f(x) & \text { for all } x \in \Omega, \\ u(x)=0 & \text { for all } x \in \Omega_{\mathcal{I}_{\infty}},\end{cases}
$$

with $s \in(0,1), \Omega=(0, L)$ with $L>0$, and $\Omega_{\mathcal{I}_{\infty}}=\mathbb{R} \backslash \Omega$. To discretize the problem, we use the uniform Cartesian grid $\left\{x_{j}=j h: j \in \mathbb{Z}\right\}$ with $h=L / N$ for some integer $N$. In Huang and Oberman (2014), the fractional equation in (11.1) is evaluated at the grid points $x_{j}$ and then split into two integrals, that is, for $j=0, \ldots, N$, we have

$$
\begin{aligned}
(-\Delta)^{s} u\left(x_{j}\right)= & \underbrace{C_{1, s} \int_{|z|<h} \frac{u\left(x_{j}\right)-u\left(x_{j}+z\right)}{|z|^{1+2 s}} \mathrm{~d} z}_{I_{1}} \\
& +\underbrace{C_{1, s} \int_{|z|>h} \frac{u\left(x_{j}\right)-u\left(x_{j}+z\right)}{|z|^{1+2 s}} \mathrm{~d} z}_{I_{2}} .
\end{aligned}
$$

For smooth $u$ the use of Taylor series and central difference scheme leads to

$$
I_{1}=-\frac{C_{1, s}}{2-2 s}\left(\frac{u\left(x_{j+1}\right)-2 u\left(x_{j}\right)+u\left(x_{j-1}\right)}{h^{2 s}}\right)+O\left(h^{4-2 s}\right) .
$$

The second term $I_{2}$ in (11.2) has a regular integrand so that term can be well approximated using standard quadrature rules. As an example, let $I_{h}^{k}$ denote the Lagrange interpolation operator for piecewise polynomials of order less than or equal to $k$. Then, for smooth $u$,

$$
I_{2}=C_{1, s} \int_{|z|>h} \frac{\mathcal{I}_{h}^{k}\left(u\left(x_{j}\right)-u\left(x_{j}+z\right)\right)}{|z|^{1+2 s}} \mathrm{~d} z+O\left(h^{k+1-2 s}\right) .
$$

Then (11.3) and (11.4) result in a discrete approximation of the fractional Laplacian:

$$
(-\Delta)^{s} u\left(x_{j}\right)=D_{h} u\left(x_{j}\right)+O\left(h^{\min (4, k+1)-2 s}\right),
$$

where the discrete operator $D_{h}$ is defined by

$$
\begin{aligned}
D_{h} u\left(x_{j}\right):= & \frac{C_{1, s}}{2-2 s} \frac{-u\left(x_{j-1}\right)+2 u\left(x_{j}\right)-u\left(x_{j+1}\right)}{h^{2 s}} \\
& +C_{1, s} \int_{|z|>h} \frac{\mathcal{I}_{h}^{k}\left(u\left(x_{j}\right)-u\left(x_{j}+z\right)\right)}{|z|^{1+2 s}} \mathrm{~d} z .
\end{aligned}
$$

As a result, the corresponding quadrature-based finite difference scheme is 
given as follows: find $U_{j}, j \in \mathbb{A}=\{1, \ldots, N-1\}$, such that

$$
D_{h} U_{j}=f\left(x_{j}\right) \quad \text { with } U_{j}=0 \text { for all } j \in \mathbb{Z} \backslash \mathbb{A} .
$$

Similar to discussions given in Section 10, a maximum principle for the above scheme (and hence its stability) can be proved (see Huang and Oberman 2014 for $k=1$ and 2), by using a barrier function method, and therefore the error estimate follows immediately. Del Teso, Endal and Jakobsen $(2018,2019)$ extended the aforementioned approach to higher-dimensional problems, and improved the convergence rate by using adapted vanishing viscosity approximation.

Minden and Ying (2018) discussed the discretization in two and three dimensions by applying the 'window' function $w(z):=w(|z|)$ such that $1-w(z)=O\left(|z|^{p}\right)$ as $z \rightarrow 0$ for some positive integer $p$. Then the following splitting is applied:

$$
\begin{aligned}
(-\Delta)^{s} u\left(x_{j}\right) & \\
= & C_{d, s} \int_{\mathbb{R}^{d}} \frac{u(x)-u(y)+w(|x-y|) \sum_{1 \leq|\beta| \leq 3} D^{\beta} u(x)(y-x)^{\beta} /|\beta| !}{|x-y|^{d+2 s}} \mathrm{~d} y \\
& -C_{d, s} \int_{\mathbb{R}^{d}} \frac{w(|x-y|) \sum_{1 \leq|\beta| \leq 3} D^{\beta} u(x)(y-x)^{\beta} /|\beta| !}{|x-y|^{d+2 s}} \mathrm{~d} y .
\end{aligned}
$$

Note that the second term is regular and can thus be easily approximated by standard quadrature rules. On the other hand, by using a window function, the first term can be evaluated by the trapezoidal rule. If the grid is uniform, then the resulting scheme provides a discrete operator that is translation-invariant and therefore an FFT can be used (Minden and Ying 2018, Section 3) for an efficient solution.

Duo, van Wyk and Zhang (2018) and Duo and Zhang (2019) suggested another splitting approach, namely

$$
\frac{u(x)-u(y)}{|x-y|^{d+2 s}}=\left(|x-y|^{-d+\beta-2 s}\right)\left((u(x)-u(y))|x-y|^{-\beta}\right)
$$

for an appropriate parameter $\beta \in(2 s, 2)$. The first term is singular but can be evaluated analytically, whereas the second term is regular so it can be approximated using the trapezoidal rule, for example.

The discrete systems obtained by the quadrature-based finite difference methods introduced above can be Toeplitz-like, which can be tackled by using an FFT, but only for shift-invariant discretizations that usually require the use of uniform grids. As we mentioned in Section 3.3.3, the solution of the fractional Poisson equation (11.1) is weakly singular near the boundary, and hence graded meshes are preferred. In this case, discretization results in dense and unstructured matrices, and hence the storage complexity will be $O\left(N^{2}\right)$ whereas the computational complexity will be $O\left(N^{3}\right)$. 
One promising strategy for efficiently solving the nonlocal problem (11.1) with unstructured meshes is to apply hierarchical matrices so that only linear storage and computational complexity are required; see $\mathrm{Xu}$ and Darve (2018a).

In one dimension, problem (11.1) is closely related to the fractional boundary problem involving the Riemann-Liouville fractional derivative or Riesz derivative (Podlubny 1999, Ortigueira 2006). For such problems, many finite difference methods have been studied (Meerschaert and Tadjeran 2004, Tadjeran, Meerschaert and Scheffler 2006, Çelik and Duman 2012, Meerschaert and Tadjeran 2006, Chen and Deng 2014, Ding, Li and Chen 2015) and fast algorithms (Pan, Ng and Wang 2016, Wang and Basu 2012, Wang and Du 2013, Wang and Du 2014, Zhang, Sun and Pang 2015, Lei and Sun 2013). See also the comprehensive survey by Li and Chen (2018) and the monograph by Karniadakis (2019) for details about these kinds of models and their numerical approximation.

\subsection{Monte Carlo method by Feynman-Kac formula}

Since its formulation in Kac (1949), the Feynman-Kac formula has been a powerful tool for both theoretical reformulations and practical simulations of local PDEs. The link it establishes between PDEs and related stochastic processes can be exploited to develop Monte Carlo methods that are effective, especially for the numerical simulation of high-dimensional problems (Curtiss 1950, Muller 1956, Sabelfeld and Simonov 1994, Yan, Cai and Zeng 2013).

Kyprianou, Osojnik and Shardlow (2018) have developed a Monte Carlo algorithm for approximately solving the fractional Poisson problem with inhomogeneous volume constraints given by

$$
\begin{cases}(-\Delta)^{s} u(\boldsymbol{x})=f(\boldsymbol{x}) & \text { for all } \boldsymbol{x} \in \Omega \\ u(\boldsymbol{x})=g(\boldsymbol{x}) & \text { for all } \boldsymbol{x} \in \Omega_{\mathcal{I}_{\infty}} .\end{cases}
$$

The solution representation of (11.8) has been extensively studied from both the analytical and probabilistic perspectives ${ }^{4}$; see e.g. Bogdan and Byczkowski (1999), Bucur (2016), Ros-Oton (2016) and Kyprianou et al. (2018). In particular, we assume that $\Omega$ is bounded, that $f \in C^{2 s+\epsilon}(\bar{\Omega})$ for some $\epsilon>0$, and that $g$ is continuous function belonging to $L_{2 s}^{1}\left(\mathbb{R}^{d} \backslash \Omega\right)$,

4 The connection between stochastic processes and fractional equations has been extensively investigated in, e.g., Meerschaert and Sikorskii (2012). In contrast, Burch, D'Elia and Lehoucq (2014), Burch and Lehoucq (2015) and D'Elia, Du, Gunzburger and Lehoucq (2017) discuss the connection between finite-range jump processes and nonlocal (truncated) equations. 
that is,

$$
\int_{\mathbb{R}^{d} \backslash \Omega} \frac{|g(\boldsymbol{x})|}{1+|\boldsymbol{x}|^{2 s+d}} \mathrm{~d} x<\infty .
$$

Then the solution of the fractional Poisson problem (11.8) has the probabilistic representation (Kyprianou et al. 2018, Theorem 6.1)

$$
u(\boldsymbol{x})=\mathbb{E}_{\boldsymbol{x}}\left[g\left(X_{\sigma_{\Omega}}\right)\right]+\mathbb{E}_{\boldsymbol{x}}\left[\int_{0}^{\sigma_{\Omega}} f\left(X_{s}\right) \mathrm{d} s\right],
$$

with $\mathbb{E}_{\boldsymbol{x}}(\cdot)$ denoting the expected value conditioned on $X_{0}=\boldsymbol{x}$ and where $X=\left(X_{t}, t \geq 0\right)$ is an isotropic $\alpha$-stable Lévy process with index $\alpha=2 s$ and $\sigma_{\Omega}=\inf \left\{t>0: X_{t} \notin \Omega\right\}$ is the first exit time of $X_{t}$ from $\Omega$. In particular, let $B(0,1) \subset \mathbb{R}^{d}$ be a unit ball centred at the origin, and then for $|\boldsymbol{y}|>1$ we have (Blumenthal, Getoor and Ray 1961, Theorem A)

$$
\mathbb{P}_{0}\left(X_{\sigma_{B(0,1)}} \in \mathrm{d} \boldsymbol{y}\right)=\left.\left.\frac{\Gamma(d / 2) \sin (\pi \alpha / 2)}{\pi^{d / 2+1}}|1-| \boldsymbol{y}\right|^{2}\right|^{-\alpha / 2}|\boldsymbol{y}|^{-d} \mathrm{~d} \boldsymbol{y} .
$$

This gives the distribution of the stable process that begins from the origin when it first exits the unit ball.

The formula (11.9) is a nonlocal analogue to the Feynman-Kac formula of the classical Laplacian, but the role of $d$-dimensional Brownian motion is replaced by an isotropic $\alpha$-stable Lévy process in $\mathbb{R}^{d}$. One significant difference to Brownian motion is that the $\alpha$-stable Lévy process will exit the domain by a jump rather than hitting the boundary.

Then the solution to (11.8) can be computed numerically by applying a Monte Carlo sampling method, based on the Feynman-Kac formula (11.9). That is to say, if $\left(X_{t}^{i}, t \leq \sigma_{\Omega}^{i}\right)$ are independent and identically distributed copies of $\left(X_{t}, t \leq \sigma_{\Omega}\right)$ issued from $\boldsymbol{x} \in \Omega$, then by the law of large numbers, we obtain

$$
u(\boldsymbol{x})=\lim _{n \rightarrow \infty} \frac{1}{n} \sum_{i=1}^{n}\left(g\left(X_{\sigma_{\Omega}^{i}}^{i}\right)+\int_{0}^{\sigma_{\Omega}^{i}} f\left(X_{s}^{i}\right) \mathrm{d} s\right),
$$

where sample paths of the stable process could be constructed by (11.10). However, the direct application of a Monte Carlo method based on the Feynman-Kac formula is inefficient, because the evaluation of $u(\boldsymbol{x})$ requires the simulation of a very large number of paths of $X_{t}$, beginning from the single point $\boldsymbol{x} \in \Omega$.

One way to speed things up is to apply the walk-on-spheres strategy (WOS), that was first established in Muller (1956) for the Laplace equation and then adopted in Kyprianou et al. (2018) for the fractional Laplace equation. The WOS method does not require a complete simulation of 
the entire path and takes advantage of the distributional symmetry of the stochastic process $X_{t}$. Next we briefly discuss the WOS method for solving the fractional diffusion problem (11.8) within a convex domain $\Omega \subset \mathbb{R}^{d}$, $d \geq 2$.

With an arbitrary $\boldsymbol{x} \in \Omega$, we let $\boldsymbol{\rho}_{0}=\boldsymbol{x}$ and define $r_{1}$ to be the radius of the largest sphere inscribed in $\Omega$ that is centred at $\boldsymbol{\rho}_{0}$. Then we set $B_{1}=\left\{\boldsymbol{x} \in \mathbb{R}^{d}:\left|\boldsymbol{x}-\boldsymbol{\rho}_{0}\right|<r_{1}\right\}$ and select $\boldsymbol{\rho}_{1}$ according to $X_{\sigma_{B_{1}}}$ under $\mathbb{P}_{\boldsymbol{\rho}_{0}}$, which is known from (11.10). Repeating the argument, we construct a sequence $\left\{\boldsymbol{\rho}_{n}\right\}_{n \geq 1}$ inductively. The algorithm ends at the random index $N=\min \left\{n \geq 0: \boldsymbol{\rho}_{n} \notin \Omega\right\}$. As a result, the Feynman-Kac formula (11.11) can be replaced by (Kyprianou et al. 2018, Corollary 6.4)

$$
u(\boldsymbol{x})=\mathbb{E}_{\boldsymbol{x}}\left[g\left(\boldsymbol{\rho}_{N}\right)\right]+\mathbb{E}_{\boldsymbol{x}}\left[\sum_{n=0}^{N-1} r_{n}^{\alpha} \int_{|\boldsymbol{y}|<1} f\left(\boldsymbol{\rho}_{n}+r_{n} \boldsymbol{y}\right) V_{1}(0, \mathrm{~d} \boldsymbol{y})\right],
$$

where $V_{1}(0, \mathrm{~d} \boldsymbol{y})$ denotes the expected occupation measure of the stable process prior to exiting a unit ball centred at the origin, which is given for $|\boldsymbol{y}|<1$ by (Blumenthal et al. 1961, Corollary 4)

$$
V_{1}(0, \mathrm{~d} \boldsymbol{y})=2^{-\alpha} \pi^{-d / 2} \frac{\Gamma(d / 2)}{\Gamma(s)^{2}}|\boldsymbol{y}|^{2 s-d}\left(\int_{0}^{|\boldsymbol{y}|^{-2}-1}(u+1)^{-d / 2} u^{s-1} \mathrm{~d} u\right) \mathrm{d} \boldsymbol{y} .
$$

We refer interested readers to Kyprianou et al. (2018) for more details about convergence analysis, discussion of nonconvex domain and implementation of WOS.

A multilevel Monte Carlo method based on the WOS algorithm was proposed by Shardlow (2019) for efficiently computing the solution for the entire domain $\Omega$. Shardlow (2019, Section 5) drew comparisons between the multilevel WOS Monte Carlo method and the adaptive finite element method of Ainsworth and Glusa (2017).

\subsection{Radial basis function methods}

The development, analysis and implementation of mesh-free methods using radial basis functions (RBFs) has been studied thoroughly for the integer order (local) PDEs; see Buhmann $(2000,2003)$ and the references cited therein. Compared to finite difference methods, the RBF method is seemingly easier to implement for approximately solving the nonlocal problem (11.1), especially in high dimensions, because only minor modifications to existing algorithms for local PDEs are needed.

However, studies of RBF methods for the fractional diffusion model are fairly scarce. Rosenfeld, Rosenfeld and Dixon (2019) used RBF interpolants to approximate the fractional Laplacian of a given function through a mesh- 
free pseudo-spectral method. Specifically, they used the compactly supported Wendland functions $\Phi(\boldsymbol{x})=\phi_{d, k}(|\boldsymbol{x}|)$ defined in Wendland (1995, 2005), where $k$ is a smoothness parameter. With the collection of points

$$
X=\left\{\boldsymbol{x}_{1}, \boldsymbol{x}_{2} \ldots, \boldsymbol{x}_{m}\right\} \subset \Omega,
$$

the mesh size $h$ of $X$ is defined by

$$
h_{X}=\sup _{\boldsymbol{x} \in \Omega} \min _{\boldsymbol{x}_{j} \in X}\left\|\boldsymbol{x}-\boldsymbol{x}_{j}\right\|_{2} .
$$

Then, for any function $f \in N_{\Phi}\left(\mathbb{R}^{d}\right)$, where $N_{\phi}\left(\mathbb{R}^{d}\right)$ denotes the native space of the RBFs (Wendland 2005), the interpolant of $f$ is defined by

$$
I_{X} f(\boldsymbol{x})=\sum_{j=1}^{n} w_{j} \Phi\left(\boldsymbol{x}-\boldsymbol{x}_{j}\right) \quad \text { such that } I_{X} f(\boldsymbol{z})=f(\boldsymbol{z}) \text { for all } \boldsymbol{z} \in X .
$$

Suppose that $\Phi \in C^{2 k}\left(\mathbb{R}^{d}\right)$ is symmetric and strictly positive definite, and $f \in N_{\Phi}\left(\mathbb{R}^{d}\right)$ is compactly supported. Then, by estimating the inverse Fourier transform, the following interpolation error result is proved in Rosenfeld et al. (2019, Proposition 3.2):

$$
\left|(-\Delta)^{s}\left(I_{X} f\right)(x)-(-\Delta)^{s} f(x)\right| \leq\left(c h_{X}^{k-2 \beta}\|f\|_{N_{\phi}\left(\mathbb{R}^{d}\right)}+E\right),
$$

where the parameter $\beta \in \mathbb{N}$ satisfies $2 \beta<k$ and $2 \beta-2 s>n$. Here $E$ denotes the residual error that can be further bounded by applying a smooth cutoff function (Rosenfeld et al. 2019, Theorem 3.1). Numerical experiments showed no measurable difference in the resulting estimations when using the cut-off function, compared to when the cut-off function is not used (Rosenfeld et al. 2019, Section 4.1). However, the optimal convergence rate of the method without cut-off functions remains an open question.

The above interpolation property inspires the future study of collocation methods (or Galerkin methods) using RBFs for approximately solving the fractional diffusion problem (11.1). Many interesting questions, for example the selection of suitable RBFs, the stability of numerical methods, optimal convergence rates and adaptive algorithms, are largely open and warrant further investigation. In addition, for high-dimensional problems, an additional challenge stems from the computation of the fractional Laplacian of the basis functions, which requires fast and accurate numerical approximation. See also Lehoucq and Rowe (2016) and Lehoucq, Narcowich, Rowe and Ward (2018) for a Galerkin method using RBFs for solving nonlocal diffusion with kernel functions that are both radial and integrable, and Pang, Chen and $\mathrm{Fu}$ (2015) and Sun et al. (2017) for collocation methods using RBFs for solving fractional diffusion with Riemann-Liouville-type fractional derivatives. 


\section{Conditioning and fast solvers}

The effectiveness of nonlocal modelling and simulations relies on the effective solution of the algebraic systems resulting from the discretization of nonlocal models. Thus a good theoretical understanding of the conditioning of the stiffness matrices for nonlocal problems is important. Results in this direction have been provided, using the Fourier analysis of the point spectrum for nonlocal operators, as given in Zhou and Du (2010); see also Aksoylu and Mengesha (2010), Aksoylu and Parks (2011), Aksoylu and Unlu (2014), Du et al. (2012a) and Seleson, Parks, Gunzburger and Lehoucq (2009) for additional discussions.

It is well known that a typical local diffusion model yields a condition number of $O\left(h^{-2}\right)$ for a discretization having a meshing parameter $h$. The corresponding nonlocal models have condition numbers that depend, in general, on both $\delta$ and $h$. For example, Du and Zhou (2017) give sharp lower and upper bounds for the condition number of the stiffness matrix corresponding to a finite element discretization of a nonlocal diffusion operator based on a quasi-uniform regular triangulation. Typically, if a fractionaltype translation-invariant kernel such as those satisfying (5.11) is used to describe nonlocal interactions, the condition number of the resulting discretized nonlocal diffusion model is of $O\left(h^{-2 s} \delta^{2 s-2}\right)$, where, as always, $\delta$ denotes the size of the horizon parameter. In practice, both $h$ and $\delta$ could be small parameters, so the view that nonlocal models yield better conditioned systems than their local counterparts should be taken with due care. Additional detailed estimates on the conditioning of nonlocal stiffness matrices can be found in Aksoylu and Unlu (2014).

For effective algebraic solvers of the resulting linear system, we refer to studies of the use of Toeplitz (Wang and Tian 2012, Vollmann and Schulz 2019) and multigrid solvers (Du and Zhou 2017).

\subsection{Fast algorithm for kernels with non-smooth truncation}

Tian and Engquist (2019) have developed methods based on fast multipole methods (FMMs) and hierarchical matrix techniques. Their key observation is that the non-smooth transition of the kernel function typically used in peridynamics and nonlocal diffusion models can reduce the effectiveness of many standard fast solvers that are based on the compression of far-field interactions. A typical kernel $\gamma$ used in practice, shown in Figure 12.1(a), has a singularity at origin and its nonlocal interaction is truncated at a finite distance. The kernel is then decomposed into two parts, $\gamma_{1}$ and $\gamma_{2}$, as illustrated in Figure 12.1(b,c). The first part $\left(\gamma_{1}\right)$ is smooth away from the origin, so fast solvers using FMMs or hierarchical matrix techniques for the compression of far-field interactions can be successfully applied. The second part $\left(\gamma_{2}\right)$ is smooth inside the support. Tian and Engquist (2019) developed 


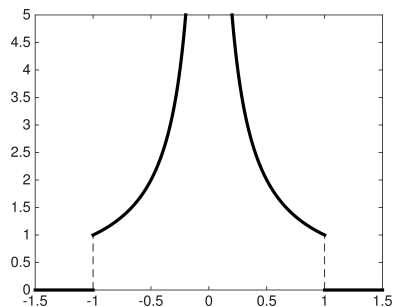

(a)

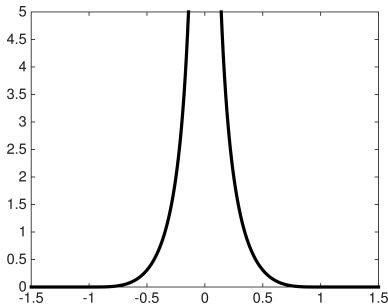

(b)

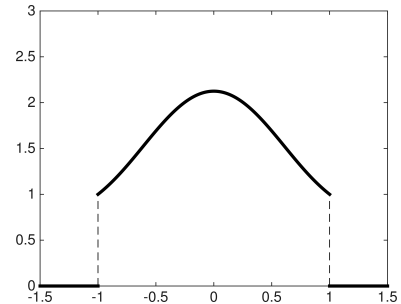

(c)

Figure 12.1. The kernel $\gamma(x)$ (a) splits into $\gamma_{1}(x)(\mathrm{b})$ and $\gamma_{2}(x)(\mathrm{c})$.

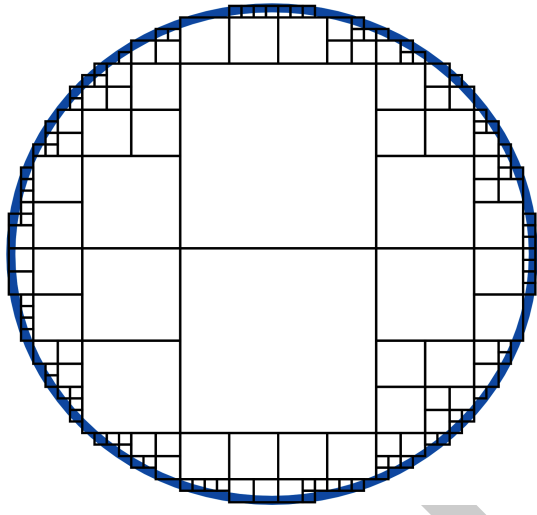

(a)

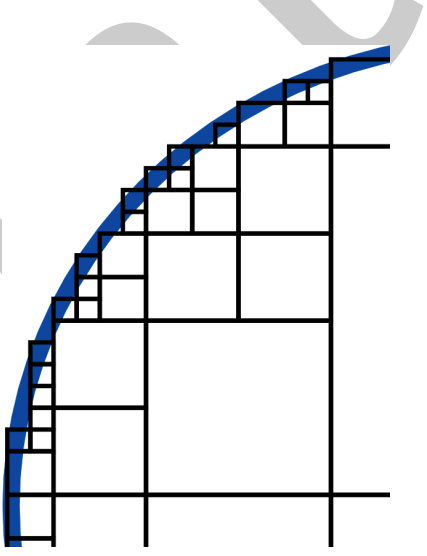

(b)

Figure 12.2. The circular region represents the interaction region corresponding to a point located at its centre. The decomposition of the circular region into hierarchical boxes is illustrated, with plot (b) being a close-up of plot (a).

an FMM-type algorithm for the fast evaluation of nonlocal operators with such a kernel function. The key idea is to compress the nonlocal interaction away from the boundary of the support. This idea is depicted in Figure 12.2, where the geometric boundary of the interaction kernel centred at a certain point is finely resolved by small boxes, whereas away from the boundary large boxes are used because there the kernel function is smooth. Because the number of small boxes needed to resolve co-dimension 1 surfaces increases with dimension, the complexity of the algorithm also increases with dimension. Tian and Engquist (2019) showed that the optimal complexity $O(N \log N)$ can be achieved for $N$ unknowns in one dimension. In higher dimensions there is algebraic complexity $O\left(N^{2-1 / d}\right)$, where $d$ is the spatial dimension of the problem. 


\subsection{Conditioning and solvers for finite element discretizations of the integral fractional Laplacian model}

Ainsworth, McLean and Tran (1999) gave the following results for the stiffness matrices resulting from finite element methods for the integral fractional Laplacian model.

For $s<d / 2$ and a family of shape-regular triangulations $\mathcal{T}_{h}$ with minimal and maximal element size $h_{\min }$ and $h$, the spectrum of the stiffness matrix $\boldsymbol{A}_{s}$ satisfies

$$
c N_{h}^{-2 s / d} h_{\min }^{d-2 s} \boldsymbol{I} \leq \boldsymbol{A}_{s} \leq C h^{d-2 s} \boldsymbol{I}
$$

and

$$
c N_{h}^{-2 s / d} \boldsymbol{I} \leq\left(\boldsymbol{D}^{s}\right)^{-1 / 2} \boldsymbol{A}_{s}\left(\boldsymbol{D}^{s}\right)^{-1 / 2} \leq C \boldsymbol{I},
$$

where $\boldsymbol{D}^{s}$ denotes the diagonal part of $\boldsymbol{A}_{s}$. Moreover, the condition number of the stiffness matrix satisfies

$$
\kappa\left(\boldsymbol{A}_{s}\right)=C\left(\frac{h}{h_{\min }}\right)^{d-2 s} N_{h}^{2 s / d} \text { and } \quad \kappa\left(\left(\boldsymbol{D}^{s}\right)^{-1} \boldsymbol{A}_{s}\right)=C N_{h}^{2 s / d} .
$$

If an implicit time-stepping scheme is used for the fractional-order heat equation (15.4), systems having matrices of the form

$$
\boldsymbol{M}+\Delta t \boldsymbol{A}_{s}
$$

need to be solved, where $\boldsymbol{M}$ denotes the usual finite element mass matrix. Ainsworth and Glusa (2017) have given the following result about the condition number of $\boldsymbol{M}+\Delta t \boldsymbol{A}_{s}$.

For a shape-regular and globally quasi-uniform family of triangulations $\mathcal{T}_{h}$ and for a time step $\Delta t \leq 1$,

$$
\kappa\left(\boldsymbol{M}+\Delta t \boldsymbol{A}_{s}\right) \leq C\left(1+\frac{\Delta t}{h^{2 s}}\right) .
$$

More generally, for a family of triangulations that is only locally quasiuniform and $\Delta t \leq h_{\min }^{2 s} N_{h}^{2 s / d}$, we have

$$
\kappa\left(\boldsymbol{M}+\Delta t \boldsymbol{A}_{s}\right) \leq C\left(\frac{h}{h_{\min }}\right)^{d}\left(1+\frac{\Delta t}{h^{2 s}}\right) .
$$

If $\boldsymbol{D}^{0}$ is taken to be the diagonal part of the mass matrix and $\Delta t \leq h^{2 s} N_{h}^{2 s / d}$, then

$$
\kappa\left(\left(\boldsymbol{D}^{0}\right)^{-1}\left(\boldsymbol{M}+\Delta t \boldsymbol{A}_{s}\right)\right) \leq C\left(1+\frac{\Delta t}{h_{\min }^{2 s}}\right) .
$$

These results show that for small fractional order $s$, if the time step $\Delta t$ is chosen small enough with respect to the mesh size, the conjugate gradient method with diagonal preconditioning will converge in a fixed number of 
steps. For larger fractional orders or for the steady-state problem, the number of iterations depends on the problem size. It has been shown that by applying a multigrid solver, one can restore a uniform bound on the number of iterations; see Sauter and Schwab (2010), Hackbusch $(1985,1994)$, Ainsworth and McLean (2003) and Ainsworth and Glusa (2017, 2018b).

We also observe that the system matrix $\boldsymbol{A}_{s}$ is entirely dense, owing to the nonlocal interactions. This means that for the efficient solution, efficient techniques for computing matrix-vector products with $\boldsymbol{A}_{s}$ need to be used. In the literature, fast transforms and matrix compression (Ainsworth and Glusa 2018b, Ainsworth and Glusa 2017) have been explored. The drawback of the former is their limitation to uniform meshes, whereas the latter are more difficult to implement. Both approaches lead to quasi-optimal complexity, i.e. $O\left(N_{h} \log N_{h}\right)$ operations to solve the system.

We note that a brief discussion about solvers for extended fractional Laplacian problems is given in Remark 7.1.

\section{PART THREE Selected extensions}

So far in the article we have mostly focused on steady-state nonlocal diffusion models, including fractional models. In this part we provide brief accounts of the extension of the models we have considered to other settings, along with additional approaches for obtaining approximate solutions of the models and a few applications of the models.

\section{Weakly coercive, indefinite and non-self-adjoint problems}

The discussion about discretization schemes given in Part 2 dealt with problems that fall into the category of what are known as Rayleigh-Ritz or strongly coercive problems. As such, the Lax-Milgram theorem is a fundamental tool in proving well-posedness of both continuous and discrete problems. In this section we consider more general settings involving indefinite and non-self-adjoint problems, settings for which the Lax-Milgram theorem cannot be applied.

\subsection{Indefinite and non-self-adjoint problems}

Instead of the symmetric and coercive bilinear form (2.10) (see also (20.2)), we now consider bilinear forms that have neither of these properties. As such, the bilinear forms considered in this section can be used in many settings that cannot be treated using the bilinear form (2.10). The discussion 
here largely follows the formulations given in Tian and Du (2020) for a more general class of parametrized problems.

The bilinear form $\mathcal{B}_{\delta}(\cdot, \cdot)$ is defined on a trial space $V_{\delta}$ and test space $W_{\delta}$ and satisfies the following requirements.

(i) $\mathcal{B}_{\delta}$ is bounded: there exists a constant $C_{b}>0$ independent of $\delta$ such that

$$
\mathcal{B}_{\delta}(u, v) \leq C_{b}\|u\|_{V_{\delta}}\|v\|_{W_{\delta}} \quad \text { for all } u \in V_{\delta}, v \in W_{\delta} .
$$

(ii) Inf-sup condition: there exists a constant $C_{c}>0$ independent of $\delta$ such that

$$
\inf _{u \in V_{\delta}} \sup _{v \in W_{\delta}} \frac{\mathcal{B}_{\delta}(u, v)}{\|u\|_{V_{\delta}}\|v\|_{W_{\delta}}} \geq C_{c}>0 .
$$

(iii) If $\mathcal{B}_{\delta}(u, v)=0$ for all $u \in V_{\delta}$, then $v=0$.

These conditions, as first shown by Necas $(1967,2016)$, guarantee that the problem

Find $u(\boldsymbol{x}) \in V_{\delta}$ such that $\mathcal{B}_{\delta}(u, v)=\langle f, v\rangle$ for all $v \in W_{\delta}$

is well-posed, provided that the linear functional on the right-hand side is bounded. Note that there are no symmetry or self-adjoint conditions placed on the bilinear form in (13.1). Note also that the discrete inf-sup condition is automatically satisfied in the case of coercive self-adjoint problems with $V_{\delta, h}=W_{\delta, h}$. Otherwise, it has to be verified for the chosen finite element spaces and the problem under consideration. Problems such as (13.1) that feature different test and trial spaces are often referred to as Petrov-Galerkin formulations. If $W_{\delta}=V_{\delta}$, then (13.1) is often referred to as a weakly coercive formulation.

Conforming discretizations are defined, as introduced in Tian and Du (2020) for the parametrized setting under consideration here, by choosing approximation subspaces $V_{\delta, h} \subset V_{\delta}$ and $W_{\delta, h} \subset W_{\delta}$ satisfying the following requirements.

(1) For a given $\delta \in\left(0, \delta_{0}\right)$, the family $\left\{W_{\delta, h}, h \in\left(0, h_{0}\right]\right\}$ of discrete subspaces of $W_{\delta}$ is dense in $W_{\delta}$ as $h \rightarrow 0$.

(2) Discrete inf-sup condition: there exists a constant $\widetilde{C}_{c}>0$, independent of $\delta$ and $h$, such that

$$
\inf _{u \in V_{\delta, h}} \sup _{v \in W_{\delta, h}} \frac{\mathcal{B}_{\delta}(u, v)}{\|u\|_{V_{\delta}}\|v\|_{W_{\delta}}} \geq \widetilde{C}_{c}>0 .
$$

These conditions, as first shown in Babuška (1971) and Babuška and Aziz (1972), guarantee that for a given $\delta \in\left(0, \delta_{0}\right)$ the problem

Find $u(\boldsymbol{x}) \in V_{\delta, h}$ such that $\mathcal{B}_{\delta}(u, v)=\langle f, v\rangle$ for all $v \in W_{\delta, h}$ 
is well-posed. Note that the independence of the constants $C_{b}, C_{c}$ and $\widetilde{C}_{c}$ on the parameter $\delta$ is not required if one is only interested in solving the problem for a fixed $\delta$, as in the original theory of Babuška (1971) and Babuška and Aziz (1972). It is imposed here for the study of asymptotic compatibility discussed next in Section 13.2 (Tian and Du 2020).

Problem (13.1) includes several important settings such as nonlocal mixed and operator-splitting formulations (see Section 13.3), nonlocal convectiondiffusion problems (see Section 14), nonlocal diffusion models and bondbased peridynamic models involving both attractive and repulsive interactions (Mengesha and Du 2013), and nonlocal systems such as the nonlocal Stokes equation introduced in Du and Tian (2019) and Lee and Du (2020).

\subsection{Asymptotically compatible schemes}

The discrete problem (13.2) involves the horizon parameter $\delta$ and the grid size parameter $h$ so that the asymptotic compatibility of particular choices of finite element spaces should be questioned. A study of this question is given in Tian and Du (2020) based on an extension of the original AC framework presented in Tian and Du (2014). The latter deals with only symmetric and coercive bilinear forms of the types similar to (2.10) and (20.2).

Concerning the discrete approximations, in addition to the requirements (1) and (2) listed in Section 13.1, we impose the following requirement (Tian and Du 2020).

(3) The family of discrete subspaces of $\left\{W_{\delta, h}\right\}$ is asymptotically dense in $W_{0}$ as $\delta \rightarrow 0$ and $h \rightarrow 0$, in the sense of definition (5.6). Here $W_{0}$ refers to the energy space for the original continuum local PDE problem corresponding to (13.1) and (13.2).

Now any scheme that satisfies requirements (1), (2) and (3) is provably an AC scheme (Tian and Du 2020).

\subsection{Operator-splitting and mixed formulations}

PDE equations such as $-\nabla \cdot(\boldsymbol{D} \nabla u)=f$ are often derived by first postulating a balance law $\nabla \cdot \boldsymbol{w}=f$ and choosing a constitutive (Darcy, Fick, Fourier, Ohm, etc.) law $\boldsymbol{w}=-\boldsymbol{D} \nabla u$. In some settings there are advantages to directly solving the two first-order equations instead of the single secondorder equation, perhaps the most important and useful being that wellposedness can be proved for $u \in L^{2}(\Omega)$ instead of $H^{1}(\Omega)$, as is the case for the second-order equation. On the other hand, there are problems that are most often posed as a mixed formulation, the most common being the Stokes equations for incompressible flows. 
One can mimic the local setting by recasting problem (1.5) with $\mathcal{L}_{\delta} u=$ $-\mathcal{D}_{\delta} \cdot\left(\mathcal{D}_{\delta}^{*} u\right)$ in $\Omega$ and with, say, $u=0$ on $\Omega_{\mathcal{I}_{\delta}}$, into the equivalent operatorsplitting formulation

$$
\begin{cases}\mathcal{D}_{\delta} \boldsymbol{\nu}(\boldsymbol{x}, \boldsymbol{y})=f(\boldsymbol{x}) & \text { for all } \boldsymbol{x} \in \Omega, \\ \boldsymbol{\nu}(\boldsymbol{x}, \boldsymbol{y})=\mathcal{D}_{\delta}^{*} u & \text { for all } \boldsymbol{x}, \boldsymbol{y} \in \Omega \cup \Omega_{\mathcal{I}_{\delta}},\end{cases}
$$

along with $u(\boldsymbol{x})=0$ for all $\boldsymbol{x} \in \Omega_{\mathcal{I}_{\delta}}$. A corresponding mixed weak formulation is given by

$$
\left\{\begin{array}{l}
(\boldsymbol{\nu}(\boldsymbol{x}, \boldsymbol{y}), \boldsymbol{\mu}(\boldsymbol{x}, \boldsymbol{y}))_{L^{2}\left(\left[\Omega \cup \Omega_{\mathcal{I}_{\delta}}\right]^{2}\right)}-\left(\mathcal{D}_{\delta} \boldsymbol{\mu}(\boldsymbol{x}, \boldsymbol{y}), u(\boldsymbol{x})\right)=0 \\
\left(\mathcal{D}_{\delta} \boldsymbol{\nu}(\boldsymbol{x}, \boldsymbol{y}), v(\boldsymbol{x})\right)=(f(\boldsymbol{x}), v(\boldsymbol{x}))
\end{array}\right.
$$

along with $u(\boldsymbol{x})=0$ for all $\boldsymbol{x} \in \Omega_{\mathcal{I}_{\delta}}$, where $(\cdot, \cdot)_{L^{2}\left(\left[\Omega \cup \Omega_{\mathcal{I}_{\delta}}\right]^{2}\right)}$ and $(\cdot, \cdot)$ denote $L^{2}$ inner products on the respective domains and the pairs $\boldsymbol{\nu}, \boldsymbol{\mu}$ and $u, v$ belong to appropriate function spaces.

Problem (13.4) can be treated as an extension of problem (13.1) with the bilinear form

$$
\begin{aligned}
\mathcal{B}_{\delta}((\boldsymbol{\nu}, u),(\boldsymbol{\mu}, v))= & (\boldsymbol{\nu}(\boldsymbol{x}, \boldsymbol{y}), \boldsymbol{\mu}(\boldsymbol{x}, \boldsymbol{y}))-\left(\mathcal{D}_{\delta} \boldsymbol{\mu}(\boldsymbol{x}, \boldsymbol{y}), u(\boldsymbol{x})\right) \\
& +\left(\mathcal{D}_{\delta} \boldsymbol{\nu}(\boldsymbol{x}, \boldsymbol{y}), v(\boldsymbol{x})\right) .
\end{aligned}
$$

While one may attempt to adopt the general inf-sup theory in Tian and $\mathrm{Du}$ (2020) to study the mixed weak formulation, the mixed finite element approximations would require the discretization of $\boldsymbol{\nu}(\boldsymbol{x}, \boldsymbol{y})$ and $\boldsymbol{\mu}(\boldsymbol{x}, \boldsymbol{y})$ in both $\boldsymbol{x}$ and $\boldsymbol{y}$. On the other hand, finite element discretizations of nonlocal models based on the operator-splitting formulation can be found in $\mathrm{Du}$, Ju and $\mathrm{Lu}(2019 a, 2019 b)$ for interaction kernels that are both radial and integrable, and that recover the local discontinuous Galerkin (LDG) discretization of the local PDE problem as $\delta \rightarrow 0$.

The nonlocal Stokes model is another example that can be formulated as a system in mixed form; see the discussions of Du and Tian (2019) and Lee and Du (2020), who also analysed spectral and finite difference approximations in a periodic boundary condition setting.

\section{Nonlocal convection-diffusion problems}

In this section we consider nonlocal analogues of the local convectiondiffusion (also referred to as advection-diffusion) problem

$$
\begin{cases}-\nabla \cdot(\boldsymbol{D} \nabla u)+\nabla \cdot(\boldsymbol{U} u)=f & \text { for all } \boldsymbol{x} \in \Omega, \\ u=0 & \text { for all } \boldsymbol{x} \in \partial \Omega\end{cases}
$$


where $\boldsymbol{U}(\boldsymbol{x})$ denotes a given velocity field and $\boldsymbol{D}(\boldsymbol{x})$ denotes a given symmetric, positive definite matrix. The most common approach towards defining a nonlocal convection-diffusion model is to replace the second-order diffusion term in (14.1) with a nonlocal analogue $-\mathcal{L} u$ but to keep the convection term as it is in (14.1). Such classical convection-nonlocal diffusion problems have been investigated for fractional models, including their connection to Lévy jump processes; see e.g. Meerschaert and Sikorskii (2012).

However, we consider fully nonlocal analogues of (14.1) in which the convection term in (14.1) is also replaced by a nonlocal analogue. As a result, the nonlocal convection-diffusion models we consider feature non-symmetric kernels $\gamma_{\delta}(\boldsymbol{x}, \boldsymbol{y})$, so they can also be viewed as modelling non-symmetric diffusion. Among other descriptions of non-symmetric diffusion that are not necessarily related to stochastic processes, we mention Baeumer and Meerschaert (2010), Meerschaert, Benson and Bäumer (1999) and Meerschaert and Sikorskii (2012) where the equations are set either in free space or in bounded domains, Ervin and Roop (2007) that treats the same problem in a variational setting, Felsinger, Kassmann and Voigt (2015) that analyses a variational formulation of non-symmetric diffusion for integrable kernels with square-integrable symmetric parts and for non-integrable kernels, and Andreu, Mazón, Rossi and Toledo (2010) that considers the strong form of non-symmetric diffusion equations for kernels that are positive, translationinvariant and integrable.

Based on D'Elia et al. (2017), we consider the most general form of a nonlocal analogue of (14.1), treating the two nonlocal terms as separate phenomena. Note that D'Elia et al. (2017) provide a generalization of Du, Huang and Lehoucq (2014) to a more general class of kernels.

\subsection{Non-symmetric kernels and nonlocal convection-diffusion operators}

Let $\mathcal{L}_{c d, \delta}$ denote the nonlocal convection-diffusion operator defined as

$$
-\mathcal{L}_{c d, \delta} u(\boldsymbol{x}):=\mathcal{D}_{d, \delta_{d}}\left(\boldsymbol{\Theta} \mathcal{D}_{d, \delta_{d}}^{*} u\right)(\boldsymbol{x})+\mathcal{D}_{c, \delta_{c}}(\boldsymbol{\mu} u)(\boldsymbol{x}),
$$

where $\mathcal{D}_{d, \delta_{d}}$ and $\mathcal{D}_{c, \delta_{c}}$ are nonlocal divergence operators associated with the anti-symmetric functions $\boldsymbol{\alpha}_{d}(\boldsymbol{x}, \boldsymbol{y})$ and $\boldsymbol{\alpha}_{c}(\boldsymbol{x}, \boldsymbol{y})$ and where $\boldsymbol{\mu}(\boldsymbol{x}, \boldsymbol{y})=$ $\boldsymbol{\mu}(\boldsymbol{y}, \boldsymbol{x})$ is a given function. Note that we allow for different horizons and different kernel functions for the diffusion and convection terms. We refer to the second-order tensor $\boldsymbol{\Theta}(\boldsymbol{x}, \boldsymbol{y})$ as the nonlocal diffusion tensor (see Section 1.1) and to the vector $\boldsymbol{\mu}(\boldsymbol{x}, \boldsymbol{y})$ as the nonlocal convection 'velocity'. Specifically, from (1.17), we have

$$
\begin{aligned}
& \mathcal{D}_{d, \delta_{d}}\left(\boldsymbol{\Theta} \mathcal{D}_{d, \delta_{d}}^{*} u\right)(\boldsymbol{x}) \\
& \quad=-2 \int_{\mathbb{R}^{d}}(u(\boldsymbol{y})-u(\boldsymbol{x})) \boldsymbol{\alpha}_{d}(\boldsymbol{x}, \boldsymbol{y}) \cdot\left(\boldsymbol{\Theta}(\boldsymbol{x}, \boldsymbol{y}) \boldsymbol{\alpha}_{d}(\boldsymbol{x}, \boldsymbol{y})\right) \mathcal{X}_{B_{\delta_{d}}(\boldsymbol{x})}(\boldsymbol{y}) \mathrm{d} \boldsymbol{y}
\end{aligned}
$$


and similarly, from (1.13) with $\boldsymbol{\nu}(\boldsymbol{x}, \boldsymbol{y})=\boldsymbol{\mu}(\boldsymbol{x}, \boldsymbol{y}) u(\boldsymbol{x})$, we have

$$
\begin{aligned}
\mathcal{D}_{c, \delta_{c}}(\boldsymbol{\mu} u)(\boldsymbol{x}) & =\int_{\mathbb{R}^{d}}(\boldsymbol{\mu}(\boldsymbol{x}, \boldsymbol{y}) u(\boldsymbol{x})+\boldsymbol{\mu}(\boldsymbol{y}, \boldsymbol{x}) u(\boldsymbol{y})) \cdot \boldsymbol{\alpha}_{c}(\boldsymbol{x}, \boldsymbol{y}) \mathcal{X}_{B_{\delta_{c}}(\boldsymbol{x})}(\boldsymbol{y}) \mathrm{d} \boldsymbol{y} \\
& =\int_{\mathbb{R}^{d}}(u(\boldsymbol{x})+u(\boldsymbol{y})) \boldsymbol{\mu}(\boldsymbol{x}, \boldsymbol{y}) \cdot \boldsymbol{\alpha}_{c}(\boldsymbol{x}, \boldsymbol{y}) \mathcal{X}_{B_{\delta_{c}}(\boldsymbol{x})}(\boldsymbol{y}) \mathrm{d} \boldsymbol{y} .
\end{aligned}
$$

Setting

$$
\begin{aligned}
\gamma_{c d, \delta}(\boldsymbol{x}, \boldsymbol{y}):= & \underbrace{2 \boldsymbol{\alpha}_{d}(\boldsymbol{x}, \boldsymbol{y}) \cdot\left(\boldsymbol{\Theta}(\boldsymbol{x}, \boldsymbol{y}) \boldsymbol{\alpha}_{d}(\boldsymbol{x}, \boldsymbol{y})\right) \mathcal{X}_{B_{\delta_{d}}(\boldsymbol{x})}(\boldsymbol{y})}_{\text {symmetric part } \gamma_{d, \delta_{d}}(\boldsymbol{x}, \boldsymbol{y})} \\
& \underbrace{-\boldsymbol{\mu}(\boldsymbol{x}, \boldsymbol{y}) \cdot \boldsymbol{\alpha}_{c}(\boldsymbol{x}, \boldsymbol{y}) \mathcal{X}_{B_{\delta_{c}}(\boldsymbol{x})}(\boldsymbol{y})}_{\text {anti-symmetric part } \gamma_{c, \delta_{c}}(\boldsymbol{x}, \boldsymbol{y})},
\end{aligned}
$$

we can rewrite $(14.2)$ as

$$
\mathcal{L}_{c d, \delta} u(\boldsymbol{x})=\int_{\mathbb{R}^{d}}\left(u(\boldsymbol{y}) \gamma_{c d, \delta}(\boldsymbol{y}, \boldsymbol{x})-u(\boldsymbol{x}) \gamma_{c d, \delta}(\boldsymbol{x}, \boldsymbol{y})\right) \mathrm{d} \boldsymbol{y} \quad \text { for all } \boldsymbol{x} \in \mathbb{R}^{d}
$$

In the case of $\boldsymbol{\alpha}_{c}(\boldsymbol{x}, \boldsymbol{y})=\boldsymbol{\alpha}_{d}(\boldsymbol{x}, \boldsymbol{y})$ and $\delta_{d}=\delta_{c}$, this non-symmetric diffusion operator and the corresponding initial value problem are analysed for a special class of kernels in Du et al. (2014).

Note that with $\delta=\max \left\{\delta_{d}, \delta_{c}\right\}$, we have

$$
\gamma_{c d, \delta}(\boldsymbol{x}, \boldsymbol{y})=0 \text { for all } \boldsymbol{y} \notin B_{\delta}(\boldsymbol{x}) .
$$

Also note that although $\boldsymbol{\alpha}_{d}(\boldsymbol{x}, \boldsymbol{y})$ and $\boldsymbol{\alpha}_{c}(\boldsymbol{x}, \boldsymbol{y})$ are often radial functions, in general $\boldsymbol{\Theta}(\boldsymbol{x}, \boldsymbol{y})$ and $\boldsymbol{\mu}(\boldsymbol{x}, \boldsymbol{y})$ are not, so that, also in general, $\gamma_{c d, \delta}(\boldsymbol{x}, \boldsymbol{y})$ is not radial or translationally invariant. See Remark 2.3.

Tian, Ju and Du (2015, 2017) and Lee and Du (2019) considered AC discretizations of nonlocal convection-diffusion problems. Du et al. (2014) considered a convection-diffusion operator in one dimension, which turns out to be a special case of the general nonlocal convection-diffusion operator (14.2) if we choose $\delta_{d}=\delta_{c}, \Theta(x, y)=\kappa=$ constant, $\left(\alpha_{d}(x, y)\right)^{2}=\sigma_{d, \delta}(\mid y-$ $x \mid), \mu(x, y)=U$ a constant, and $\alpha_{c}(x, y)=(y-x) \sigma_{c, \delta}(|y-x|)$, with $\sigma_{d, \delta}(\cdot)$ and $\sigma_{c, \delta}(\cdot)$ being even functions having unit second moments. The resulting operator is given by

$$
\begin{aligned}
\mathcal{L}_{c d, \delta} u(x)= & 2 \kappa \int_{\mathbb{R}}(u(y)-u(x)) \sigma_{d, \delta}(|y-x|) \mathrm{d} y \\
& -U \int_{R}(u(y)+u(x))(y-x) \sigma_{c, \delta}(|y-x|) \mathrm{d} y .
\end{aligned}
$$

The local counterpart of this operator is $\mathcal{L}_{0} u(x)=\kappa u^{\prime \prime}(x)+U u^{\prime}(x)$. One may also connect this nonlocal convection diffusion model with non-symmetric jump processes; see Du et al. (2014). 
Other nonlocal convection-diffusion models are presented in Tian, Ju and Du (2017), including some that are reminiscent of state-based peridynamic models. For example, a conservative formulation is defined by the nonlocal convection-diffusion operator

$$
\begin{aligned}
& \mathcal{L}_{c d, \delta} u(\boldsymbol{x}) \\
& =\int_{\Omega \cup \Omega_{\mathcal{I}_{\delta}}}(A(\boldsymbol{x})+A(\boldsymbol{y}))(u(\boldsymbol{y})-u(\boldsymbol{x})) \sigma_{d, \delta}(|\boldsymbol{y}-\boldsymbol{x}|)(|\boldsymbol{y}-\boldsymbol{x}|) \mathcal{X}_{c}(\boldsymbol{x}, \boldsymbol{y}) \mathrm{d} \boldsymbol{y} \\
& +\int_{\Omega \cup \Omega_{\mathcal{I}_{\delta}}}\left(\boldsymbol{b}(\boldsymbol{x}) u(\boldsymbol{x}) \mathcal{X}_{c}(\boldsymbol{x}, \boldsymbol{y})+\boldsymbol{b}(\boldsymbol{y}) u(\boldsymbol{y}) \mathcal{X}_{c}(\boldsymbol{y}, \boldsymbol{x})\right) \cdot(\boldsymbol{y}-\boldsymbol{x}) \sigma_{c, \delta}(|\boldsymbol{y}-\boldsymbol{x}|) \mathrm{d} \boldsymbol{y},
\end{aligned}
$$

where we have the indicator function

$$
\mathcal{X}_{c}(\boldsymbol{x}, \boldsymbol{y})= \begin{cases}1 & \text { if }|\boldsymbol{y}-\boldsymbol{x}|<\delta \text { and } \boldsymbol{b}(\boldsymbol{x}) \cdot(\boldsymbol{y}-\boldsymbol{x})>0 \\ 0 & \text { otherwise }\end{cases}
$$

which is generically non-symmetric and is dependent on the velocity field $\boldsymbol{b}(\boldsymbol{x})$. This operator can also be shown to be a special case of operator given in (14.2). Its local counterpart is

$$
\mathcal{L}_{0} u(\boldsymbol{x})=\nabla \cdot(A(\boldsymbol{x}) \nabla u(\boldsymbol{x}))+\nabla \cdot(\boldsymbol{b}(\boldsymbol{x}) u(\boldsymbol{x})) .
$$

Although AC discretizations of the models using these operators have been discussed by Tian, Ju and Du $(2015,2017)$ and Lee and Du (2019), the attendant analyses are done using different techniques and for specialized kernels. One may apply the general framework given in Tian and Du (2020) to possibly offer a unified treatment of systems of non-self-adjoint problems. Indeed, additional studies of asymptotically compatible schemes may also shed new light on improving the robustness of numerical methods based on various nonlocal smoothing approaches, that are in use for local PDE models such as smoothed particle hydrodynamics, as well as the construction of well-posed nonlocal models such as the nonlocal Stokes equation considered in Du and Tian (2019) and Lee and Du (2019).

\subsubsection{Steady-state nonlocal convection-diffusion problems}

The nonlocal analogue of (14.1) is given by

$$
\begin{cases}-\mathcal{L}_{c d, \delta} u=f & \boldsymbol{x} \in \Omega, \\ u=0 & \boldsymbol{x} \in \Omega_{\mathcal{I}_{\delta}} .\end{cases}
$$

Weak formulations corresponding to (14.6) can be defined in the usual way. Simplifying some notation, for $\ell=d$ or $\ell=c$, one can define the constrained energy space

$$
V_{\ell}^{0}:=\left\{v \in L^{2}\left(\Omega \cup \Omega_{\mathcal{I}_{\delta}}\right):|v|_{V_{\ell}^{0}}<\infty \text { and } v=0 \text { on } \Omega_{\mathcal{I}_{\delta}}\right\}
$$


for which the semi-norm

$$
|v|_{V_{\ell}^{0}}:=\frac{1}{2} \int_{\Omega \cup \Omega_{\mathcal{I}_{\delta}}} \int_{\Omega \cup \Omega_{\mathcal{I}_{\delta}}}\left|\mathcal{D}_{\ell}^{*}(v)(\boldsymbol{x}, \boldsymbol{y})\right|^{2} \gamma_{d, \delta_{\ell}}(\boldsymbol{x}, \boldsymbol{y}) \mathrm{d} \boldsymbol{y} \mathrm{d} \boldsymbol{x}
$$

is a norm. We assume that the norm $|v|_{V_{\ell}^{0}}$ satisfies the nonlocal Poincaré inequality $\|v\|_{L^{2}\left(\Omega \cup \Omega_{\mathcal{I}_{\delta}}\right)} \leq C_{p}|v|_{V_{\ell}^{0}}$ for all $v \in V_{\ell}^{0}$ and that $V_{d}^{0} \subset V_{c}^{0}$ so that $|v|_{V_{c}^{0}} \leq|v|_{V_{d}^{0}}$. The latter assumption implies that solution operators for nonlocal diffusion problems under consideration effect greater smoothing compared to those for nonlocal convection problems, as is the case for local partial differential operators.

Let the bilinear form $\mathcal{A}_{c d, \delta}(\cdot, \cdot)$ be defined, for all $u, v \in V_{d}^{0}$, by

$$
\begin{aligned}
\mathcal{A}_{c d, \delta}(u, v)= & \int_{\Omega \cup \Omega_{\mathcal{I}_{\delta}}} \int_{\Omega \cup \Omega_{\mathcal{I}_{\delta}}} \mathcal{D}_{d, \delta}^{*}(u)(\boldsymbol{x}, \boldsymbol{y}) \cdot\left(\boldsymbol{\Theta} \mathcal{D}_{d, \delta}^{*} v\right)(\boldsymbol{x}, \boldsymbol{y}) \mathrm{d} \boldsymbol{y} \mathrm{d} \boldsymbol{x} \\
& -\int_{\Omega} \mathcal{D}_{c, \delta}(\boldsymbol{\mu} u)(\boldsymbol{x}) v(\boldsymbol{x}) \mathrm{d} \boldsymbol{x}
\end{aligned}
$$

and let the linear functional $\langle f, v\rangle$ be defined, for all $v \in V_{d}^{0}$, by

$$
\langle f, v\rangle=\int_{\Omega} f(\boldsymbol{x}) v(\boldsymbol{x}) \mathrm{d} \boldsymbol{x} .
$$

Then a weak formulation of (14.6) is given as follows: given $f$ belonging to the dual space of $V_{d}^{0}$, find $u \in V_{d}^{0}$ that satisfies

$$
\mathcal{A}_{c d, \delta}(u, v)=\langle f, v\rangle \text { for all } v \in V_{d}^{0} .
$$

D'Elia et al. (2017) proved the well-posedness of (14.9) using three different approaches. Here we state results that mimic what is obtained for local convection-diffusion problems. Specifically,

- if $f(\boldsymbol{x})$ belongs to the dual space of $V_{d}^{0}$,

- if $\boldsymbol{\Theta}(\boldsymbol{x}, \boldsymbol{y})$ is such that there exist $\underline{\vartheta}, \bar{\vartheta}>0$ satisfying

$$
0<\underline{\vartheta} \leq \inf _{\boldsymbol{x} \in \mathbb{R}^{d}}\left(\min _{i} \theta_{i}\right) \quad \text { and } \sup _{\boldsymbol{x} \in \mathbb{R}^{d}}\left(\max _{i} \theta_{i}\right) \leq \bar{\vartheta}<\infty
$$

where $\theta_{i}$ denote the singular values of $\boldsymbol{\Theta}$,

- if $\boldsymbol{\mu}$ is such that $C_{p}^{2}\left\|\mathcal{D}_{c} \boldsymbol{\mu}\right\|_{\infty} \leq 2 \underline{\vartheta}$ and $\||\boldsymbol{\mu}|\|_{\infty} \leq \bar{\mu}$,

then problem (14.9) has a unique solution $u \in V_{d}^{0}$. Furthermore, that solution satisfies the a priori estimate

$$
|u|_{V_{d}^{0}} \leq C\|f\|_{V_{d}^{\prime}},
$$


where $C=1 / C_{\text {coer }}$ and

$$
C_{\text {coer }}=\underline{\vartheta}-\frac{1}{2} C_{p}^{2}\left\|\mathcal{D}_{c} \boldsymbol{\mu}\right\|_{\infty}
$$

denotes the coercivity constant for the bilinear form $\mathcal{A}_{c d, \delta}(\cdot, \cdot)$. Note that the above analysis is effective for diffusion-dominated problems. For the convection-dominated case one may use the formulation in Tian and Du (2020), discussed in Section 13.1, to get results in more general cases.

\section{Time-dependent nonlocal problems}

Although we do not consider nonlocal time-dependent problems other than in this subsection and briefly in some other sections, a brief discussion is warranted. We do not delve into nonlocality in time, for which there is a vast literature devoted to fractional time derivatives and other settings in which memory effects are present.

Weak formulations of time-dependent problems can be defined in the same manner as that for local PDE time-dependent problems, once one knows how to treat steady-state problems. Likewise, for the discretization of nonlocal time-dependent problems, spatial discretization can be effected using any discretization method for the corresponding steady-state problem, including those discussed in Part 2, and temporal discretization can be effected using any discretization method for the corresponding local PDE problem, e.g. the backward-Euler or Crank-Nicolson method for (15.1) or, for (15.7), a leap-frog or other explicit method. Furthermore, the analysis of weak formulations and discretizations of nonlocal time-dependent problems, including the derivation of well-posedness results and error estimates, also follows the same paths as those for the corresponding local PDE problems.

Here we only consider a small sample of the differences between timedependent local and nonlocal problems.

\subsection{Time-dependent nonlocal diffusion}

Using the notation used in (1.5), we have the nonlocal time-dependent diffusion equation

$$
\begin{cases}\rho \frac{\partial u}{\partial t}=\mathcal{L}_{\delta} u+f(\boldsymbol{x}, t) & \text { for all } \boldsymbol{x} \in \Omega \times(0, T], \\ \mathcal{V} u=g(\boldsymbol{x}, t) & \text { for all } \boldsymbol{x} \in \Omega_{\mathcal{I}_{\delta}} \times(0, T], \\ u(\boldsymbol{x}, 0)=u_{0}(\boldsymbol{x}) & \text { for all } \boldsymbol{x} \in \Omega,\end{cases}
$$

where $\rho(\boldsymbol{x})>0$ and $u_{0}(\boldsymbol{x})$ are given functions defined on $\Omega$ and $f(\boldsymbol{x}, t)$ and $g(\boldsymbol{x}, t)$ are given functions defined on $\Omega \times(0, T]$ and $\Omega_{\mathcal{I}_{\delta}} \times[0, T]$, respectively. 
Using the nonlocal Green's first identity, it is an easy matter to show, for $f=0$ and $g=0$, that the nonlocal diffusion equation (15.1) implies that

$$
\frac{1}{2} \frac{\mathrm{d}}{\mathrm{d} t} \int_{\Omega} u^{2} \mathrm{~d} \boldsymbol{x}+\int_{\Omega \cup \Omega_{\mathcal{I}_{\delta}}} \int_{\Omega \cup \Omega_{\mathcal{I}_{\delta}}} \mathcal{D}_{\delta}^{*} u \cdot\left(\boldsymbol{\Theta}_{\delta} \mathcal{D}_{\delta}^{*} u\right) \mathrm{d} \boldsymbol{y} \mathrm{d} \boldsymbol{x}=0,
$$

so that

$$
\int_{\Omega} u^{2}(\boldsymbol{x}, t) \mathrm{d} \boldsymbol{x} \leq \int_{\Omega} u_{0}^{2}(\boldsymbol{x}) \mathrm{d} \boldsymbol{x} \text { for all } t>0,
$$

and for time-independent $\boldsymbol{\Theta}_{\delta}$

$$
\begin{aligned}
& \int_{\Omega \cup \Omega_{\mathcal{I}_{\delta}}} \int_{\Omega \cup \Omega_{\mathcal{I}_{\delta}}} \mathcal{D}_{\delta}^{*} u(\boldsymbol{x}, t) \cdot\left(\boldsymbol{\Theta}_{\delta} \mathcal{D}_{\delta}^{*} u(\boldsymbol{x}, t) \mathrm{d} \boldsymbol{y} \mathrm{d} \boldsymbol{x}\right. \\
& \quad \leq \int_{\Omega \cup \Omega_{\mathcal{I}_{\delta}}} \int_{\Omega \cup \Omega_{\mathcal{I}_{\delta}}} \mathcal{D}_{\delta}^{*} u(\boldsymbol{x}, 0) \cdot\left(\boldsymbol{\Theta}_{\delta} \mathcal{D}_{\delta}^{*} u(\boldsymbol{x}, 0) \mathrm{d} \boldsymbol{y} \mathrm{d} \boldsymbol{x} \text { for all } t>0 .\right.
\end{aligned}
$$

These are decay characteristics of diffusive processes, for example, (15.2) and the local version of (15.3) hold for parabolic PDEs. However, for kernel functions that are both radial and integrable, although solutions of the nonlocal diffusion equation (15.1) satisfy the decay properties (15.2) and (15.3), unlike the case for parabolic PDEs, those solutions may not be much smoother than the data. One can also consider various types of nonlocalin-time versions of nonlocal diffusion equations; see for example Chen et al. (2017).

We note that the fractional heat equation

$$
\begin{cases}u_{t}+(-\Delta)^{s} u=f & \text { for all } \boldsymbol{x} \in \Omega, t \in(0, T), \\ u=g & \text { for all } \boldsymbol{x} \in \mathbb{R}^{d} \backslash \Omega, t \in(0, T), \\ u(\cdot, 0)=u_{0}(\boldsymbol{x}) & \text { for all } \boldsymbol{x} \in \Omega .\end{cases}
$$

is perhaps of even greater interest compared to the steady-state case. Similarly, one can consider the fractional heat equation with the regional fractional Laplacian; see Gal and Warma (2016).

\subsection{Time-dependent convection-diffusion problems}

For $T>0$, we introduce the time-dependent function spaces

$$
\begin{aligned}
L^{2}\left(0, T ; V_{d}^{0}\right) & =\left\{v(\cdot, t) \in V_{d}^{0}:|v(\cdot, t)|_{V_{d}} \in L^{2}(0, T)\right\}, \\
L^{2}\left(0, T ; V_{d}^{\prime}\right) & =\left\{f(\cdot, t) \in V_{d}^{\prime}:\|f(\cdot, t)\|_{V_{d}^{\prime}} \in L^{2}(0, T)\right\} .
\end{aligned}
$$

The time-dependent nonlocal convection-diffusion problem is then given by

$$
\begin{cases}u_{t}-\mathcal{L}_{c d, \delta} u=f & \text { for all } \boldsymbol{x} \in \Omega, t \in(0, T], \\ u(\boldsymbol{x}, t)=0 & \text { for all } \boldsymbol{x} \in \Omega_{\mathcal{I}_{\delta}}, t \in(0, T], \\ u(\boldsymbol{x}, 0)=u_{0}(\boldsymbol{x}) & \text { for all } \boldsymbol{x} \in \Omega\end{cases}
$$


where the operator $\mathcal{L}_{c d, \delta}$ is defined in (14.2). The corresponding weak formulation is as follows: given $f \in L^{2}\left(0, T ; V_{d}^{\prime}\right)$ and $u_{0} \in V_{d}^{0}$, find $u \in L^{2}\left(0, T ; V_{d}^{0}\right)$ that satisfies $u(\boldsymbol{x}, 0)=u_{0}(\boldsymbol{x})$ and, for all $v \in V_{d}^{0}$ and for almost every $t \in(0, T]$,

$$
\left(u_{t}, v\right)_{\Omega}+\mathcal{A}_{c d, \delta}(u, v)=\langle f, v\rangle,
$$

where $(\cdot, \cdot)_{\Omega}$ denotes the $L^{2}$ inner product over $\Omega$. The coercivity and continuity of $\mathcal{A}_{c d, \delta}(\cdot, \cdot)$ and the continuity of $\langle\cdot, \cdot\rangle$ guarantee that the weak formulation (15.6) is well-posed. However, as pointed out by D'Elia et al. (2017), standard arguments of variational theory (Evans 1998) imply that actually much weaker assumptions on $\boldsymbol{\mu}$ are required for well-posedness, namely $\left\|\mathcal{D}_{c, \delta} \boldsymbol{\mu}\right\|_{\infty}<\infty$, where $\boldsymbol{\mu}(\boldsymbol{x}, \boldsymbol{y})$ denotes the nonlocal convection 'velocity' introduced in (14.3).

\subsection{Nonlocal wave equations}

One can also consider the nonlocal wave equation

$$
\begin{cases}\rho \frac{\partial^{2} u}{\partial t^{2}}=\mathcal{L}_{\delta} u+f(\boldsymbol{x}, t) & \text { for all } \boldsymbol{x} \in \Omega \times(0, T] \\ \mathcal{V} u=g(\boldsymbol{x}, t) & \text { for all } \boldsymbol{x} \in \Omega_{\mathcal{I}_{\delta}} \times(0, T], \\ u(\boldsymbol{x}, 0)=u_{0}(\boldsymbol{x}) & \text { for all } \boldsymbol{x} \in \Omega, \\ \frac{\partial u}{\partial t}(\boldsymbol{x}, 0)=u_{1}(\boldsymbol{x}) & \text { for all } \boldsymbol{x} \in \Omega,\end{cases}
$$

where $\rho(\boldsymbol{x})>0, u_{0}(\boldsymbol{x}), f(\boldsymbol{x}, t)$ and $g(\boldsymbol{x}, t)$ are defined as for (15.1) and $u_{1}(\boldsymbol{x})$ is a given function defined on $\Omega$.

For (15.7) with $f=0$ and $g=0$, we have conservation of energy, that is,

$$
\frac{\mathrm{d}}{\mathrm{d} t}\left(\frac{1}{2} \int_{\Omega}\left(\frac{\mathrm{d} u}{\mathrm{~d} t}\right)^{2} \mathrm{~d} \boldsymbol{x}+\int_{\Omega \cup \Omega_{\mathcal{I}_{\delta}}} \int_{\Omega \cup \Omega_{\mathcal{I}_{\delta}}} \mathcal{D}_{\delta}^{*} u \cdot\left(\boldsymbol{\Theta}_{\delta} \mathcal{D}_{\delta}^{*} u\right) \mathrm{d} \boldsymbol{y} \mathrm{d} \boldsymbol{x}\right)=0,
$$

which is a characteristic of wave processes such as the PDE wave equation. One can find studies related to these nonlocal wave equations in Guan and Gunzburger (2015), Du et al. (2018d) and Du, Lipton and Mengesha $(2016 a)$.

One of the stark differences between local and nonlocal models is in their dispersion relations for wave equations. For the one-dimensional local PDE wave equation

$$
\frac{\partial^{2} u}{\partial^{2} t}=c^{2} \frac{\partial^{2} u}{\partial^{2} x}
$$


where $c$ is a constant, by setting

$$
u(x, t)=\mathrm{e}^{-\mathrm{i} \omega t+\mathrm{i} k x},
$$

we obtain the dispersion relation

$$
\omega^{2}=c^{2} k^{2}
$$

In fact, this relation shows that there is no dispersion. The velocity of the wave is $\omega / k= \pm c$, which is independent of $\omega$ and $k$.

Guan and Gunzburger (2015) showed that the one-dimensional nonlocal wave equation

$$
\frac{\partial^{2} u}{\partial^{2} t}=\frac{2-2 s}{\delta^{2-2 s}} c^{2} \int_{x-\delta}^{x+\delta} \frac{u(y, t)-u(x, t)}{|y-x|^{1+2 s}} \mathrm{~d} y, \quad 0 \leq s<1 / 2
$$

has the dispersion relation

$$
\omega^{2}=\frac{2-2 s}{\delta^{2-2 s}} c^{2} \int_{0}^{\delta} \frac{2-2 \cos (k y)}{y^{1+2 s}} \mathrm{~d} y .
$$

Observe that the wave velocity $\omega / k$ is a nonlinear function of $k$. Guan and Gunzburger (2015) also showed that as $\delta \rightarrow 0, \omega$ given by (15.9) converges (quadratically with respect to $\delta$ ) to the local $\omega$ of (15.8). Similar results are obtained there for the two-dimensional case. Similar dispersion relations have been discussed for nonlocal operators; see e.g. Zhou and Du (2010), Du and Zhou (2011) and Du (2019).

\section{Inverse problems}

Among the many challenges faced when dealing with nonlocal problems, we find, even more than for local PDE problems, that mathematical models are not known with exactitude; for example, source terms, volume constraint data, coefficients, and even the functional form of the kernel itself may be unknown or subject to uncertainty. If there are experimental data or other a priori information (that may be sparse and/or noisy) available about the state of the system or about an output of interest that depends on the state, one can then resort to control or optimization strategies to identify the unknown entities and thus define a data-driven mathematical model that is more faithful to the physics being considered.

Here we consider inverse problems for the nonlocal diffusion problem (1.5) in which the boundary operator $\mathcal{V}$ could correspond to Dirichlet, Neumann or Robin volume constraints. Let $V\left(\Omega \cup \Omega_{\mathcal{I}}\right)$ denote a function space for the state $u(\boldsymbol{x})$ and let $W$ denote a set of controls that could consist of function spaces or parameter vectors or a combination of both. Then a 
general inverse problem for nonlocal diffusion is given as follows:

Seek $u(\boldsymbol{x}) \in V\left(\Omega \cup \Omega_{\mathcal{I}}\right)$ and $\mu \in W$ such that

$$
\min _{\mu \in W} \mathcal{J}(u ; \mu)
$$

subject to (1.5) being satisfied, where

$$
\mathcal{J}(u ; \mu)=\mathcal{Q}(u ; \mu)+\mathcal{R}(\mu) .
$$

In (16.1), the first term $\mathcal{Q}(\cdot ; \cdot)$ in the objective functional $\mathcal{J}(\cdot ; \cdot)$ denotes a cost functional that depends on the state and control whereas $\mathcal{R}(\cdot)$ denotes a regularization functional that serves to guarantee the well-posedness of the problem. The control set $W$ could contain data functions such as $f$, $g$ and $\boldsymbol{\Theta}$, and also parameters appearing in the model definition such as the horizon $\delta$ or the fractional exponent $s$ if (1.5) represents a fractional Laplacian problem. The functions may be parametrized, in which case $W$ only contains a parameter vector. Additionally, the control set may contain constraints on the control; constraints on the state may be also be imposed. See Section 17 for an example of the latter. In some such cases, the regularization term in (16.1) may not be needed because such constraints may be sufficient to guarantee well-posedness.

The literature about the control and optimization of nonlocal problems is still limited; however, interest in such topics in the setting of nonlocal diffusion is quickly growing. Recent studies in this direction focus on the wellposedness and stability of the minimization problem (16.1), the asymptotic behaviour of its solution, and its numerical discretization. In particular, with respect to the latter, numerical convergence analyses, error estimates, and solver performance are of interest. In this section we provide brief reviews of selected contributions devoted to the control and optimization of nonlocal problems, including integral fractional models, treating both control and identification problems.

\subsection{Inverse problems for nonlocal diffusion}

In this section we focus on operators of the form of (1.6) with $\gamma_{\delta}(\boldsymbol{x}, \boldsymbol{y})$ given in terms of the kernel function $\phi_{\delta}(\boldsymbol{x}, \boldsymbol{y})$ and constitutive function $\theta_{\delta}(\boldsymbol{x}, \boldsymbol{y})$ by $(2.16),(2.17)$ and $(2.18)$, that is, we have

$$
\begin{aligned}
\mathcal{L}_{\delta} u(\boldsymbol{x}) & =\mathcal{D}_{\delta}\left(\boldsymbol{\Theta}_{\delta} \mathcal{D}^{*} u\right)(\boldsymbol{x}) \\
& =-2 \int_{\Omega \cup \Omega_{\mathcal{I}_{\delta}}}(u(\boldsymbol{y})-u(\boldsymbol{x})) \gamma_{\delta}(\boldsymbol{x}, \boldsymbol{y}) \mathrm{d} \boldsymbol{y} \\
& =-2 \int_{B_{\delta}(\boldsymbol{x})} \theta_{\delta}(\boldsymbol{x}, \boldsymbol{y})(u(\boldsymbol{y})-u(\boldsymbol{x})) \phi_{\delta}(\boldsymbol{x}, \boldsymbol{y}) \mathrm{d} \boldsymbol{y} \quad \text { for all } \boldsymbol{x} \in \Omega .
\end{aligned}
$$




\subsubsection{Distributed optimal control in nonlocal diffusion for a matching functional}

We consider the minimization problem (16.1) with the state space $V(\Omega \cup$ $\left.\Omega_{\mathcal{I}}\right)=V\left(\Omega \cup \Omega_{\mathcal{I}_{\delta}}\right)$, the operator $\mathcal{L}$ now given by (16.2), and with perhaps the most commonly used cost functional and regularization term for both PDE and nonlocal optimal control problems, namely

$$
\mathcal{J}(u ; \mu)=\frac{1}{2}\|u-\widehat{u}\|_{U(\Omega)}^{2}+\frac{\beta}{2}\|\mathcal{M}(\mu)\|_{W}^{2},
$$

where the first term is usually referred to as a matching functional, the second term as Tikhonov regularization, and the given function $\widehat{u}(\boldsymbol{x})$ for $\boldsymbol{x} \in \Omega$ as the target function. The operator $\mathcal{M}$ could be, for example, a local derivative or a nonlocal operator such as $\mathcal{D}_{\delta}^{*}$, that is chosen with the purpose of keeping $\mathcal{M}(\mu)$ under control and to either guarantee wellposedness or improve the conditioning of the problem. Furthermore, $\widehat{u}$ need not belong to state space $V\left(\Omega \cup \Omega_{\mathcal{I}_{\delta}}\right)$ and, in (16.3), we have norms $\|\cdot\|_{U(\Omega)}$ and $\|\cdot\|_{W}$ that are well-defined for $\widehat{u} \in U(\Omega)$ and $\mathcal{M}(\mu)$ with $\mu \in W$, respectively. Often target functions are not regular, so a reasonable choice is $U(\Omega)=L^{2}(\Omega)$.

Perhaps D'Elia and Gunzburger (2014) were the first to analyse this problem for square-integrable and also non-integrable kernel functions $\phi_{\delta}(\boldsymbol{x}, \boldsymbol{y})$, albeit for $\theta_{\delta}=1$ and $\mathcal{V} u=u$, i.e. for Dirichlet volume constraints. Specifically, they considered the problem of finding the optimal forcing term $f$ such that the nonlocal solution $u$ is as close as possible to a given target function $\widehat{u}$, that is, we have that $\mu=f, \mathcal{M}$ is the identity operator, and $\|\cdot\|_{W}=\|\cdot\|_{L^{2}(\Omega)}$. As a result, we have the functional

$$
\mathcal{J}(u ; f)=\frac{1}{2}\|u-\widehat{u}\|_{L^{2}(\Omega)}^{2}+\frac{\beta}{2}\|f\|_{L^{2}(\Omega)}^{2} .
$$

There are no additional constraints on the solution, so the optimization is solely constrained by the nonlocal diffusion equation. The well-posedness of that equation is sufficient to guarantee the existence and uniqueness of an optimal pair $\left(u^{*}, f^{*}\right)$. Furthermore, D'Elia and Gunzburger (2014) showed that in the limit of vanishing nonlocality, i.e. as $\delta \rightarrow 0$, the optimal nonlocal state and control converge to the optimal solution $\left(u_{l}^{*}, f_{l}^{*}\right)$ of the local counterpart of (16.1) given as follows:

Seek $u_{l}(\boldsymbol{x}) \in H^{1}(\Omega)$ and $f_{l} \in L^{2}(\Omega)$ such that

$$
\begin{gathered}
\min _{f_{l} \in L^{2}(\Omega)} \mathcal{J}\left(u_{l} ; f_{l}\right) \\
\text { subject to } \begin{cases}-\Delta u_{l}=f_{l} & \text { for all } \boldsymbol{x} \in \Omega, \\
u_{l}=g & \text { for all } \boldsymbol{x} \in \partial \Omega .\end{cases}
\end{gathered}
$$




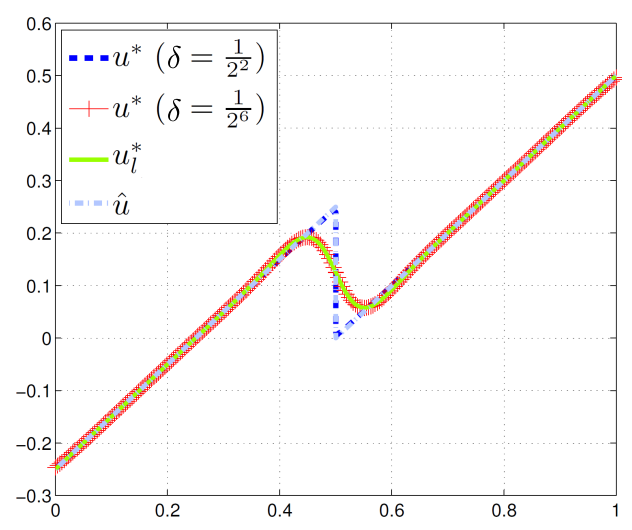

(a)

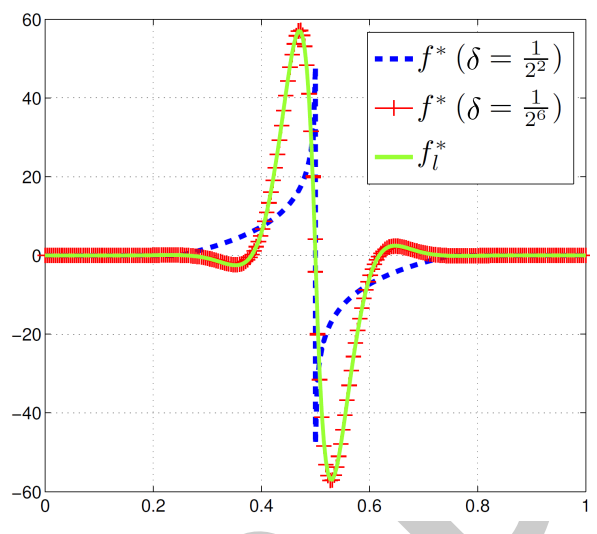

(b)

Figure 16.1. (a) The optimal nonlocal state $u^{*}$ for $\delta=2^{-2}$ and $2^{-6}$, the target function $\widehat{u}$, and the optimal local state $u_{l}^{*}$. (b) The corresponding optimal source terms.

For finite element discretizations of the state and control variables, D'Elia and Gunzburger (2014) proved the convergence of both variables with respect to $\delta$ and the mesh size $h$, along with error estimates. Also, numerical results for discontinuous target functions show that nonlocal models, for which irregular solutions are admissible, allow one to match non-smooth functions in a much better way compared to local models.

An example is provided in Figure 16.1 for the domains $\Omega=(0,1)$ and $\Omega_{\mathcal{I}_{\delta}}=(-\delta, 0) \cup(1,1+\delta)$, and for a target function having a jump discontinuity at $x=0.5$. Discontinuous piecewise linear finite element discretizations are used for both the state and control. For comparison purposes, continuous piecewise linear finite element approximations of the local optimal control problem (16.5) are also computed. In Figure 16.1(a) we plot the target function $\widehat{u}$, the optimal local state $u_{l}^{*}$ (the solution of (16.5)) and nonlocal optimal state $u^{*}$ (for two values of $\delta$ ). Note that for a large horizon $\delta$ the nonlocal solution perfectly matches the target, whereas for a small horizon the nonlocal optimal solution is visually identical to the local one. In Figure 16.1(b) we plot the corresponding optimal source terms $f^{*}$. Here, for a large horizon, the control has a smaller amplitude and a smaller $L^{2}$ norm (which can be viewed as indications of a smaller cost of control) even though that control does a better job of matching the target function. As explained in D'Elia and Gunzburger (2014), this behaviour can be justified by the fact that the nonlocal model allows for discontinuous behaviour in the optimal state, and thus the optimal control has an 'easier time' forcing a match between the optimal state and the non-smooth target function. 


\subsubsection{Coefficient identification}

The identification of kernel parameters or of the kernel function itself is one of the most important open problems in nonlocal modelling. In fact, in general, the choice of kernel function and its parameters is based on intuition or designed through heuristic techniques. Here we report on three approaches that tackle the kernel identification problem using different objective functionals.

Fuensanta and Muñoz (2015) considered the problem of identifying a constitutive function in (16.2) having the form $\theta_{\delta}(\boldsymbol{x}, \boldsymbol{y})=\vartheta(\boldsymbol{x})+\vartheta(\boldsymbol{y})$, that is, to identify the parameter function $\mu=\vartheta(\boldsymbol{x})$. In addition, $\mathcal{V} u=u$ and $\phi_{\delta}(\boldsymbol{x}, \boldsymbol{y})=k(\boldsymbol{x}, \boldsymbol{y})|\boldsymbol{y}-\boldsymbol{x}|^{-2}$ with $k(\boldsymbol{x}, \boldsymbol{y}) \geq C|\boldsymbol{y}-\boldsymbol{x}|^{2-d-2 s}$ for $s \in(0,1)$ and $\operatorname{support}(k(\boldsymbol{x}, \boldsymbol{y}))=B_{\delta}(\boldsymbol{x})$. The set of admissible $\vartheta(\boldsymbol{x})$ is defined as

$$
W=\left\{\vartheta(\boldsymbol{x}) \in\left[\vartheta_{\min }, \vartheta_{\max }\right], \vartheta=0 \text { on } \Omega_{\mathcal{I}}, \int_{\Omega} \vartheta(\boldsymbol{x}) \mathrm{d} \boldsymbol{x}=\bar{\vartheta}\right\}
$$

for positive constants $\vartheta_{\min }, \vartheta_{\max }$ and $\bar{\vartheta}$. The objective functional in Fuensanta and Muñoz (2015) consists of a cost functional only and it is referred to as a compliance functional; it is defined as

$$
\mathcal{J}(u)=\int_{\Omega \cup \Omega_{I}} \int_{\Omega \cup \Omega_{I}} F(\boldsymbol{x}, \boldsymbol{y} ; u) \mathrm{d} \boldsymbol{y} \mathrm{d} \boldsymbol{x}
$$

and is then minimized over $\vartheta(\boldsymbol{x}) \in W$. First, $F$ is chosen such that $\mathcal{J}(u)=\|u\|^{2}$, where $\|\cdot\| \|$ denotes the 'energy' norm corresponding to the kernel function $\phi(\boldsymbol{x}, \boldsymbol{y})=k(\boldsymbol{x}, \boldsymbol{y})|\boldsymbol{y}-\boldsymbol{x}|^{-2}$. For this choice of $F$ and with no other constraints applied on the state or control, the existence of a solution of the problem of minimizing the functional (16.6) is proved. Note that a regularizing term is not included in this functional because the box constraints on $\vartheta(\boldsymbol{x})$ included in the admissibility set $W$ already guarantee the well-posedness of the minimization problem. Extensions to more general functionals are then considered, including $F$ only being required to be measurable with respect to $u$ and lower semi-continuous with respect to $\boldsymbol{x}$. Furthermore, for the compliance case, the convergence of the optimal nonlocal solution to its local counterpart (16.5) for $\mu=\vartheta$ is proved. In this work, neither discretizations nor numerical tests are provided.

D'Elia and Gunzburger (2016) also considered the problem of identifying the constitutive function $\theta_{\delta}(\boldsymbol{x}, \boldsymbol{y})$ in (16.2) for square-integrable and also non-integrable kernel functions $\phi_{\delta}(\boldsymbol{x}, \boldsymbol{y})$ - specifically, again $\mathcal{V} u=u$, and, in the functional (16.3), $\beta=0$ and $\|\cdot\|_{U(\Omega)}=\|\cdot\|_{L^{2}(\Omega)}$. The functional is minimized over the set of admissible controls given by

$W=\left\{\theta_{\delta} \in W^{1, \infty}\left(\Omega \cup \Omega_{\mathcal{I}_{\delta}} \cup \Omega \cup \Omega_{\mathcal{I}_{\delta}}\right), \theta_{\delta} \in\left[\vartheta_{\min }, \vartheta_{\text {max }}\right],\|\theta\|_{1, \infty} \leq \theta_{\delta}^{\max }<\infty\right\}$.

Again, the box constraints on $\theta_{\delta}$ included in the admissibility set suffice to prove that problem (16.1) has at least one solution. D'Elia and Gunzburger 


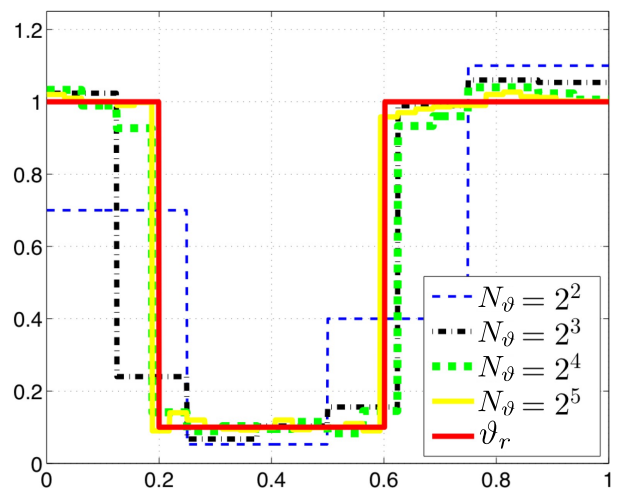

(a)

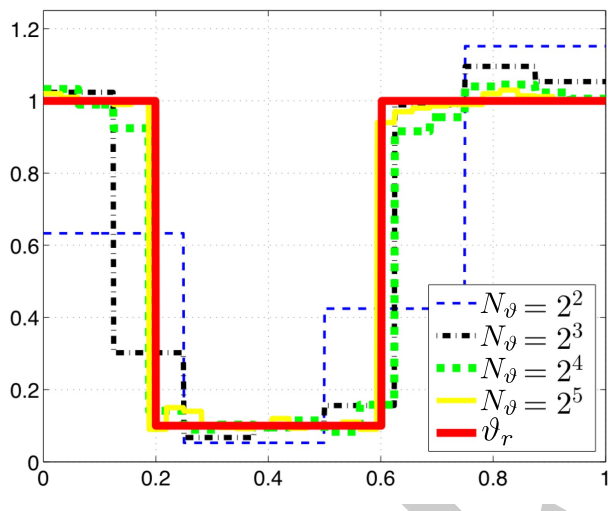

(b)

Figure 16.2. Optimal approximate coefficient functions for $\delta=2^{-9}$ (a) and $2^{-4}$ (b) for different numbers of degrees of freedom $N_{\vartheta}$ in the discretization of the coefficient function $\vartheta(z)$ defined in (16.7).

(2016) developed a mixed finite element discretization of the state and control variable and proved the convergence of the discretization error as the mesh size is refined. Numerical tests are also provided that show that the approach taken there allows for the identification of both smooth and discontinuous diffusion coefficients for both square-integrable and (truncated) fractional kernels. A sample result is shown in Figure 16.2. For that figure, $\Omega=(0,1), \Omega_{\mathcal{I}_{\delta}}=(\delta, 0) \cup(1,1+\delta), g(x)=0, f(x)=5$, and the spatial grid size used to discretize the state is $2^{-12}$. A surrogate for the target functional $\widehat{u}$ is a very fine-grid finite element approximation of the nonlocal diffusion problem with the data just listed and with

$$
\theta_{\delta}(x, y)=\vartheta\left(\frac{x+y}{2}\right) \quad \text { with } \vartheta(z)= \begin{cases}1 & \text { for all } z \in(0,0.2) \\ 0.1 & \text { for all } z \in(0.2,0.6) \\ 1 & \text { for all } z \in(0.6,1)\end{cases}
$$

Thus the goal of the minimization problem is to identify this constitutive coefficient function. Figure 16.2 illustrates, for two values of the horizon $\delta$, the convergence (with respect to the grid size $1 / N_{\vartheta}$ used to approximate the coefficient function $\vartheta(z)$ ) of the approximation.

We also mention the approach introduced in Pang, D'Elia, Parks and Karniadakis $(2019 a)$, in which the task of parameter identification for truncated diffusion operators of fractional type is pursued by including the nonlocal diffusion equation in the objective functional, that is, we have

$$
\mathcal{J}(u ; \boldsymbol{\mu})=\frac{1}{2}\|u-\widehat{u}\|_{L^{2}(\Omega)}^{2}+\frac{\beta}{2}\|-\mathcal{L} u-f\|_{L^{2}(\Omega)}^{2} .
$$


Thus the state equations are only weakly prescribed through the minimization of the residual. Pang et al. (2019a) approximated the state and control variables using fully connected neural networks. Numerical tests on analytic solutions and turbulence models show the ability of this technique to recover kernel parameters such as the interaction radius $\delta$ and the variable fractional order $s(\boldsymbol{x})$, that is, we have $\mu=\{\delta, s(\boldsymbol{x})\}$.

\subsection{Inverse problems for fractional operators}

In this section we focus on control and optimization problems for the fractional Laplace operator. The formulations described in what follows can be easily extended to more general fractional operators (see e.g. Meerschaert and Sikorskii 2012), but at a cost of more complicated analyses.

\subsubsection{Distributed optimal control in fractional diffusion for a matching functional}

The formulation presented in the previous section for distributed control can be used for fractional operators with (almost) no modification. We mention several works in the literature that analyse the theoretical and numerical aspects of both the elliptic and parabolic fractional problems.

D'Elia, Glusa and Otárola (2019b) considered the same problem as that introduced in Section 16.1.1. Specifically, for $\mathcal{J}(u ; f)$ as in $(16.4)$ and $\mathcal{V} u=$ $u$, they considered the problem of minimizing $\mathcal{J}(u ; f)$ with respect to $f \in$ $W=\left\{r \in L^{2}(\Omega): r \in\left[r_{\min }, r_{\max }\right]\right\}$ subject to

$$
\begin{cases}(-\Delta)^{s} u=f & \text { for all } \boldsymbol{x} \in \Omega, \\ u=g & \text { for all } \boldsymbol{x} \in \mathbb{R}^{d} \backslash \Omega .\end{cases}
$$

The well-posedness of the control problem is proved, optimality conditions are derived, and regularity estimates for the optimal variables are also proved. Furthermore, based on an a priori error analysis for the state equation, a semidiscrete scheme is constructed for which a priori error estimates for the approximation of the control variable are derived. A fully discrete scheme is also considered for which state and control error estimates are derived. Several two-dimensional numerical illustrations of the theoretical results are also provided.

Glusa and Otárola (2019), in a follow-up paper, considered the fractional parabolic equation (15.4). The objective functional, similar to that in (16.4), now involves an integral over time for both the cost and regularization terms, that is,

$$
\mathcal{J}(u ; f)=\frac{1}{2} \int_{0}^{T}\left(\|u-\widehat{u}\|_{L^{2}(\Omega)}^{2}+\beta\|f\|_{L^{2}(\Omega)}^{2}\right) \mathrm{d} t .
$$


Further, the control variable $f$ is now also time-dependent and belongs to the admissibility set

$$
W(t)=\left\{r(t) \in L^{2}(\Omega): r(t) \in\left[r_{\min }(t), r_{\max }(t)\right] \text { for all } t \in(0, T]\right\} .
$$

As in D'Elia et al. (2019b), the existence and uniqueness of optimal solutions are proved, and first-order necessary and sufficient optimality conditions are derived. Also derived are regularity estimates for the optimal state and control. Then discrete stability results and a priori error estimates are derived for the discretized problem resulting from the standard backward Euler scheme for temporal discretization and a piecewise linear finite element spatial discretization. The theoretical findings are illustrated by oneand two-dimensional numerical experiments.

Antil, Khatri and Warma (2019a) and Antil, Verma and Warma (2019b) chose the control as the data $g$ in the volume constraint for the steadystate and time-dependent cases, respectively. In (16.1), nonlocal Dirichlet, Neumann and Robin volume constraints are considered for the operator $\mathcal{V}$. As an example, in the Robin case we have

$$
\mathcal{V} u=\kappa_{N} \mathcal{N} u+\kappa_{D} u=g,
$$

where $\mathcal{N}$ is defined as in (1.10) with the appropriate changes made to reflect that here the interaction domain is $\Omega_{\mathcal{I}_{\infty}}=\mathbb{R}^{d} \backslash \Omega$. Even though the theory is presented for the general functional

$$
\mathcal{J}(u ; g)=\mathcal{Q}(u)+\frac{\beta}{2} \int_{0}^{T}\|g\|_{L^{2}\left(\Omega_{\mathcal{I}}\right)}^{2} \mathrm{~d} t
$$

with a convex cost functional $\mathcal{Q}(u)$, numerical experiments are performed for a matching functional in the usual form; see e.g. (16.10) for the timedependent version. In the more general formulation, for the time-dependent case, Antil et al. (2019b) mostly focus on nonlocal Dirichlet and Robin optimal control problems. Well-posedness and regularity are discussed and a discretization scheme is proposed. The theoretical results are illustrated by two-dimensional numerical experiments. The main contribution of Antil et al. $(2019 b)$ is to show the ability of nonlocal models to take advantage of information outside the domain and not only on the boundary, which is one of the limitations of control problems for PDEs.

Even if not entirely focused on operators such as that in (3.1), we mention that Antil and Warma (2020) consider control problems for both a spectral fractional semilinear operator and for the integral fractional Laplacian. They derive existence and regularity results for the spectral case (which is not treated in this section). They also state that the results obtained for the spectral case can be extended, after small modifications, to the integral definition of the operator. 
Remark 15.1 (kernel identification). For kernel identification in the setting of fractional operators, we should mention that Pang, Lu and Karniadakis $(2019 b)$ studied an algorithm for parameter identification based on physics-informed neural networks. As such, this method is a special instance of the algorithm presented in Pang et al. (2019a). Specifically, this work is focused on the estimation of the fractional power of the integral fractional Laplacian. Other works on the identification of kernel parameters, more specifically, of the fractional power $s$, only deal with the spectral definition of $(-\Delta)^{s} u$.

Remark 15.2 (control for the spectral fractional Laplacian). We should also mention that control of equations involving the spectral definition of the fractional Laplacian has been analysed; see e.g. Antil, Otarola and Salgado $(2016,2018)$ and Otárola and Salgado (2018).

\section{Variational inequalities and obstacle problems}

We consider the nonlocal obstacle problem

$$
\begin{cases}-\mathcal{L} u \geq f & \text { for all } \boldsymbol{x} \in \Omega \\ u \geq \psi & \text { for all } \boldsymbol{x} \in \Omega \\ (-\mathcal{L} u-f)(u-\psi)=0 & \text { for all } \boldsymbol{x} \in \Omega \\ u=0 & \text { for all } \boldsymbol{x} \in \Omega_{\mathcal{I}_{\infty}}\end{cases}
$$

where $\psi(\boldsymbol{x})$ denotes the obstacle function. Nonlocal obstacle problems such as this one are used in studying the deformation of elastic membranes, in contact mechanics and in finance, for example the pricing of American put options in Lévy jump-diffusion models. Clearly, (17.1) is a nonlocal analogue of the local PDE obstacle problem

$$
\begin{cases}-\Delta u \geq f & \text { for all } \boldsymbol{x} \in \Omega \\ u \geq \psi & \text { for all } \boldsymbol{x} \in \Omega \\ (-\Delta u-f)(u-\psi)=0 & \text { for all } \boldsymbol{x} \in \Omega \\ u=0 & \text { for all } \boldsymbol{x} \in \partial \Omega\end{cases}
$$

The well-posedness analysis of nonlocal obstacle problems needs less smooth obstacles than the corresponding local PDE obstacle problems. Moreover, as is illustrated at the end of this section, the behaviours and properties of solutions in the local and nonlocal setting can be quite different.

There is an ever-growing literature on the analysis and approximation of nonlocal obstacle problems, especially in the fractional setting. Here we give a brief account of an approach used by Burkovska and Gunzburger (2019b), who considered regularity estimates, well-posedness analyses, and finite element methods and their numerical analysis. Using a different 
approach, Guan and Gunzburger (2017) established the well-posedness of nonlocal obstacle problems and the convergence of the finite element approximation for fractional Laplacian kernels and for kernels that are both radial and integrable. With respect to other works about obstacle problems for the fractional Laplacian, we mention Servadei and Valdinoci (2013), who obtained Lewy-Stampacchia-type estimates similar to those obtained by Burkovska and Gunzburger (2019b), but with restrictions on the fractional exponent and requiring greater smoothness of the obstacle. The regularity of the obstacle problem measured in Hölder and Lipschitz spaces was studied by Silvestre (2017) and Caffarelli, Ros-Oton and Serra (2017), for example. Borthagaray, Nochetto and Salgado (2019) studied a finite element approximation of the obstacle problem for the fractional Laplacian, and proved error estimates. Regularity results for the solution are derived in weighted Sobolev spaces under additional regularity assumptions on the right-hand side (Hölder continuity) and the obstacle. Bonito, Lei and Salgado (2020) studied the regularity of the obstacle problem involving integro-differential operators, with the fractional Laplacian as the nonlocal term, and proposed and analysed a finite element-based discretization. In the purely nonlocal case, the same regularity for the solution is proved as in Burkovska and Gunzburger (2019b), but for more restricted cases. Other than Burkovska and Gunzburger (2019b), none of these works on the obstacle problem for the fractional Laplacian treat truncated kernels.

Burkovska and Gunzburger (2019b) obtained the well-posedness and regularity results for the nonlocal obstacle problem (17.1), and used the mixed formulation

$$
\begin{cases}\mathcal{A}(u, v)-\mathcal{B}(\lambda, v)=\langle f, v\rangle & \text { for all } v \in V \\ \mathcal{B}(\eta-\lambda, u-\psi) \geq 0 & \text { for all } \eta \in M \subset V_{d}\end{cases}
$$

to define, analyse and apply finite element methods. In $(17.2), \mathcal{A}(\cdot, \cdot): V \times$ $V \rightarrow \mathbb{R}$ is the usual bilinear form corresponding to the nonlocal operator $\mathcal{L}$, $V$ is the energy space associated with that bilinear form and the homogeneous volume constraint, $V_{d}$ is the dual space for $V, \mathcal{B}(\cdot, \cdot): V_{d} \times V \rightarrow \mathbb{R}$ is defined as $\mathcal{B}(\eta, v)=\langle\eta, v\rangle$, and $M$ denotes the closed convex dual cone

$$
M:=\left\{\eta \in V_{d}:\langle\eta, v\rangle \geq 0 \text { for all } v \in V, v \geq 0\right\} .
$$

Of course, $\mathcal{A}(\cdot, \cdot)$ is continuous and coercive in $V \times V$, and Burkovska and Gunzburger $(2019 b)$ showed that $\mathcal{B}(\cdot, \cdot)$ is continuous and inf-sup stable on $V_{d} \times V$, that is, we have

$$
\inf _{\eta \in V_{d}} \sup _{v \in V} \frac{\mathcal{B}(\eta, v)}{\|\eta\|_{V_{d}}\|v\|_{V}} \geq \beta_{0}>0 .
$$

So, the task at hand is to find, for $f \in V_{d}$ and $\psi \in V, u \in V$ and $\lambda \in M$ satisfying (17.2). 


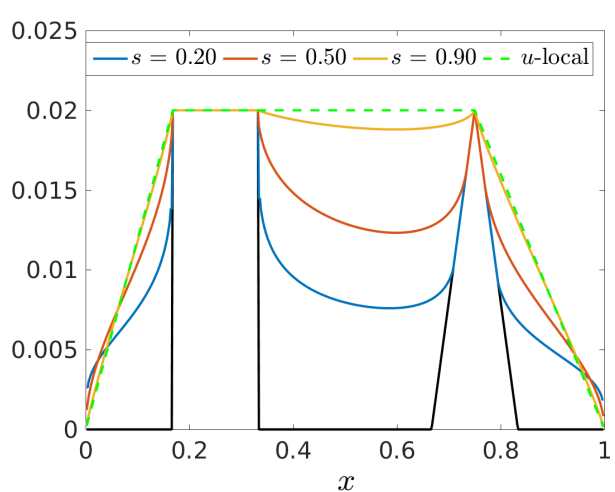

(a)

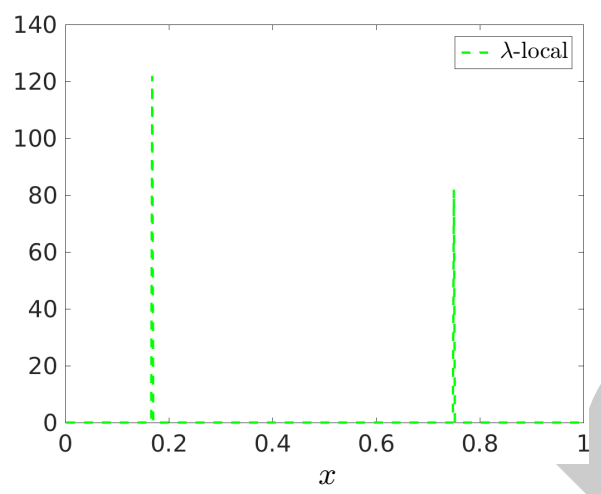

(c)

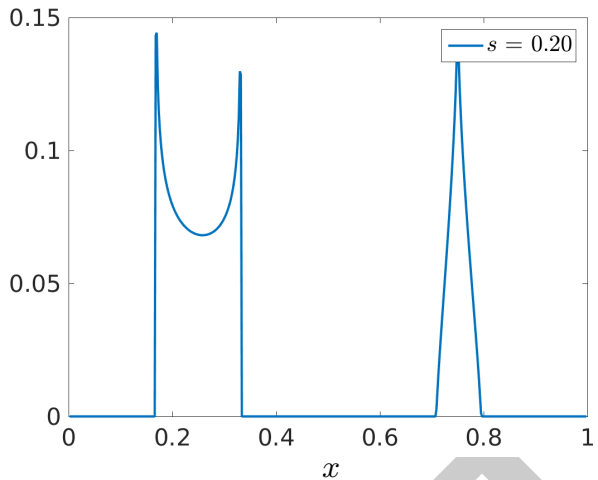

(b)

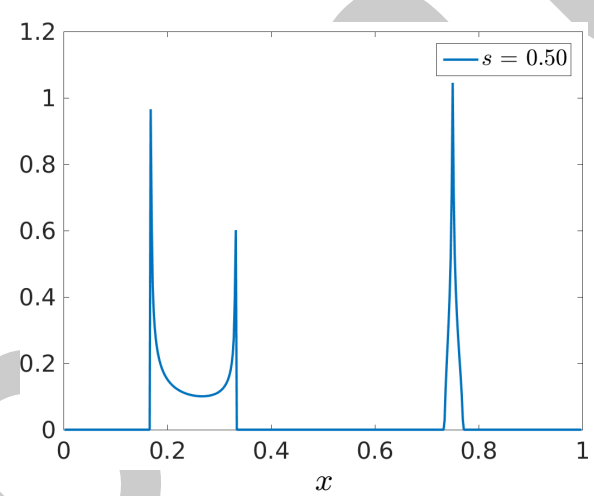

(d)

Figure 17.1. (a) Primal nonlocal solutions for three values of the fractional exponent and the primal local solution. (b) The local Lagrange multiplier. (c, d) The nonlocal Lagrange multiplier for $s=0.2(\mathrm{c})$ and $s=0.5(\mathrm{~b})$. Note the different ordinate scales in the three Lagrange multiplier plots.

Under the assumptions $f \in L^{2}(\Omega)$ and $\left.\left(-\mathcal{L}_{\delta} \psi-f\right)_{+}\right|_{\Omega} \in L^{2}(\Omega)$, the improved regularity results

$$
\mathcal{L}_{\delta} u \in L^{2}(\Omega), \quad \lambda \in L^{2}(\Omega), \quad \lambda \leq\left(-\mathcal{L}_{\delta} \psi-f\right)_{+}
$$

are derived. For the fractional Laplacian obstacle problem, the improved regularity results obtained are given by

$$
\lambda \in L^{2}(\Omega) \quad \text { and } \quad u \in H_{\Omega}^{s+\beta}(\Omega)
$$

with $\beta=\min \{s, 1 / 2-\varepsilon\}, \varepsilon>0, s \in(0,1)$. Note that these regularity results hold for all $s \in(0,1)$.

Finite element approximations are defined for the space $V^{h} \subset V$ of piecewise linear continuous polynomials and the space $V_{d}^{h} \subset V_{d}$ of discontinuous linear polynomials for which locally bi-orthogonal basis functions can be 
constructed, that is, for any basis functions $\xi_{j}(\boldsymbol{x}) \in V_{d}^{h}$ and $\phi_{j^{\prime}}(\boldsymbol{x}) \in V^{h}$ and for any finite element $K$, we have

$$
\int_{K} \xi_{j} \phi_{j^{\prime}}=\delta_{j j^{\prime}} \int_{K} \phi_{j} \geq 0 .
$$

The inf-sup stability with respect to $V_{d}^{h} \times V^{h}$ is proved, so the discrete problem is well-posed.

An example numerical result is provided in Figure 17.1. Note the differences in the primal solution $u(\boldsymbol{x})$ and in the Lagrange multiplier $\lambda$ obtained using nonlocal and local models. In particular, note that for the nonlocal case $\lambda \in L^{2}(\Omega)$, whereas for the local case $\lambda$ consists of Dirac delta functions.

Note that all the results in Burkovska and Gunzburger (2019b) pertinent to the fractional Laplacian were also proved for the truncated fractional Laplacian kernel introduced in Section 3.1.1. Moreover, the convergence of the solution of the obstacle problem with the truncated kernel is shown to converge to the solution of (the un-truncated) fractional Laplacian obstacle problem.

\section{Reduced-order modelling}

Reduced-order modelling (ROM) is the task of constructing a very low-dimensional discretization for parametrized problems that, in order to achieve a desired fidelity, are usually approximated by a high-dimensional discretization (HDD). The construction of a ROM usually requires an off-line cost incurred by having to do runs of the HDD for relatively few parameter choices. Once the ROM is constructed, it can be used on-line instead of the HDD by, for example, doing additional simulations for many more parameter choices at much lower cost than if the HDD were used instead. For example, in uncertainty quantification settings, one may need to obtain many simulations in order to obtain good statistical information, so using the ROM instead of the HHD can result in huge computational savings. The huge body of literature on ROMs for PDEs attests to their usefulness.

PDE discretizations ubiquitously involve large, sparse linear or nonlinear discrete systems. Analogous discretizations of nonlocal models usually involve discrete systems of similar size but, due to nonlocality, having much less sparsity. In some settings, such as those involving fractional Laplacians, the discrete systems may even be full. The double curse of highdimensionality and lack of sparsity of the discrete systems means that:

Reduced-order modelling is needed much more for nonlocal models than for corresponding local PDE models.

The setting we use to discuss ROMs is parametrized nonlocal diffusion. Thus we assume that the constitutive function $\theta_{\delta}\left(\boldsymbol{x}, \boldsymbol{y} ; \boldsymbol{p}_{\theta}\right)$ in $(2.16)$ depends on the components of a parameter vector $\boldsymbol{p}_{\theta} \in \Gamma_{\theta} \subset \mathbb{R}^{N_{\theta}}$, and the kernel 
function $\phi_{\delta}\left(\boldsymbol{x}, \boldsymbol{y} ; \boldsymbol{p}_{\phi}\right)$ in $(2.16)$ depends on the components of a parameter vector $\boldsymbol{p}_{\phi} \in \Gamma_{\phi} \subset \mathbb{R}^{N_{\phi}}$. We refer to $\Gamma_{\theta}$ and $\Gamma_{\phi}$ as parameter domains. Then, in the weak formulation (2.9) of the nonlocal diffusion problem, we have the bilinear form

$$
\begin{aligned}
& \mathcal{A}_{\boldsymbol{p}}(u, v) \\
& =\int_{\Omega \cup \Omega_{\mathcal{I}_{\delta}}} \int_{B_{\delta}(\boldsymbol{x})}(v(\boldsymbol{y})-v(\boldsymbol{x}))(u(\boldsymbol{y})-u(\boldsymbol{x})) \phi_{\delta}\left(\boldsymbol{x}, \boldsymbol{y} ; \boldsymbol{p}_{\phi}\right) \theta_{\delta}\left(\boldsymbol{x}, \boldsymbol{y} ; \boldsymbol{p}_{\theta}\right) \mathrm{d} \boldsymbol{y} \mathrm{d} \boldsymbol{x},
\end{aligned}
$$

where we have used $(2.16)$, i.e. $\gamma_{\delta}(\boldsymbol{x}, \boldsymbol{y})=\phi_{\delta}\left(\boldsymbol{x}, \boldsymbol{y} ; \boldsymbol{p}_{\phi}\right) \theta_{\delta}\left(\boldsymbol{x}, \boldsymbol{y} ; \boldsymbol{p}_{\theta}\right) \mathcal{X}_{B_{\delta}(\boldsymbol{x})}(\boldsymbol{y})$. We also assume that we have in hand a finite element or other variational discretization ${ }^{5}$ of $(2.9)$ with the bilinear form $\mathcal{A}_{\boldsymbol{p}}(\cdot, \cdot)$, that is, we have the nonlocal discrete problem for $u_{\delta, h, \boldsymbol{p}}(\boldsymbol{x}) \in V_{c}^{h}\left(\Omega \cup \Omega_{\mathcal{I}_{\delta}}\right)$ given by

$$
\mathcal{A}_{\boldsymbol{p}}\left(u_{\delta, h, \boldsymbol{p}}, v_{h}\right)=\left\langle f, v^{h}\right\rangle \quad \text { for all } v_{h} \in V_{c}^{h}\left(\Omega \cup \Omega_{\mathcal{I}_{\delta}}\right),
$$

where, for economy of exposition, we impose the homogeneous Dirichlet volume constraint $u_{\delta, h, \boldsymbol{p}}(\boldsymbol{x})=0$ on $\Omega_{\mathcal{I}_{\delta}}$ and where $V_{c}^{h}\left(\Omega \cup \Omega_{\mathcal{I}_{\delta}}\right)$ denotes a subspace of $V_{c}\left(\Omega \cup \Omega_{\mathcal{I}_{\delta}}\right)$, e.g. one generated via finite elements. Thus, given any parameter vectors $\boldsymbol{p}_{\phi} \in \Gamma_{\phi}$ and $\boldsymbol{p}_{\theta} \in \Gamma_{\theta}$, (18.1) can be solved to determine the approximation $u_{\delta, h, \boldsymbol{p}}(\boldsymbol{x})$ of the exact solution $u_{\delta, \boldsymbol{p}}(\boldsymbol{x})$ of (2.9) with $\mathcal{A}(\cdot, \cdot)$ replaced by $\mathcal{A}_{\boldsymbol{p}}(\cdot, \cdot)$. We let $N_{h}$ denote the dimension of $V_{c}^{h}\left(\Omega \cup \Omega_{\mathcal{I}_{\delta}}\right)$, i.e. of the HDD.

Assume we have a ROM basis $V_{N_{\text {rom }}}:=\left\{v_{n, \text { rom }}(\boldsymbol{x})\right\}_{n=1}^{N_{\text {rom }}}$ consisting of $N_{\text {rom }}$ functions $v_{n, \text { rom }}(\boldsymbol{x}) \in V_{c}^{h}\left(\Omega \cup \Omega_{\mathcal{I}_{\delta}}\right)$. Then, for any $\boldsymbol{p}_{\phi} \in \Gamma_{\phi}$ and $\boldsymbol{p}_{\theta} \in \Gamma_{\theta}$, the $R O M$-Galerkin approximation $u_{\boldsymbol{p} \text {,rom }}(\boldsymbol{x}) \in V_{N_{\mathrm{rom}}}$ of the solution $u_{\delta, h, \boldsymbol{p}}(\boldsymbol{x})$ of (18.1) is determined by solving the problem

$$
\mathcal{A}_{\boldsymbol{p}}\left(u_{\boldsymbol{p}, \mathrm{rom}}, v\right)=\langle f, v\rangle \text { for all } v \in V_{N_{\text {rom }}} .
$$

Thus the task at hand is to construct a ROM basis such that $N_{\text {rom }} \ll$ $N_{h}$, which results in ROM solutions $u_{\boldsymbol{p} \text {,rom }}(\boldsymbol{x})$ that are acceptably accurate approximations of $u_{\delta, h, \boldsymbol{p}}(\boldsymbol{x})$ for any $\boldsymbol{p}_{\phi} \in \Gamma_{\phi}$ and $\boldsymbol{p}_{\theta} \in \Gamma_{\theta}$, or at least for subsets of the parameter domains that are of interest.

Two examples of the use of reduced-order models for nonlocal diffusion are given by Guan, Gunzburger, Webster and Zhang (2017), who use a greedy reduced basis (GRB) approach, and Witman, Gunzburger and Peterson (2017), who use a proper orthogonal decomposition (POD) approach. ${ }^{6}$ Both approaches involve only the parameter vector $\boldsymbol{p}_{\theta}$. The basis construction

${ }^{5}$ ROMs for discretizations of the strong form of nonlocal models are also of interest.

6 Although both ROMs we consider, like most others, involve a 'reduced basis', i.e. a basis of lower dimension than that of the parent HDD model, reduced-basis methods usually refer to ROMs in which the basis consists of solutions of (18.2). In contrast, a POD basis consists of linear combinations of such solutions. 


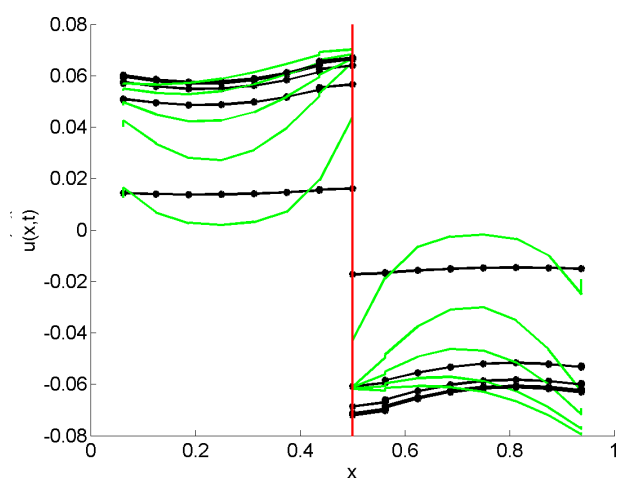

(a)

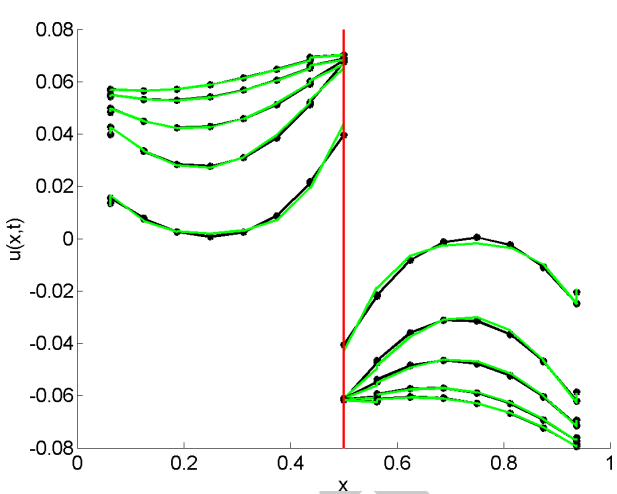

(b)

Figure 18.1. Comparison at different times between a fine-grid discontinuous Galerkin finite element approximation (green line) and POD approximations (black line with black dots) with one POD basis function (a) and six POD basis functions (b).

and application processes for nonlocal and local models are the same, excepting of course that in the local case approximate solutions of PDEs are involved whereas the nonlocal case involves approximate solutions of nonlocal models such as (18.2). Thus, here, we simply refer to Guan et al. (2017) and Witman et al. (2017) for detailed descriptions of the GRB and POD approaches, respectively, in the context of nonlocal diffusion models. Witman et al. (2017) considered a time-dependent problem. Guan et al. (2017) considered random parameter vectors and compared the GRB surrogates to sparse-grid surrogates. In addition to the analyses of the errors incurred by the ROMs, Guan et al. (2017) and Witman et al. (2017) provide numerical examples that illustrate the usefulness of the ROMs considered therein.

Burkovska and Gunzburger (2019a) considered the construction and analysis of ROMs for nonlocal models parametrized by the horizon $\delta$ and, for fractional models, by the fractional exponent $s$, so that now the parameter vector $\boldsymbol{p}_{\phi}$ is present in the kernel function. The parameter domains for both $\delta$ and $s$ are intervals bounded away from the origin and infinity, and greedy algorithms are used to construct the reduced bases. Also provided in Burkovska and Gunzburger (2019b) are illustrative numerical examples.

A very simple one-dimensional, time-dependent illustration of the effectiveness of ROM in the nonlocal setting is given in Figure 18.1. The kernel function is proportional to $1 /|y-x|$, so it is singular and non-integrable. A manufactured solution that has a jump discontinuity at all times is used to set the data of the problem. A discontinuous finite element Galerkin method is used both for generating the snapshots for the POD basis construction and for comparison purposes. Plots for five time instants are provided. From 
the figure, it is evident that a one-dimensional POD basis does a poor job of approximating the HDD finite element solution whereas a six-dimensional POD basis does an excellent job.

Remark 18.1. As is the case for the local PDE setting, obtaining solutions of nonlocal ROM models such as (18.2) incurs costs that depend only on the dimension $N_{\text {rom }}$ of the ROM model, whereas the naive assembly of the ROM models involves steps whose cost depends on the dimension $N_{h}$ of the HDD models used to generate the ROM bases. In the PDE setting, several strategies have been developed to overcome this obstacle, all of which can be applied to the nonlocal setting.

\section{A turbulent flow application}

A fundamental problem of fluid flows is to ascertain information about the paths taken by two initially closely positioned particles, in particular what happens, over a long time period, to the separation between a pair of particles. Such knowledge is crucial, for example, to predicting how pollutants spread. Intuitively, over a long time period, for laminar flows one expects that the separation between initially close particle pairs remains 'small' and, in fact, the mean-square separation is proportion to $t$. Also intuitively, over a long time period, for turbulent flows one expects that at least some initially close particle pairs may become widely separated. What happens to the separation between pairs of particles in turbulent flows is the issue addressed in the classic paper by Richardson (1926). This subject, often referred to as Richardson pair dispersion, has remained of interest to the present day. Reviews on the subject of Richardson pair dispersion are provided in Salazar and Collins (2009) and Swaford (2001).

Using dimensional analysis arguments, Richardson predicted that the mean-square separation in turbulent flows is proportional to $t^{3}$. Although it is generally accepted that the mean-square separation is not proportional to $t$ and that it grows faster than that, there is some controversy about the exponent 3, referred to as the Richardson constant. For example, a $t^{2}$ dependence is advocated by Bourgoin et al. (2006). Dispersion faster than $t$ is often referred to as superdiffusion. As discussed in Section 3, the fractional Laplace operator, when substituted for the classical Laplacian, is known to result in superdiffusive spread in 'parabolic' equations. Gunzburger, Jiang and $\mathrm{Xu}$ (2018) studied a modification of the Navier-Stokes equations introduced in Chen (2006), where the modification is that a fractional Laplacian viscous term is added to the standard Laplacian viscosity term.

The model considered is given by

$$
\begin{cases}\boldsymbol{u}_{t}+(\boldsymbol{u} \cdot \nabla) \boldsymbol{u}-\nu \Delta \boldsymbol{u}+\sigma(-\Delta)^{s} \boldsymbol{u}+\nabla p=\boldsymbol{f} & \text { in }(0, T] \times \Omega, \\ \nabla \cdot \boldsymbol{u}=0 & \text { in }(0, T] \times \Omega,\end{cases}
$$


where $s \in(0,1), \Omega \subset \mathbb{R}^{d}$ denotes a bounded, open domain, $\sigma$ denotes a constant and $[0, T]$ denotes a temporal interval of interest.

Gunzburger et al. (2018) have investigated the energy spectrum of the modified Navier Stokes equations (19.1). They have shown that for the special value of the fractional exponent $s=1 / 3$, the corresponding power law of the energy spectrum in the inertial range has a deviation from the well-known Kolmogorov $-5 / 3$ scaling, that is, instead of a $k^{-5 / 3}$ decay in the spectrum, one has $k^{-5 / 3+\beta}$ for a constant $\beta$, where $k$ denotes the frequency. For other values of $s \in(0,1)$, the power law of the energy spectrum is consistent with Kolmogorov's theory.

An IMEX scheme can be used to discretize the system (19.1); a backwardEuler scheme of that type is considered in Gunzburger et al. (2018) and is proved to be unconditionally stable and first-order accurate. Both the usual viscous term $\nu \Delta \boldsymbol{u}$ and the added fractional viscous term $\sigma(-\Delta)^{s} \boldsymbol{u}$ are treated implicitly. Because of the implicit treatment of the fractional Laplacian term, the scheme requires the solution of a dense linear system at each time step. Having to also handle the standard Navier-Stokes terms makes for an even greater computational challenge. Thus it is tempting to lag the fractional term to the previous time step. Unfortunately, this leads to serious stability issues, so that term has to be treated implicitly. To mitigate these challenges, the following two-stage operator splitting algorithm is introduced in Gunzburger et al. (2018).

Stage 1. Given $u^{n}$, find $w^{n+1}$ satisfying

$$
\left\{\begin{array}{l}
\frac{\boldsymbol{w}^{n+1}-\boldsymbol{u}^{n}}{\Delta t}+\left(\boldsymbol{u}^{n} \cdot \nabla\right) \boldsymbol{w}^{n+1}-\nu \Delta \boldsymbol{w}^{n+1}+\nabla p^{n+1}=\boldsymbol{f}^{n+1}-\sigma(-\Delta)^{\alpha} \boldsymbol{u}^{n}, \\
\nabla \cdot \boldsymbol{w}^{n+1}=0 .
\end{array}\right.
$$

Stage 2. Given $\boldsymbol{u}^{n}$ and $\boldsymbol{w}^{n+1}$, find $\boldsymbol{u}^{n+1}$ satisfying

$$
2 \Delta t \sigma(-\Delta)^{\alpha}\left(\boldsymbol{u}^{n+1}-u^{n}\right)+\boldsymbol{u}^{n+1}-\boldsymbol{w}^{n+1}=0 .
$$

The Stage 1 problem can be solved using a legacy Navier-Stokes code with the only modification necessary being in the construction of the right-hand side. In the second stage we solve a nonlocal Poisson problem for the fractional Laplace operator which involves a symmetric, positive definite, albeit dense linear system. This two-stage algorithm, although involving two linear system solves per time step, when compared to the algorithm in which the two viscous terms are treated monolithically, requires much less coding effort and introduces efficiencies not possible for the monolithic scheme.

Of course, we have glossed over the fact that the fractional Laplacian involves an integral over all of $\mathbb{R}^{d}$. Moreover, we also have to choose a spatial discretization scheme. For the first of these, we can, for example, 
use the truncated approximation discussed in Section 3.1.1, whereas for the latter we can use a finite element method based on the Taylor-Hood finite element pair (Girault and Raviart 1986), for example. Such fully discrete schemes involving the two-stage time-stepping algorithm have proved to be unconditionally stable and satisfy the error estimate

$$
\left\|u_{\infty}\left(t^{n}\right)-u_{h, \delta}^{n}\right\|_{L^{2}(\Omega)} \leq C\left(\frac{1}{\delta^{2 s}}+h^{3}+\Delta t\right),
$$

where $u\left(t^{n}\right)$ denotes the exact solution evaluated at time $t=t_{n}$ and with no truncation of the fractional Laplace operator involved, $u_{h, \delta}^{n}$ denotes the approximate solution, and $h$ and $\Delta t$ denote spatial and temporal grid size parameters, respectively. Note that for $s=1 / 3$ we have that $1 / \delta^{2 s}=1 / \delta^{2 / 3}$.

Remark 19.1 (another fractional turbulence model). We point out that a similar fractional model for turbulence has been introduced in Song and Karniadakis (2018); here, the authors identify a universal form of fractional order that holds for any Reynolds number. The same model is further investigated in Pang et al. (2019a) in the context of fractional parameter identification; see Section 16.1.

\section{Peridynamics models for solid mechanics}

In Sections 1-3 we considered nonlocal models for scalar-valued functions that are appropriate for modelling nonlocal diffusion problems, for example. To provide an example of a nonlocal model for vector-valued functions, in this section we consider a peridynamics model for the vector-valued displacement function in solid mechanics. Peridynamics is a nonlocal, continuum model that has been shown to provide an effective means for nucleating and propagating defects such as fractures $(\mathrm{Hu}, \mathrm{Ha}$ and Bobaru 2012, Lipton 2014, Lipton 2017, Bobaru and Zhang 2015, Du, Tao and Tian 2018c).

The particular model we consider is the linear state-based peridynamics model for solid mechanics, introduced and analysed in Mengesha and Du (2014), that is a nonlocal analogue of the classical Navier equations of linear elasticity. The model of Mengesha and Du (2014) is a generalization of the peridynamics model introduced in Silling et al. (2007) and Silling (2010) when specialized to linear constitutive laws. The model of Mengesha and Du (2014) is defined via an energy minimization principle for which the corresponding Euler-Lagrange equation provides a weak formulation of the problem. It should be noted that although the model of Mengesha and Du (2014) generalizes the models in Silling et al. (2007) and Silling (2010), those earlier models are ubiquitous in peridynamic computations. Additionally, it should be noted that the models of Silling et al. (2007) and Silling (2010) cannot be cast in terms of fractional kernels. 
To define the model considered, we need to introduce the operators ${ }^{7} \mathcal{D}_{\delta}^{*}$ and $\mathcal{D}_{\delta, \omega}^{*}$, which are respectively defined via their action on a vector-valued function $\boldsymbol{u}(\boldsymbol{x})$ by

$$
\left(\mathcal{D}^{*} \boldsymbol{u}\right)(\boldsymbol{x}, \boldsymbol{y}):=-(\boldsymbol{u}(\boldsymbol{y})-\boldsymbol{u}(\boldsymbol{x})) \otimes \frac{\boldsymbol{y}-\boldsymbol{x}}{|\boldsymbol{y}-\boldsymbol{x}|}
$$

and

$$
\left(\mathcal{D}_{\delta, \omega}^{*} \boldsymbol{u}\right)(\boldsymbol{x}):=\int_{\Omega}\left(\mathcal{D}^{*} \boldsymbol{u}\right)(\boldsymbol{x}, \boldsymbol{y}) \omega_{\delta}(\boldsymbol{x}, \boldsymbol{y}) \mathrm{d} \boldsymbol{y}
$$

where

$$
\omega_{\delta}(\boldsymbol{x}, \boldsymbol{y}):=\frac{d}{m_{\delta}(\boldsymbol{x})} \gamma_{\delta}(|\boldsymbol{y}-\boldsymbol{x}|)|\boldsymbol{y}-\boldsymbol{x}|
$$

and

$$
m_{\delta}(\boldsymbol{x}):=\int_{\Omega} \gamma_{\delta}(|\boldsymbol{y}-\boldsymbol{x}|)|\boldsymbol{y}-\boldsymbol{x}|^{2} \mathrm{~d} \boldsymbol{y}
$$

We define the energy space

$$
V_{p, \delta}=\left\{\boldsymbol{u} \in\left[L^{2}\left(\Omega \cup \Omega_{\mathcal{I}_{\delta}}\right)\right]^{d}:\|\boldsymbol{u}\|_{V_{p, \delta}}<\infty,\left.\boldsymbol{u}\right|_{\Omega_{\mathcal{I}_{\delta}}}=0\right\}
$$

equipped with the norm

$$
\|\boldsymbol{u}\|_{V_{p, \delta}}=\int_{\Omega \cup \Omega_{\mathcal{I}_{\delta}}} \int_{\Omega \cup \Omega_{\mathcal{I}_{\delta}}} \gamma_{\delta}(|\boldsymbol{y}-\boldsymbol{x}|)\left(\operatorname{Tr}\left(\mathcal{D}^{*} \boldsymbol{u}\right)(\boldsymbol{x}, \boldsymbol{y})\right)^{2} \mathrm{~d} \boldsymbol{y} \mathrm{d} \boldsymbol{x},
$$

where $\operatorname{Tr}(\cdot)$ denotes the trace operator acting on a tensor. Also, for functions $\boldsymbol{u}(\boldsymbol{x}) \in V_{p, \delta}$ and $\boldsymbol{v}(\boldsymbol{x}) \in V_{p, \delta}$, we define the symmetric bilinear form

$$
\begin{aligned}
\mathcal{P}_{\delta}(\boldsymbol{u}, \boldsymbol{v}):= & \int_{\Omega}\left(\left(k-\frac{\alpha m_{\delta}(\boldsymbol{x})}{d^{2}}\right) \operatorname{Tr}\left(\mathcal{D}_{\delta, \omega}^{*} \boldsymbol{u}\right)(\boldsymbol{x}) \operatorname{Tr}\left(\mathcal{D}_{\delta, \omega}^{*} \boldsymbol{v}\right)(\boldsymbol{x})\right. \\
& \left.+\alpha \int_{\Omega} \gamma_{\delta}(|\boldsymbol{y}-\boldsymbol{x}|) \operatorname{Tr}\left(\mathcal{D}^{*} \boldsymbol{u}\right)(\boldsymbol{x}, \boldsymbol{y}) \operatorname{Tr}\left(\mathcal{D}^{*} \boldsymbol{v}\right)(\boldsymbol{x}, \boldsymbol{y}) \mathrm{d} \boldsymbol{y}\right) \mathrm{d} \boldsymbol{x},
\end{aligned}
$$

where $k$ and $\alpha$ denote scalar constants related to the bulk and shear modulus of the material.

${ }^{7}$ In (1.15), the operator $\mathcal{D}_{\delta}^{*}$ is defined via its action on a scalar-valued function $u(\boldsymbol{x})$, resulting in $\mathcal{D}_{\delta}^{*} u$ being a vector-valued function of $\boldsymbol{x}$ and $\boldsymbol{y}$. In (20.1), $\mathcal{D}_{\delta}^{*}$ is defined via its action on a vector-valued function $\boldsymbol{u}(\boldsymbol{x})$, resulting in $\mathcal{D}_{\delta}^{*} \boldsymbol{u}$ being a tensor-valued function of $\boldsymbol{x}$ and $\boldsymbol{y}$. This is entirely analogous to the local case in which $\nabla u$ is a vector and $\nabla \boldsymbol{u}$ is a tensor. 
With the notation established, the weak formulation of the peridynamics problem considered is given as follows:

$$
\begin{aligned}
& \text { Seek } \boldsymbol{u}(\boldsymbol{x}) \in V_{p, \delta} \text { such that } \boldsymbol{u}(\boldsymbol{x})=\mathbf{0} \text { on } \Omega_{\mathcal{I}_{\delta}} \text { and } \\
& \qquad \mathcal{P}_{\delta}(\boldsymbol{u}, \boldsymbol{v})=(\boldsymbol{f}, \boldsymbol{v}) \text { for all } \boldsymbol{v} \in V_{p, \delta} \\
& \text { with } \boldsymbol{v}(\boldsymbol{x})=\mathbf{0} \text { on } \Omega_{\mathcal{I}_{\delta}},
\end{aligned}
$$

where $(\cdot, \cdot)$ denotes the $\left[L^{2}(\Omega)\right]^{d}$ inner product. For simplicity, we have imposed a homogeneous volume constraint on the displacement $\boldsymbol{u}$. Nonlocal traction constraints and inhomogeneous volume constraints can also be treated.

Mengesha and Du (2014) established the coercivity and continuity of the bilinear form $\mathcal{P}_{\delta}(\cdot, \cdot)$ with respect to the energy space $V_{p, \delta}$, so the well-posedness of problem (20.3) can be rigorously established via the LaxMilgram theorem. Additional discussions, including studies with other types of nonlocal constraints, can be found in Mengesha and Du $(2015,2016)$ and Du (2019).

Remark 20.1. Some choices for the kernel function $\gamma_{\delta}(|z|)$ are those with bounded second moments, including those that are comparable to $|\boldsymbol{z}|^{-d-2 s}$ in the sense that they have the same singular behaviour at the origin as that of the fractional kernel (2.22). Of particular interest in the peridynamics setting are kernel functions that are both radial and integrable and also fractional kernels with $s<1 / 2$ because the corresponding function spaces in which the peridynamics model is well-posed admit functions with jump discontinuities. As such, discontinuities in the displacement $\boldsymbol{u}(\boldsymbol{x})$ can be viewed as fracture. Functions comparable to $|\boldsymbol{z}|^{-1}$ are integrable for $d \geq 2$ and are the ubiquitous choice made in peridynamics modelling, starting with the works of Silling (2000), Silling et al. (2007) and Silling (2010), in which peridynamics was first introduced.

The strong formulation corresponding to the weak formulation (2.9) has also been derived by Mengesha and Du (2014). In the local limit, i.e. as $\delta \rightarrow 0$, the weak solutions of the nonlocal state-based peridynamic model with an appropriately chosen kernel converge to that of the classical NavierCauchy equation of linear elasticity.

Remark 20.2 (bond breaking). There is more to peridynamics than just the weak and strong formulations introduced above. Certainly, as discussed in Remark 20.1, those models admit solutions having jump discontinuities over co-dimension 1 interfaces. For example, for kernels $\gamma_{\delta}(\boldsymbol{z})$ that are both radial and integrable, solutions are no smoother than the data so that, in general, discontinuities in the boundary data or in the source term will result in discontinuities in the solution. However, in the time-dependent setting, fracture and other defects can arise, for example due to large tensile loads, 
even if the initial condition data and other data are smooth. Thus, to model fracture nucleation, the peridynamics model equations, whether in weak or strong form, have been supplemented by a bond-breaking rule in some cases. Basically, such rules state that two points $\boldsymbol{x}$ and $\boldsymbol{y}$ that are initially bonded, i.e. within a distance $\delta$ from each other, become un-bonded if at a later time those points become separated by a distance greater than $\delta$. Bond-breaking rules and how they effect the nucleation of cracks are more complicated than the simplistic description just given, but further discussion is beyond the scope of this article. Detailed explanations may be found in Silling (2000), Lipton $(2014,2017)$, Lipton, Lehoucq and Sha (2018) and Du et al. (2018c), for example.

For additional detailed discussions about the material presented in this section, see e.g. Du et al. (2012a), Mengesha and Du (2016) and Du (2019), as well as Mengesha and Du (2014).

Numerical methods for both the strong and weak formulations of the peridynamics model have been developed. For example, consider the finite element methods discussed in Section 5. Let $\left\{V_{p, \delta, h}\right\} \subset V_{p, \delta}$ denote a family of finite element subspaces, where $h$ characterizes the mesh size, and for any $\boldsymbol{v} \in V_{p, \delta}$ we have a family of elements $\left\{\boldsymbol{v}_{h} \in V_{p, \delta, h}\right\}$ such that $\left\|\boldsymbol{v}_{h}-\boldsymbol{v}\right\|_{V_{p, \delta}} \rightarrow$ 0 as $h \rightarrow 0$ for any fixed $\delta>0$. For the finite element discretization of the state-based linear peridynamic model (20.3), we merely replace $V_{p, \delta}$ with $V_{p, \delta, h}$ in that equation to arrive at the following discrete problem:

$$
\text { Find } \boldsymbol{u}_{\delta, h} \in V_{p, \delta, h} \text { such that } \mathcal{P}_{\delta}\left(\boldsymbol{u}_{\delta, h}, \boldsymbol{v}\right)=(\boldsymbol{f}, \boldsymbol{v})_{L^{2}} \text { for all } \boldsymbol{v} \in V_{p, \delta, h} .
$$

The analysis of solutions of this problem can be formulated within the general framework of the AC schemes originally presented in Tian and Du (2014). It is rigorously shown in Tian and Du (2014) that, for linear multidimensional state-based peridynamic systems associated with (20.3), all conforming Galerkin approximations of the nonlocal models containing continuous piecewise linear functions are automatically AC. This means that they can recover the correct local limit as long as both $\delta$ and $h$ are diminishing, even if the nonlocality measure $\delta$ is reduced at a much faster pace than the mesh spacing $h$.

As was the case for the nonlocal diffusion case, if $h=o(\delta)$ as $\delta \rightarrow 0$, then we are able to obtain the correct local limit even for discontinuous piecewise constant finite element approximations when they are of the conforming type. Practically speaking, this implies that a mild growth of bandwidth in the finite element stiffness matrix is needed as the mesh is refined, in order to recover the correct local limit for Riemann sum-like quadratures or piecewise constant finite element schemes. On the other hand, if a constant bandwidth 
is kept as the mesh is refined, as is often practised in the peridynamics community, approximations may converge to an incorrect local limit.

\section{Image denoising}

We describe the use of nonlocal diffusion operators in a variational setting for image denoising. In this context, by considering intensity patterns in neighbourhoods of points surrounding a pixel, nonlocal filters allow for simultaneous conservation of structures (patterns) and textures. Classical methods, which use differential operators, do not necessarily guarantee feature preservation because, by definition, they only consider infinitesimal neighbourhoods around points.

Even though nonlocal-type filters have been used for decades, we refer to Buades, Coll and Morel (2010) as the first foundational work that can be related to a nonlocal diffusion equation as presented in this article. In that paper, the nonlocal-means (NL-means) filter for image denoising is proposed. Given a blurred (or noisy) image $f$ defined by its intensity in the image domain $\Omega$, the NL-means filter is defined as

$$
N L[f](\boldsymbol{x})=\frac{1}{c(\boldsymbol{x})} \int_{\Omega} \mathrm{e}^{-d_{a}(f(\boldsymbol{x}), f(\boldsymbol{y})) / D^{2}} f(\boldsymbol{y}) \mathrm{d} \boldsymbol{y} \quad \text { for all } \boldsymbol{x} \in \Omega
$$

where

$$
d_{a}(f(\boldsymbol{x}), f(\boldsymbol{y}))=\int_{\Omega} G_{a}(\boldsymbol{z})|f(\boldsymbol{x}+\boldsymbol{z})-f(\boldsymbol{y}+\boldsymbol{z})| \mathrm{d} \boldsymbol{z},
$$

with $G_{a}(\boldsymbol{z})$ a Gaussian with standard deviation $a$ and with $c(\boldsymbol{x})$ a normalizing factor. Note that the nonlocal filter is directly applied to the blurred image, that is, the nonlocal operator is not associated with a diffusion equation.

The variational interpretation of such a filter was introduced by Kindermann, Osher and Jones (2005), who interpreted the NL-means and other neighbourhood filters as regularizing functionals. Specifically, they formulated the denoising problem as an unconstrained optimization problem where the objective is to minimize the nonlocal functional

$$
\mathcal{J}_{R} u=\int_{\Omega} \int_{\Omega} g\left(\frac{|u(\boldsymbol{x})-u(\boldsymbol{y})|}{D^{2}}\right) \nu(|\boldsymbol{x}-\boldsymbol{y}|) \mathrm{d} \boldsymbol{y}+F(u, f),
$$

where $\nu$ is a symmetric window that determines the extent of the nonlocal interactions and $g$ determines the type of filtering. Here, $F(u, f)$ is a fidelity term, usually a measure of the distance between the reconstructed and noisy images. The outcome of the optimization is the reconstructed image $u$. For 
NL-means filtering, (21.2) becomes

$$
\mathcal{J}_{N L} u=\int_{\Omega} \int_{\Omega}\left(1-\mathrm{e}^{-d_{a}(u(\boldsymbol{x}), u(\boldsymbol{y})) / D^{2}}\right) \nu(|\boldsymbol{x}-\boldsymbol{y}|) \mathrm{d} \boldsymbol{y}+F(u, f) .
$$

Such a minimization problem cannot be directly related to the nonlocal diffusion theory reported in this article because of the potential non-convexity of the functionals. For the same reasons, Gilboa and Osher (2007) introduced a modified convex functional (still based on nonlocal filters) that resembles common functionals used in PDE-based imaging approaches such as total variation functionals (Rudin, Osher and Fatemi 1992). In Section 21.1, we describe methods based on this concept and highlight their connection to the solution of nonlocal diffusion problems, including integral fractional problems. In all the examples below, unless otherwise stated, the relation between clean and noisy image is the additive noise model, i.e. $f=u+\eta$, where $u$ denotes the clean image, $f$ the noisy image and $\eta$ the noise.

\subsection{Image deblurring via minimization of a nonlocal functional}

Gilboa and Osher (2007) tackled the image deblurring problem by solving an unconstrained optimization problem with objective functional

$$
\mathcal{J}(u ; f)=\frac{1}{2}|u|_{V_{c}(\Omega)}^{2}+\frac{w}{2}\|u-f\|_{L^{2}(\Omega)}^{2},
$$

where $|\cdot|_{V_{c}}$ denotes the energy seminorm defined in (2.5) associated with an appropriate kernel $\gamma_{\delta}$, whose definition depends on the type of filtering (either NL-means or other neighbourhood filters) and the fidelity term controls the difference between the reconstructed image and the noisy image. Note that the weight $w \in \mathbb{R}^{+}$could be replaced by a spatially dependent function that is included within the $L^{2}$-norm.

The Euler-Lagrange equation that determines the necessary conditions for optimality is given by the nonlocal reaction-diffusion equation

$$
-\mathcal{L} u+w(u-f)=0
$$

whose well-posedness can be determined as described in Section 2.1 based on the properties of the kernel $\gamma_{\delta}$.

Figure 21.1, taken from Gilboa and Osher (2007), provides an example of a nonlocal deblurring reconstruction and also a comparison with a classical method local method, namely the total variation (TV) method of Rudin et al. (1992). The clean and noisy images are given in Figure 21.1(a, b) and the nonlocal and local reconstructions are given in Figure 21.1(c,d). These results show the superior ability of nonlocal filters to capture high-contrast features compared to classical methods.

Gilboa and Osher (2008), in a follow-up paper, provided additional insights about the theoretical aspects of the method of Gilboa and Osher 


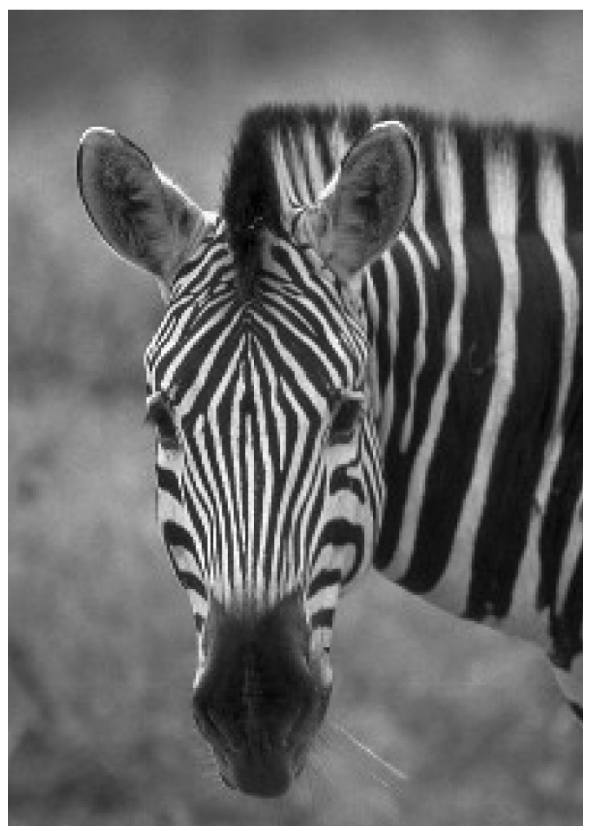

(a) clean

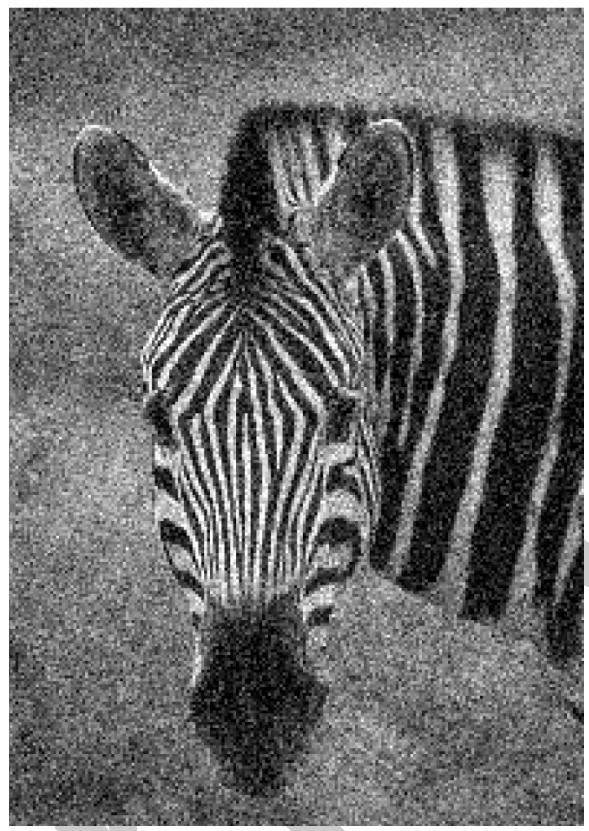

(b) noisy

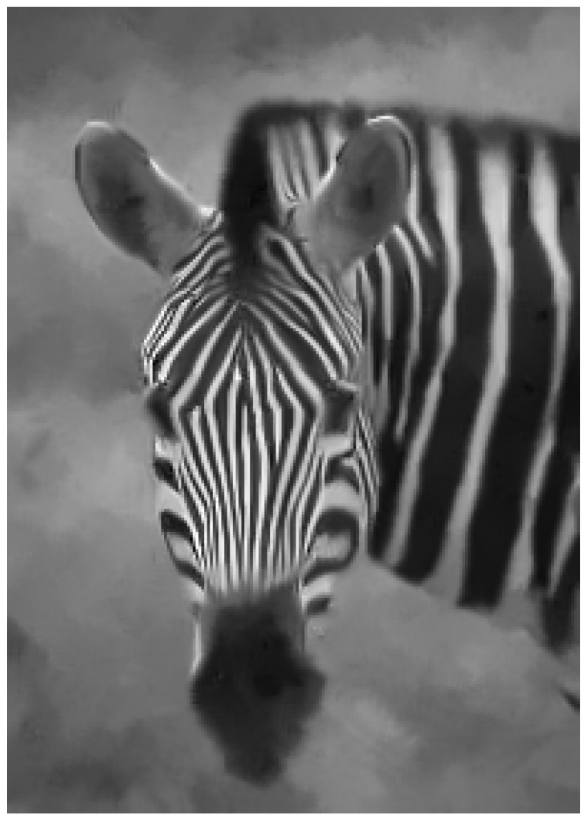

(c) nonlocal method

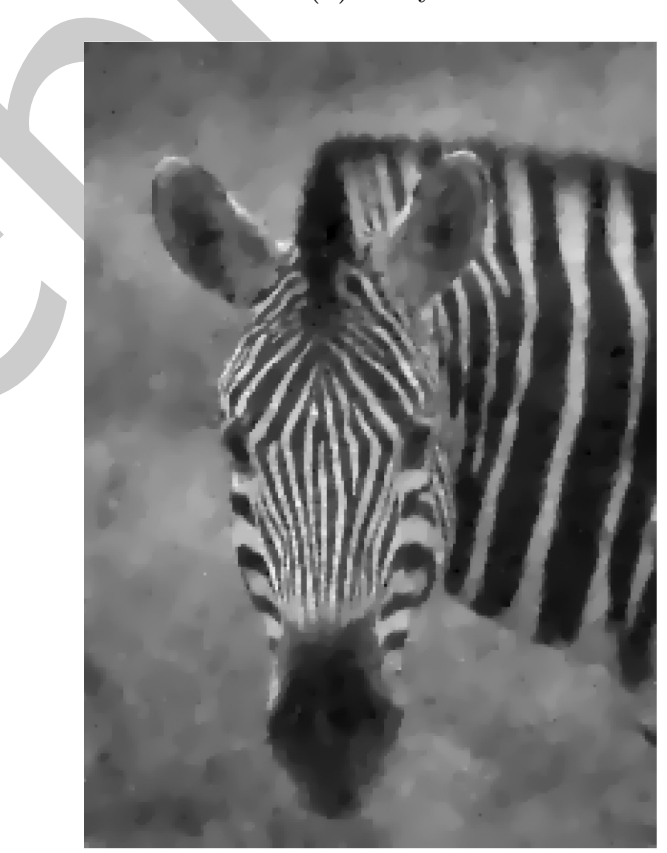

(d) local method (TV)

Figure 21.1. Clean, noisy, and nonlocal and local reconstructions of a noisy image featuring high-contrast features. 
(2007) and about other types of filters, and also provided comparisons with other methods.

Remark 21.1. A very similar formulation can be used for image deconvolution (Lou et al. 2010) where the relation between a clean and noisy image, $f=\mathcal{K} u+\eta$, involves a convolution operator $\mathcal{K}$. This renders the denoising problem more complicated. In this setting, the objective functional of the unconstrained optimization problem is given by

$$
\mathcal{J}_{C}(u)=\frac{1}{2}|u|_{V_{c}(\Omega)}^{2}+\frac{w}{2} \int_{\Omega}(k(\boldsymbol{x}) * u(\boldsymbol{x})-f(\boldsymbol{x}))^{2} \mathrm{~d} \boldsymbol{x}
$$

and the Euler-Lagrange equation is the nonlocal diffusion equation

$$
-\mathcal{L} u=w \widetilde{k} *(f-k * u)
$$

where $\tilde{k}$ denotes the adjoint of $k$.

Remark 21.2. Similar operators to those discussed in this section have also been used for image segmentation (Gilboa and Osher 2007), filtering (Darbon et al. 2008) and image and video recovery (Buades, Coll and Morel 2008).

Remark 21.3. In the literature, denoising nonlocal operators are not necessarily associated with nonlocal filters such as NL-means, but can be described by fractional operators, specifically by the spectral fractional Laplacian; see e.g. Antil and Bartels (2017).

\subsubsection{Optimization of the denoising parameters}

The quality of the reconstruction strongly depends on model parameters (e.g. parameters in the kernel) and on the weight parameter $w$. However, model parameters are often unknown (see Section 16) and the selection of $w$ is not a trivial task. D'Elia, De los Reyes and Trujillo (2019c) considered a bilevel optimization approach for the identification of kernel parameters (for integrable kernels, including NL-means) and of the weight function $w: \Omega \rightarrow \mathbb{R}^{+}$. Because the estimation of kernel parameters is discussed in Section 16, here we only consider the formulation for the identification of $w$, in its simplest setting.

Given a clean image and the corresponding blurred image, $(\hat{u}, \hat{f})$, the bilevel optimization problem is formulated as

$$
\min _{w \in W}\left(\mathcal{J}_{w}(u, w)=\|u-\hat{u}\|_{L^{2}(\Omega)}^{2}+\frac{\beta}{2}\|w\|_{H^{1}(\Omega)}^{2}\right)
$$

such that $u=\arg \min _{u \in V_{c}}\left(\mathcal{J}_{u}(u, w)=\frac{1}{2}|u|_{V_{c}(\Omega)}^{2}+\int_{\Omega} w(u-\hat{f})^{2} \mathrm{~d} \boldsymbol{x}\right)$, 
where the feasible set is $W=\left\{w \in H^{1}(\Omega): 0 \leq w(\boldsymbol{x}) \leq \mathrm{w}\right\}$, for some $\mathrm{w}<\infty$. Note that the constraint in (21.6) is equivalent to the diffusionreaction equation in (21.5). The well-posedness of the bilevel optimization problem has been proved by D'Elia et al. (2019c). They also introduce a second-order optimization algorithm for its solution, and give insights into implementation aspects and numerical performance. Also, they illustrate the theory and advantages of using nonlocal models via several numerical tests on standard benchmark images.

\section{Acknowledgements}

The research of MD, CG and (partially) MG is supported by Sandia National Laboratories (SNL). SNL is a multimission laboratory managed and operated by National Technology and Engineering Solutions of Sandia, LLC, a wholly owned subsidiary of Honeywell International, Inc., for the US Department of Energy's National Nuclear Security Administration under contract DE-NA-0003525. Specifically, this work was supported by the SNL Laboratory-directed Research and Development (LDRD) programme. Note that this paper describes objective technical results and analysis. Any subjective views or opinions that might be expressed in the paper do not necessarily represent the views of the US Department of Energy or the United States Government.

The research of QD is supported in part by the US National Foundation grants NSF DMS-1719699 and NSF CCF-1704833, the US Air Force Office of Scientific Research MURI Center for Material Failure Prediction Through Peridynamics, and by the US Army Research Office MURI grant W911NF15-1-0562.

The research of XT is supported in part by the US NSF grant DMS1819233.

The research of ZZ is supported by a start-up grant from the Hong Kong Polytechnic University and Hong Kong RGC grant 25300818.

Several figures in the article are reproduced by permission. Specifically, Figure 5.1 is reproduced from $\mathrm{Xu}$ et al. (2016a), Figures 12.1 and 12.2 from Tian and Engquist (2019), Figure 16.1 from D'Elia and Gunzburger (2014), Figure 16.2 from D'Elia and Gunzburger (2016), Figure 17.1 from Burkovska and Gunzburger (2019b), Figure 18.1 from Witman et al. (2017) and Figure 21.1 from Gilboa and Osher (2007). 
110 M. D’Elia, Q. Du, C. Glusa, M. Gunzburger, X. Tian and Z. Zhou

\section{REFERENCES ${ }^{8}$}

N. Abatangelo and L. Dupaigne (2017), 'Nonhomogeneous boundary conditions for the spectral fractional Laplacian', Ann. Inst. H. Poincaré Anal. Non Linéaire 34, 439-467.

M. Abramowitz and I. Stegun (1972), Handbook of Mathematical Functions with Formulas, Graphs, and Mathematical Tables, National Bureau of Standards and Dover.

G. Acosta and J.-P. Borthagaray (2017), 'A fractional Laplace equation: Regularity of solutions and finite element approximations', SIAM J. Numer. Anal. 55, 472-495.

G. Acosta, F. Bersetche and J. Borthagaray (2017), 'A short FE implementation for a 2D homogeneous Dirichlet problem of a fractional Laplacian', Comput. Math. Appl. 74, 784-816.

G. Acosta, J.-P. Borthagaray and N. Heuer (2019), 'Finite element approximations of the nonhomogeneous fractional Dirichlet problem', IMA J. Numer. Anal. 39, 1471-1501.

G. Acosta, J.-P. Borthagaray, O. Bruno and M. Maas (2018), 'Regularity theory and high order numerical methods for the (1D)-fractional Laplacian', Math. Comp. 87, 1821-1857.

M. Ainsworth and C. Glusa (2017), 'Aspects of an adaptive finite element method for the fractional Laplacian: a priori and a posteriori error estimates, efficient implementation and multigrid solver', Comput. Methods Appl. Mech. Engrg 327, $4-35$.

M. Ainsworth and C. Glusa (2018a), 'Hybrid finite element-spectral method for the fractional Laplacian approximation theory and efficient solver', SIAM J. Sci. Comput. 40, A2383-A2405.

M. Ainsworth and C. Glusa (2018b), Towards an efficient finite element method for the integral fractional Laplacian on polygonal domains. In Contemporary Computational Mathematics: A Celebration of the 80th Birthday of Ian Sloan (J. Dick et al., eds), Springer, pp. 17-57.

M. Ainsworth and Z. Mao (2017), 'Analysis and approximation of a fractional Cahn-Hilliard equation', SIAM J. Numer. Anal. 55, 1689-1718.

M. Ainsworth and W. McLean (2003), 'Multilevel diagonal scaling preconditioners for boundary element equations on locally refined meshes', Numer. Math. 93, $387-413$.

M. Ainsworth, W. McLean and T. Tran (1999), 'The conditioning of boundary element equations on locally refined meshes and preconditioning by diagonal scaling', SIAM J. Numer. Anal. 36, 1901-1932.

B. Aksoylu and T. Mengesha (2010), 'Results on nonlocal boundary value problems', Numer. Funct. Anal. Optim. 31, 1301-1317.

B. Aksoylu and M. Parks (2011), 'Variational theory and domain decomposition for nonlocal problems', Appl. Math. Comput. 217, 6498-6515.

8 The URLs cited in this work were correct at the time of going to press, but the publisher and the authors make no undertaking that the citations remain live or are accurate or appropriate. 
B. Aksoylu and Z. Unlu (2014), 'Conditioning analysis of nonlocal integral operators in fractional Sobolev spaces', SIAM J. Numer. Anal. 52, 653-677.

B. Alali, M. Gunzburger and K. Liu (2015), 'A generalized nonlocal calculus', J. Appl. Math. Phys. 223, 2807-2828.

F. Andreu, J. Mazón, J. Rossi and J. Toledo (2010), Nonlocal Diffusion Problems, Vol. 165 of Mathematical Surveys and Monographs, American Mathematical Society.

H. Antil and S. Bartels (2017), 'Spectral approximation of fractional PDEs in image processing and phase field modeling', Comput. Methods Appl. Math. 17, 661-678.

H. Antil and M. Warma (2020), 'Optimal control of fractional semilinear PDEs', ESAIM Control Optim. Calc. Var. 26, 5.

H. Antil, R. Khatri and M. Warma (2019a), 'External optimal control of nonlocal PDEs', Inverse Problems 35, 084003.

H. Antil, E. Otarola and A. J. Salgado (2016), 'A space-time fractional optimal control problem: Analysis and discretization', SIAM J. Control Optim. 54, $1295-1328$.

H. Antil, E. Otarola and A. J. Salgado (2018), 'Optimization with respect to order in a fractional diffusion model: Analysis, approximation and algorithmic aspects', J. Sci. Comput. 77, 204-224.

H. Antil, J. Pfefferer and S. Rogovs (2018), 'Fractional operators with inhomogeneous boundary conditions: Analysis, control, and discretization', Commun. Math. Sci. 16, 1395-1426.

H. Antil, D. Verma and M. Warma (2019b), External optimal control of fractional parabolic PDEs. arXiv:1904.07123

K. Atkinson and W. Han (2012), Spherical Harmonics and Approximations on the Unit Sphere: An Introduction, Vol. 2044 of Lecture Notes in Mathematics, Springer.

I. Babuška (1971), 'Error-bounds for finite element method', Numer. Math. 16, $322-333$.

I. Babuška and K. Aziz (1972), Survey lectures on the mathematical foundation of the finite element method. In The Mathematical Foundations of the Finite Element Method with Application to Partial Differential Equations, Academic Press, pp. 1-359.

B. Baeumer and M. Meerschaert (2010), 'Tempered stable Lévy motion and transient super-diffusion', J. Comput. Appl. Math. 233, 2438-2448.

D. Baleanu, K. Diethelm, E. Scalas and J. Trujillo (2016), Fractional Calculus: Models and Numerical Methods, World Scientific.

L. Banjai, J. Melenk, R. Nochetto, E. Otárola, A. Salgado and C. Schwab (2019), 'Tensor FEM for spectral fractional diffusion', Found. Comput. Math. 19, 901-962.

U. Biccari and V. Hernández-Santamaría (2018), 'The Poisson equation from nonlocal to local', Electron. J. Differential Equations 2018, 145.

R. Blumenthal, R. Getoor and D. Ray (1961), 'On the distribution of first hits for the symmetric stable processes', Trans. Amer. Math. Soc. 99, 540-554.

F. Bobaru and M. Duangpanya (2010), 'The peridynamic formulation for transient heat conduction', Int. J. Heat Mass Transfer 53, 4047-4059. 
F. Bobaru and G. Zhang (2015), 'Why do cracks branch? A peridynamic investigation of dynamic brittle fracture', Int. J. Fracture 196, 59-98.

F. Bobaru, M. Yang, L. Alves, S. Silling, E. Askari and J. Xu (2009), 'Convergence, adaptive refinement, and scaling in 1D peridynamics', Inter. J. Numer. Meth. in Eng. 77, 852-877.

K. Bogdan and T. Byczkowski (1999), 'Potential theory for the $\alpha$-stable Schrödinger operator on bounded Lipschitz domains', Studia Math. 133, 53-92.

K. Bogdan, K. Burdzy and Z.-Q. Chen (2003), 'Censored stable processes', Probab. Theory Related Fields 127, 89-152.

A. Bonito and J. Pasciak (2015), 'Numerical approximation of fractional powers of elliptic operators', Math. Comp. 84, 2083-2110.

A. Bonito and J. Pasciak (2017), 'Numerical approximation of fractional powers of regularly accretive operators', IMA J. Numer. Anal. 37, 1245-1273.

A. Bonito, W. Lei and J. Pasciak (2019), 'Numerical approximation of the integral fractional Laplacian', Numer. Math. 142, 235-278.

A. Bonito, W. Lei and A. Salgado (2020), 'Finite element approximation of an obstacle problem for a class of integro-differential operators', ESAIM Math. Model. Numer. Anal. 54, 229-253.

J.-P. Borthagaray, R. Nochetto and A. Salgado (2019), 'Weighted Sobolev regularity and rate of approximation of the obstacle problem for the integral fractional Laplacian', Math. Model. Meth. Appl. Sci. 29, 2670-2717.

J. Bourgain, H. Brezis and P. Mironescu (2001), Another Look at Sobolev Spaces, IOS Press.

M. Bourgoin, N. Ouellette, H. Xu, J. Berg and E. Bodenschatz (2006), 'The role of pair dispersion in turbulent flow', Science 311, 835-838.

J. Boyd (1987), 'Spectral methods using rational basis functions on an infinite interval', J. Comput. Phys. 69, 112-142.

J. Boyd (2001), 'Rational Chebyshev spectral methods for unbounded solutions on an infinite interval using polynomial-growth special basis functions', Comput. Math. Appl. 41, 1293-1315.

S. Brenner and R. Scott (1994), The Mathematical Theory of Finite Element Methods, Springer.

A. Buades, B. Coll and J. Morel (2008), 'Nonlocal image and movie denoising', Int. J. Comput. Vision 76, 123-139.

A. Buades, B. Coll and J. Morel (2010), 'Image denoising methods: A new nonlocal principle', SIAM Rev. 52, 113-147.

C. Bucur (2016), 'Some observations on the Green function for the ball in the fractional Laplace framework', Commun. Pure Appl. Anal. 15, 657-699.

M. Buhmann (2000), Radial basis functions. In Acta Numerica, Vol. 9, Cambridge University Press, pp. 1-38.

M. Buhmann (2003), Radial Basis Functions: Theory and Implementations, Vol. 12 of Cambridge Monographs on Applied and Computational Mathematics, Cambridge University Press.

N. Burch and R. Lehoucq (2015), 'Computing the exit-time for a finite-range symmetric jump process', Monte Carlo Methods Appl. 21, 139-152.

N. Burch, M. D'Elia and R. Lehoucq (2014), 'The exit-time problem for a Markov jump process', Eur. Phys. J. Special Topics 223, 3257-3271. 
O. Burkovska and M. Gunzburger (2019a), Affine approximation of parametrized kernels and model order reduction for nonlocal and fractional Laplace models. arXiv: 1901.0674

O. Burkovska and M. Gunzburger (2019b), 'Regularity and approximation analyses of nonlocal variational equality and inequality problems', J. Math. Anal. Appl. 478, 1027-1048.

L. Caffarelli and L. Silvestre (2007), 'An extension problem related to the fractional Laplacian', Comm. Partial Differential Equations 32, 1245-1260.

L. Caffarelli and P. Stinga (2016), 'Fractional elliptic equations, Caccioppoli estimates and regularity', Ann. Inst. H. Poincaré Anal. Non Linéaire 33, 767-807.

L. Caffarelli, X. Ros-Oton and J. Serra (2017), 'Obstacle problems for integrodifferential operators: Regularity of solutions and free boundaries', Invent. Math. 208, 1155-1211.

C. Çelik and M. Duman (2012), 'Crank-Nicolson method for the fractional diffusion equation with the Riesz fractional derivative', J. Comput. Phys. 231, 17431750 .

A. Chen, Q. Du, C. Li and Z. Zhou (2017), 'Asymptotically compatible schemes for space-time nonlocal diffusion equations', Chaos Solitons Fractals 102, 361371.

L. Chen, R. Nochetto, E. Otárola and A. J. Salgado (2016a), 'Multilevel methods for nonuniformly elliptic operators and fractional diffusion', Math. Comp. 85, 2583-2607.

M. Chen and W. Deng (2014), 'Fourth order accurate scheme for the space fractional diffusion equations', SIAM J. Numer. Anal. 52, 1418-1438.

S. Chen, J. Shen and L.-L. Wang (2016b), 'Generalized Jacobi functions and their applications to fractional differential equations', Math. Comp. 85, 1603-1638.

W. Chen (2006), 'A speculative study of 2/3-order fractional Laplacian modeling of turbulence: Some thoughts and conjectures', Chaos 16, 023126.

X. Chen and M. Gunzburger (2011), 'Continuous and discontinuous finite element methods for a peridynamics model of mechanics', Comput. Methods Appl. Mech. Engrg 200, 1237-1250.

Z.-Q. Chen and P. Kim (2002), 'Green function estimate for censored stable processes', Probab. Theory Related Fields 124, 595-610.

A. Chernov, T. von Petersdorff and C. Schwab (2011), 'Exponential convergence of $h p$ quadrature for integral operators with Gevrey kernels', ESAIM Math. Model. Numer. Anal. 45, 387-422.

P. Ciarlet (2002), Finite Element Methods for Elliptic Problems, SIAM.

C. Cortazar, M. Elgueta, J. Rossi and N. Wolanski (2008), 'How to approximate the heat equation with Neumann boundary conditions by nonlocal diffusion problems', Arch. Rat. Mech. Anal. 187, 137-156.

T. Coulhon and A. Grigoryan (1998), 'Random walks on graphs with regular volume growth', Geom. Funct. Anal. 8, 656-701.

J. Curtiss (1950), Sampling methods applied to differential and difference equations. In Proceedings, Seminar on Scientific Computation, November 1949, IBM, pp. 87-109.

N. Cusimano, F. del Teso, L. Gerardo-Giorda and G. Pagnini (2018), 'Discretizations of the spectral fractional Laplacian on general domains with Dirichlet, 
114 M. D’Elia, Q. Du, C. Glusa, M. Gunzburger, X. Tian and Z. Zhou

Neumann, and Robin boundary conditions', SIAM J. Numer. Anal. 56, 12431272 .

J. Darbon, A. Cunha, T. Chan, S. Osher and G. Jensen (2008), Fast nonlocal filtering applied to electron cryomicroscopy. In 2008 5th IEEE International Symposium on Biomedical Imaging: From Nano to Macro, IEEE, pp. 13311334 .

F. Del Teso, J. Endal and E. Jakobsen (2018), 'Robust numerical methods for nonlocal (and local) equations of porous medium type, II: Schemes and experiments', SIAM J. Numer. Anal. 56, 3611-3647.

F. Del Teso, J. Endal and E. Jakobsen (2019), 'Robust numerical methods for nonlocal (and local) equations of porous medium type, I: Theory', SIAM J. Numer. Anal. 57, 2266-2299.

M. D'Elia and M. Gunzburger (2013), 'The fractional Laplacian operator on bounded domains as a special case of the nonlocal diffusion operator', Comput. Math. Appl. 66, 1245-1260.

M. D'Elia and M. Gunzburger (2014), 'Optimal distributed control of nonlocal steady diffusion problems', SIAM J. Control Optim. 55, 667-696.

M. D'Elia and M. Gunzburger (2016), 'Identification of the diffusion parameter in nonlocal steady diffusion problems', Appl. Math. Optim. 73, 227-249.

M. D'Elia, J.-C. De los Reyes and A. M. Trujillo (2019c), Bilevel parameter optimization for nonlocal image denoising models. arXiv:1912.02347

M. D'Elia, Q. Du, M. Gunzburger and R. B. Lehoucq (2017), 'Nonlocal convectiondiffusion problems on bounded domains and finite-range jump processes', Comput. Methods Appl. Math. 17, 707-722.

M. D'Elia, C. Flores, X. Li, P. Radu and Y. Yu (2019a), Helmholtz-Hodge decompositions in the nonlocal framework: Well-posedness analysis and applications. arXiv: 1906.04259

M. D'Elia, C. Glusa and E. Otárola (2019b), 'A priori error estimates for the optimal control of the integral fractional Laplacian', SIAM J. Control Optim. 57, 2775-2798.

M. D'Elia, X. Tian and Y. Yu $(2019 d)$, A physically-consistent, flexible and efficient strategy to convert local boundary conditions into nonlocal volume constraints. arXiv:1906:04259

B. Deng, Z. Zhang and X. Zhao (2019), 'Superconvergence points for the spectral interpolation of Riesz fractional derivatives', J. Sci. Comput. 81, 1577-1601.

W. Deng, B. Li, W. Tian, and P. Zhang, (2018), 'Boundary problems for the fractional and tempered fractional operators', Multiscale Model. Simul. 16, 125-149.

E. Di Nezza, G. Palatucci and E. Valdinoci (2012), 'Hitchhiker's guide to the fractional Sobolev spaces', Bull. Sci. Math. 136, 521-573.

H. Ding, C. Li and Y.-Q. Chen (2015), 'High-order algorithms for Riesz derivative and their applications, II', J. Comput. Phys. 293, 218-237.

S. Dipierro, X. Ros-Oton and E. Valdinoci (2017), 'Nonlocal problems with Neumann boundary conditions', Rev. Mat. Iberoamer. 33, 377-416.

Q. Du (2019), Nonlocal Modeling, Analysis, and Computation, Vol. 94 of CBMSNSF Conference Series in Applied Mathematics, SIAM. 
Q. Du and X. Feng (2020), The phase field method for geometric moving interfaces and their numerical approximations. In Geometric Partial Differential Equations, Part I, Vol. 21 of Handbook of Numerical Analysis, Elsevier.

Q. Du and Z. Huang (2017), 'Numerical solution of a scalar one-dimensional monotonicity-preserving nonlocal conservation law', J. Math. Res. Appl. 37, $1-18$.

Q. Du and X. Tian (2014), Asymptotically compatible schemes for peridynamics based on numerical quadratures. In ASME 2014 International Mechanical Engineering Congress and Exposition, IMECE2014-39620.

Q. Du and X. Tian (2015), Robust discretization of nonlocal models related to peridynamics. In Meshfree Methods for Partial Differential Equations VII, Vol. 100 of Lecture Notes in Computational Science and Engineering, Springer, pp. $97-113$.

Q. Du and X. Tian (2018), Heterogeneously localized nonlocal operators, boundary traces and variational problems. In Proceedings of the International Congress of Chinese Mathematicians, Vol. 43 of the ALM Series, International Press, pp. 217-236.

Q. Du and X. Tian (2019), 'Mathematics of smoothed particle hydrodynamics via a nonlocal Stokes equation', Found. Comput. Math. doi:10.1007/s10208-01909432-0

Q. Du and J. Yang (2016), 'Asymptotically compatible Fourier spectral approximations of nonlocal Allen-Cahn equations', SIAM J. Numer. Anal. 54, 18991919.

Q. Du and J. Yang (2017), 'Fast and accurate implementation of Fourier spectral approximations of nonlocal diffusion operators and its applications', J. Comput. Phys. 332, 118-134.

Q. Du and X. Yin (2019), 'A conforming DG method for linear nonlocal models with integrable kernels', J. Sci. Comput. 80, 1913-1935.

Q. Du and K. Zhou (2011), 'Mathematical analysis for the peridynamic nonlocal continuum theory', ESAIM Math. Model. Numer. Anal. 45, 217-234.

Q. Du and Z. Zhou (2017), Multigrid finite element method for nonlocal diffusion equations with a fractional kernel. Preprint.

Q. Du, M. Gunzburger, R. Lehoucq and K. Zhou (2012a), 'Analysis and approximation of nonlocal diffusion problems with volume constraints', SIAM Rev. 54, 667-696.

Q. Du, M. Gunzburger, R. Lehoucq and K. Zhou (2013a), 'A nonlocal vector calculus, nonlocal volume-constrained problems, and nonlocal balance laws', Math. Model. Meth. Appl. Sci. 23, 493-540.

Q. Du, H. Han, C. Zheng and J. Zhang (2018a), 'Numerical solution of a twodimensional nonlocal wave equation on unbounded domains', SIAM J. Sci. Comput. 40, A1430-1445.

Q. Du, Z. Huang and P. LeFloch (2017a), 'Nonlocal conservation laws, I: A new class of monotonicity-preserving models', SIAM J. Numer. Anal. 55, 24652489.

Q. Du, Z. Huang and R. Lehoucq (2014), 'Nonlocal convection-diffusion volumeconstrained problems and jump processes', Discrete Contin. Dyn. Syst. B 19, 961-977. 
Q. Du, L. Ju and J. Lu (2019a), 'Analysis of fully discrete approximations for dissipative systems and application to time-dependent nonlocal diffusion problems', J. Sci. Comput. 78, 1438-1466.

Q. Du, L. Ju and J. Lu (2019b), 'A discontinuous Galerkin method for one-dimensional time-dependent nonlocal diffusion problems', Math. Comp. 88, 123147.

Q. Du, L. Ju, X. Li and Z. Qiao (2018b), 'Stabilized linear semi-implicit schemes for the nonlocal Cahn-Hilliard equation', J. Comput. Phys. 363, 39-54.

Q. Du, L. Ju, J. Lu and X. Tian (2020a), 'A discontinuous Galerkin method with penalty for one-dimensional nonlocal diffusion problems', Comm. Appl. Math. Comput. 2, 31-55.

Q. Du, L. Ju, L. Tian and K. Zhou (2013b), 'A posteriori error analysis of finite element method for linear nonlocal diffusion and peridynamic models', Math. Comp. 82, 1889-1922.

Q. Du, J. Kamm, R. Lehoucq and M. Parks (2012b), 'A new approach for a nonlocal, nonlinear conservation law', SIAM J. Appl. Math. 72, 464-487.

Q. Du, R. Lipton and T. Mengesha (2016a), 'Multiscale analysis of linear evolution equations with applications to nonlocal models for heterogeneous media', ESAIM Math. Model. Numer. Anal. 50, 1425-1455.

Q. Du, Y. Tao and X. Tian (2018c), 'A peridynamic model of fracture mechanics with bond-breaking', J. Elasticity 132, 197-218.

Q. Du, Y. Tao, X. Tian and J. Yang (2016b), 'Robust a posteriori stress analysis for quadrature collocation approximations of nonlocal models via nonlocal gradients', Comput. Methods Appl. Mech. Engrg 310, 605-627.

Q. Du, Y. Tao, X. Tian and J. Yang (2019c), 'Asymptotically compatible discretization of multidimensional nonlocal diffusion models and approximations of nonlocal Green's functions', IMA J. Numer. Anal. 39, 607-625.

Q. Du, L. Tian and X. Zhao (2013c), 'A convergent adaptive finite element algorithm for nonlocal diffusion and peridynamic models', SIAM J. Numer. Anal. 51, 1211-1234.

Q. Du, L. Toniazzi and Z. Zhou (2020b), 'Stochastic representation of solution to nonlocal-in-time diffusion', Stoch. Proc. Appl. 10, 2058-2085.

Q. Du, J. Yang and Z. Zhou (2017b), 'Analysis of a nonlocal-in-time parabolic equation', Discrete Contin. Dyn. Syst. B 22, 339-368.

Q. Du, J. Zhang and C. Zheng (2018d), 'Nonlocal wave propagation in unbounded multi-scale media', Commun. Comput. Phys. 24, 1049-1072.

S. Duo and Y. Zhang (2019), 'Accurate numerical methods for two and three dimensional integral fractional Laplacian with applications', Comput. Methods Appl. Mech. Engrg 355, 639-662.

S. Duo, H.-W. van Wyk and Y. Zhang (2018), 'A novel and accurate finite difference method for the fractional Laplacian and the fractional Poisson problem', J. Comput. Phys. 355, 233-252.

B. Dyda, A. Kuznetsov and M. Kwaśnicki (2017a), 'Eigenvalues of the fractional Laplace operator in the unit ball', J. Lond. Math. Soc. (2) 95, 500-518.

B. Dyda, A. Kuznetsov and M. Kwaśnicki (2017b), 'Fractional Laplace operator and Meijer G-function', Constr. Approx. 45, 427-448. 
A. Ern and J.-L. Guermond (2004), Theory and Practice of Finite Elements, Springer.

V. Ervin and J. Roop (2007), 'Variational solution of fractional advection dispersion equations on bounded domains in $\mathbb{R}^{d}$, Numer. Methods Partial Differential Equations 23, 256-281.

V. Ervin, N. Heuer and J. Roop (2018), 'Regularity of the solution to 1-D fractional order diffusion equations', Math. Comp. 87, 2273-2294.

L. Evans (1998), Partial Differential Equations, American Mathematical Society.

M. Faustmann, J. Melenk, M. Parvizi and D. Praetorius (2019), Optimal adaptivity and preconditioning for the fractional Laplacian. In Proceedings of WONAPDE.

M. Felsinger, M. Kassmann and P. Voigt (2015), 'The Dirichlet problem for nonlocal operators', Math. Z. 279, 779-809.

A. Fuensanta and J. Muñoz (2015), 'Nonlocal optimal design: A new perspective about the approximation of solutions in optimal design', J. Math. Anal. Appl. 429, 288-310.

C. Gal and M. Warma (2016), 'Elliptic and parabolic equations with fractional diffusion and dynamic boundary conditions', Evol. Equ. Control Theory 5, 61-103.

G. Gilboa and S. Osher (2007), 'Nonlocal linear image regularization and supervised segmentation', Multiscale Model. Simul. 6, 595-630.

G. Gilboa and S. Osher (2008), 'Nonlocal operators with applications to image processing', Multiscale Model. Simul. 7, 1005-1028.

V. Girault and P.-A. Raviart (1986), Finite Element Methods for Navier-Stokes Equations, Springer.

C. Glusa and E. Otárola (2019), Optimal control of a parabolic fractional PDE: Analysis and discretization. arXiv:1905.10002

I. Gradshteyn and I. Ryzhik (2007), Table of Integrals, Series, and Products, Elsevier/Academic Press.

G. Grubb (2015a), 'Fractional Laplacians on domains, a development of Hörmander's theory of $\mu$-transmission pseudodifferential operators', Adv. Math. 268, 478-528.

G. Grubb (2015b), 'Spectral results for mixed problems and fractional elliptic operators', J. Math. Anal. Appl. 421, 1616-1634.

G. Grubb (2016), 'Regularity of spectral fractional Dirichlet and Neumann problems', Math. Nachrichten 289, 831-844.

Q. Guan and M. Gunzburger (2015), 'Stability and accuracy of time-stepping schemes and dispersion relations for a nonlocal wave equation', Numer. Method. Partial Differential Equations 31, 500-516.

Q. Guan and M. Gunzburger (2017), 'Analysis and approximation of a nonlocal obstacle problem', J. Comput. Appl. Math. 313, 102-118.

Q. Guan, M. Gunzburger, C. Webster and G. Zhang (2017), 'Reduced basis methods for nonlocal diffusion problems with random input data', Comput. Methods Appl. Mech. Engrg 317, 746-770.

M. Gunzburger and R. Lehoucq (2010), 'A nonlocal vector calculus with application to nonlocal boundary value problems', Multiscale Model. Simul. 8, 1581-1598. 
M. Gunzburger, N. Jiang and F. Xu (2018), 'Analysis and approximation of a fractional Laplacian-based closure model for turbulent flows and its connection to Richardson pair dispersion', Comput. Math. Appl. 75, 1973-2001.

B. Guo and L. Wang (2004), 'Jacobi approximations in non-uniformly Jacobiweighted Sobolev spaces', J. Approx. Theory 128, 1-41.

W. Hackbusch (1985), Multi-Grid Methods and Applications, Springer.

W. Hackbusch (1994), Iterative Solution of Large Sparse Systems of Equations, Springer.

W. Hu, Y. Ha and F. Bobaru (2012), 'Peridynamic model for dynamic fracture in unidirectional fiber-reinforced composites', Comput. Methods Appl. Mech. Engrg 217, 247-261.

Y. Huang and A. Oberman (2014), 'Numerical methods for the fractional Laplacian: A finite difference-quadrature approach', SIAM J. Numer. Anal. 52, 3056-3084.

P. Jha and R. Lipton (2019), 'Numerical convergence of finite difference approximations for state based peridynamic fracture models', Comput. Methods Appl. Mech. Engrg 351, 184-225.

P. Jha and R. Lipton (2020), 'Finite element convergence for state-based peridynamic fracture models', Comm. Appl. Math. Comput. 2, 93-128.

X. Jiang, L.-H. Lim, Y. Yao and Y. Ye (2011), 'Statistical ranking and combinatorial Hodge theory', Math. Prog. 127, 203-244.

M. Kac (1949), 'On distributions of certain Wiener functionals', Trans. Amer. Math. Soc. 65, 1-13.

G. Karniadakis, ed. (2019), Handbook of Fractional Calculus with Applications, Vol. 3, Numerical Methods, De Gruyter.

S. Kindermann, S. Osher and P. Jones (2005), 'Deblurring and denoising of images by nonlocal functionals', SIAM Multiscale Model. Simul. 4, 1091-1115.

A. Kyprianou, A. Osojnik and T. Shardlow (2018), 'Unbiased 'walk-on-spheres' Monte Carlo methods for the fractional Laplacian', IMA J. Numer. Anal. 38, $1550-1578$.

H. Lee and Q. Du (2019), 'Asymptotically compatible SPH-like particle discretizations of one dimensional linear advection models', SIAM J. Numer. Anal. 57, $127-147$.

H. Lee and Q. Du (2020), 'Nonlocal gradient operators with a nonspherical interaction neighborhood and their applications', ESAIM Math. Model. Numer. Anal. 54, 105-128.

R. Lehoucq and S. Rowe (2016), 'A radial basis function Galerkin method for inhomogeneous nonlocal diffusion', Comput. Methods Appl. Mech. Engrg 299, 366-380.

R. Lehoucq, F. Narcowich, S. Rowe and J. Ward (2018), 'A meshless Galerkin method for non-local diffusion using localized kernel bases', Math. Comp. 87, 2233-2258.

S.-L. Lei and H.-W. Sun (2013), 'A circulant preconditioner for fractional diffusion equations', J. Comput. Phys. 242, 715-725.

Y. Leng, X. Tian, N. Trask and J. Foster (2019), Asymptotically compatible reproducing kernel collocation and meshfree integration for nonlocal diffusion. arXiv:1907.12031 
Y. Leng, X. Tian, N. Trask and J. Foster (2020), Asymptotically compatible reproducing kernel collocation and meshfree integration for peridynamic Navier equation. arXiv:2001.00649

O. Lézoray, V.-T. Ta and A. Elmoataz (2010), 'Partial differences as tools for filtering data on graphs', Pattern Recog. Lett. 31, 2201-2213.

C. Li and A. Chen (2018), 'Numerical methods for fractional partial differential equations', Int. J. Comput. Math. 95, 1048-1099.

S. Li and W. Liu (1996), 'Moving least-square reproducing kernel method, II: Fourier analysis', Comput. Methods Appl. Mech. Engrg 139, 159-193.

X. Li, Z. Qiao and C. Wang (2019), Convergence analysis for a stabilized linear semi-implicit numerical scheme for the nonlocal Cahn-Hilliard equation. arXiv:1902.04967

R. Lipton (2014), 'Dynamic brittle fracture as a small horizon limit of peridynamics', J. Elasticity 117, 21-50.

R. Lipton (2017), 'Cohesive dynamics and brittle fracture', J. Elasticity 124, 143191.

R. Lipton, R. Lehoucq and K. Sha (2018), 'Complex fracture nucleation and evolution with nonlocal elastodynamics', J. Peridynamics Nonlocal Model. 1, 122130.

W. Liu, S. Jun and Y. Zhang (1995), 'Reproducing kernel particle methods', Int. J. Numer. Methods Fluids 20, 1081-1106.

Y. Lou, X. Zhang, S. Osher and A. Bertozzi (2010), 'Image recovery via nonlocal operators', J. Sci. Comput. 42, 185-197.

F. Mainardi (1997), Fractional calculus. In Fractals and Fractional Calculus in Continuum Mechanics, Vol. 378 of International Centre for Mechanical Sciences (Courses and Lectures), Springer, pp. 291-348.

Z. Mao and G. Karniadakis (2018), A spectral method (of exponential convergence) for singular solutions of the diffusion equation with general two-sided fractional derivative', SIAM J. Numer. Anal. 56, 24-49.

Z. Mao and J. Shen (2017), 'Hermite spectral methods for fractional PDEs in unbounded domains', SIAM J. Sci. Comput. 39, A1928-A1950.

Z. Mao and J. Shen (2018), 'Spectral element method with geometric mesh for two-sided fractional differential equations', Adv. Comput. Math. 44, 745-771.

Z. Mao, S. Chen and J. Shen (2016), 'Efficient and accurate spectral method using generalized Jacobi functions for solving Riesz fractional differential equations', Appl. Numer. Math. 106, 165-181.

W. McLean (2000), Strongly Elliptic Systems and Boundary Integral Equations, Cambridge University Press.

M. Meerschaert and A. Sikorskii (2012), Stochastic Models for Fractional Calculus, De Gruyter.

M. Meerschaert and C. Tadjeran (2004), 'Finite difference approximations for fractional advection-dispersion flow equations', J. Comput. Appl. Math. 172, 65-77.

M. Meerschaert and C. Tadjeran (2006), 'Finite difference approximations for twosided space-fractional partial differential equations', Appl. Numer. Math. 56, 80-90. 
M. Meerschaert, D. Benson and B. Bäumer (1999), 'Multidimensional advection and fractional dispersion', Phys. Rev. E 59, 5026-5028.

D. Meidner, J. Pfefferer, K. Schürholz and B. Vexler (2018), ' $h p$-finite elements for fractional diffusion', SIAM J. Numer. Anal. 56, 2345-2374.

T. Mengesha and Q. Du (2013), 'Analysis of a scalar nonlocal peridynamic model with sign changing kernel', Discrete Contin. Dyn. Syst. B 18, 1415-1437.

T. Mengesha and Q. Du (2014), 'Nonlocal constrained value problems for a linear peridynamic Navier equation', J. Elasticity 116, 27-51.

T. Mengesha and Q. Du (2015), 'On the variational limit of a class of nonlocal functionals related to peridynamics', Nonlinearity 28, 3999-4035.

T. Mengesha and Q. Du (2016), 'Characterization of function spaces of vector fields and an application in nonlinear peridynamics', Nonlin. Anal. Theory Methods Appl. 140, 82-111.

R. Metzler and J. Klafter (2000), 'The random walk's guide to anomalous diffusion: A fractional dynamics approach', Phys. Rep. 339, 77.

V. Minden and L. Ying (2018), A simple solver for the fractional Laplacian in multiple dimensions. arXiv:1802.03770

S. Molčanov and E. Ostrovskiı̌ (1969), 'Symmetric stable processes as traces of degenerate diffusion processes', Teor. Verojatnost. i Primenen. 14, 127-130.

M. Muller (1956), 'Some continuous Monte Carlo methods for the Dirichlet problem', Ann. Math. Statist. 27, 569-589.

J. Necas (1967), Les Méthodes Directes en Théorie des Équations Elliptiques, Masson.

J. Necas (2016), 'Sur une méthode pour résoudre les équations aux dérivées partielles de type elliptique, voisine de la variationnelle', Ann. Scuola Norm. Sup. Pisa 50, 1425-1455.

R. Nochetto, E. Otárola and A. Salgado (2015), 'A PDE approach to fractional diffusion in general domains: a priori error analysis', Found. Comput. Math. 15, $733-791$.

R. Nochetto, T. von Petersdorff and C.-S. Zhang (2010), 'A posteriori error analysis for a class of integral equations and variational inequalities', Numer. Math. 116, 519-552.

M. Ortigueira (2006), 'Riesz potential operators and inverses via fractional centred derivatives', Int. J. Math. Math. Sci. 2006, 48391.

E. Otárola and A. Salgado (2018), 'Sparse optimal control for fractional diffusion', Comput. Methods Appl. Math. 18, 95-110.

J. Pan, M. Ng and H. Wang (2016), 'Fast iterative solvers for linear systems arising from time-dependent space-fractional diffusion equations', SIAM J. Sci. Comput. 38, A2806-A2826.

G. Pang, W. Chen and Z. Fu (2015), 'Space-fractional advection-dispersion equations by the Kansa method', J. Comput. Phys. 293, 280-296.

G. Pang, M. D'Elia, M. Parks and G. Karniadakis (2019a), nPINNs: Nonlocal physics-informed neural networks. Manuscript.

G. Pang, L. Lu and G. Karniadakis (2019b), 'fPINNs: Fractional physics-informed neural networks', SIAM J. Sci. Comput. 41, A2603-A2626. 
I. Podlubny (1999), Fractional Differential Equations: An Introduction to Fractional Derivatives, Fractional Differential Equations, to Methods of their Solution and Some of their Applications, Vol. 198 of Mathematics in Science and Engineering, Academic Press.

L. Richardson (1926), 'Atmospheric diffusion shown on a distance-neighbour graph', Proc. Roy. Soc. London A 110, 023126.

X. Ros-Oton (2016), 'Nonlocal elliptic equations in bounded domains: A survey', Publ. Mat. 60, 3-26.

X. Ros-Oton and J. Serra (2014), 'The Dirichlet problem for the fractional Laplacian: Regularity up to the boundary', J. Math. Pures Appl. 101, 275-302.

J. Rosenfeld, S. Rosenfeld and W. E. Dixon (2019), 'A mesh-free pseudospectral approach to estimating the fractional Laplacian via radial basis functions', J. Comput. Phys. 390, 306-322.

L. Rudin, S. Osher and E. Fatemi (1992), 'Nonlinear total variation based noise removal algorithms', Phys. D Nonlin. Phenomena 60, 259-268.

K. Sabelfeld and N. Simonov (1994), Random Walks on Boundary for Solving $P D E s, \mathrm{VSP}$.

J. Salazar and L. Collins (2009), 'Two-particle dispersion in isotropic turbulent flows', Ann. Rev. Fluid Mech. 41, 405-432.

M. Samiee, M. Zayernouri and M. Meerschaert (2019a), 'A unified spectral method for FPDEs with two-sided derivatives, I: A fast solver', J. Comput. Phys. 385, $225-243$.

M. Samiee, M. Zayernouri and M. Meerschaert (2019b), 'A unified spectral method for FPDEs with two-sided derivatives, II: Stability, and error analysis', J. Comput. Phys. 385, 244-261.

S. Sauter and C. Schwab (2010), Boundary element methods. In Boundary Element Methods, Vol. 39 of Springer Series in Computational Mathematics, Springer, pp. $183-287$.

P. Seleson, Q. Du and M. Parks (2016), 'On the consistency between nearestneighbor peridynamic discretizations and discretized classical elasticity models', Comput. Methods Appl. Mech. Engrg 311, 698-722.

P. Seleson, M. Parks, M. Gunzburger and R. Lehoucq (2009), 'Peridynamics as an upscaling of molecular dynamics', Multiscale Model. Simul. 8, 204-227.

R. Servadei and E. Valdinoci (2013), 'Lewy-Stampacchia type estimates for variational inequalities driven by (non)local operators', Rev. Mat. Iberoamer. 29, 1091-1126.

R. Servadei and E. Valdinoci (2014), 'On the spectrum of two different fractional operators', Proc. Roy. Soc. Edinburgh Sect. A 144, 831-855.

T. Shardlow (2019), 'A walk outside spheres for the fractional Laplacian: Fields and first eigenvalue', Math. Comp. 88, 2767-2792.

J. Shen and L.-L. Wang (2009), 'Some recent advances on spectral methods for unbounded domains', Commun. Comput. Phys. 5, 195-241.

J. Shen, T. Tang and L.-L. Wang (2011), Spectral Methods, Vol. 41 of Springer Series in Computational Mathematics, Springer.

C. Sheng, J. Shen, T. Tang, L.-L. Wang and H. Yuan (2019), Fast Fourier-like mapped Chebyshev spectral-Galerkin methods for PDEs with integral fractional Laplacian in unbounded domains. arXiv:1908.10029 
S. Silling (2000), 'Reformulation of elasticity theory for discontinuities and longrange forces', J. Mech. Phys. Solids 48, 175-209.

S. Silling (2010), 'Linearized theory of peridynamic states', J. Elasticity 99, 85-111.

S. Silling, M. Epton, O. Weckner, J. Xu and E. Askari (2007), 'Peridynamic states and constitutive modeling', J. Elasticity 88, 151-184.

L. Silvestre (2017), 'Regularity of the obstacle problem for a fractional power of the Laplace operator', Comm. Pure Appl. Math. 60, 67-112.

R. Slevinsky (2019), 'Fast and backward stable transforms between spherical harmonic expansions and bivariate Fourier series', Appl. Comput. Harmon. Anal. 47, 585-606.

R. Slevinsky, H. Montanelli and Q. Du (2018), 'A spectral method for nonlocal diffusion operators on the sphere', J. Comput. Phys. 372, 893-911.

I. Sokolov, J. Klafter and A. Blumen (2002), 'Fractional kinetics', Physics Today $\mathbf{5 5}, 48-54$.

F. Song and G. Karniadakis (2018), A universal fractional model of wall-turbulence. arXiv: 1808.10276

P. Stinga (2019), User's guide to the fractional Laplacian and the method of semigroups. In Handbook of Fractional Calculus with Applications, Vol. 2: Fractional Differential Equations (A. Kochubei and Y. Luchko, eds), De Gruyter, pp. $235-265$.

P. Stinga and J. Torrea (2010), 'Extension problem and Harnack's inequality for some fractional operators', Comm. Partial Differential Equations 35, 20922122.

H.-G. Sun, X. Liu, Y. Zhang, G. Pang and R. Garrard (2017), 'A fast semi-discrete Kansa method to solve the two-dimensional spatiotemporal fractional diffusion equation', J. Comput. Phys. 345, 74-90.

B. Swaford (2001), 'Turbulent relative dispersion', Ann. Rev. Fluid Mech. 33, 289317.

C. Tadjeran, M. Meerschaert and H.-P. Scheffler (2006), 'A second-order accurate numerical approximation for the fractional diffusion equation', J. Comput. Phys. 213, 205-213.

E. Tadmor (2007), Filters, mollifiers and the computation of the Gibbs phenomenon. In Acta Numerica, Vol. 16, Cambridge University Press, pp. 305378.

T. Tang, L.-L. Wang, H. Yuan and T. Zhou (2019), Rational spectral methods for PDEs involving fractional Laplacian in unbounded domains. arXiv:1905.02476

T. Tang, H. Yuan and T. Zhou (2018), 'Hermite spectral collocation methods for fractional PDEs in unbounded domains', Commun. Comput. Phys. 24, 11431168.

Y. Tao, X. Tian and Q. Du (2017), 'Nonlocal diffusion and peridynamic models with Neumann type constraints and their numerical approximations', Appl. Math. Comput. 305, 282-298.

Y. Tao, X. Tian and Q. Du (2019), 'Nonlocal models with heterogeneous localization and their application to seamless local-nonlocal coupling', Multiscale Model. Simul. 17, 1052-1075.

M. Taylor (1996), Partial Differential Equations I, Springer. 
H. Tian, L. Ju and Q. Du (2015), 'Nonlocal convection-diffusion problems and finite element approximations', Comput. Methods Appl. Mech. Engrg 289, 60-78.

H. Tian, L. Ju and Q. Du (2017), 'A conservative nonlocal convection-diffusion model and asymptotically compatible finite difference discretization', Comput. Methods Appl. Mech. Engrg 320, 46-67.

X. Tian (2017), Nonlocal models with a finite range of nonlocal interactions. PhD thesis, Columbia University.

X. Tian and Q. Du (2013), 'Analysis and comparison of different approximations to nonlocal diffusion and linear peridynamic equations', SIAM J. Numer. Anal. 51, 3458-3482.

X. Tian and Q. Du (2014), 'Asymptotically compatible schemes and applications to robust discretization of nonlocal models', SIAM J. Numer. Anal. 52, 16411665 .

X. Tian and Q. Du (2015), 'Nonconforming discontinuous Galerkin methods for nonlocal variational problems', SIAM J. Numer. Anal. 53, 762-781.

X. Tian and Q. Du (2017), 'Trace theorems for some nonlocal function spaces with heterogeneous localization', SIAM J. Math. Anal. 49, 1621-1644.

X. Tian and Q. Du (2020), 'Asymptotically compatible schemes for robust discretization of parametrized problems with applications to nonlocal models', SIAM Rev. 62, 199-227.

X. Tian and B. Engquist (2019), 'Fast algorithm for computing nonlocal operators with finite interaction distance', Commun. Math. Sci. 17, 1653-1670.

X. Tian, Q. Du and M. Gunzburger (2016), 'Asymptotically compatible schemes for the approximation of fractional Laplacian and related nonlocal diffusion problems on bounded domains', Adv. Comput. Math. 42, 1363-1380.

N. Trask, H. You, Y. Yu and M. Parks (2019), 'An asymptotically compatible meshfree quadrature rule for nonlocal problems with applications to peridynamics', Comput. Methods Appl. Mech. Engrg 343, 151-165.

E. Valdinoci (2009), 'From the long jump random walk to the fractional Laplacian', Bol. Soc. Esp. Mat. Apl. SeMA 49, 33-44.

C. Vollmann and V. Schulz (2019), 'Exploiting multilevel Toeplitz structures in high dimensional nonlocal diffusion', Comput. Visual. Sci. 20, 2946.

H. Wang and T. S. Basu (2012), 'A fast finite difference method for two-dimensional space-fractional diffusion equations', SIAM J. Sci. Comput. 34, A2444A2458.

H. Wang and N. Du (2013), 'A fast finite difference method for three-dimensional time-dependent space-fractional diffusion equations and its efficient implementation', J. Comput. Phys. 253, 50-63.

H. Wang and N. Du (2014), 'Fast alternating-direction finite difference methods for three-dimensional space-fractional diffusion equations', J. Comput. Phys. 258, 305-318.

H. Wang and H. Tian (2012), 'A fast Galerkin method with efficient matrix assembly and storage for a peridynamic model', J. Comput. Phys. 231, 77307738 .

H. Wendland (1995), 'Piecewise polynomial, positive definite and compactly supported radial functions of minimal degree', Adv. Comput. Math. 4, 389-396. 
H. Wendland (2005), Scattered Data Approximation, Vol. 17 of Cambridge Monographs on Applied and Computational Mathematics, Cambridge University Press.

D. Witman, M. Gunzburger and J. Peterson (2017), 'Reduced-order modeling for nonlocal diffusion problems', Int. J. Numer. Meth. Fluids 83, 307-327.

F. Xu, M. Gunzburger and J. Burkardt (2016a), 'A multiscale method for nonlocal mechanics and diffusion and for the approximation of discontinuous functions', Comput. Methods Appl. Mech. Engrg 307, 117-143.

F. Xu, M. Gunzburger, J. Burkardt and Q. Du (2016b), 'A multiscale implementation based on adaptive mesh refinement for the nonlocal peridynamics model in one dimension', Multiscale Model. Simul. 14, 398-429.

K. Xu and E. Darve (2018a), Efficient numerical method for models driven by Lévy process via hierarchical matrices. arXiv:1812.08324

K. Xu and E. Darve (2018b), Spectral method for the fractional Laplacian in 2D and 3D. arXiv:1812.08325

C. Yan, W. Cai and X. Zeng (2013), 'A parallel method for solving Laplace equations with Dirichlet data using local boundary integral equations and random walks', SIAM J. Sci. Comput. 35, B868-B889.

H. You, Y. Yu and D. Kamensky (2019), An asymptotically compatible formulation for local-to-nonlocal coupling problems without overlapping regions. arXiv: 1912.06270

M. Zayernouri and G. Karniadakis (2014), 'Exponentially accurate spectral and spectral element methods for fractional ODEs', J. Comput. Phys. 257, 460480.

M. Zayernouri, M. Ainsworth and G. Karniadakis (2015a), 'Tempered fractional Sturm-Liouville eigenproblems', SIAM J. Sci. Comput. 37, A1777-A1800.

M. Zayernouri, M. Ainsworth and G. Karniadakis (2015b), 'A unified PetrovGalerkin spectral method for fractional PDEs', Comput. Methods Appl. Mech. Engrg 283, 1545-1569.

L. Zhang, H.-W. Sun and H.-K. Pang (2015), 'Fast numerical solution for fractional diffusion equations by exponential quadrature rule', J. Comput. Phys. 299, $130-143$.

W. Zhang, J. Yang, J. Zhang and Q. Du (2017), 'Artificial boundary conditions for nonlocal heat equations on unbounded domain', Commun. Comput. Phys. 21, 16-39.

X. Zhang, M. Gunzburger and L. Ju (2016a), 'Nodal-type collocation methods for hypersingular integral equations and nonlocal diffusion problems', Comput. Methods Appl. Mech. Engrg 299, 401-420.

X. Zhang, M. Gunzburger and L. Ju (2016b), 'Quadrature rules for finite element approximations of 1D nonlocal problems', J. Comput. Phys. 310, 213-236.

Z. Zhang (2019), 'Error estimates of spectral Galerkin methods for a linear fractional reaction-diffusion equation', J. Sci. Comput. 78, 1087-1110.

D. Zhou and B. Schölkopf (2005), Regularization on discrete spaces. In Proceedings of the 27th DAGM Symposium, Vol. 3663 of Lecture Notes in Computer Science, Springer, pp. 361-36.

K. Zhou and Q. Du (2010), 'Mathematical and numerical analysis of linear peridynamic models with nonlocal boundary conditions', SIAM J. Numer. Anal. 48, 1759-1780. 